Florida International University FIU Digital Commons

\title{
Theoretical Investigation of Intra- and Inter-cellular Spatiotemporal Calcium Patterns in Microcirculation
}

Jaimit B. Parikh

jpari004@fiu.edu

DOI: $10.25148 /$ etd.FI15032102

Follow this and additional works at: https://digitalcommons.fiu.edu/etd

Part of the Biochemical and Biomolecular Engineering Commons, Biomechanics and

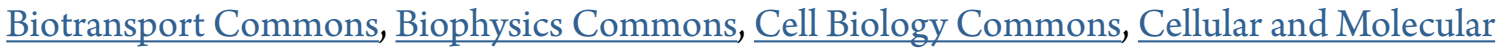
Physiology Commons, Molecular, Cellular, and Tissue Engineering Commons, Non-linear Dynamics Commons, Ordinary Differential Equations and Applied Dynamics Commons, Partial Differential Equations Commons, Systems Biology Commons, and the Transport Phenomena Commons

\section{Recommended Citation}

Parikh, Jaimit B., "Theoretical Investigation of Intra- and Inter-cellular Spatiotemporal Calcium Patterns in Microcirculation" (2015). FIU Electronic Theses and Dissertations. 1927.

https://digitalcommons.fiu.edu/etd/1927 


\section{FLORIDA INTERNATIONAL UNIVERSITY}

Miami, Florida

THEORETICAL INVESTIGATION OF INTRA- AND INTER- CELLULAR SPATIOTEMPORAL CALCIUM PATTERNS IN MICROCIRCULATION

A dissertation submitted in partial fulfillment of

the requirements for the degree of

DOCTOR OF PHILOSOPHY

in

BIOMEDICAL ENGINEERING

by

Jaimit Parikh

2015 
To: Dean Amir Mirmiran

College of Engineering and Computing

This dissertation, written by Jaimit Parikh, and entitled Theoretical Investigation of Intraand Inter-cellular Spatiotemporal Calcium Patterns in Microcirculation, having been approved in respect to style and intellectual content, is referred to you for judgment.

We have read this dissertation and recommend that it be approved.

Anthony McGoron

Jessica Ramella-Roman

Malek Adjouadi

Sharan Ramaswamy

Nikolaos Tsoukias, Major Professor

Date of Defense: January 26, 2015

The dissertation of Jaimit Parikh is approved.

Dean Amir Mirmiran

College of Engineering and Computing

Dean Lakshmi N. Reddi

University Graduate School

Florida International University, 2015 


\section{DEDICATION}

Dedicated to my parents Bharat and Minakshi Parikh 


\section{ACKNOWLEDGMENTS}

I would like to express my sincere thanks to my advisor, Dr. Nikolaos Tsoukias for being such a great mentor; he has been a huge part in the accomplishment of this dissertation. I also want to thank Dr. Adam Kapela for his continued guidance throughout my career at Florida International University, and my lab members, Sridevi Nagaraja, Shabnam Namin, Daniel Rodriguez, Jiali Lei, Florencia Goluboff, Tushar Gadkari, and Kumpal Madrasi for making my time fly.

I extend my appreciation to the Biomedical Engineering Department and the department chair, Dr. Ranu Jung, as well as my committee members, Dr. Anthony McGoron, Dr. Jessica Ramella-Roman, Dr. Malek Adjouadi and Dr. Sharan Ramaswamy for the continued support.

I want to acknowledge the University Graduate School at Florida International University for awarding me the Dissertation Year Fellowship (DYF) and Doctoral Evidence Acquisition Fellowship (DEA). It allowed me to completely focus on completing my dissertation and finishing in a timely manner.

I wish to thank my parents, Bharat and Minakshi, and my sisters, Rachana and Kruti Parikh, for their unconditional love and support. I would also like to thank Samantha Dages for being a fantastic friend and for all the wonderful memories, as well as Sasmita Rath, Ashesh Shah, Chintan Kinariwala, Jacky Gangwani, Jigar Seth, Nikita Shah, Rutvi Trivedi, and all others who made my time more enjoyable. Finally, I would like to thank my cousins, Chirayu and Himanshu Shah, for all their support during my initial years in the United States. 


\begin{abstract}
OF THE DISSERTATION
THEORETICAL INVESTIGATION OF INTRA- AND INTER-CELLULAR SPATIOTEMPORAL CALCIUM PATTERNS IN MICROCIRCULATION
\end{abstract}

by

Jaimit Parikh

Florida International University 2015

Miami, Florida

\title{
Professor Nikolaos Tsoukias, Major Professor
}

Microcirculatory vessels are lined by endothelial cells (ECs) which are surrounded by a single or multiple layer of smooth muscle cells (SMCs). Spontaneous and agonist induced spatiotemporal calcium $\left(\mathrm{Ca}^{2+}\right)$ events are generated in ECs and SMCs, and regulated by complex bi-directional signaling between the two layers which ultimately determines the vessel tone. The contractile state of microcirculatory vessels is an important factor in the determination of vascular resistance, blood flow and blood pressure. This dissertation presents theoretical insights into some of the important and currently unresolved phenomena in microvascular tone regulation. Compartmental and continuum models of isolated EC and SMC, coupled EC-SMC and a multi-cellular vessel segment with deterministic and stochastic descriptions of the cellular components were developed, and the intra- and inter-cellular spatiotemporal $\mathrm{Ca}^{2+}$ mobilization was examined.

Coupled EC-SMC model simulations captured the experimentally observed localized subcellular $\mathrm{EC} \mathrm{Ca}^{2+}$ events arising from the opening of EC transient receptor vanilloid 4 (TRPV4) channels and inositol triphosphate receptors $\left(\mathrm{IP}_{3} \mathrm{Rs}\right)$. These localized $\mathrm{EC} \mathrm{Ca}^{2+}$ 
events result in endothelium-derived hyperpolarization (EDH) and Nitric Oxide (NO) production which transmit to the adjacent SMCs to ultimately result in vasodilation. The model examined the effect of heterogeneous distribution of cellular components and channel gating kinetics in determination of the amplitude and spread of the $\mathrm{Ca}^{2+}$ events. The simulations suggested the necessity of co-localization of certain cellular components for modulation of $\mathrm{EDH}$ and $\mathrm{NO}$ responses. Isolated $\mathrm{EC}$ and SMC models captured intracellular $\mathrm{Ca}^{2+}$ wave like activity and predicted the necessity of non-uniform distribution of cellular components for the generation of $\mathrm{Ca}^{2+}$ waves. The simulations also suggested the role of membrane potential dynamics in regulating $\mathrm{Ca}^{2+}$ wave velocity. The multi-cellular vessel segment model examined the underlying mechanisms for the intercellular synchronization of spontaneous oscillatory $\mathrm{Ca}^{2+}$ waves in individual SMC. From local subcellular events to integrated macro-scale behavior at the vessel level, the developed multi-scale models captured basic features of vascular $\mathrm{Ca}^{2+}$ signaling and provide insights for their physiological relevance. The models provide a theoretical framework for assisting investigations on the regulation of vascular tone in health and disease. 


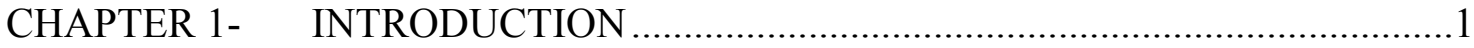

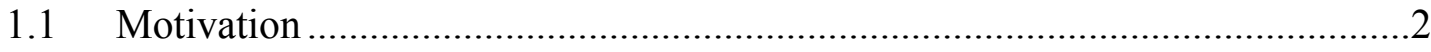

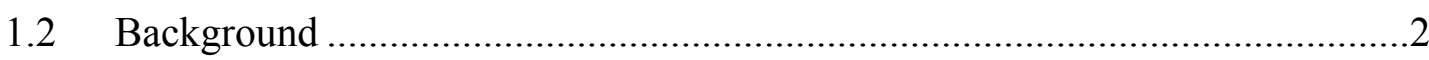

1.2.1 Global $\mathrm{Ca}^{2+}$ events $-\mathrm{Ca}^{2+}$ waves and whole cell oscillations ...................6

1.2.1.1 Physiological role of calcium waves and whole cell oscillations........8

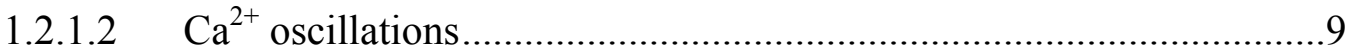

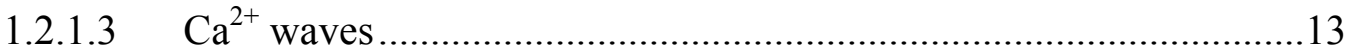

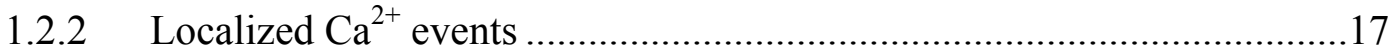

1.2.2.1 $\mathrm{Ca}^{2+}$ sparks (Localized $\mathrm{Ca}^{2+}$ event via RyRs)....................................18

1.2.2.2 $\mathrm{Ca}^{2+}$ puffs (localized $\mathrm{Ca}^{2+}$ events via $\mathrm{IP}_{3} \mathrm{Rs}$ ) ..................................19

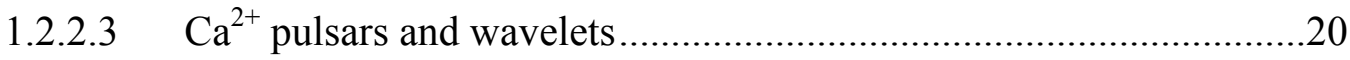

1.2.2.4 $\mathrm{Ca}^{2+}$ sparklets and junctional $\mathrm{Ca}^{2+}$ transients ...................................21

1.2.2.5 Mathematical modeling ..................................................................23

1.2.3 Integrated cytosolic and membrane potential modeling ...........................26

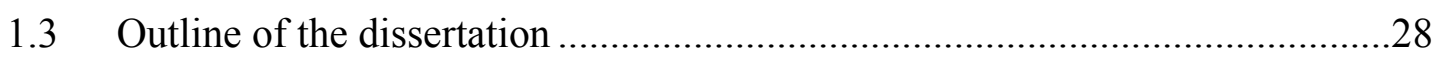

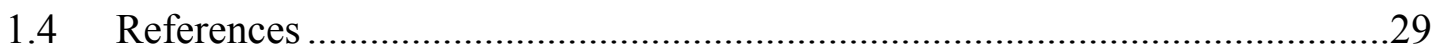

\section{CHAPTER 2- LOCALIZED CALCIUM EVENTS ARISING FROM OPENING}

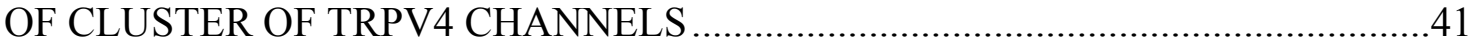

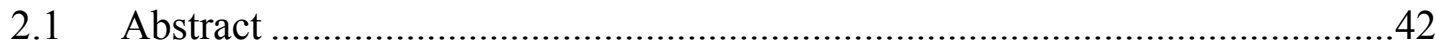

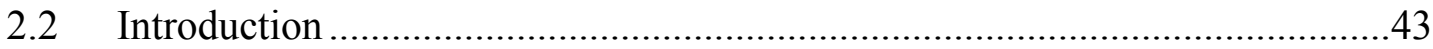

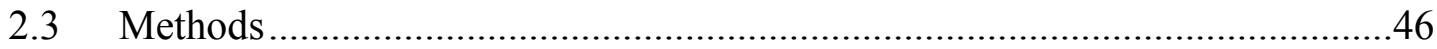

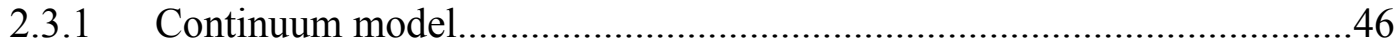

2.3.2 Stochastic opening of TRPV4 channel ………………...........................50

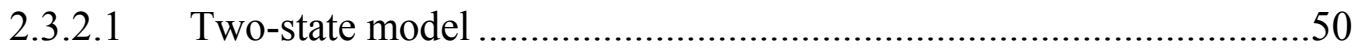

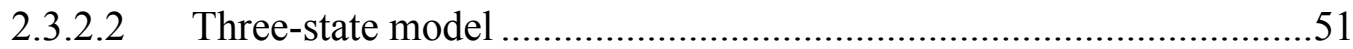

2.3.2.3 Cluster of independent or cooperative TRPV4 channels....................52

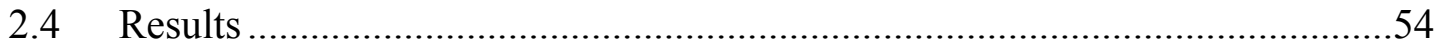

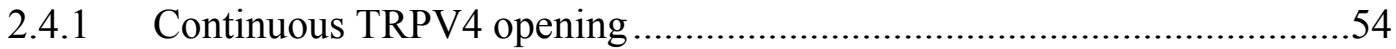

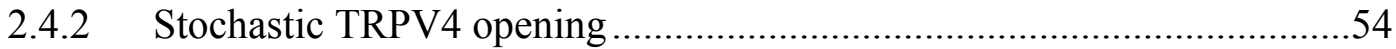

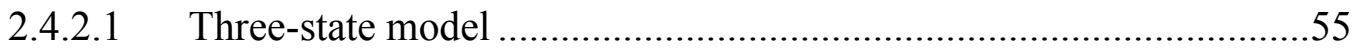

2.4.3 $\mathrm{Ca}^{2+}$ and $\mathrm{V}_{\mathrm{m}}$ dynamics: stochastic continuum model ……………….......57

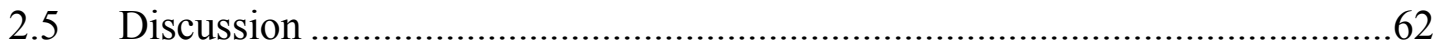




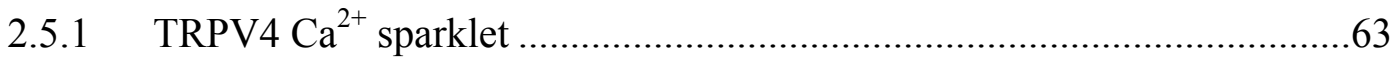

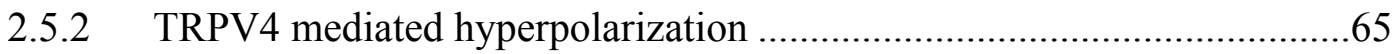

2.5.3 Local versus global $\mathrm{Ca}^{2+}$ activity ......................................................68

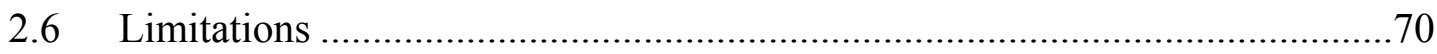

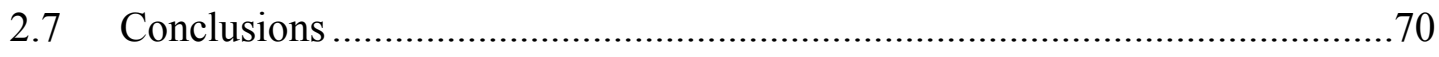

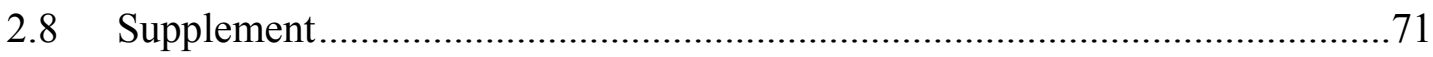

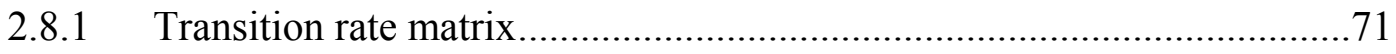

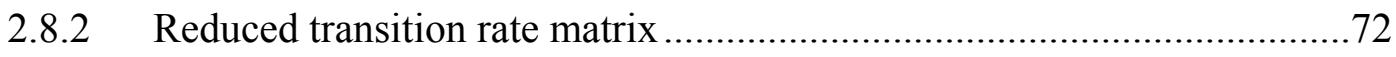

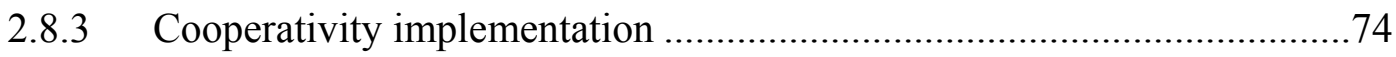

2.8.4 Stochastic TRPV4 opening (Two-state model) .......................................76

2.8.5 Electrical equaivalent of multi-cellular vessel segment............................77

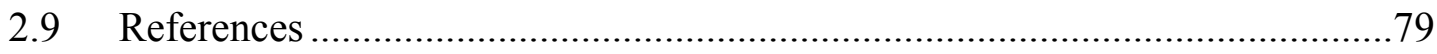

CHAPTER 3- $\quad$ NITRIC OXIDE (NO) MEDIATED VASODILATORY

FEEDBACK RESPONSE AND ITS MODULATION BY ENDOTHELIAL

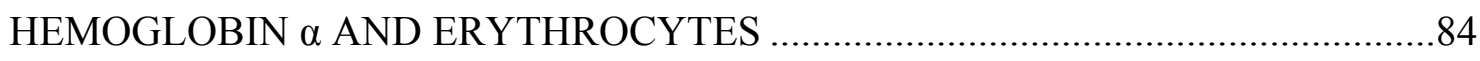

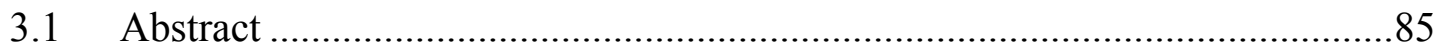

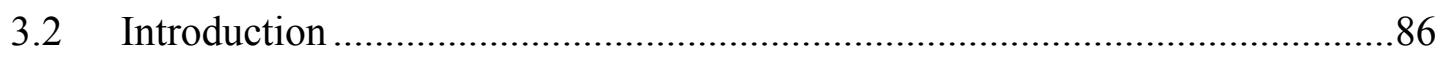

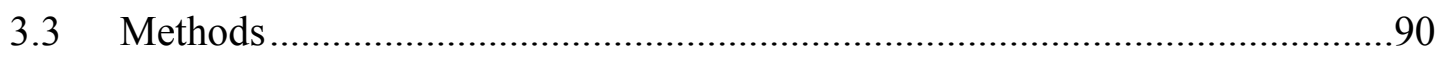

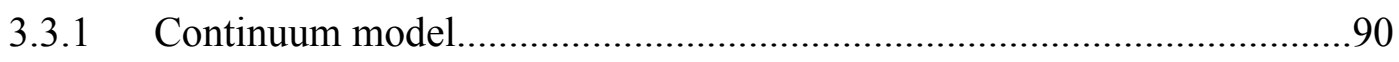

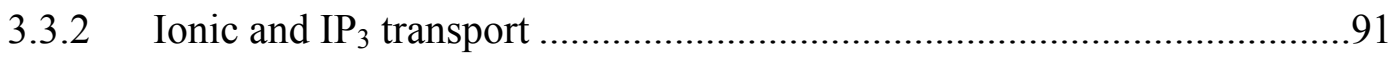

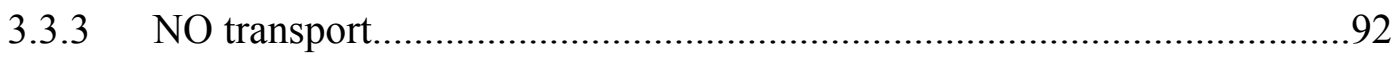

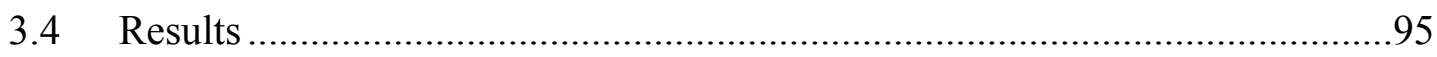

3.4.1 No profiles arising from agonist stimulation of the EC and SMC ............95

3.4.2 eNOS localization during SMC stimulation ............................................99

3.4.3 Hbo localization during SMC stimulation ..............................................100

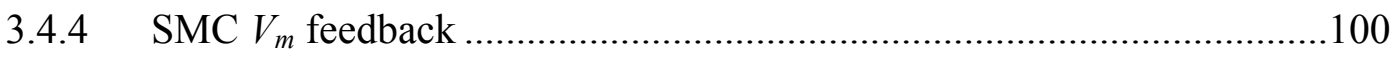

3.4.5 Erythrocyte versus EC Hb $\alpha$ NO consumption ........................................102

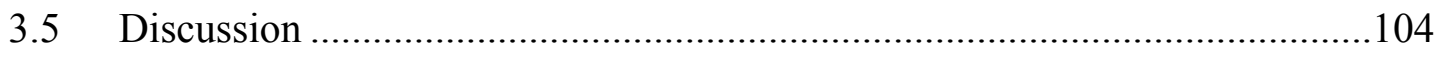

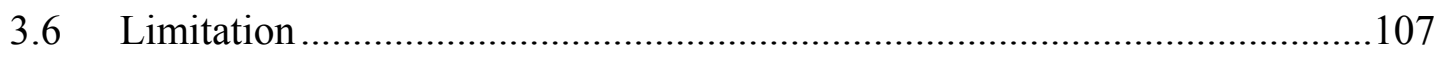

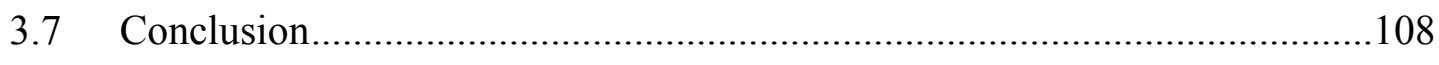

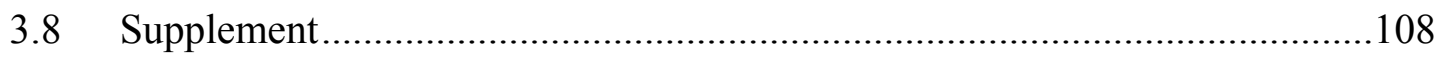

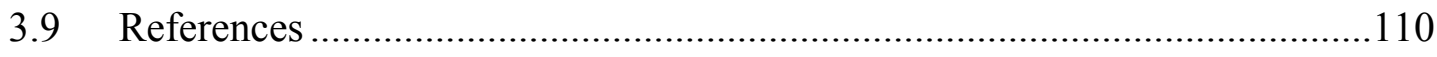

CHAPTER 4- INTRACELLULAR CALCIUM WAVES IN VASCULAR

CELLS 


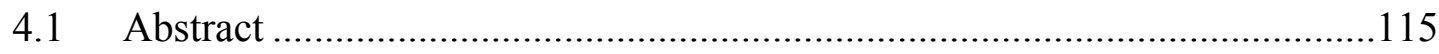

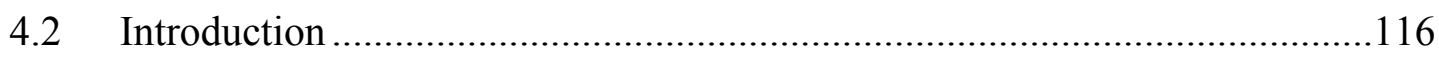

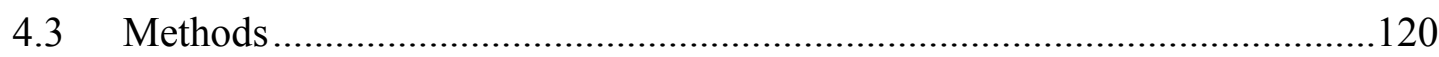

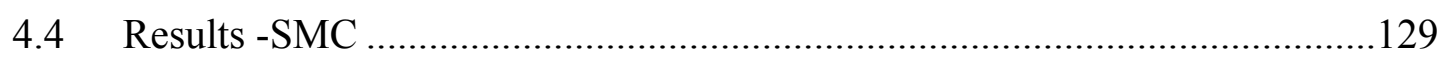

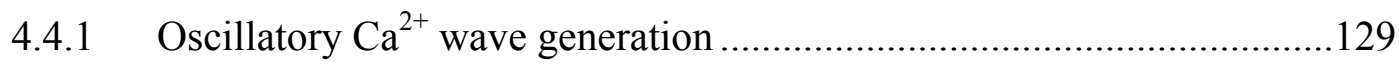

4.4.1.1 Store/Membrane receptor distribution .............................................129

4.4.2 Effect of $V_{\mathrm{m}}$ dynamics on wave velocity .............................................133

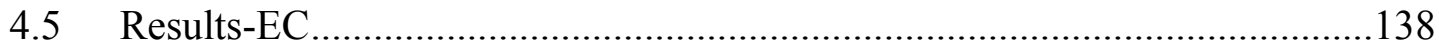

4.5.1 Oscillatory $\mathrm{Ca}^{2+}$ wave generation ....................................................138

4.5.2 Effect of $V_{\mathrm{m}}$ dynamics on wave velocity ................................................141

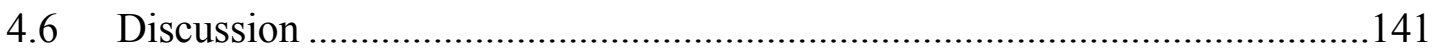

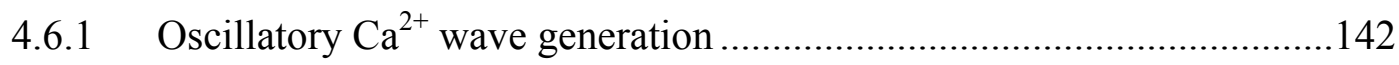

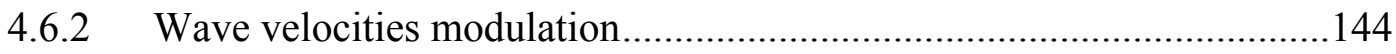

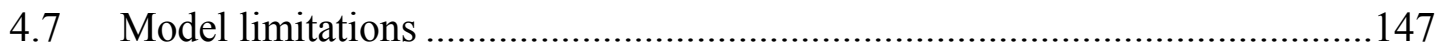

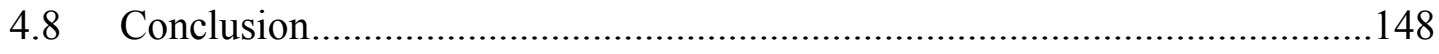

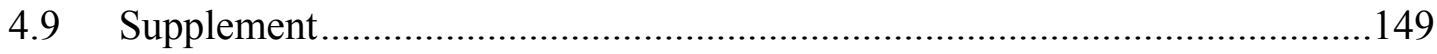

4.9.1 Compartmental model for oscillatory $\mathrm{Ca}^{2+}$ wave ...................................149

4.9.2 Finite element model (FEM) for oscillatory $\mathrm{Ca}^{2+}$ wave .........................150

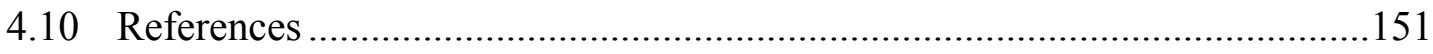

\section{CHAPTER 5- SYNCHRONIZATION OF CALCIUM OSCILLATIONS IN}

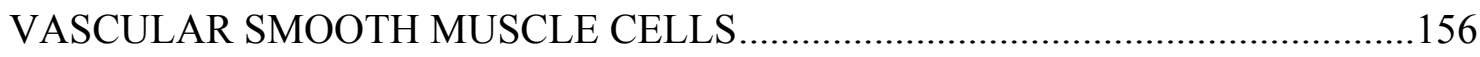

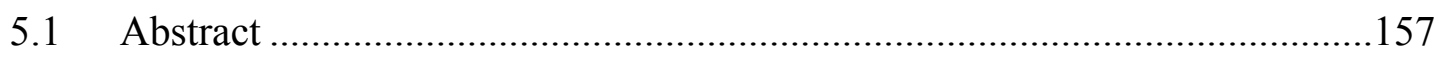

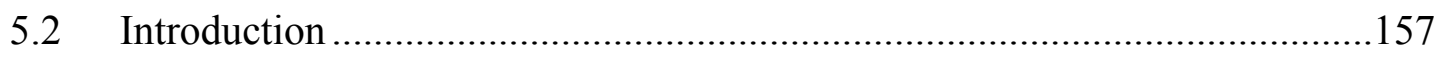

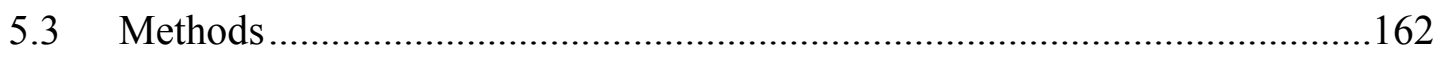

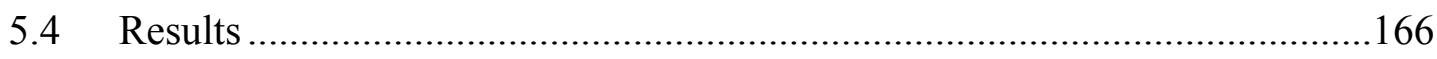

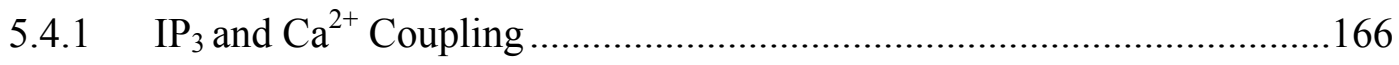

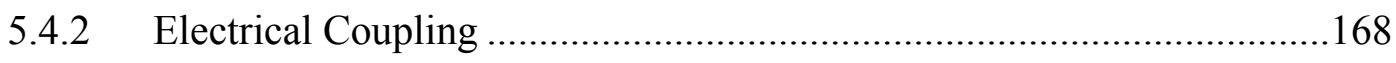

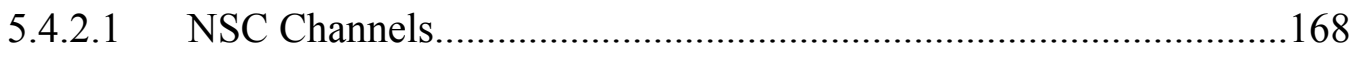

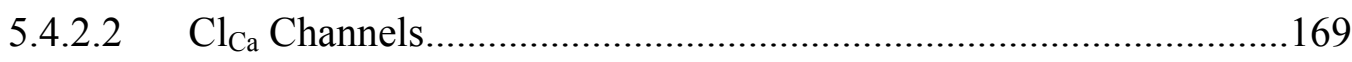

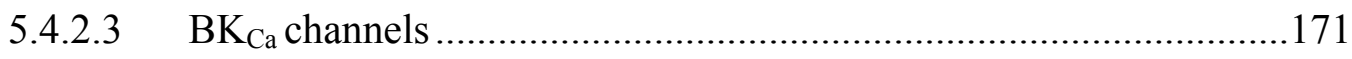

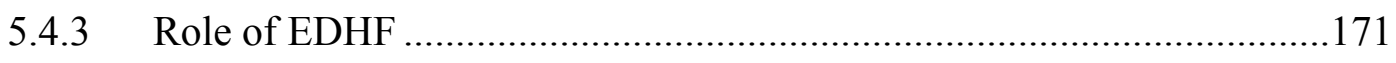

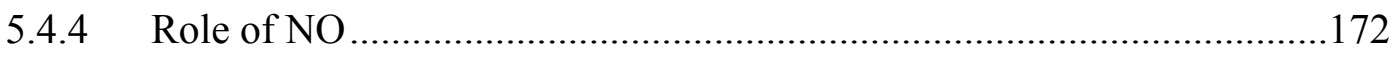

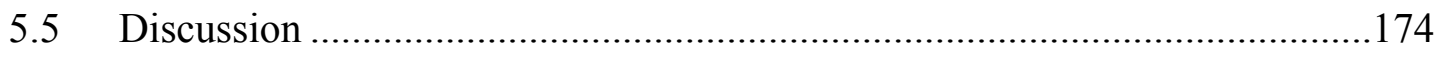




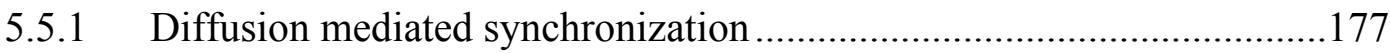

5.5.1.1 Synchronization mediated by $\mathrm{Ca}^{2+}$ diffusion...................................177

5.5.1.2 Synchronization mediated by $\mathrm{IP}_{3}$ diffusion .....................................178

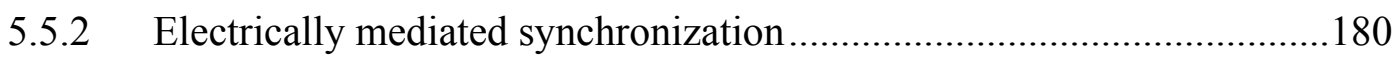

5.5.2.1 Synchronization mediated by electrical coupling............................180

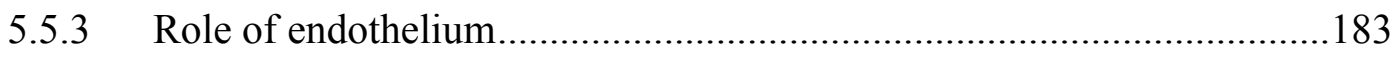

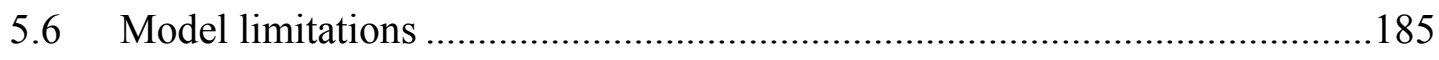

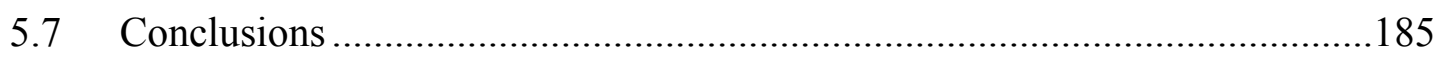

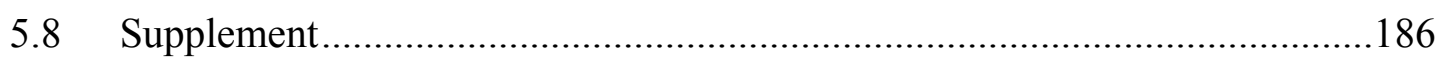

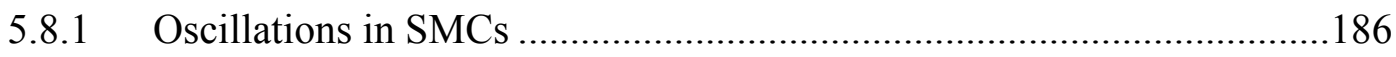

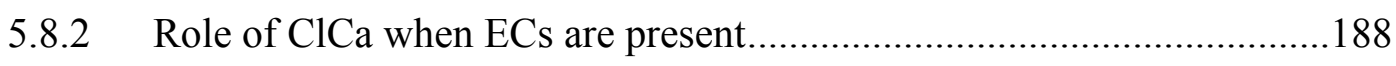

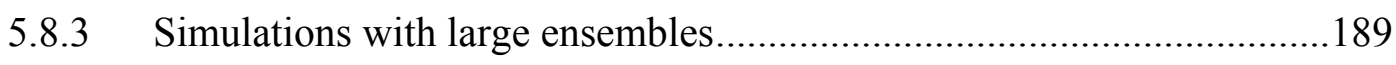

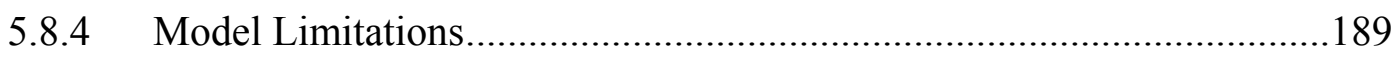

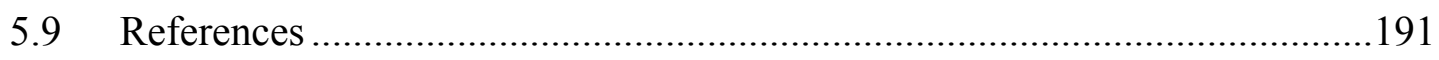

CHAPTER 6- SUMMARY OF THE DISSERTATION ………………………........196

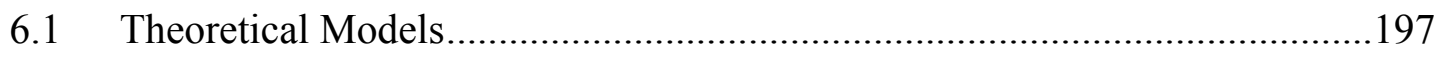

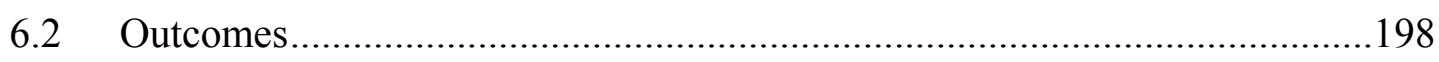

6.2.1 EC TRPV4 mediated subcellular $\mathrm{Ca}^{2+}$ increase....................................198

6.2.2 NO feedback response ....................................................................199

6.2.3 Oscillatory intracellular $\mathrm{Ca}^{2+}$ waves in EC and SMC ............................200

6.2.4 $\mathrm{Ca}^{2+}$ synchronization in arterial vasomotion.........................................20

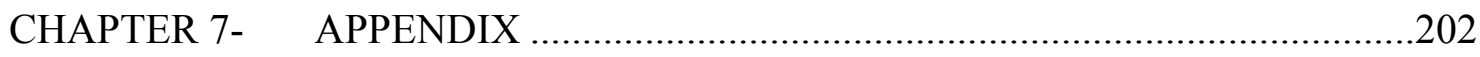

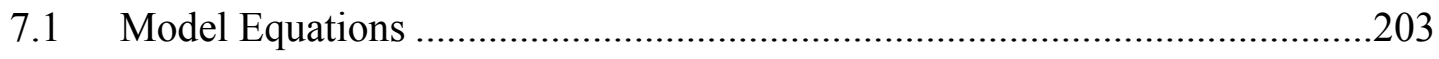

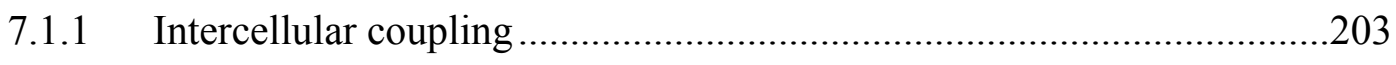

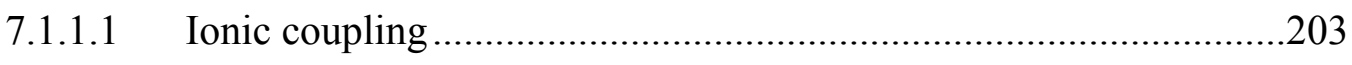

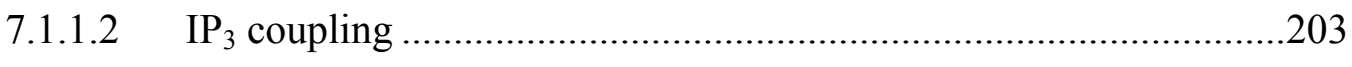

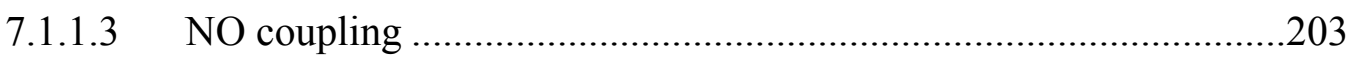

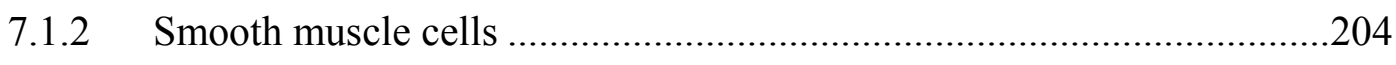

7.1.2.1 Membrane electrophysiology .......................................................204

7.1.2.1.5 Nonselective cation channels.......................................................205

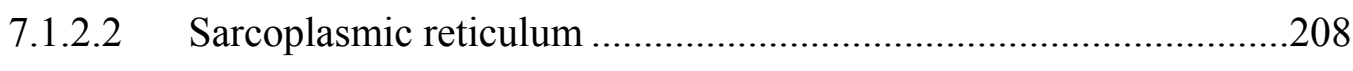

7.1.2.3 $\alpha_{1}$-adrenoceptor activation and $\mathrm{IP}_{3}$ and DAG formation .................209

7.1.2.4 sGC activation and cGMP formation .............................................210 


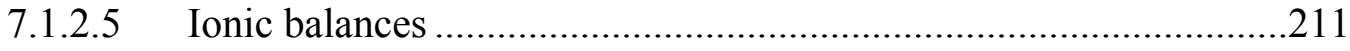

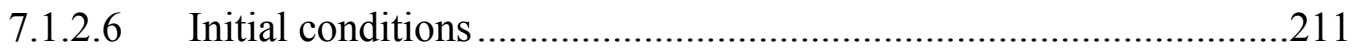

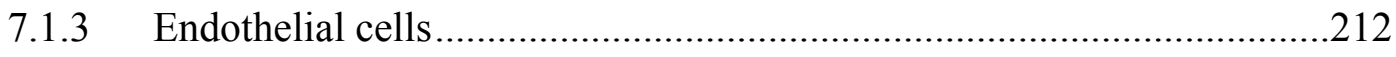

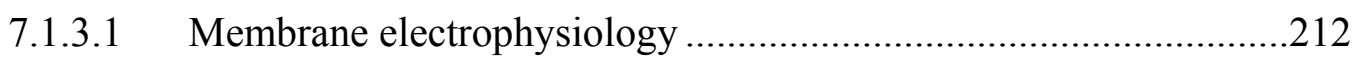

7.1.3.1.6 Nonselective cation channel ......................................................214

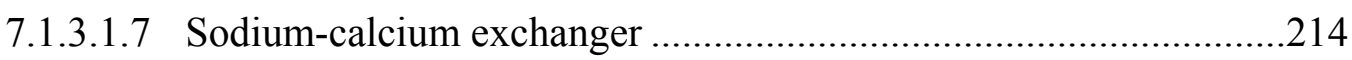

7.1.3.1.9 Sodium-potassium-chloride $\left(\mathrm{Na}^{+} / \mathrm{K}^{+} / 2 \mathrm{Cl}^{-}\right)$cotransport...................215

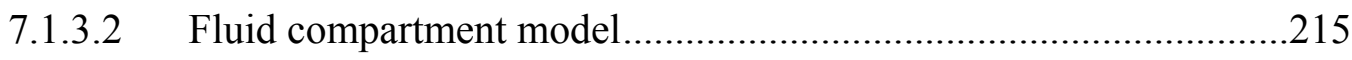

7.1.3.3 Intracellular ionic and material balances ........................................216

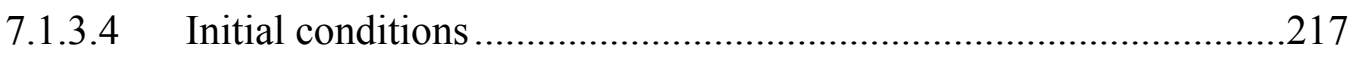

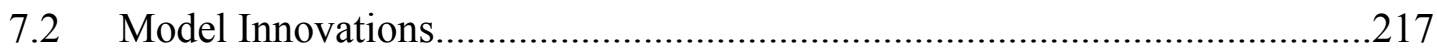

7.3 Model Validation and Sensitivity Analysis...................................................218

VITA 


\section{LIST OF FIGURES}

FIGURE

PAGE

Figure 1.1 Illustration of major endothelial mediated vasodilatory signaling pathways

Figure 1.2 Schematic of localized $\mathrm{Ca}^{2+}$ events reported in the ECs and SMCs 4

Figure 1.3 Schematic of regenerative calcium release and propagating waves 7

Figure 1.4 Illustration for of whole cell oscillation generation 8

Figure 1.5 Cartoon illustration of coupled EC-SMC model with MP 25

Figure 2.1 Schematic of the continuum EC-SMC model, (A) 2D axisymmetric model geometry with SMC and EC as rectangular domains coupled with EC MP and MEGJs (B) Cartoon illustration describing all the channels and pumps incorporated in the EC-MP-SMC continuum model in (A).

Figure 2.2 Cooperativity implementation of four TRPV4 channels, (A) Four TRPV4 channel in a cluster described using a simple two-state (open and close) Markov chain model. The rate parameter $\beta$, for transition of a channel from the closed state to the open state, was increased in the presence of at least one other channel in the open state. (B) TRPV4 channels implemented using a three-state (shut, block and open) Markov chain to capture burst opening of the channel. The rate parameter $\mathrm{k}_{4}$ describing the transition of a channel from the shut state to the open state was increased in the presence of at least one other channel in the open state.

Figure 2.3 Spatial $\mathrm{Ca}^{2+}$ profiles resulting from a single TRPV4 channel opening. (A) Continuous opening of the TRPV4 channel for open times of 10, 100, 200 and 4000 ms resulted in $\mu \mathrm{M} \mathrm{Ca}^{2+}$ concentrations progressively spreading over a larger area. (B) Contour lines $\left(\mathrm{Ca}^{2+}\right.$ concentration equal to $\mathrm{EC}_{50}$ of $\left.\mathrm{IK}_{\mathrm{Ca}}\right)$ indicate increasing $\mathrm{Ca}^{2+}$ spatial spread for 10,100,200 and $4000 \mathrm{~ms}$ of continuous opening of a TRPV4 channel and highlight the time evolution of the cell regions with at least $50 \% \mathrm{IK}_{\mathrm{Ca}}$ activity

Figure 2.4 Stochastic opening of a cluster of four TRPV4 channels implemented using a three-state Markov chain to simulate burst opening of the channel, (A) Illustrative example of a temporal profile of a single TRPV4 channel transition between the conducting (open) and non-conducting states (shut, block). (B) Example of a superposition temporal profile of the TRPV4 cluster, with the level number describing the number of open channels at a given time. Insert shows zoomed in view of a segment of the total simulation time for better visualization.(C) Experiment (Sonkusare et al. 2012) (black solid bar) and cooperative channel gating in the Markov model (black checkered) demonstrated increased open probabilities of 
second, third, and fourth channel openings respectively relative to the binomial distribution (grey solid) and a Markov model (grey checkered) considering independent channels.

Figure 2.5 Representative example of observed temporal $\mathrm{EC} \mathrm{Ca}^{2+}$ and $\mathrm{EC} \mathrm{V}_{\mathrm{m}}$ profiles from the stochastic opening of the TRPV4 cluster in the continuum model, (A) Superposition temporal profile of TRPV4 cluster with cooperative gating kinetics implemented using the three-state model. Insert on right shows zoomed in view for better visualization. (B) $\mathrm{EC} \mathrm{Ca}^{2+}$ concentration around the TRPV4 cluster $\left(0.25 \mu \mathrm{m}^{2}\right.$ area) arising from the TRPV4 openings in (A). (C) $\mathrm{EC} \mathrm{V}_{\mathrm{m}}$ transients follow the $\mathrm{EC} \mathrm{Ca}^{2+}$ events. (D) Local $\mathrm{EC} \mathrm{Ca}{ }^{2+}$ concentration around the TRPV4 cluster $\left(0.25 \mu \mathrm{m}^{2}\right.$ area) observed in different stochastic simulations.

Figure 2.6 Increase in distance of $\mathrm{IK}_{\mathrm{Ca}}$ channel activation arising from burst and cooperative gating kinetics in the TRPV4 cluster, (A) $\mathrm{Ca}^{2+}$ concentration profile in the $\mathrm{EC}$ and the SMC at the time of maximum $\mathrm{Ca}^{2+}$ spread. TRPV4 channel cluster implemented with a two-state model (top: no bursting or cooperative gating kinetics), three-state model (middle: bursting activity) and three-state model with channel interactions (bottom: bursting and cooperativity). Contour lines indicate the $\mathrm{Ca}^{2+}$ concentration equivalent to $\mathrm{EC}_{50}$ of $\mathrm{IK}_{\mathrm{Ca}}$ channels. (B) Radial distance for halfmaximum $\mathrm{K}_{\mathrm{Ca}}$ channel activation in the simulations in $(\mathrm{A})$.

Figure 2.7 Predicted $\mathrm{V}_{\mathrm{m}}$ hyperpolarization induced by a localized $\mathrm{Ca}^{2+}$ increase through TRPV4 channels ( $\mathrm{Ca}^{2+}$ sparklet). (A) Temporal EC $\mathrm{V}_{\mathrm{m}}$ profile (bottom) indicates an average $\mathrm{EC}$ hyperpolarization of $\sim 6 \mathrm{mV}$ during the bursting activity of the TRPV4 cluster (top) in the single EC- single SMC model. (B) Hyperpolarization of the endothelium as a function of sparklet frequency and $\mathrm{IK}_{\mathrm{Ca}}$ localization, predicted for an intact vessel with EC and SMC layers coupled by MEGJ.

Figure 2.8 Plot of $\mathrm{NP}_{\mathrm{o}}$ obtained with 1-20 fold increase in beta (yellow line) and $\mathrm{k}_{4}$ (red dotted line).

Figure 2.9 Stochastic opening of TRPV4 channels described by a two-state Markov chain. (A) Illustrative example of a temporal profile of a single TRPV4 channel transitioning between the close and the open states. (B) A superposition temporal profile of a cluster of four independent TRPV4 channels. The level number describes the number of open channels at a given time point. (C) Open probabilities of first, second, third and fourth level channel openings in a TRPV4 cluster of four channels. The two-state Markov model considering independent channels (grey checkered bars) matches the binomial distribution of independent events (grey solid bar; Equation. 2.7, $\mathrm{P}_{0}=0.045$ ). Experimental data from (Sonkusare et al. 2012) (black solid bar) show increased probability of second, third, and fourth channel openings relative to the binomial distribution. The two-state model with cooperative channel gating (black checkered bar; $\beta^{\prime}=6 \beta$ ) matches the experimentally observed open probabilities 
Figure 2.10 Electrical equivalent of a vessel segment with activated $\mathrm{IK}_{\mathrm{Ca}} \cdot \mathrm{r}_{\mathrm{m}}$ is the net membrane resistance of ECs coupled to SMCs and $\mathrm{R}_{\mathrm{m}}$ is the effective resistance of a EC coupled to a SMC, $R_{I K_{C a}}$ is the total resistance of $\mathrm{IK}_{\mathrm{Ca}}$ channels in a single $\mathrm{EC}$ and $r_{I K_{C a}}$ is the net resistance of $\mathrm{IK}_{\mathrm{Ca}}$ channels in the vessel activated by TRPV4 sparklets.

Figure 3.1 Schematic of the EC-SMC model, (A) Cartoon illustration describing all the channels, pumps and pathways considered in the model. (B) 2D axisymmetric model geometry with SMC, EC and RBC layer as rectangular domains. SMC coupled with EC with MP whose shape is imported from electron microscopy image by (Sandow et al. 2009) and MEGJs. Ion channel currents are uniformly distributed along the top and bottom boundaries of each cell. Inset contains zoomed view of the MP with mesh. eNOS, IP ${ }_{3} \mathrm{Rs}, \mathrm{K}_{\mathrm{Ca}}$ and $\mathrm{Hb} \alpha$ channels are localized in the MP. All other channels and pumps are also present in MP membrane, but proportionally to MP volume. Nitric oxide (NO) is produced in the $\mathrm{EC}$ and can react with $\mathrm{Hb} \alpha$ in the $\mathrm{EC}$, diffuse into the lumen, where it reacts with hemoglobin $(\mathrm{Hb})$ of red blood cells (RBCs). It can also diffuse abluminally and react with oxygen or substrates such as soluble guanylate cyclase (sGC) in smooth muscle.

Figure $3.2 \mathrm{Ca}^{2+}$ (continuous lines) and $\mathrm{IP}_{3}$ (dashed line) concentrations in the EC MP (red lines) and EC bulk (green lines) during (A) NE stimulation of SMC and (B).ACh stimulation of EC

Figure 3.3 Illustrative spatial NO profiles during EC stimulation. (A) Uniform eNOS, Hb $\alpha(-)$, (B) Uniform eNOS, Uniform Hb $\alpha(0.5 \mu \mathrm{M})$, (C) Localized eNOS, $\mathrm{Hb} \alpha(-)$, and (D) Localized eNOS, Localized $\mathrm{Hb} \alpha(0.5 \mu \mathrm{M})$.

Figure 3.4 Average SMC NO concentration during EC stimulation in presence of uniform eNOS, and localized eNOS ( $30 \%$ and $100 \%$ of total eNOS) in the MP. Blue bar represent concentration in the absence of $\mathrm{Hb} \alpha$ and red bars are in the presence of $\mathrm{Hb} \alpha(0.5 \mu \mathrm{M})$.

Figure 3.5 Illustrative spatial NO profiles during SMC stimulation. (A) Uniform eNOS, Hb $\alpha(-)$, (B) Localized eNOS, Hb $\alpha$ (-), (C) Localized eNOS, Uniform Hba $(0.5 \mu \mathrm{M})$, and (D) Localized eNOS, Localized $\mathrm{Hb} \alpha(0.5 \mu \mathrm{M})$

Figure 3.6 Average SMC NO concentration observed during SMC stimulation, in the presence of (A) uniform (U) distributed eNOS in the EC or localized (L) eNOS distribution in the MP $30 \%$ (blue bar), $50 \%$ (orange bar) and 100\% (black bar) of total eNOS) and in the absence (-) of $\mathrm{Hb} \alpha$ or presence of $0.5 \mu \mathrm{M} \mathrm{Hb} \alpha$ distributed uniformly (U) or localized (L).in the MP (B) 30\% eNOS localized in the MP and range of $\mathrm{Hb} \alpha(0-1 \mu \mathrm{M})$ localized in the MP

Figure 3.7 SMC hyperpolarization during SMC stimulation in presence of spatially enriched eNOS in the.MP ( $30 \%$ of total eNOS), (A) under control conditions (green 
continuous line), block of $\mathrm{NO}$ signaling (purple dashed dotted line), absence of $\mathrm{Hb} \alpha$ (red dotted line) and block of $\mathrm{K}_{\mathrm{ca}}$ channels and $\mathrm{NO}$ signaling (orange dashed line). (B) Amount of feedback from NO mediated signaling (purple bar) during $\mathrm{K}_{\mathrm{Ca}}$ block, activation of $\mathrm{K}_{\mathrm{Ca}}$ channels during $\mathrm{NO}$ signaling block (green bar) and presence of both $\mathrm{NO}$ and $\mathrm{K}_{\mathrm{Ca}}$ mediated hyperpolarization.

Figure 3.8 Erythrocyte versus EC Hba regulation of mean SMC NO levels, (A) after $200 \mathrm{~s}$ stimulation of the EC, (B) after $200 \mathrm{~s}$ stimulation of the SMC. (C) Percentage change in mean NO levels in the SMC with increase in localized Hba concentration in the MP during the presence of NO scavenging in the RBC layer (blue lines) or absence of NO scavenging in the RBC layer (black lines).

Figure 3.9 Schematic for analytical analysis of the NO consumption by Hba in the $\mathrm{EC}$ and its consumption by the RBCs. NO production $\left(\mathrm{Q}_{\mathrm{NO}}\right)$ is considered in the $\mathrm{EC}$ layer and its diffuses freely towards the lumen where its concentration is considered to be zero due to its rapid scavenging by the RBCs. NO scavenging in the ECs by $\mathrm{Hb} \alpha$ is considered a first order reaction $\left(\mathrm{K}_{\mathrm{Hb}}[\mathrm{Hb} \alpha][\mathrm{NO}]\right)$.

Figure 4.1 (A) Schematic diagram of single cell EC and SMC model divided into compartments (B) Zoomed in view of a single SMC compartment showing all the incorporated components. $\mathrm{BK}_{\mathrm{Ca}}$-large-conductance $\mathrm{Ca}^{2+}$-activated $\mathrm{K}^{+}$channels; $\mathrm{SOC}$ - store-operated channel; $\mathrm{NSC}$ - nonselective cation channel, $\mathrm{CaCC}$ and $\mathrm{Cl}_{\mathrm{Ca}}-$ $\mathrm{Ca}^{2+}$-activated chloride channel; $\mathrm{NaK}-\mathrm{Na}^{+}-\mathrm{K}^{+}$-ATPase; PMCA - plasma membrane $\mathrm{Ca}^{2+}$-ATPase; $\mathrm{NCX}-\mathrm{Na}^{+} / \mathrm{Ca}^{2+}$ exchanger; $\mathrm{NaKCl}-\mathrm{Na}^{+}-\mathrm{K}^{+}-\mathrm{Cl}^{-}$ cotransport; $\mathrm{K}_{\mathrm{v}}$ - voltage-dependent $\mathrm{K}^{+}$channel; $\mathrm{K}_{\text {leak }}$ - unspecified $\mathrm{K}^{+}$leak current; VOCC - voltage-operated $\mathrm{Ca}^{2+}$ channels; $\mathrm{SR}$ - sarcoplasmic reticulum; $\mathrm{IP}_{3} \mathrm{R}-\mathrm{IP}_{3}$ receptor; RyR - ryanodine receptor; SERCA - SR Ca ${ }^{2+}$-ATPase; CSQN calsequestrin; CM - calmodulin; R - receptor; G - G-protein; DAG - diacylglycerol; PLC - phospholipase C; sGC - soluble guanylate cyclase; cGMP - cyclic guanosine monophosphate. (C) Zoomed in view of a single EC compartment revealing all the incorporated components. $\mathrm{K}_{\mathrm{ir}}$ - inward rectifier $\mathrm{K}^{+}$channel; VRAC - volumeregulated anion channel; $\mathrm{SK}_{\mathrm{Ca}}, \mathrm{IK}_{\mathrm{Ca}}$ and $\mathrm{BK}_{\mathrm{Ca}}-$ small-, intermediate-, and largeconductance $\mathrm{Ca}^{2+}$-activated $\mathrm{K}^{+}$channels; $\mathrm{SOC}$ - store-operated channel; NSC nonselective cation channel, $\mathrm{CaCC}$ and $\mathrm{Cl}_{\mathrm{Ca}}-\mathrm{Ca}^{2+}$-activated chloride channel; $\mathrm{NaK}$ $-\mathrm{Na}^{+}-\mathrm{K}^{+}$-ATPase; PMCA - plasma membrane $\mathrm{Ca}^{2+}$-ATPase; $\mathrm{NCX}-\mathrm{Na}^{+} / \mathrm{Ca}^{2+}$ exchanger; $\mathrm{NaKCl}-\mathrm{Na}^{+}-\mathrm{K}^{+}-\mathrm{Cl}^{-}$cotransport; $\mathrm{K}_{\mathrm{V}}-$ voltage-dependent $\mathrm{K}^{+}$channel; ; $\mathrm{K}_{\text {leak }}$ - unspecified $\mathrm{K}^{+}$leak current; ER - endoplasmic reticulum; $\mathrm{IP}_{3} \mathrm{R} 1$ and $\mathrm{IP}_{3} \mathrm{R} 2$ $\mathrm{IP}_{3}$ receptor isoforms.

Figure 4.2 (A) Step variation in adrenergic receptors resulting in generation of oscillatory $\mathrm{Ca}^{2+}$ wave in the SMC. (B) $\mathrm{Ca}^{2+}$ concentration amplitude depicted color coded and plotted with time vertically and distance from left to right (Kymograph) arising from step variation of adrenergic receptor distribution, shown in (A). (C) Kymograph of $\mathrm{Ca}^{2+}$ concentration after $\mathrm{Ca}^{2+}$ diffusion block showing the importance of intracellular $\mathrm{Ca}^{2+}$ signaling for maintenance of oscillatory $\mathrm{Ca}^{2+}$ wave (B). Mean $\mathrm{Ca}^{2+}$ concentrations (D), mean $\mathrm{IP}_{3}$ concentrations (E) and mean $\mathrm{V}_{\mathrm{m}}$ changes $(\mathrm{F})$ 
across the cell length arising from adrenergic receptor distribution under control conditions (green line) and block of inter-compartmental currents (red line). The bars represent the amplitude of oscillations in the individual compartments across the cell.

Figure 4.3 Linearized variation in RyR density in (A) and normalized variation in (B) resulted in SMC oscillatory $\mathrm{Ca}^{2+}$ wave's depicted color coded as a kymograph in (C) and (D) respectively....

Figure 4.4 Increase in oscillatory $\mathrm{Ca}^{2+}$ wave velocity was observed with increased amplitude of forced $\mathrm{V}_{\mathrm{m}}$ oscillations in phase with the average $\mathrm{Ca}^{2+}$ oscillations while decrease in wave velocities with increased amplitude of forced $V_{m}$ oscillations antiphase to the average $\mathrm{Ca}^{2+}$ oscillations (Negative $\mathrm{V}_{\mathrm{m}}$ indicates anti-phase with $\mathrm{Ca}^{2+}$ ). . 134

Figure 4.5 (A) Exponential decrease in oscillatory $\mathrm{Ca}^{2+}$ wave velocity with increased agonist $(\mathrm{NE}=0.29-0.4 \mu \mathrm{M})$ stimulus in SMC model with $\mathrm{NE}$ step variation. (B) Plot of correlation of average $\mathrm{V}_{\mathrm{m}}$ and $\mathrm{Ca}^{2+}$ oscillations against increased $\mathrm{NE}$ concentration. Increasing agonist (NE) stimulus resulted in more out of phase $\left(\mathrm{V}_{\mathrm{m}}{ }^{-}\right.$ $\mathrm{Ca}^{2+}$ oscillations). The bars indicate the range of correlation coefficients observed in the individual compartments of the cell and green diamond indicates the mean value of correlation at particular agonist concentration.

Figure 4.6 In the SMC model with adrenergic receptor step variation (A) clamp of $\mathrm{BK}_{\mathrm{Ca}}$ and NSC channels at different agonist stimulus concentrations $(\mathrm{NE}=0.29-0.4$ $\mu \mathrm{M})$ resulted in significant increase and decrease respectively in oscillatory $\mathrm{V}_{\mathrm{m}}$ amplitude from the control. Error bars indicate variation in Vm (P-P) for varied NE concentrations $(\mathrm{NE}=0.29-0.4 \mu \mathrm{M})(\mathrm{B})$ The variation in oscillatory $\mathrm{V}_{\mathrm{m}}$ amplitude due to NSC clamp were translated to significant increase in wave velocity due to $\mathrm{BK}_{\mathrm{Ca}}$ clamp and significant decrease in wave velocities under NSC clamp. (C) Clamping VOCC channels resulted in significant decrease in wave velocities at lower range of agonist concentrations but no significant effect at higher agonist concentrations whereas $\mathrm{NCX}\left(\mathrm{Na}^{+}-\mathrm{Ca}^{2+}\right.$ exchanger $)$ resulted in increase in oscillatory wave velocities at lower concentration and a very slight decrease at higher agonist concentrations.

Figure 4.7 (A) Step variation in muscarinic receptors resulting in generation of oscillatory $\mathrm{Ca}^{2+}$ wave in the EC. (B) $\mathrm{Ca}^{2+}$ concentration amplitude depicted color coded and plotted with time vertically and distance from left to right (Kymograph) arising from step variation of muscarinic receptor distribution, shown in (A). (C) Kymograph of $\mathrm{Ca}^{2+}$ concentration after $\mathrm{Ca}^{2+}$ diffusion block showing the importance of intracellular $\mathrm{Ca}^{2+}$ signaling for maintenance of oscillatory $\mathrm{Ca}^{2+}$ wave (B). Mean $\mathrm{Ca}^{2+}$ concentrations (D), mean IP 3 concentrations (E) and mean $\mathrm{V}_{\mathrm{m}}$ levels (F) across the cell length arising from muscarinic receptor distribution under control conditions (green line) and block of inter-compartmental currents (red line). The bars represent the amplitude of oscillations in the individual compartments across the cell. 
Figure 4.8 (A) Linear variation in $\mathrm{IP}_{3} \mathrm{R}$ density introduced in the model produced oscillatory $\mathrm{Ca}^{2+}$ waves. (B) Kymograph of $\mathrm{Ca}^{2+}$ concentration from stimulus of EC (0.9 a.u. Ach) with linear distribution of $\mathrm{IP}_{3}$ receptors as shown in (A). (C) Kymographs of $\mathrm{Ca}^{2+}$ concentration after oscillatory $\mathrm{V}_{\mathrm{m}}$ clamp with same stimulatory conditions as in (B) reduced the $\mathrm{Ca}^{2+}$ wave velocity due to change in electrochemical gradient from the $\mathrm{V}_{\mathrm{m}}$ clamp. (D) Kymograph of $\mathrm{Ca}^{2+}$ concentration showing the reduction in frequency of oscillatory $\mathrm{Ca}^{2+}$ waves in the $\mathrm{EC}$ with reduction in strength of agonist stimulus (0.65 a.u. Ach).

Figure 4.9 stimulation of EC cell with $\mathrm{IP}_{3} \mathrm{R}$ gradient resulted in, (A) Significant reduction in wave velocity (red bar) under $V_{m}$ clamp condition from control (yellow bar) during. (B) Increase in wave frequency with increase agonist concentration

Figure 4.10 Cartoon illustration summarizing the mechanism of generation of oscillatory $\mathrm{Ca}^{2+}$ waves and their modulations in (A) SMC, (B) EC. (C) Phase shift in $\mathrm{Ca}^{2+}$ oscillation's in individual compartments arising from modulation $\mathrm{V}_{\mathrm{m}}$ dynamics in the SMC and EC.

Figure 4.11 (A)Agonist (300 nM) induced oscillatory Ca2+ wave in the SMC under step distribution of adrenergic receptors, obtained using discretized compartmental model ( $\mathrm{N}=200$ compartments) Mean steady state $\mathrm{Ca}^{2+}$ profiles(B) and $\mathrm{IP}_{3}$ profile in (C) across the cell in 2-D FEM SMC model under agonist (300 nM). Bars indicated the amplitude of oscillations

Figure 5.1 Schematic diagram of the ECs and SMCs coupled by nonselective homocellular and heterocellular gap junctions permeable to $\mathrm{Ca}^{2+}, \mathrm{Na}^{+}, \mathrm{K}^{+}$, and $\mathrm{Cl}^{-}$ ions, and $\mathrm{IP}_{3} . \mathrm{K}_{\mathrm{ir}}$ - inward rectifier $\mathrm{K}^{+}$channel; VRAC - volume-regulated anion channel; $\mathrm{SK}_{\mathrm{Ca}}, \mathrm{IK}_{\mathrm{Ca}}$ and $\mathrm{BK}_{\mathrm{Ca}}-$ small-, intermediate-, and large-conductance $\mathrm{Ca}^{2+}$ activated $\mathrm{K}^{+}$channels; $\mathrm{SOC}$ - store-operated channel; NSC - nonselective cation channel, $\mathrm{CaCC}$ and $\mathrm{Cl}_{\mathrm{Ca}}-\mathrm{Ca}^{2+}$-activated chloride channel; $\mathrm{NaK}-\mathrm{Na}^{+}-\mathrm{K}^{+}$-ATPase; PMCA - plasma membrane $\mathrm{Ca}^{2+}$-ATPase; $\mathrm{NCX}-\mathrm{Na}^{+} / \mathrm{Ca}^{2+}$ exchanger; $\mathrm{NaKCl}-$ $\mathrm{Na}^{+}-\mathrm{K}^{+}-\mathrm{Cl}^{-}$cotransport; $\mathrm{K}_{\mathrm{V}}$ - voltage-dependent $\mathrm{K}^{+}$channel; $\mathrm{K}_{\text {leak }}$ - unspecified $\mathrm{K}^{+}$ leak current; VOCC - voltage-operated $\mathrm{Ca}^{2+}$ channels; SR/ER - sarco/endoplasmic reticulum; $\mathrm{IP}_{3} \mathrm{R}-\mathrm{IP}_{3}$ receptor; $\mathrm{RyR}$ - ryanodine receptor; SERCA - SR/ER $\mathrm{Ca}^{2+}$ ATPase; CSQN - calsequestrin; CM - calmodulin; R - receptor; G - G-protein; DAG - diacylglycerol; PLC - phospholipase C; sGC - soluble guanylate cyclase; cGMP - cyclic guanosine monophosphate.

Figure 5.2 (A) A population of five SMCs and five ECs is stimulated by NE (0.8 $\mu \mathrm{M})$ and Ach (1 a.u.). $\mathrm{Ca}^{2+}$ oscillations in the SMCs are shown. Inhibition of intercellular $\mathrm{IP}_{3}$ diffusion desynchronized $\mathrm{SMC} \mathrm{Ca}{ }^{2+}$ oscillations. A correlation coefficient (solid line) quantifies level of synchronization with total synchronization having a value of 1 . (B) Same conditions as in (A). $\mathrm{V}_{\mathrm{m}}$ (Top) and $\mathrm{Ca}^{2+}$ oscillations in SMCs (Bottom) are shown. Clamping DAG concentration to its mean value leads to desynchronization and demonstrates the importance of NSC channels. The synchronization was restored by clamping $\mathrm{BK}_{\mathrm{Ca}}$ activation with mean cytosolic $\mathrm{Ca}^{2+}$. 
(C) In a population of five SMCs (ECs absent), NE $(0.3 \mu \mathrm{M})$ induced synchronized $\mathrm{V}_{\mathrm{m}}$ (Top) and $\mathrm{Ca}^{2+}$ oscillations (Bottom). Increasing $\mathrm{NE}$ concentration $(0.4 \mu \mathrm{M})$ desynchronized SMCs, while exogeneous cGMP hyperpolarizes and resynchronized $\mathrm{Ca}^{2+}$ oscillations through its action on $\mathrm{Cl}_{\mathrm{Ca}}$ channels. (D) Same conditions as in (C). $\mathrm{Cl}_{\mathrm{Ca}}$ channels are clamped with mean cytosolic $\mathrm{Ca}^{2+}$. Removal of clamping restores synchronization which is lost after blocking intercellular $\mathrm{IP}_{3}$ diffusion between SMCs.

Figure 5.3 (A) Model simulations in five SMCs and five ECs stimulated with NE (1 $\mu \mathrm{M}$ ) and Ach (0.7 a.u.). Inhibition of endothelial $\mathrm{SK}_{\mathrm{Ca}}$ and $\mathrm{IK}_{\mathrm{Ca}}$ channels depolarizes $\mathrm{V}_{\mathrm{m}}$ (Top) and desynchronizes $\mathrm{Ca}^{2+}$ oscillations (Bottom). (B) In a population of five SMCs prestimulated with NE $(1 \mu \mathrm{M})($ ECs absent), exogenous NO $(150 \mathrm{nM})$ reduces mean $\mathrm{SM} \mathrm{Ca}^{2+}$ from non-oscillatory to oscillatory domain and this yields synchronized oscillations. (C) Top: Five ECs and five SMCs are stimulated with Ach $\left(0.6\right.$ a.u.) and NE $(1.8 \mu \mathrm{M})$. $\mathrm{Ca}^{2+}$ oscillations in SMCs are shown. Clamping the endothelium-derived NO to its average concentration reduced $\mathrm{Ca}^{2+}$ oscillations and abolished synchronization. Bottom: Oscillatory components of $\mathrm{EC} \mathrm{Ca}^{2+}$ (solid line) and endothelium-derived NO (dashed line) show phase shift. (D) In the presence of Ach (1 a.u.) and endothelial NO release, inhibition of cGMP production by block of SM sGC reduced amplitude and synchronization of $\mathrm{Ca}^{2+}$ oscillations through depolarization and $\mathrm{BK}_{\mathrm{Ca}}$ channels. The effect of sGC block could be compensated by $\mathrm{NE}$ adjustment from $0.8 \mu \mathrm{M}$ to $0.4 \mu \mathrm{M}$.

Figure 5.4 Schematic diagram of signaling pathways that may be involved in $\mathrm{Ca}^{2+}$ synchronization, as predicted by the model. $\mathrm{Ca}^{2+}$ increase in one SMC (left) can activate directly $\mathrm{Cl}_{\mathrm{Ca}}$ and $\mathrm{BK}_{\mathrm{Ca}}$ channels, and indirectly NSC channels through $\mathrm{Ca}^{2+}$ sensitive PLC. Variations in channels' activity generate $V_{m}$ oscillations, which spread through gap junctions to neighboring SMCs (right) and modulate VOCC channels and RyRs. Oscillatory $\mathrm{IP}_{3}$, generated by $\mathrm{Ca}^{2+}$-dependent PLC, can diffuse to other cells, and may also have a synchronizing effect by acting on $\mathrm{IP}_{3}$ receptors. $\mathrm{Ca}^{2+}$ elevation in one cell may increase $\mathrm{Ca}^{2+}$ in neighboring cells by direct diffusion of $\mathrm{Ca}^{2+}$ through the gap junctions if SMC-to-SMC gap junctions have sufficient permeability.

Figure 5.5 (A) Bifurcation diagrams of $\mathrm{Ca}^{2+}$ versus NE for single SMC model without EC (solid line) and with EC stimulated by Ach (dashed-dotted line). (B) Representative distribution of oscillation periods in a model with 10 oscillatory SMCs before coupling (o), after coupling without synchronization $(\times)$ and with synchronization $\left(^{*}\right)$. (C) Forced $\mathrm{Ca}^{2+}$ oscillations in non-oscillatory SMC induced by $\mathrm{IP}_{3}$ and electrical coupling with an oscillatory SMC. (D) Unsynchronized $\mathrm{Ca}^{2+}$ oscillations induced by $0.3 \mu \mathrm{M}$ NE were synchronized transiently by a short $10 \mathrm{~ms}$ pulse of high extracellular $\mathrm{K}^{+}(35 \mathrm{mM})$ resetting simultaneously all SMC oscillators. 186

Figure 5.6 A population of five SMCs and five ECs is stimulated by NE $(0.8 \mu \mathrm{M})$ and Ach (1 a.u.). $\mathrm{Ca}^{2+}$ oscillations in the SMCs are shown. Clamping $\mathrm{Cl}_{\mathrm{Ca}}$ channels 
did not elicit desynchronization (A), but total block of $\mathrm{Cl}_{\mathrm{Ca}}$ current can lead to loss of

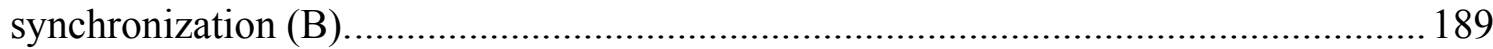

Figure 5.7 Simulations in a population of $80 \mathrm{SMCs}$ and 80 underlying ECs arranged into a cylinder. In the presence of $0.8 \mathrm{NE}$ and 1 a.u. Ach, inhibition of intercellular $\mathrm{IP}_{3}$ diffusion desynchronized the oscillations, similarly to results with five SMCs from Figure 5.2-A.

Figure 5.8 Cluster formation in a population of 80 identical SMCs arranged into a cylinder. When stimulated by $0.4 \mu \mathrm{M} \mathrm{NE}$ from the same initial conditions, the cells produce in-phase oscillations (left). A short local perturbation of the system (10\% NE increase for 1 minute at cell \#1) destabilizes the oscillations and initiates formation of clusters (right). Cells with the same color are in-phase. The curve indicates circular boundary conditions. 190 


\section{ABBREVIATIONS AND ACRONYMS}

EC Endothelial cell

SMC Smooth muscle cell

MEGJ Myoendothelial gap junction

MP Myoendothelial projections

SR Sarcoplasmic reticulum

ER Endoplasmic reticulum

$V_{\mathrm{m}} \quad$ membrane potential

$\mathrm{Ca}^{2+} \quad$ Calcium ion

$\mathrm{K}^{+} \quad$ Potassium

$\mathrm{Na}^{+} \quad$ Sodium

$\mathrm{Cl}^{-} \quad$ Chloride

$\mathrm{IP}_{3} \quad$ Inositol $(1,4,5)$-trisphosphate

CM Calmodulin

CQSN Calsequestrin

MLCP Myosin light-chain phosphatase

MLCK Myosin light chain kinase

PLC Phosopolipase C

DAG Diacylglycerol

PKC Protein kinase $\mathrm{C}$

cGMP Cyclic guanosine monophosphate

$\mathrm{PIP}_{2} \quad$ Phosphatidylinositol-4-5-biphosphate

NO Nitric oxide

$\mathrm{Hb} \quad$ Hemoglobin 


\begin{tabular}{|c|c|}
\hline $\mathrm{Hb} \alpha$ & Hemoglobin $\alpha$ \\
\hline sGC & Soluble guanylate cyclase \\
\hline eNOS & Endothelial nitric oxide synthase \\
\hline $\mathrm{PGI}_{2}$ & Prostacyclin \\
\hline EET & Epoxyeicosatrienoic acids \\
\hline $\mathrm{NE}$ & Norepinephrine \\
\hline Ach & Acetylcholine \\
\hline RyR & Ryanodine receptors \\
\hline $\mathrm{IP}_{3} \mathrm{R}$ & $\mathrm{IP}_{3}$ receptor \\
\hline P2XR & Purinergic $\mathrm{P} 2 \mathrm{X}$ receptor \\
\hline VOCC & Voltage gated calcium channels \\
\hline $\mathrm{SK}_{\mathrm{Ca}}$ & Small conductance calcium activated potassium channel \\
\hline $\mathrm{IK}_{\mathrm{Ca}}$ & Intermediate conductance calcium activated potassium channel \\
\hline $\mathrm{BK}_{\mathrm{Ca}}$ & Large conductance calcium activated potassium channel \\
\hline $\mathrm{NaK}$ & Sodium-potassium ATPase pump \\
\hline $\mathrm{NCX}$ & Sodium-calcium exchanger \\
\hline $\mathrm{PMCa}$ & Plasma membrane $\mathrm{Ca}^{2+}$ ATPase \\
\hline SOC & Store operated $\mathrm{Ca}^{2+}$ channels \\
\hline SERCA & Sarcoplasmic reticulum $\mathrm{Ca}^{2+}$ ATPase \\
\hline NSC & Non-selective cation channels \\
\hline Kir & Inward rectifying potassium channels \\
\hline $\mathrm{K}_{\mathrm{v}}$ & Voltage gated potassium channels \\
\hline VRAC & Volume regulated anion channels \\
\hline $\mathrm{CaCC}$ & Calcium-activated $\mathrm{Cl}^{-}$channels \\
\hline
\end{tabular}




$\begin{array}{ll}\mathrm{Cl}_{\mathrm{Ca}} & \text { Calcium activated } \mathrm{Cl}^{-} \text {channels } \\ \text { TRPV4 } & \text { Transient receptor potential vanilloid channel } 4 \\ \text { TRPM4 } & \text { Transient receptor potential melastatin } 4 \\ \mathrm{R}_{\mathrm{gj}} & \text { Gap junction resistance } \\ \mathrm{CCE} & \text { capacitive calcium entry } \\ \mathrm{EDH} & \text { Endothelium derived hyperpolarization } \\ \mathrm{EDHF} & \text { Endothelium derived hyperpolarizing factor } \\ \mathrm{EDRF} & \text { Endothelium derived relaxing factor } \\ \mathrm{CICR} & \text { Calcium induced calcium release } \\ \mathrm{GHK} & \text { Goldman-Hodgkin-Katz } \\ \text { FEM } & \text { Finite element method } \\ \mathrm{RMA} & \text { Rat mesenteric arteries } \\ \mathrm{CPA} & \text { Cyclopiazonic acid } \\ \mathrm{P}_{0} & \text { Open probability } \\ \mathrm{NP} & \text { Average number of open channels } \\ & \end{array}$


CHAPTER 1-INTRODUCTION 


\subsection{Motivation}

High blood pressure or hypertension affects $33.5 \%$ of US adults (equivalent to around 76.4 million people) and poses a serious health concern. Estimations predict an additional 27 million people (10\% increase) to suffer from hypertension by 2030 and projects a $100 \%$ increase in the medical cost (to reach $\$ 240$ billion) for hypertension (Roger et al. 2012). High blood pressure can lead to increased risk of added cardiovascular diseases including stroke, heart attack and others. Peripheral vascular resistance is increased in virtually all models of hypertension. The total peripheral resistance in turn depends on the arteriolar diameter. Calcium $\left(\mathrm{Ca}^{2+}\right)$ mobilization in microvascular cells plays a central role in the regulation of microcirculatory diameter. Complex mechanisms at the molecular and cellular levels participate in the regulation of vascular diameter. Elucidating the spatiotemporal $\mathrm{Ca}^{2+}$ signaling pathways in the vasculature will provide better understanding of the mechanisms that regulate vascular resistance, blood flow and pressure in health and disease and in future promote better treatments for hypertension.

\subsection{Background}

The increase in intracellular $\mathrm{Ca}^{2+}$ in the smooth muscle cell (SMC) induces contraction of these cells through modulation of MLCK (myosin light-chain kinase) and MLCP (myosin light-chain phosphatase) activities, while in the endothelial cell (EC), $\mathrm{Ca}^{2+}$ increases regulate the release of vasoactive substances. Complex bi-directional myoendothelial interactions via paracrine/gap junction signaling (Yashiro and Duling 2000; Dora et al. 2000; Dora, Doyle, and Duling 1997) modulate the $\mathrm{Ca}^{2+}$ and membrane potential $\left(V_{\mathrm{m}}\right)$ dynamics in both the cells. The myoendothelial interactions often include diffusion of endothelium derived relaxing factors such as nitric oxide (NO) (Palmer, 
Ferrige, and Moncada 1987; Furchgott and Zawadzki 1980; Ignarro et al. 1987), prostacyclin $\left(\mathrm{PGI}_{2}\right)$ (Flower 2006; Vane 1964) and endothelium derived hyperpolarization (EDH) (Chen, Suzuki, and Weston 1988; Garland, Hiley, and Dora 2011) which transmit to adjacent SMCs and result in vessel relaxation (Figure 1.1).

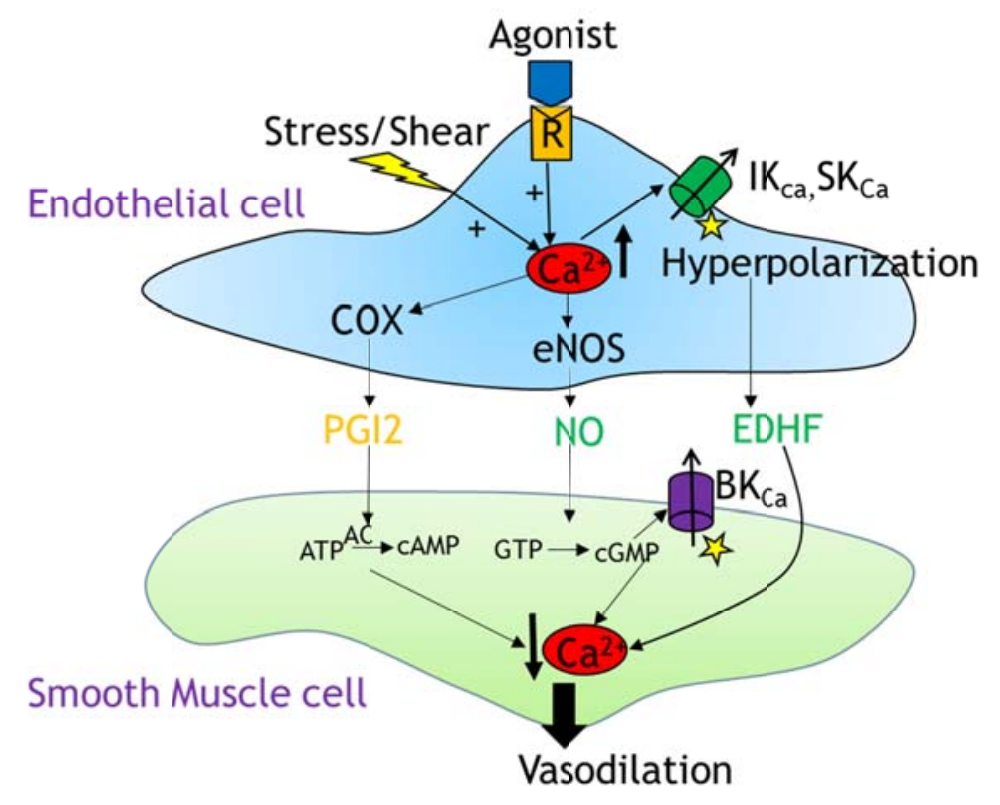

Figure 1.1 Illustration of major endothelial mediated vasodilatory signaling pathways

Since the $\mathrm{Ca}^{2+}$ levels in the vascular cells are governed through complex cellular processes it is expected that these changes are highly organized and occur at various spatial (global or local) and temporal levels (sustained or transient) (Sanders 2001; Berridge 1997). $\mathrm{Ca}^{2+}$ signals range from subcellular events confined to a small region (locally confined events arising from stochastic opening of $\mathrm{Ca}^{2+}$-permeable channels) to whole cell $\mathrm{Ca}^{2+}$ events. Moreover the $\mathrm{Ca}^{2+}$ signals can spread in between the cells to result in inter-cellular $\mathrm{Ca}^{2+}$ mobilization. Figure 1.2 highlights different subcellular localized $\mathrm{Ca}^{2+}$ events observed in the vascular cells mediated through $\mathrm{Ca}^{2+}$-permeable channels (such as voltage gated $\mathrm{Ca}^{2+}$ channels (VOCC), store operated channels (SOC), 
Ryanodine receptor (RyR), inositol 1,4,5-triphosphate receptors $\left(\mathrm{IP}_{3} \mathrm{R}\right)$, Transient receptor potential channel (TRPV4), and Purinergic P2X receptor (P2XR) channels). Earlier reviews on $\mathrm{Ca}^{2+}$ dynamics in a wide variety of cell types can be found in the literature and provide an extensive of this field (Berridge 1997; Berridge, Bootman, and Roderick 2003; Berridge, Lipp, and Bootman 2000; Combettes, Dupont, and Parys 2004; Dupont, Combettes, and Leybaert 2007; Dupont et al. 2000; Falcke 2004; Schuster, Marhl, and Hofer 2002) including some specifically for vascular cells (Amberg and Navedo 2013; Hill-Eubanks et al. 2011; Sanders 2001; Tsoukias 2011; Moccia, Berra-Romani, and Tanzi 2012).

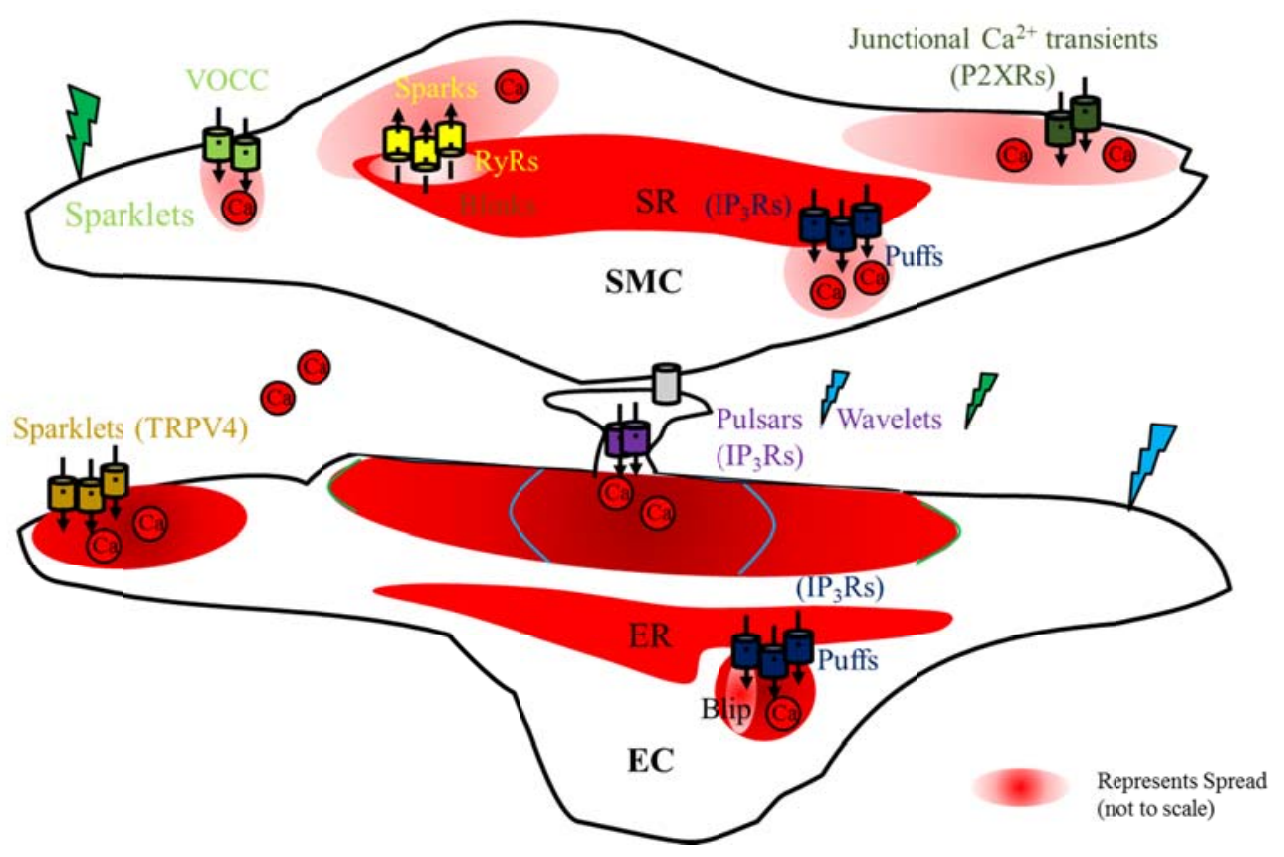

Figure 1.2 Schematic of localized $\mathrm{Ca}^{2+}$ events reported in the ECs and SMCs

Subcellular to whole cell $\mathrm{Ca}^{2+}$ events (i.e. puffs, sparks, sparklets, waves and whole cell events) arising in the microcirculatory vascular ECs and SMCs, and their regulation of the vascular tone are reviewed in this chapter. The characteristics of various $\mathrm{Ca}^{2+}$ events are described in Table 1.1. 
Table 1.1 Characteristics of subcellular to whole cell $\mathrm{Ca}^{2+}$ events

\begin{tabular}{|c|c|c|c|c|c|c|c|}
\hline $\begin{array}{l}\mathrm{Ca}^{2+} \text { Event } \\
\text { Type }\end{array}$ & $\begin{array}{l}\text { Freq } \\
(\mathrm{Hz})\end{array}$ & $\begin{array}{l}\text { Spread } \\
\left(\mu \mathrm{m}^{2}\right)\end{array}$ & $\begin{array}{l}\text { Rise } \\
\text { time } \\
(\mathrm{ms})\end{array}$ & $\begin{array}{l}\text { Decay } \\
\text { time } \\
\left(\mathrm{t}_{1 / 2}\right) \\
(\mathrm{ms})\end{array}$ & Velocities & $\begin{array}{l}\text { Cell } \\
\text { Type }\end{array}$ & REF \\
\hline $\begin{array}{l}\mathrm{Ca}^{2+} \text { sparks } \\
\text { (RyRs) }\end{array}$ & $0.2-1.3$ & 14 & 20 & $50-60$ & NA & SMCs & $\begin{array}{l}\text { (Jaggar et al. } \\
2000)\end{array}$ \\
\hline $\begin{array}{l}\mathrm{Ca}^{2+} \text { puffs } \\
\left(\mathrm{IP} \mathrm{P}_{3} \mathrm{Rs}\right)\end{array}$ & $0.02-0.2$ & $2-4$ & $57-160$ & $107-375$ & NA & $\begin{array}{l}\text { ECs and } \\
\text { SMCs }\end{array}$ & $\begin{array}{l}\text { (Ledoux et al. } \\
\text { 2008; Narayanan, } \\
\text { Adebiyi, and } \\
\text { Jaggar 2012; } \\
\text { Tovey et al. 2001) }\end{array}$ \\
\hline $\begin{array}{l}\mathrm{Ca}^{2+} \text { pulsars } \\
\left(\mathrm{IP}_{3} \mathrm{Rs}\right)\end{array}$ & $0.008-0.1$ & $14-16$ & $\begin{array}{l}163- \\
212\end{array}$ & $146-168$ & NA & ECs & $\begin{array}{l}\text { (Ledoux et al. } \\
\text { 2008) }\end{array}$ \\
\hline $\begin{array}{l}\mathrm{Ca}^{2+} \text { wavelets } \\
\left(\mathrm{IP}_{3} \mathrm{Rs}\right)\end{array}$ & $0.2-0.23$ & $40-51$ & \multicolumn{2}{|c|}{$\begin{array}{l}\text { Longer duration } \\
\text { than pulsars }\end{array}$} & 40 & ECs & (Tran et al. 2012) \\
\hline $\begin{array}{l}\mathrm{Ca}^{2+} \text { sparklet } \\
\text { (VOCC) }\end{array}$ & NA & 0.8 & \multicolumn{2}{|c|}{$\tau_{\text {fast }}=23 \tau_{\text {slow }}=200$} & NA & SMCs & $\begin{array}{l}\text { (Santana et al. } \\
\text { 2008) }\end{array}$ \\
\hline $\begin{array}{l}\mathrm{Ca}^{2+} \text { Sparklet } \\
\text { (TRPV4) }\end{array}$ & $0.02-0.2$ & 11 & \multicolumn{2}{|c|}{$\begin{array}{l}\tau=37 \\
\text { Duration few } \\
\text { seconds }\end{array}$} & NA & ECs & $\begin{array}{l}\text { (Sonkusare et al. } \\
\text { 2012) }\end{array}$ \\
\hline $\begin{array}{l}\text { Junctional } \\
\mathrm{Ca}^{2+} \\
\text { Transient } \\
\text { (P2XR) }\end{array}$ & - & 25 & \multicolumn{2}{|c|}{ Duration: $\mathrm{t}_{1 / 2}=150$} & NA & SMCs & $\begin{array}{l}\text { (Lamont and } \\
\text { Wier 2002) }\end{array}$ \\
\hline $\mathrm{Ca}^{2+}$ waves & $\begin{array}{l}0.06-0.16 \\
(\mathrm{EC}) \\
0.04-1.41 \\
(\mathrm{SMC})\end{array}$ & $8.8-50$ & $\begin{array}{l}500- \\
2000\end{array}$ & $\begin{array}{l}2000- \\
10000\end{array}$ & $1-126$ & $\begin{array}{l}\text { ECs and } \\
\text { SMCs }\end{array}$ & $\begin{array}{l}\text { (Beliveau, } \\
\text { Lapointe, and } \\
\text { Guillemette 2011; } \\
\text { Jaffe 2010; } \\
\text { Narayanan, } \\
\text { Adebiyi, and } \\
\text { Jaggar 2012; } \\
\text { Socha et al. 2012) }\end{array}$ \\
\hline
\end{tabular}

NA denotes not applicable

The modeling approaches in synergy with experimental approaches undertaken for individual $\mathrm{Ca}^{2+}$ event types are also presented. Mathematical modeling offers a systematic approach for the analysis of varied $\mathrm{Ca}^{2+}$ signaling mechanisms, and ranges from minimal models with phenomenological descriptions to models with detailed kinetic descriptions of transmembrane channel currents with deterministic or stochastic implementation. The models also differ in the amount of spatial heterogeneity 
incorporated, with compartmental models incorporating single cytosolic compartment with single or multiple internal stores to continuous models with cytosolic subcompartments, micro-domains and spatial gradients of cellular components. Lastly, a generalized modeling approach to account for cellular heterogeneities and membrane potential dynamics that could reproduce varied multi-cellular $\mathrm{Ca}^{2+}$ events is presented.

\subsubsection{Global $\mathrm{Ca}^{2+}$ events $-\mathrm{Ca}^{2+}$ waves and whole cell oscillations}

Vascular ECs and SMCs exhibit oscillatory $\mathrm{Ca}^{2+}$ behavior arising spontaneously or after agonist stimulation. The underlying mechanisms and cellular components involved in the generation and modulation of the $\mathrm{Ca}^{2+}$ waves and oscillations might be related (Keener and Sneyd 1998), i.e. in both cases the $\mathrm{Ca}^{2+}$ dynamics is mainly associated with $\mathrm{Ca}^{2+}$ release through the store receptors, $\mathrm{Ca}^{2+}$ influx from the membrane channels, and $\mathrm{Ca}^{2+}$ induced $\mathrm{Ca}^{2+}$ release (CICR). $\mathrm{Ca}^{2+}$ released from a cluster of store receptors (RyR, $\mathrm{IP}_{3} \mathrm{R}$ ) can act on itself and diffuse to subsequent adjacent clusters of store receptors to induce regenerative CICR which appears as a propagating $\mathrm{Ca}^{2+}$ wave that traverses across the cell (Figure 1.3).

Cell to cell communication through homo-cellular gap junctions may synchronize oscillatory $\mathrm{Ca}^{2+}$ waves across adjacent vascular cells (Peng et al. 2001; Neylon and Irvine 1990) and induce nearly simultaneous intracellular transient $\mathrm{Ca}^{2+}$ elevation in individual vascular cells referred to as whole cell calcium oscillations. In the SMC, the transition of agonist induced intracellular $\mathrm{Ca}^{2+}$ waves $(0.05 \mathrm{~Hz}$ to $0.1 \mathrm{~Hz})$ to high-frequency $(0.17$ to $0.33 \mathrm{~Hz}$ ) whole cell oscillations has been reported (Peng et al. 2001) and is suggested to initiate the phenomenon of vasomotion. 


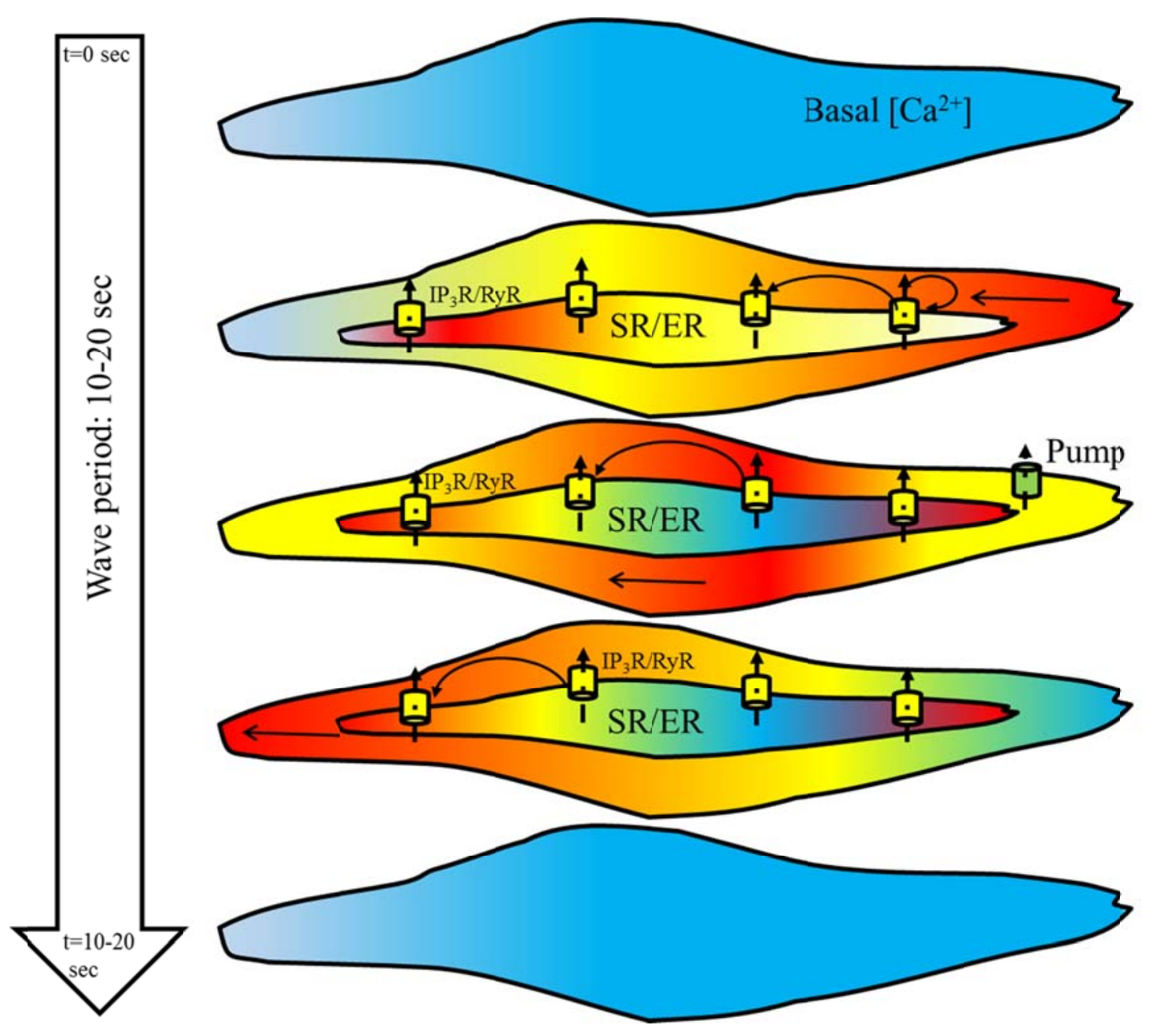

Figure 1.3 Schematic of regenerative calcium release and propagating waves

Under certain stimulatory conditions activation of ionic channels ( such as activation of $\mathrm{Ca}^{2+}$-activated cGMP-dependent chloride channels $\left(\mathrm{Cl}_{\mathrm{Ca}}\right)$ (Matchkov, Aalkjaer, and Nilsson 2004)) can induce depolarizing currents which spreads rapidly across the cell and can result in simultaneous opening of the voltage regulated $\mathrm{Ca}^{2+}$ channels to result in rapid $\mathrm{Ca}^{2+}$ increases throughout the cell and synchronization of localized oscillators to result in whole cell oscillations (Figure 1.4). 


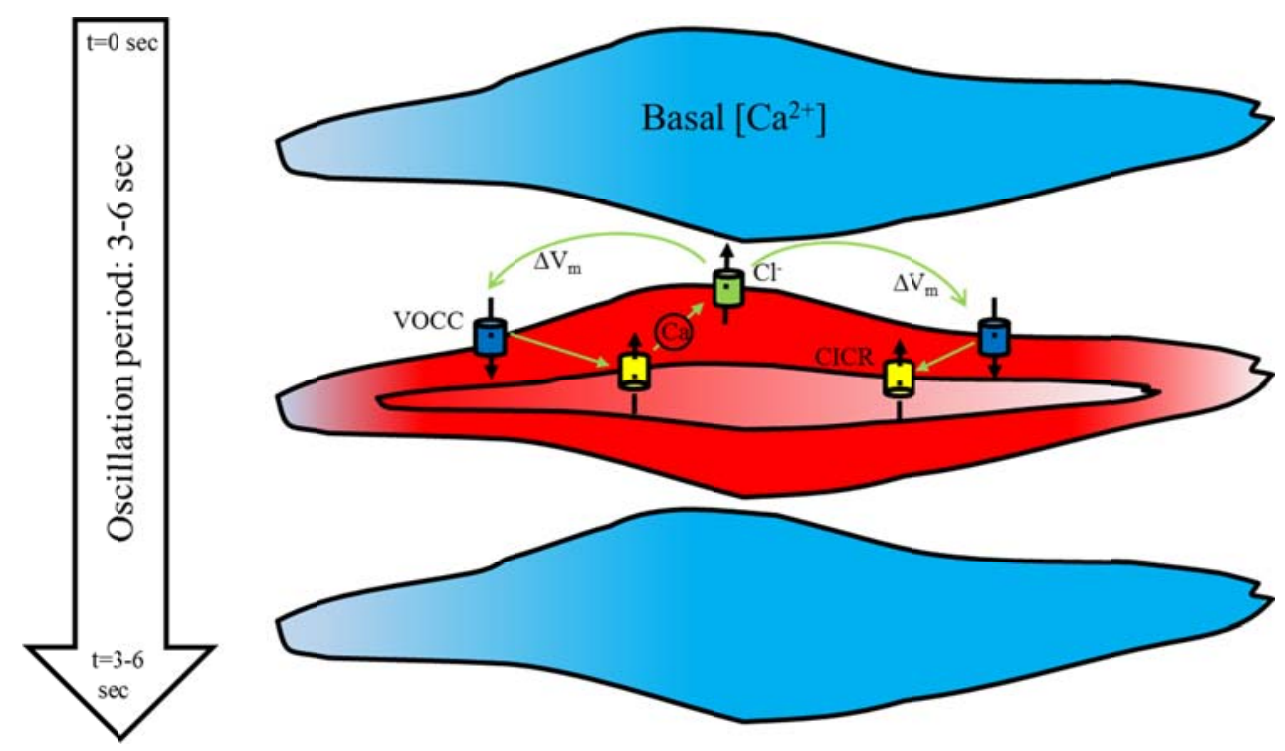

Figure 1.4 Illustration for of whole cell oscillation generation

\subsubsection{Physiological role of calcium waves and whole cell oscillations}

$\mathrm{Ca}^{2+}$ signals can regulate different intracellular responses through varying the frequency of $\mathrm{Ca}^{2+}$ oscillations. For example, the CamKII activity increases exponentially with increase in the frequency of $\mathrm{Ca}^{2+}$ spikes (De Koninck and Schulman 1998). An oscillatory $\mathrm{Ca}^{2+}$ signal than constant steady $\mathrm{Ca}^{2+}$ levels induces higher gene expression in B-lymphocytes (Dupont, Combettes, and Leybaert 2007; Dolmetsch, Xu, and Lewis 1998). In vascular cells $\mathrm{Ca}^{2+}$ waves and oscillations may play a role in the regulation of vessel tone directly through modulation of the contractile apparatus or through regulation of $\mathrm{Ca}^{2+}$ dependent channels like $\mathrm{Ca}^{2+}$ activated chloride channels (Stewart et al. 2012). $\mathrm{Ca}^{2+}$ wave blockade has been shown to result in dilation in cerebral artery SMC (Mufti et al. 2010).Oscillatory $\mathrm{Ca}^{2+}$ waves are suggested to produce significantly less contraction than whole-cell $\mathrm{Ca}^{2+}$ oscillations in airway SMC (Wang et al. 2010). Transient $\mathrm{Ca}^{2+}$ dependent NO production in the EC (Buerk and Riva 1998; Kanai et al. 1995) can be a consequence of $\mathrm{Ca}^{2+}$ waves and oscillations and may improve the efficacy of $\mathrm{NO}$ in 
maintaining vascular tone (Tsoukias, Kavdia, and Popel 2004). Moreover, SMC Ca ${ }^{2+}$ waves may synchronize in neighboring cells to initiate the phenomenon of vasomotion (Peng et al. 2001). On the contrary it has also been suggested that $\mathrm{Ca}^{2+}$ waves have no significant impact on global cytosolic $\mathrm{Ca}^{2+}$ levels and are insignificant to force generation in the myogenic response (Jaggar 2001). The necessity for these two different types of oscillatory $\mathrm{Ca}^{2+}$ pattern (wave-like vs. global transients), their underlying cellular mechanisms and physiological roles need to be better understood.

\subsubsection{2 $\mathrm{Ca}^{2+}$ oscillations}

\subsection{Experiments}

Temporal changes in cytosolic $\mathrm{Ca}^{2+}$ levels under constant agonist stimulations have been observed in the form of repetitive spikes in vascular ECs (Jacob et al. 1988; Socha et al. 2012; Moccia et al. 2003) and SMCs (Jaggar 2001; Lee et al. 2002; Peng et al. 2001; Mauban et al. 2001; Iino, Kasai, and Yamazawa 1994). A regenerative discharge of stored $\mathrm{Ca}^{2+}$ may be responsible for these $\mathrm{Ca}^{2+}$ oscillations (Woods, Cuthbertson, and Cobbold 1986). Although the mechanisms for $\mathrm{Ca}^{2+}$ oscillations in variety of cells have been studied for nearly few decades, ambiguity and controversy still exist on their exact cellular mechanisms. A number of reviews have discussed these issues in detail (Berridge and Galione 1988; Dupont et al. 2011; Dupont, Combettes, and Leybaert 2007; Falcke 2004; Schuster, Marhl, and Hofer 2002). Sequential $\mathrm{Ca}^{2+}$ release from sarcoplasmic/endoplasmic reticulum (SR/ER) via $\mathrm{IP}_{3} \mathrm{Rs}$ (Berridge and Irvine 1989) have been the most studied mechanism for generation of $\mathrm{Ca}^{2+}$ oscillations. $\mathrm{IP}_{3} \mathrm{Rs}$ can generate sustained calcium oscillations thanks to their bell-shaped $\mathrm{Ca}^{2+}$ dependence (Bezprozvanny 1994; Bezprozvanny, Watras, and Ehrlich 1991) i.e., at low $\mathrm{Ca}^{2+}$ levels 
they are activated rapidly and result in $\mathrm{Ca}^{2+}$-induced $\mathrm{Ca}^{2+}$ release (CICR) providing the rising phase of the spike. At higher $\mathrm{Ca}^{2+}$ levels, their slow inhibition results in falling phase of the spike. Constant vs. oscillatory $\mathrm{IP}_{3}$ levels may accompany $\mathrm{Ca}^{2+}$ oscillations, and different isoforms of $\mathrm{IP}_{3} \mathrm{R}$ receptors may play a role in modifying the frequency and sustenance of these oscillations. Both experimental techniques and theoretical models describing the role of $\mathrm{IP}_{3} \mathrm{R}$ in $\mathrm{Ca}^{2+}$ oscillations have been discussed in detail in (Dupont et al. 2011). In addition to $I_{3} R, R y R$ alone (Heppner et al. 2002) or the interplay between $\mathrm{IP}_{3}$ Rs and RyR (Iino, Kasai, and Yamazawa 1994; Boittin et al. 1999; Jaggar and Nelson 2000; Lee et al. 2002) can provide regenerative $\mathrm{Ca}^{2+}$ release. RyR are most extensively characterized in the SMCs, but few studies have suggested their influence on $\mathrm{Ca}^{2+}$ oscillations in some EC types (Jacob et al. 1988; Neylon and Irvine 1990). Mitochondria can also play a role in maintenance of $\mathrm{EC} \mathrm{Ca}^{2+}$ oscillations by modulating cytosolic $\mathrm{Ca}^{2+}$ levels (Ishii, Hirose, and Iino 2006). In addition to oscillations through the store release, $\mathrm{Ca}^{2+}$ oscillations may arise from the $\mathrm{Ca}^{2+}$ influx through the transmembrane channels. In isolated rabbit arteries $\mathrm{Ca}^{2+}$ oscillations through interaction between the largeconductance $\mathrm{Ca}^{2+}$-activated potassium channels $\left(\mathrm{BK}_{\mathrm{Ca}}\right)$ and the VOCC channels have been reported (Omote, Kajimoto, and Mizusawa 1992).

\subsection{Models}

Experimental techniques performed to assess all elements of the regulatory mechanism of $\mathrm{Ca}^{2+}$ oscillations suggest involvement of different channels, pathways and feedback processes that cannot be fully approached by qualitative reasoning. Mathematical models in addition to experimental techniques provide a better tool for understanding the overall $\mathrm{Ca}^{2+}$ dynamics. Many computational models have been 
developed to investigate or simulate oscillations in different cell types, including the EC and SMC. The models differ in the underlying assumption of the type of receptors and channels involved and the oscillatory mechanism, i.e. oscillations through store $\mathrm{IP}_{3} \mathrm{Rs}$ and RyR (cytosolic oscillators) (Koenigsberger et al. 2004; De Young and Keizer 1992; Keizer and Levine 1996), membrane oscillators i.e. oscillations induced through membrane channels (for example voltage gated channels $\mathrm{Ca}^{2+}$ or $\mathrm{K}^{+}$channels (Fioretti, Franciolini, and Catacuzzeno 2005; Zeng, Li, and Chen 2009)) or oscillations arising from periodic variations in metabolic $\mathrm{IP}_{3}$ concentrations (Meyer and Stryer 1988). Moreover they differ in the detail of description ranging from microscopic (stochastic modeling) to macroscopic descriptions (deterministic modeling). The different models have been nicely tabulated in (Dupont et al. 2011). The increase in intra-cellular $\mathrm{Ca}^{2+}$ via store channels in case of cytosolic oscillators or through the membrane channels (membrane oscillators) in the presence of positive feedback (CICR) results in the rising phase of the oscillations in the models. The decline phase of oscillation in these models can be a result of inactivation of $\mathrm{IP}_{3} \mathrm{Rs}$ by high $\left[\mathrm{Ca}^{2+}\right]$ (Bezprozvanny 1994), membrane potential repolarization from slow rundown of $\mathrm{IK}_{\mathrm{Ca}}$ channels (Fioretti, Franciolini, and Catacuzzeno 2005) or termination of RyR-mediated $\mathrm{Ca}^{2+}$ release by store depletion (Kapela, Bezerianos, and Tsoukias 2008). Moreover, theoretical investigation has been carried out to examine the effect of $\mathrm{IP}_{3} \mathrm{R}$ isoforms on cytosolic $\mathrm{Ca}^{2+}$ oscillations through the development of phenomenological models (Dupont and Combettes 2006).

The existing literature for $\mathrm{Ca}^{2+}$ dynamics models in vascular cells (EC and SMC) is reviewed in (Tsoukias 2011). SMCs differ from the electrically non-excitable ECs in their channel and receptor compositions. Although L-type $\mathrm{Ca}^{2+}$ channels and CICR from 
RyR and/or IP ${ }_{3}$-sensitive stores play a predominant role in SMC, the phosphatidylinositol pathway and capacitive calcium entry (CCE) have a significant role in ECs. Furthermore, the type and density of membrane ion channels in the cells exhibit significant differences among different species and tissues and probably change with the size of the vessel even within the same vascular bed. This heterogeneity suggests a need for cell- and vesselspecific detailed models of $\mathrm{Ca}^{2+}$ signaling and dynamics in the vasculature.

A detailed model of $\mathrm{Ca}^{2+}$ dynamics and oscillations in the SMC have been presented in (Kapela, Bezerianos, and Tsoukias 2008). The model predicts spontaneous $\mathrm{Ca}^{2+}$ oscillations generated by repetitive CICR through RyRs and emptying of a small release compartment as release termination mechanism (Kapela, Bezerianos, and Tsoukias 2008). A detailed $\mathrm{EC}$ model that integrates both $\mathrm{EC} \mathrm{Ca}^{2+}$ dynamics and plasmalemma electrical activity to investigate $\mathrm{EC}$ responses to various stimulatory conditions and the relationship between $\mathrm{Ca}^{2+}$ and membrane potential $\left(V_{\mathrm{m}}\right)$ have been presented in (Silva, Kapela, and Tsoukias 2007). However, the model did not predict $\mathrm{Ca}^{2+}$ oscillations.

Majority of the models for intracellular $\mathrm{Ca}^{2+}$ oscillations are deterministic in nature. Recently, Falcke et al. (Falcke 2003) developed stochastic models for $\mathrm{Ca}^{2+}$ oscillations, where oscillations are generated by local stochastic events arising from opening of an $\mathrm{IP}_{3}$ receptor channel. Moreover, Falcke and coworkers have shown that $\mathrm{Ca}^{2+}$ oscillations in some cell types do not obey deterministic dynamics and rather are a sequence of random spikes (Falcke 2003; Skupin et al. 2008). 


\subsubsection{3 $\mathrm{Ca}^{2+}$ waves}

\subsection{Experiments}

Spatially organized $\mathrm{Ca}^{2+}$ waves with diverse propagation velocities have been observed in a variety of cell types (Berridge 1993; Rottingen and Iversen 2000; Berridge and Dupont 1994; Jaffe 2010; Narayanan, Adebiyi, and Jaggar 2012; Huser and Blatter 1997). The observed sarcoplasmic (SR) and endoplasmic reticulum (ER) released $\mathrm{Ca}^{2+}$ waves in vascular cells can be characterized broadly into two distinct types based on the experimental protocol carried out to generate them. In the first type, $\mathrm{Ca}^{2+}$ waves appear from a localized $\mathrm{Ca}^{2+}$ increase via store receptors $\left(\mathrm{IP}_{3} \mathrm{Rs} / \mathrm{RyRs}\right.$ ) (Halidi et al. 2011; Marchant, Callamaras, and Parker 1999; McCarron et al. 2010) acquired experimentally through photolysis of caged $\mathrm{IP}_{3}$ in a small region of the cell (Wang and Augustine 1995). If the localized $\mathrm{Ca}^{2+}$ release is sufficiently strong, it can diffuse across the cell and have amplified response via CICR to result in a propagating $\mathrm{Ca}^{2+}$ wave that traverses the cell. Sensitization of store receptors with presence of basal levels of $\mathrm{Ca}^{2+}$ and/or $\mathrm{IP}_{3}$ might be essential for the propagation via CICR (McCarron et al. 2010). The initiation of such waves usually occurs from the site of the local $\mathrm{IP}_{3}$ release. The second type is characterized as, repetitive or oscillatory $\mathrm{Ca}^{2+}$ waves in the EC and SMC obtained experimentally through uniform agonist stimulus applied throughout the cell. Such waves appear to originate from a fixed site, and might be a result of higher density of membrane or store receptors in that region or microdomain (Olson et al. 2012). Endothelial $\mathrm{Ca}^{2+}$ waves are reported to preferentially originate at specific loci in caveolin-rich cell edges upon uniform stimulation with ATP, bradykinin and thrombin (Isshiki et al. 1998). 
Agonist-induced oscillatory $\mathrm{Ca}^{2+}$ waves have been observed in both the SMCs (Peng et al. 2001; Iino, Kasai, and Yamazawa 1994; Boittin et al. 1999; Jaggar and Nelson 2000; Dai et al. 2007; Dai et al. 2006; Lee et al. 2001; Ruehlmann et al. 2000; Syyong et al. 2009) and ECs (Beliveau, Lapointe, and Guillemette 2011; Huser and Blatter 1997; Isshiki et al. 1998; Socha et al. 2012) of different species. $\mathrm{Ca}^{2+}$ waves can also be classified based on their proposed underlying mechanism. In one scenario, a localized $\mathrm{Ca}^{2+}$ oscillator provides necessary $\mathrm{Ca}^{2+}$ which through diffusion and CICR results in a propagating wave (Keener and Sneyd 1998). In another scenario, the whole cell is oscillatory, and spatial cellular heterogeneity can cause the wave like pattern appearance due to phase differences in oscillatory activity in different region of the cells (Jafri and Keizer 1994). A combination of the two scenarios is also possible. In the later mechanism, the diffusion of $\mathrm{Ca}^{2+}$ or $\mathrm{IP}_{3}$ might not be essential for generation of $\mathrm{Ca}^{2+}$ transients but may provide their phase lock.

Stimulus strength, $\mathrm{Na}^{+}-\mathrm{Ca}^{2+}$ exchanger (NCX), non-selective cation channels (NSCs), VOCC channels, and store receptor channels are suggested to modulate both the frequency and speed of $\mathrm{Ca}^{2+}$ waves and reviewed in (Narayanan, Adebiyi, and Jaggar 2012) The modulation of $\mathrm{Ca}^{2+}$ waves through the plasmalemmal channels and pumps can be a result of altering membrane potential dynamics electrochemical gradient for $\mathrm{Ca}^{2+}$ influx.

\subsection{Models}

Modeling $\mathrm{Ca}^{2+}$ waves requires examination of both spatial and temporal scales and necessitates to account for the description of cytosolic $\mathrm{Ca}^{2+}$ diffusion. Several minimal deterministic reaction-diffusion models of $\mathrm{Ca}^{2+}$ waves, describing $\mathrm{Ca}^{2+}$ and $\mathrm{IP}_{3}$ dynamics 
have been undertaken. Similar to the models of $\mathrm{Ca}^{2+}$ oscillations, the simulations of $\mathrm{Ca}^{2+}$ waves differ in the underlying assumption of the oscillatory mechanism. (For example $\mathrm{Ca}^{2+}$ waves arising from $\mathrm{IP}_{3} \mathrm{R}$ are consider in (Atri et al. 1993; Dupont and Goldbeter 1994; Sneyd et al. 1995), oscillation through combination of $\mathrm{IP}_{3} \mathrm{R}$ and RyR in (Wang et al. 2010)). Moreover, the models differ in type of stimulus i.e. $\mathrm{Ca}^{2+}$ waves generated from localized $\mathrm{Ca}^{2+}$ increase (Atri et al. 1993; Dupont and Goldbeter 1994) which propagates across the cell or under uniform stimulus throughout the cell (Wang et al. 2010) (e.x. through rise of $\mathrm{IP}_{3}$ levels throughout the cell). Non-uniform cellular component distribution (e.x. store receptors) or introduction of noise in the model was essential for spatial heterogeneity for $\mathrm{Ca}^{2+}$ wave generation under uniform stimulus, in the models (Wang et al. 2010; Jacobsen et al. 2007). The initial models were deterministic in nature and provide a good understanding of generation and propagation of these waves. Falcke et al., based on the experimental observations in Xenopus oocytes (Sun et al. 1998) developed stochastic models of $\mathrm{Ca}^{2+}$ waves (Falcke, Tsimring, and Levine 2000; Falcke 2003; Thul and Falcke 2004). They simulated the transition from puffs to waves to fast oscillations with increasing stimulus strength. The waves were a result of nucleation, i.e. opening of at least few clusters of $\mathrm{IP}_{3}$ Rs resulting in localized $\mathrm{Ca}^{2+}$ increase high enough to activate the adjacent clusters inside the cells. Detailed threedimensional model of ventricular myocytes comprising of anisotropic distribution of RyR clusters within a few sarcomeres reconstructed directly from confocal imaging measurements, and simple stochastic CICR release dynamics have shown to produce global $\mathrm{Ca}^{2+}$ wave propagations ( $\mathrm{Li}$ et al. 2010) 
Limited models have examined the role of membrane potential dynamics in regulation of the $\mathrm{Ca}^{2+}$ waves. Jacobsen et al. developed a multi-compartmental vascular SMC model describing both $V_{\mathrm{m}}$ and $\mathrm{Ca}^{2+}$ dynamics (Jacobsen et al. 2007). Modulation in frequency of $\mathrm{Ca}^{2+}$ wave with varying mean $V_{\mathrm{m}}$ was observed in the model. We have recently developed multi-scale model of integrated $\mathrm{Ca}^{2+}-V_{\mathrm{m}}$ dynamics for modeling regulation of vascular tone (Kapela and Tsoukias 2011), appropriate for investigating the role of both cytosolic and membrane components in intracellular $\mathrm{Ca}^{2+}$ waves. The model incorporates diffusion of $\mathrm{Ca}^{2+}, \mathrm{Na}^{+}, \mathrm{K}^{+}, \mathrm{Cl}^{-}$and $\mathrm{IP}_{3}$ and all membrane and store components from our original lumped models of EC and SMC (Kapela, Bezerianos, and Tsoukias 2008; Silva, Kapela, and Tsoukias 2007).

Examination of experimentally observed (Peng et al. 2001) transition of intra-cellular $\mathrm{Ca}^{2+}$ waves to whole-cell oscillations in the SMC, associated with the phenomenon of vasomotion have been analyzed using computational model in (Jacobsen et al. 2007). Based on their observations, they supported the role of cGMP-dependent $\mathrm{Ca}^{2+}$-activated chloride channels $\left(\mathrm{Cl}_{\mathrm{Ca}}\right)$ (Matchkov, Aalkjaer, and Nilsson 2004)in synchronizing local $\mathrm{Ca}^{2+}$ oscillators into whole-cell oscillations. According to the model, activation of chloride channels by $\mathrm{Ca}^{2+}$ transient's results in whole-cell membrane potential depolarization and $\mathrm{Ca}^{2+}$ influx via L-type voltage regulated $\mathrm{Ca}^{2+}$ channels resulting in the coordination of $\mathrm{Ca}^{2+}$ changes. Other study (Postnov et al. 2011) used a reductionist approach to reveal the dynamical features and non-linear mechanisms involved in this transition from $\mathrm{Ca}^{2+}$ waves to oscillations. Synchronization of SMCs has been investigated in a number of computational studies (Jacobsen et al. 2007; Kapela, Parikh, and Tsoukias 2012; Postnov et al. 2011; Koenigsberger et al. 2008; Jacobsen et al. 2007; 
Koenigsberger et al. 2006). The role of NSC channels in addition to the $\mathrm{Cl}^{-}$channels, and weak intercellular $\mathrm{IP}_{3}$ fluxes to promote SMCs synchronization is suggested in (Kapela, Parikh, and Tsoukias 2012). Similar to synchronization between cells, these channels may also provide pathways for transition from intracellular waves to whole-cell oscillations.

\subsubsection{Localized $\mathrm{Ca}^{2+}$ events}

Localized $\mathrm{Ca}^{2+}$ events are observed in a wide variety of cell types at rest or low agonist stimulation levels. These events are highly restricted both in space and time and originate from random opening of few SR/ER or plasmalemmal $\mathrm{Ca}^{2+}$ channels. Advancement in $\mathrm{Ca}^{2+}$ imaging techniques with the development of sensitive cameras and better dyes allow optical imaging systems to routinely achieve detailed spatial and temporal resolution, significant for characterization of local $\mathrm{Ca}^{2+}$ events. Moreover, selective loading of the ECs without interference from surrounding SMCs (Kansui, Garland, and Dora 2008), development of transgenic mouse expressing the genetically encoded $\mathrm{Ca}^{2+}$ indicator GCaMP2 (Nakai, Ohkura, and Imoto 2001; Ji et al. 2004) provide better visualization and understanding of localized $\mathrm{Ca}^{2+}$ events in the vascular ECs and SMCs. The local $\mathrm{Ca}^{2+}$ events can be classified based on whether the release is from is from a single channel or a cluster of channels, the type of channels or receptors involved such as $\mathrm{RyR}, \mathrm{IP}_{3} \mathrm{R}$ or membrane channels, and the underlying characteristics of the events. In particular, $\mathrm{Ca}^{2+}$ sparks denote localized $\mathrm{Ca}^{2+}$ increase via $\mathrm{RyR}, \mathrm{Ca}^{2+}$ puffs are localized $\mathrm{Ca}^{2+}$ events via $\mathrm{IP}_{3} \mathrm{R}, \mathrm{Ca}^{2+}$ pulsar is a localized $\mathrm{Ca}^{2+}$ increase via $\mathrm{IP}_{3} \mathrm{R}$ located in vicinity of endothelial microprojections, and $\mathrm{Ca}^{2+}$ events mediated by plasmalemmal channels are termed as $\mathrm{Ca}^{2+}$ sparklets. In vascular cells the localized events have been 
shown to modulate the vessel tone. Moreover these random local events at microscopic levels can transform into coordinated global $\mathrm{Ca}^{2+}$ responses like waves and oscillations. Examining the properties of these events, their mechanisms and transition to global $\mathrm{Ca}^{2+}$ responses will provide a more comprehensive understanding of $\mathrm{Ca}^{2+}$ dynamics.

\subsubsection{1 $\mathrm{Ca}^{2+}$ sparks (Localized $\mathrm{Ca}^{2+}$ event via RyRs)}

RyRs are expressed at varying levels in different smooth muscle cells tissues.

Dissimilar expression and/or function of three RyR subtypes is suggested to result in the diverse spatiotemporal characteristics of the $\mathrm{Ca}^{2+}$ sparks in different vascular SMCs (Zheng et al. 2008). Localized $\mathrm{Ca}^{2+}$ events via RyR termed $\mathrm{Ca}^{2+}$ sparks were first observed in cardiac cells (Cheng, Lederer, and Cannell 1993) and suggested to influence excitation-contraction coupling. These events have been reported in variety of vascular SMCs (Jaggar et al. 2000; Nelson et al. 1995; Sieck, Kannan, and Prakash 1997). Majority of the vascular ECs are found to be devoid of RyRs (Ledoux et al. 2008) except few studies which have reported their presence in the ECs and, hence may lack $\mathrm{Ca}^{2+}$ sparks. A single $\mathrm{Ca}^{2+}$ spark results in increase of local $\mathrm{Ca}^{2+}$ level to around $10-100 \mu \mathrm{M}$ (Jaggar et al. 2000) near the site of release and spread to around $14 \mu \mathrm{m}^{2}$. Other properties of $\mathrm{Ca}^{2+}$ sparks are listed in Table 1.1.

Due to their limited spread, $\mathrm{Ca}^{2+}$ sparks have been suggested to play a minimal influence on the global $\mathrm{Ca}^{2+}$ levels (Nelson et al. 1995). $\mathrm{Ca}^{2+}$ sparks occurring in regions of the cell with SR in close proximity to the plasma membrane can result in indirect regulation of contraction and relaxation of the SMC through activation of co-localized $\mathrm{Ca}^{2+}$-dependent membrane channels. $\mathrm{Ca}^{2+}$ spark can result in relaxation of rat cerebral artery SMCs (Nelson et al. 1995) through co-localization of RyR with BK $\mathrm{Ca}_{\text {a. }}$ Localized 
$\mathrm{Ca}^{2+}$ increase in the microdomain can activate several $\mathrm{BK}_{\mathrm{Ca}}$ channels, resulting in SMC hyperpolarization, global $\mathrm{Ca}^{2+}$ decrease, and in turn SMC relaxation. On contrary, in SMCs from rabbit portal vein tissues RyRs are juxta-posed with the $\mathrm{Cl}_{\mathrm{Ca}}$ and localized $\mathrm{Ca}^{2+}$ sparks in these microdomains cause SMC depolarization (Wang, Hogg, and Large 1992), global $\mathrm{Ca}^{2+}$ increase via opening of $\mathrm{L}-$ type voltage gated $\mathrm{Ca}^{2+}$ channels, and SMC contraction. Reduction in amplitude and frequency of $\mathrm{Ca}^{2+}$ sparks in SMCs from cerebral artery of type- 2 diabetes model have been reported and suggested to be involved in cerebral artery dysfunction in diabetic patients (Rueda et al. 2013).

\subsubsection{2 $\mathrm{Ca}^{2+}$ puffs (localized $\mathrm{Ca}^{2+}$ events via $I P_{3} R s$ )}

Three $\mathrm{IP}_{3} \mathrm{R}$ isoforms $\left(\mathrm{IP}_{3} \mathrm{R} 1, \mathrm{IP}_{3} \mathrm{R} 2\right.$ and $\left.\mathrm{IP}_{3} \mathrm{R} 3\right)$ are expressed at varying levels in both ECs and SMCs (Ledoux et al. 2008; Tovey et al. 2001; Tasker, Michelangeli, and Nixon 1999). Spontaneous $\mathrm{Ca}^{2+}$ release via single $\mathrm{IP}_{3} \mathrm{R}$ is referred as $\mathrm{Ca}^{2+}$ blip. $\mathrm{Ca}^{2+}$ blips in the EC have shown to last less than $100 \mathrm{~ms}$ with an average amplitude of $23 \mathrm{nmol} / \mathrm{L}$ corresponding to $0.3 \mathrm{pA}$ influx and a spread of 1- to $3-\mu \mathrm{m}$ width (Huser and Blatter 1997). Sufficiently high $\mathrm{Ca}^{2+}$ release from a single channel can activate other $\mathrm{IP}_{3} \mathrm{Rs}$ in a cluster through passive diffusion, and produce a slightly larger but still random $\mathrm{Ca}^{2+}$ event termed as $\mathrm{Ca}^{2+}$ puff. $\mathrm{Ca}^{2+}$ puffs have been observed in ECs (Kansui, Garland, and Dora 2008; Duza and Sarelius 2004, 2004). They are observed in variety of cell types with different $\mathrm{IP}_{3} \mathrm{R} 1-3$ levels with similar amplitude, spatial spread, and kinetics, and hence suggested to be generic elementary $\mathrm{Ca}^{2+}$ signals with minimal functional variation of the three $\mathrm{IP}_{3} \mathrm{R}$ isoforms at the level of subcellular $\mathrm{Ca}^{2+}$ signaling (Tovey et al. 2001).. $\mathrm{Ca}^{2+}$ puffs have not been examined as widely as $\mathrm{Ca}^{2+}$ sparks in vascular SMCs, with exception of few studies on colonic and cerebral artery SMCs (Bayguinov et al. 2000; 
Gonzales, Amberg, and Earley 2010). $\mathrm{Ca}^{2+}$ dynamics in the SMC at the macroscopic level has been suggested to be significantly modulated by the expression levels of various subtypes of $\mathrm{IP}_{3} \mathrm{Rs}$ (see $\mathrm{Ca}^{2+}$ waves section). The effect of different $\mathrm{IP}_{3} \mathrm{R}$ receptors have been reviewed in Dupont et al (Dupont, Combettes, and Leybaert 2007).

A single $\mathrm{Ca}^{2+}$ puff results in increase of local $\mathrm{Ca}^{2+}$ level to around 50-500 $\mathrm{nM}$ (Ledoux et al. 2008; Tovey et al. 2001) near the site of release. Other properties of $\mathrm{Ca}^{2+}$ puffs are listed in Table 1.1. Localized $\mathrm{Ca}^{2+}$ events via $\mathrm{IP}_{3} \mathrm{Rs}$ in vascular cells have been shown to modulate the vessel tone. The presence of microdomains where $\mathrm{IP}_{3} \mathrm{Rs}$ are co-localized with specific membrane channels TRPM4 (transient receptor potential melastatin 4), $\mathrm{IK}_{\mathrm{Ca}}$ (intermediate conductance $\mathrm{Ca}^{2+}$ activated potassium channels)) permits spatially limited $\mathrm{Ca}^{2+}$ release to be sufficient to activate these channels and modulate vessel tone. In rat cerebral SMCs $\mathrm{Ca}^{2+}$ puffs have been shown to promote opening of TRPM4 channels, resulting in SMC membrane depolarization, opening of VOCC channels and ultimately contraction. Role of $\mathrm{Ca}^{2+}$ puffs in regulation of vascular tone have been reviewed in (Amberg and Navedo 2013).

\subsubsection{3 $\mathrm{Ca}^{2+}$ pulsars and wavelets}

Spontaneous localized $\mathrm{Ca}^{2+}$ increases through $\mathrm{IP}_{3} \mathrm{Rs}$ in the EC near myoendothelial projections (MPs) are reported in unstimulated rat mesenteric arteries and referred as $\mathrm{Ca}^{2+}$ pulsars (Ledoux et al. 2008). Several studies since then have reported existence of $\mathrm{Ca}^{2+}$ pulsars at the sites of MPs (Sonkusare et al. 2012; Nausch et al. 2012; Bagher et al. 2012). MPs are cellular extensions from ECs and/or SMC that extend over the internal elastic lamina and come in close contact with the other cell type (Heberlein, Straub, and Isakson 2009; Sandow et al. 2009). Although these localized $\mathrm{Ca}^{2+}$ events are associated 
with $\mathrm{IP}_{3} \mathrm{Rs}$, their spatial spread and kinetic properties are significantly different from $\mathrm{Ca}^{2+}$ puffs and more similar to $\mathrm{Ca}^{2+}$ sparks (Table 1.1). Increased frequency of $\mathrm{Ca}^{2+}$ pulsars under acetylcholine stimulation and activation of intermediate conductance potassium channels $\left(\mathrm{IK}_{\mathrm{Ca}}\right)$ co-localized around myoendothelial projections can significantly hyperpolarize the EC (Ledoux et al. 2008) which may spread to adjacent SMCs to ultimately induce vessel relaxation.

Localized $\mathrm{Ca}^{2+}$ increase via a discrete pool of $\mathrm{IP}_{3} \mathrm{Rs}$ localized in the endothelial projections have been reported in muscle feed arteries under stimulation of the SMC with norepinephrine, and are referred as $\mathrm{Ca}^{2+}$ wavelets (Tran et al. 2012), The duration and spread of $\mathrm{Ca}^{2+}$ wavelets are similar to those of $\mathrm{Ca}^{2+}$ pulsars and significantly higher than of $\mathrm{Ca}^{2+}$ puffs (Table 1.1). The $\mathrm{Ca}^{2+}$ wavelet are suggested to activate $\mathrm{IK}_{\mathrm{Ca}}$ channels within or near endothelial projections, resulting in hyperpolarization which feeds back on the SMC to moderate agonist-induced depolarization and constriction (Kerr et al. 2012; Tran et al. 2012). Endothelial mediated feedback was initially suggested in (Dora, Doyle, and Duling 1997).

\subsubsection{4 $\mathrm{Ca}^{2+}$ sparklets and junctional $\mathrm{Ca}^{2+}$ transients}

Localized $\mathrm{Ca}^{2+}$ events via $\mathrm{Ca}^{2+}$-permeable membrane channels are usually referred as $\mathrm{Ca}^{2+}$ sparklets. $\mathrm{Ca}^{2+}$ sparklets are found to occur in persistent, specific region of the plasmalemma, unlike localized $\mathrm{Ca}^{2+}$ events from store channels, which usually occur randomly and uniformly throughout the cell. Initially $\mathrm{Ca}^{2+}$ sparklets were observed in cardiac muscle cells, where local $\mathrm{Ca}^{2+}$ increase results from opening of a single or a cluster of L-type VOCC (Wang et al. 2001). VOCC-induced $\mathrm{Ca}^{2+}$ sparklets are observed in vascular SMCs, using total internal reflection (TIRF) microscopy, and reported to 
modulate global $\mathrm{Ca}^{2+}$ levels indirectly through activation of RyRs (Navedo et al. 2005). The average area of VOCC-induced $\mathrm{Ca}^{2+}$ sparklets is small and approximately $0.8 \mu \mathrm{m}^{2}$ (Santana et al. 2008). Santana et al. described low and high activity ("persistent") $\mathrm{Ca}^{2+}$ sparklets and necessity of protein kinase $\mathrm{C}$ (PKC) activation for increased frequency of persistent sparklets. Events via VOCC channels and their role in modulation of vascular tone have been summarized in (Hill-Eubanks et al. 2011).

Localized $\mathrm{Ca}^{2+}$ events have also been observed from $\mathrm{Ca}^{2+}$-permeable non-selective cation channels (NSC) such as TPRV4 and ATP-gated P2X receptors, which allow influx of $\mathrm{Ca}^{2+}$ and other extracellular cations like $\mathrm{Na}^{+}$. Stimulation of P2X receptors by ATP revealed localized $\mathrm{Ca}^{2+}$ influx events referred as junctional $\mathrm{Ca}^{2+}$ transients have been observed in pressurized rat mesenteric small arteries (Lamont and Wier 2002). The properties of these events are described in Table 1.1. In addition to the localized $\mathrm{Ca}^{2+}$ events, activation of $\mathrm{P} 2 \mathrm{X}$ receptors may induce depolarizing excitatory junctional potential due to $\mathrm{Na}^{+}$influx, and global $\mathrm{Ca}^{2+}$ increase via activation of VOCC (Zang et al. 2006). Localized quantal $\mathrm{Ca}^{2+}$ increases through TRPV4 channels, referred as TRPV4 sparklets, have been identified recently in mice mesenteric arteries ECs, using genetically encoded $\mathrm{Ca}^{2+}$ biosensor (GCaMP2) for $\mathrm{Ca}^{2+}$ imaging (Sonkusare et al. 2012). The TRPV4 sparklets have a spatial spread of $11 \mu \mathrm{m}^{2}$ and reported to occur at the same sites. Activation of few ( $\sim 3)$ TRPV4 channels per cell is suggested to induce maximal vessel dilation through activation of co-localized $\mathrm{EC} \mathrm{IK}_{\mathrm{Ca}}$ and small-conductance $\mathrm{Ca}^{2+}$-activated potassium $\left(\mathrm{SK}_{\mathrm{Ca}}\right)$ channels. Low intraluminal pressure may also stimulate TRPV4associated $\mathrm{EC} \mathrm{Ca}^{2+}$ sparklet events (Bagher et al. 2012). AKAP150, a protein kinase $\mathrm{C}$ (PKC) anchoring protein mediated cooperative opening of a 4-channel TRPV4 cluster 
fashioned $\mathrm{Ca}^{2+}$ sparklet near the EC MPs site have been suggested to be central to endothelium dependent vasodilation during muscarinic-receptor stimulation (Sonkusare et al. 2014).

\subsubsection{Mathematical modeling}

Computational studies aim at quantification and better understanding of the characteristics of these localized $\mathrm{Ca}^{2+}$ events and their vasoregulatory mechanisms. Although the majority of models developed are based on data from nonvascular cells, their results and methodology can be also utilized to investigate the localized $\mathrm{Ca}^{2+}$ events reported in vascular ECs and SMCs. The initial models of $\mathrm{Ca}^{2+}$ sparks, puffs were simple reaction-diffusion models involving $\mathrm{Ca}^{2+}$ release from the stores (SR/ER), cytosolic $\mathrm{Ca}^{2+}$ diffusion, reaction with cytosolic $\mathrm{Ca}^{2+}$ buffers and re-sequestration of $\mathrm{Ca}^{2+}$ into the store via pumps. Point source release of $\mathrm{Ca}^{2+}$ calcium was achieved in the models using a timedependent point source $\sigma(\mu$ mole/s) times a Dirac-delta function $(\delta(\mathrm{r}))$ (Smith et al. 1998; Smith 1996). Fast buffering approximations similar to in (Wagner and Keizer 1994) were considered. Another approach have also been carried out to model localized $\mathrm{Ca}^{2+}$ puffs and sparks events using stochastic descriptions of single or cluster $\mathrm{IP}_{3} \mathrm{R}$ and $\mathrm{RyR}$ ( implemented using Markov chain modeling (Colquhoun and Hawkes 1981)) coupled with deterministic cytosolic $\mathrm{Ca}^{2+}$ diffusion, $\mathrm{Ca}^{2+}$ buffering, store uptake, and membrane $\mathrm{Ca}^{2+}$ fluxes (Swillens et al. 1998; Falcke, Tsimring, and Levine 2000; Keizer et al. 1998). Stochastic transitions among the different channel states were characterized by the number of $\mathrm{IP}_{3}$ and $\mathrm{Ca}^{2+}$ molecules bound to their respective binding sites (Swillens et al. 1998). The models are able to predict the amount of localized increase from opening of a single or cluster of channels, number of channels in a cluster, and separation distances 
between clusters. Several reviews have described in details stochastic modeling of the localized events (Dupont et al. 2000; Dupont, Combettes, and Leybaert 2007; Jafri 2012). To improve computational efficiency, some models of $\mathrm{Ca}^{2+}$ puffs implemented hybrid Gillespie method for $\mathrm{Ca}^{2+}$ - or $\mathrm{IP}_{3}$-dependent transitions, and neglected $\mathrm{Ca}^{2+}$ diffusion and spatial distributions (Keener and Sneyd 1998; Cao et al. 2013). On the other hand, computationally expensive detailed models have been formulated. For example, model of $\mathrm{Ca}^{2+}$ sparks in cardiac cells have been implemented using 3D finite elements method (FEM) with geometry imported from electron tomographic images of $\mathrm{Ca}^{2+}$ release units (CRU) (Hake et al. 2012).

Deterministic continuum models of coupled vascular EC-SMC have been developed and arising local and global $\mathrm{Ca}^{2+}$ transients examined in (Nagaraja et al. 2013; Brasen, Jacobsen, and Holstein-Rathlou 2012). Brasen et al. (Brasen, Jacobsen, and HolsteinRathlou 2012) used a 2-D axisymmetric model and predicts localized spatiotemporal $\mathrm{Ca}^{2+}$ responses in EC MP during SMC stimulation, and suggest the rectification of $\mathrm{Ca}^{2+}$ signals by microprojections geometry. A detailed 2D continuum model using finite element techniques to investigate integrated $\mathrm{Ca}^{2+}$ and $V_{\mathrm{m}}$ responses is presented in (Nagaraja et al. 2013). The model incorporates exact geometries of myoendothelial projections (MP) from experiments and localized pool of $\mathrm{IP}_{3} \mathrm{Rs}$ and $\mathrm{IK}_{\mathrm{Ca}}$. A cartoon illustration of the cellular components implemented in the model is shown in Figure 1.5. Kir, inward rectifier $\mathrm{K}^{+}$channel; VRAC, volume-regulated anion channel; $\mathrm{SK}_{\mathrm{Ca}}, \mathrm{IK}_{\mathrm{Ca}}$, and $\mathrm{BK}_{\mathrm{Ca}}$, small-, intermediate-, and large-conductance $\mathrm{Ca}^{2+}$-activated $\mathrm{K}^{+}$channels; SOC, store-operated channel; NSC, nonselective cation channel, $\mathrm{CaCC}$ and $\mathrm{ClCa}, \mathrm{Ca}^{2+}-$ activated chloride channel; $\mathrm{NaK}, \mathrm{Na}^{+}-\mathrm{K}^{+}$-ATPase; PMCA, plasma-membrane $\mathrm{Ca}^{2+}$ - 
ATPase; $\mathrm{NCX}, \mathrm{Na}^{+} / \mathrm{Ca}^{2+}$ exchanger; $\mathrm{NaKCl}, \mathrm{Na}^{+}-\mathrm{K}^{+}-\mathrm{Cl}^{-}$cotransport; $\mathrm{Kv}$, voltagedependent $\mathrm{K}^{+}$channel; $\mathrm{K}_{\text {leak}}$, unspecified $\mathrm{K}^{+}$leak current; VOCC, voltage-operated $\mathrm{Ca}^{2+}$ channels; SR/ER, sarco/endoplasmic reticulum; $\mathrm{IP}_{3} \mathrm{R}, \mathrm{IP}_{3}$ receptor; RyR, ryanodine receptor; SERCA, SR/ER $\mathrm{Ca}^{2+}$-ATPase; CSQN, calsequestrin; CM, calmodulin; R, receptor; G, G protein; DAG, diacylglycerol; PLC, phospholipase C; sGC, soluble guanylate cyclase; cGMP, cyclic guanosine monophosphate were considered in the model.

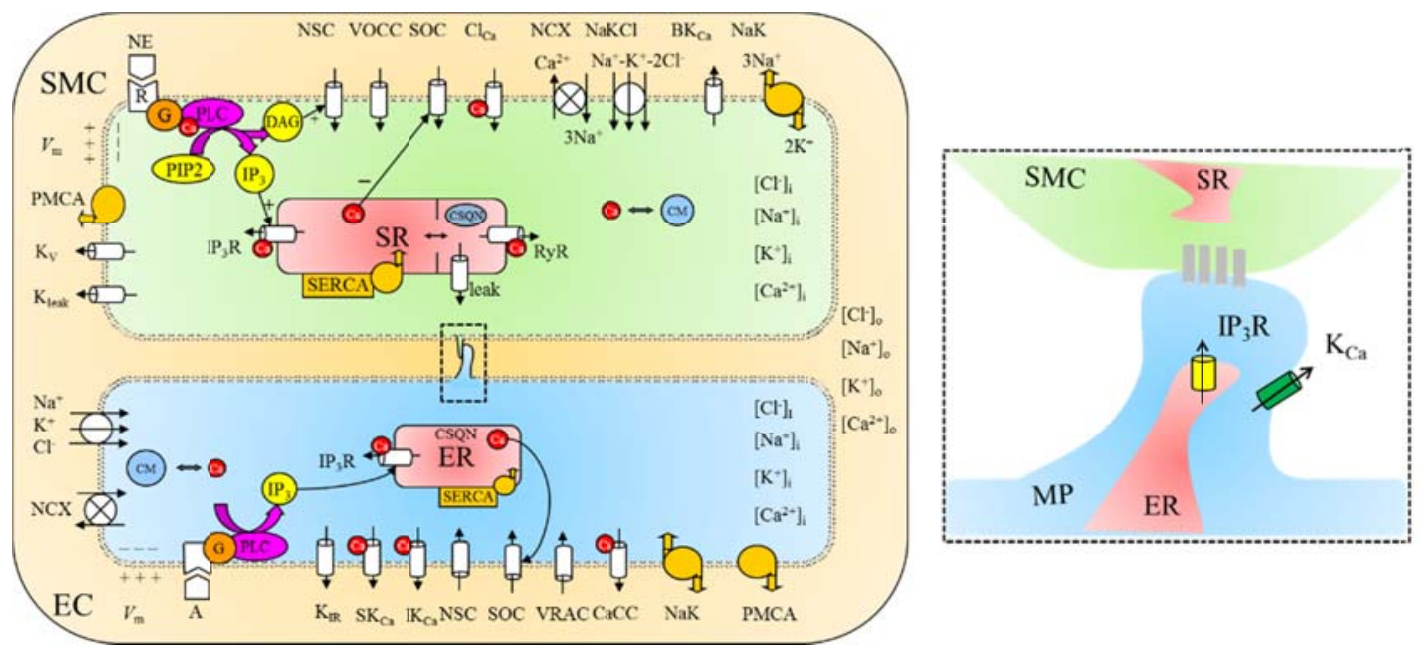

Figure 1.5 Cartoon illustration of coupled EC-SMC model with MP

Enriched distribution of $\mathrm{IK}_{\mathrm{Ca}}$ and $\mathrm{IP}_{3} \mathrm{Rs}$ around the MP was implemented in the model and the cells were coupled by myoendothelial gap junctions (MEGJ) permeable to $\mathrm{Ca}^{2+}$, $\mathrm{Na}^{+}, \mathrm{K}^{+}$, and $\mathrm{Cl}^{-}$ions and $\mathrm{IP}_{3}$. The model simulations predicted local $\mathrm{Ca}^{2+}$ release in and around EC MP and activation of spatially localized $\mathrm{IK}_{\mathrm{Ca}}$ channels to produce hyperpolarizing currents which can moderate smooth muscle constriction. Few $\mathrm{mV}$ of feedback was observed in the model during SMC stimulation. Global $\mathrm{EC} \mathrm{Ca}^{2+}$ response was not observed during SMC stimulation. 


\subsubsection{Integrated cytosolic and membrane potential modeling}

The above review of the variety of spatiotemporal $\mathrm{Ca}^{2+}$ patterns demonstrates that many $\mathrm{Ca}^{2+}$ events are tightly coupled with changes in membrane potential. For example, $V_{\mathrm{m}}$ depolarization may increase the activity of $\mathrm{Ca}^{2+}$ sparklets through VOCC and activate CICR through RyRs, which in turn can feedback on $V_{\mathrm{m}}$ through $\mathrm{Ca}^{2+}$-activated membrane channels.

A general computational framework for modeling spatiotemporal $\mathrm{Ca}^{2+}$ events integrated with plasma membrane electrophysiology in single or coupled vascular cells has been proposed in (Kapela and Tsoukias 2011). It is based on the FEM approach, and allows implementing continuous models (as opposed to discrete compartmental models) in full three dimensions, two dimensions with axial symmetry, two dimensions with lumped third dimension, or only one dimension. Transport of ionic species $S=\mathrm{Ca}^{2+}, \mathrm{Na}^{+}, \mathrm{K}^{+}, \mathrm{Cl}^{-}$across the cell cytosol is given by the electrodiffusion equation,

$$
\frac{\partial[S]_{i}}{\partial t}=\nabla \cdot\left(D_{S} \nabla[S]_{i}+Z_{s} F u_{m s}[S]_{i} \nabla V_{i}+I_{r e l, S}\right)
$$

\section{Equation 1.1}

where $D_{\mathrm{S}}$ is the diffusion coefficient of ionic species, $u_{\mathrm{mS}}$ is the ionic mobility, $\mathrm{Z}_{\mathrm{s}}$ is the charge number of ionic species $S, I_{\mathrm{rel}, \mathrm{S}}$ is the net release and uptake flux of ion $S$ from the internal stores $\left(I_{\text {rel,S }}=0\right.$ for $S=\mathrm{Na}^{+}, \mathrm{K}^{+}$, and $\left.\mathrm{Cl}^{-}\right)$. The ionic currents through membrane channels, pumps, and exchangers can be implemented as boundary fluxes. The net boundary flux $\left(N_{S}\right)$ (Equation 1.2) for each ionic species $S$ is calculated from the sum of $K$ component currents $\left(I_{S, K}\right)$, corresponding to all channel types permeable to $S$. The FEM approach allows introduction of spatial heterogeneity of cellular components by defining 
the maximum conductance of release and membrane fluxes as functions of spatial coordinates. Moreover, 2D or 3D FEM models allow incorporation of microdomain structures with geometries imported from experimental images, e.x. electron or confocal microscopy.

$$
-n \cdot N_{S}=\frac{1}{Z_{S} F} \sum_{K} I_{S, K}
$$

Equation 1.2

Electrodiffusion equation (Equation 1.1) takes into account the effect of both concentration and electrical potential gradients across the cell cytosol on the transport of all the ionic species. Although the cytosol is largely isopotential, the electrodiffusion equation can capture possible heterogeneities in electrical potential between cytosol and some microdomains generated during current conduction and limited bulk-microdomain diffusivity, e.x. in endothelial microprojection during EDHF response. The electrodiffusion model in the cytosol also allows simple implementation of voltage and concentration dependent membrane currents as boundary fluxes. Furthermore, the use of electrodiffusion equations, rather than diffusion equations, permits implementation of intercellular coupling through gap junctions as ionic currents proportional to electrochemical gradients. The implementation of intercellular coupling and the role of intercellular fluxes have been discussed in detail in Nagaraja et al. (Nagaraja, Kapela, and Tsoukias 2012). The release and membrane currents in Equations.1.1 and 1.2 can be implemented as stochastic processes, rather than with deterministic equations, to study localized events such as $\mathrm{Ca}^{2+}$ puffs, sparks and sparklets. 


\subsection{Outline of the dissertation}

- Chapter 2 presents the formulation of a coupled stochastic-continuum model of integrated calcium $\left(\mathrm{Ca}^{2+}\right)$ and membrane potential $\left(V_{m}\right)$ dynamics in combined endothelial (EC) and smooth muscle (SMC) cell domains with microprojections (MP). The model describes the localized $\mathrm{Ca}^{2+}$ events arising from stochastic opening of a cluster of TRPV4 channels in the EC and its contribution to the regulation of vessel tone.

- Chapter 3 describes the development of a detailed continuum model of coupled EC-SMC with MP, incorporated with the NO-cGMP pathway. We examine the NO mediated vasodilatory feedback response. The model predicts local NO production under the preferential distribution of endothelial nitric oxide synthase (eNOS) around the MP domain. Moreover, the model examines the modulation of SMC NO bioavailability in the presence of $\mathrm{NO}$ scavengers such as endothelial Hemoglobin $\alpha(\mathrm{Hb} \alpha)$ and erythrocytes.

- Chapter 4 presents the development of individual spatiotemporal models of isolated SMC and the EC to examine the repetitive intracellular $\mathrm{Ca}^{2+}$ wave activity frequently observed in both the cell types. It also discusses the role of heterogeneity of cellular components, diffusion of $\mathrm{Ca}^{2+}$ and inositol triphosphate $\left(\mathrm{IP}_{3}\right)$, and electrical signaling $\left(V_{\mathrm{m}}\right.$ modulation $)$ in the generation of oscillatory $\mathrm{Ca}^{2+}$ waves. Moreover it examines the effects of membrane potential dynamics on the modulation of intracellular $\mathrm{Ca}^{2+}$ wave frequency and velocity. 
- Chapter 5 provides a multi-cellular temporal model of a vessel segment (consisting of a layer of ECs with multiple layer of SMCs) and describes the role of $\mathrm{Ca}^{2+}$ and second messenger $\left(\mathrm{IP}_{3}\right)$ gap junction fluxes and electrical coupling in synchronizing SMC $\mathrm{Ca}^{2+}$ oscillations and producing oscillations in vessel diameter.

- Chapter 6 provides a summary of the dissertation and,

- Chapter 7 lists the equations of the models.

\subsection{References}

Amberg, G. C., and M. F. Navedo. 2013. Calcium dynamics in vascular smooth muscle. Microcirculation 20 (4):281-9.

Atri, A., J. Amundson, D. Clapham, and J. Sneyd. 1993. A single-pool model for intracellular calcium oscillations and waves in the Xenopus laevis oocyte. Biophys J 65 (4):1727-39.

Bagher, P., T. Beleznai, Y. Kansui, R. Mitchell, C. J. Garland, and K. A. Dora. 2012. Low intravascular pressure activates endothelial cell TRPV4 channels, local $\mathrm{Ca} 2+$ events, and IKCa channels, reducing arteriolar tone. Proc Natl Acad Sci U S A 109 (44):18174-9.

Bayguinov, O., B. Hagen, A. D. Bonev, M. T. Nelson, and K. M. Sanders. 2000. Intracellular calcium events activated by ATP in murine colonic myocytes. Am J Physiol Cell Physiol 279 (1):C126-35.

Beliveau, E., F. Lapointe, and G. Guillemette. 2011. The activation state of the inositol 1,4,5-trisphosphate receptor regulates the velocity of intracellular $\mathrm{Ca} 2+$ waves in bovine aortic endothelial cells. J Cell Biochem 112 (12):3722-31.

Berridge, M. J. 1993. Inositol trisphosphate and calcium signalling. Nature 361 (6410):315-25.

Berridge, M. J. 1997. Elementary and global aspects of calcium signalling. J Physiol 499 ( Pt 2):291-306.

Berridge, M. J., M. D. Bootman, and H. L. Roderick. 2003. Calcium signalling: dynamics, homeostasis and remodelling. Nat Rev Mol Cell Biol 4 (7):517-29. 
Berridge, M. J., and G. Dupont. 1994. Spatial and temporal signalling by calcium. Curr Opin Cell Biol 6 (2):267-74.

Berridge, M. J., and A. Galione. 1988. Cytosolic calcium oscillators. FASEB J 2 (15):3074-82.

Berridge, M. J., and R. F. Irvine. 1989. Inositol phosphates and cell signalling. Nature 341 (6239):197-205.

Berridge, M. J., P. Lipp, and M. D. Bootman. 2000. The versatility and universality of calcium signalling. Nat Rev Mol Cell Biol 1 (1):11-21.

Bezprozvanny, I. 1994. Theoretical analysis of calcium wave propagation based on inositol (1,4,5)-trisphosphate (InsP3) receptor functional properties. Cell Calcium $16(3): 151-66$.

Bezprozvanny, I., J. Watras, and B. E. Ehrlich. 1991. Bell-shaped calcium-response curves of Ins $(1,4,5) \mathrm{P} 3$ - and calcium-gated channels from endoplasmic reticulum of cerebellum. Nature 351 (6329):751-4.

Boittin, F. X., N. Macrez, G. Halet, and J. Mironneau. 1999. Norepinephrine-induced $\mathrm{Ca}(2+)$ waves depend on $\mathrm{InsP}(3)$ and ryanodine receptor activation in vascular myocytes. Am J Physiol 277 (1 Pt 1):C139-51.

Brasen, J. C., J. C. Jacobsen, and N. H. Holstein-Rathlou. 2012. The nanostructure of myoendothelial junctions contributes to signal rectification between endothelial and vascular smooth muscle cells. PloS one 7 (4):e33632.

Buerk, D. G., and C. E. Riva. 1998. Vasomotion and spontaneous low-frequency oscillations in blood flow and nitric oxide in cat optic nerve head. Microvasc Res 55 (1):103-12.

Cao, P., G. Donovan, M. Falcke, and J. Sneyd. 2013. A stochastic model of calcium puffs based on single-channel data. Biophys $J 105$ (5):1133-42.

Chen, G., H. Suzuki, and A. H. Weston. 1988. Acetylcholine releases endotheliumderived hyperpolarizing factor and EDRF from rat blood vessels. Br J Pharmacol 95 (4):1165-74.

Cheng, H., W. J. Lederer, and M. B. Cannell. 1993. Calcium sparks: elementary events underlying excitation-contraction coupling in heart muscle. Science 262 (5134):740-4.

Colquhoun, D., and A. G. Hawkes. 1981. On the stochastic properties of single ion channels. Proc R Soc Lond B Biol Sci 211 (1183):205-35. 
Combettes, L., G. Dupont, and J. B. Parys. 2004. New mechanisms and functions in $\mathrm{Ca} 2+$ signalling. Biol Cell 96 (1):1-2.

Dai, J., C. H. Lee, D. Poburko, T. Szado, K. H. Kuo, and C. van Breemen. 2007. Endothelin-1-mediated wave-like [Ca2+]i oscillations in intact rabbit inferior vena cava. $J$ Vasc Res 44 (6):495-503.

Dai, J. M., K. H. Kuo, J. M. Leo, C. van Breemen, and C. H. Lee. 2006. Mechanism of ACh-induced asynchronous calcium waves and tonic contraction in porcine tracheal muscle bundle. Am J Physiol Lung Cell Mol Physiol 290 (3):L459-69.

De Koninck, P., and H. Schulman. 1998. Sensitivity of CaM kinase II to the frequency of Ca2+ oscillations. Science 279 (5348):227-30.

De Young, G. W., and J. Keizer. 1992. A single-pool inositol 1,4,5-trisphosphatereceptor-based model for agonist-stimulated oscillations in $\mathrm{Ca} 2+$ concentration. Proc Natl Acad Sci U S A 89 (20):9895-9.

Dolmetsch, R. E., K. Xu, and R. S. Lewis. 1998. Calcium oscillations increase the efficiency and specificity of gene expression. Nature 392 (6679):933-6.

Dora, K. A., M. P. Doyle, and B. R. Duling. 1997. Elevation of intracellular calcium in smooth muscle causes endothelial cell generation of NO in arterioles. Proc Natl Acad Sci U S A 94 (12):6529-34.

Dora, K. A., J. M. Hinton, S. D. Walker, and C. J. Garland. 2000. An indirect influence of phenylephrine on the release of endothelium-derived vasodilators in rat small mesenteric artery. Br J Pharmacol 129 (2):381-7.

Dupont, G., and L. Combettes. 2006. Modelling the effect of specific inositol 1,4,5trisphosphate receptor isoforms on cellular Ca2+ signals. Biol Cell 98 (3):171-82.

Dupont, G., L. Combettes, G. S. Bird, and J. W. Putney. 2011. Calcium oscillations. Cold Spring Harb Perspect Biol 3 (3).

Dupont, G., L. Combettes, and L. Leybaert. 2007. Calcium dynamics: spatio-temporal organization from the subcellular to the organ level. Int Rev Cytol 261:193-245.

Dupont, G., and A. Goldbeter. 1994. Properties of intracellular Ca2+ waves generated by a model based on $\mathrm{Ca}(2+)$-induced Ca2+ release. Biophys $J 67$ (6):2191-204.

Dupont, G., S. Swillens, C. Clair, T. Tordjmann, and L. Combettes. 2000. Hierarchical organization of calcium signals in hepatocytes: from experiments to models. Biochim Biophys Acta 1498 (2-3):134-52.

Duza, T., and I. H. Sarelius. 2004. Increase in endothelial cell $\mathrm{Ca}(2+)$ in response to mouse cremaster muscle contraction. J Physiol 555 (Pt 2):459-69. 
Duza, T., and I. H. Sarelius. 2004. Localized transient increases in endothelial cell Ca2+ in arterioles in situ: implications for coordination of vascular function. $\mathrm{Am} \mathrm{J}$ Physiol Heart Circ Physiol 286 (6):H2322-31.

Falcke, M. 2003. On the role of stochastic channel behavior in intracellular $\mathrm{Ca} 2+$ dynamics. Biophys $J 84$ (1):42-56.

Falcke, M., L. Tsimring, and H. Levine. 2000. Stochastic spreading of intracellular $\mathrm{Ca}(2+)$ release. Phys Rev E Stat Phys Plasmas Fluids Relat Interdiscip Topics 62 (2 Pt B):2636-43.

Falcke, Martin. 2004. Reading the patterns in living cells — the physics of ca2+ signaling. Advances in Physics 53 (3):255-440.

Fioretti, B., F. Franciolini, and L. Catacuzzeno. 2005. A model of intracellular Ca2+ oscillations based on the activity of the intermediate-conductance $\mathrm{Ca} 2+$-activated $\mathrm{K}+$ channels. Biophys Chem 113 (1):17-23.

Flower, R. J. 2006. Prostaglandins, bioassay and inflammation. Br J Pharmacol 147 Suppl 1:S182-92.

Furchgott, R. F., and J. V. Zawadzki. 1980. The obligatory role of endothelial cells in the relaxation of arterial smooth muscle by acetylcholine. Nature 288 (5789):373-6.

Garland, C. J., C. R. Hiley, and K. A. Dora. 2011. EDHF: spreading the influence of the endothelium. Br J Pharmacol 164 (3):839-52.

Gonzales, A. L., G. C. Amberg, and S. Earley. 2010. Ca2+ release from the sarcoplasmic reticulum is required for sustained TRPM4 activity in cerebral artery smooth muscle cells. Am J Physiol Cell Physiol 299 (2):C279-88.

Hake, J., A. G. Edwards, Z. Yu, P. M. Kekenes-Huskey, A. P. Michailova, J. A. McCammon, M. J. Holst, M. Hoshijima, and A. D. McCulloch. 2012. Modelling cardiac calcium sparks in a three-dimensional reconstruction of a calcium release unit. J Physiol 590 (Pt 18):4403-22.

Halidi, N., F. X. Boittin, J. L. Beny, and J. J. Meister. 2011. Propagation of fast and slow intercellular $\mathrm{Ca}(2+)$ waves in primary cultured arterial smooth muscle cells. Cell Calcium 50 (5):459-67.

Heberlein, K. R., A. C. Straub, and B. E. Isakson. 2009. The myoendothelial junction: breaking through the matrix? Microcirculation 16 (4):307-22.

Heppner, T. J., A. D. Bonev, L. F. Santana, and M. T. Nelson. 2002. Alkaline pH shifts $\mathrm{Ca} 2+$ sparks to $\mathrm{Ca} 2+$ waves in smooth muscle cells of pressurized cerebral arteries. Am J Physiol Heart Circ Physiol 283 (6):H2169-76. 
Hill-Eubanks, D. C., M. E. Werner, T. J. Heppner, and M. T. Nelson. 2011. Calcium signaling in smooth muscle. Cold Spring Harb Perspect Biol 3 (9):a004549.

Huser, J., and L. A. Blatter. 1997. Elementary events of agonist-induced Ca2+ release in vascular endothelial cells. Am J Physiol 273 (5 Pt 1):C1775-82.

Ignarro, L. J., G. M. Buga, K. S. Wood, R. E. Byrns, and G. Chaudhuri. 1987. Endothelium-derived relaxing factor produced and released from artery and vein is nitric oxide. Proc Natl Acad Sci U S A 84 (24):9265-9.

Iino, M, H Kasai, and T Yamazawa. 1994. Visualization of neural control of intracellular $\mathrm{Ca} 2+$ concentration in single vascular smooth muscle cells in situ. The EMBO journal 13 (21):5026.

Ishii, K., K. Hirose, and M. Iino. 2006. Ca2+ shuttling between endoplasmic reticulum and mitochondria underlying Ca2+ oscillations. EMBO Rep 7 (4):390-6.

Isshiki, M., J. Ando, R. Korenaga, H. Kogo, T. Fujimoto, T. Fujita, and A. Kamiya. 1998. Endothelial $\mathrm{Ca} 2+$ waves preferentially originate at specific loci in caveolin-rich cell edges. Proc Natl Acad Sci U S A 95 (9):5009-14.

Jacob, R., J. E. Merritt, T. J. Hallam, and T. J. Rink. 1988. Repetitive spikes in cytoplasmic calcium evoked by histamine in human endothelial cells. Nature 335 (6185):40-5.

Jacobsen, J. C., C. Aalkjaer, H. Nilsson, V. V. Matchkov, J. Freiberg, and N. H. HolsteinRathlou. 2007. Activation of a cGMP-sensitive calcium-dependent chloride channel may cause transition from calcium waves to whole cell oscillations in smooth muscle cells. Am J Physiol Heart Circ Physiol 293 (1):H215-28.

Jacobsen, J. C., C. Aalkjaer, H. Nilsson, V. V. Matchkov, J. Freiberg, and N. H. HolsteinRathlou. 2007. A model of smooth muscle cell synchronization in the arterial wall. Am J Physiol Heart Circ Physiol 293 (1):H229-37.

Jaffe, L. F. 2010. Fast calcium waves. Cell Calcium 48 (2-3):102-13.

Jafri, M. S. 2012. Models of excitation-contraction coupling in cardiac ventricular myocytes. Methods Mol Biol 910:309-35.

Jafri, M. S., and J. Keizer. 1994. Diffusion of inositol 1,4,5-trisphosphate but not Ca2+ is necessary for a class of inositol 1,4,5-trisphosphate-induced Ca2+ waves. Proc Natl Acad Sci U S A 91 (20):9485-9.

Jaggar, J. H. 2001. Intravascular pressure regulates local and global $\mathrm{Ca}(2+)$ signaling in cerebral artery smooth muscle cells. Am J Physiol Cell Physiol 281 (2):C439-48. 
Jaggar, J. H., and M. T. Nelson. 2000. Differential regulation of Ca(2+) sparks and $\mathrm{Ca}(2+)$ waves by UTP in rat cerebral artery smooth muscle cells. Am J Physiol Cell Physiol 279 (5):C1528-39.

Jaggar, J. H., V. A. Porter, W. J. Lederer, and M. T. Nelson. 2000. Calcium sparks in smooth muscle. Am J Physiol Cell Physiol 278 (2):C235-56.

Ji, G., M. E. Feldman, K. Y. Deng, K. S. Greene, J. Wilson, J. C. Lee, R. C. Johnston, M. Rishniw, Y. Tallini, J. Zhang, W. G. Wier, M. P. Blaustein, H. B. Xin, J. Nakai, and M. I. Kotlikoff. 2004. Ca2+-sensing transgenic mice: postsynaptic signaling in smooth muscle. J Biol Chem 279 (20):21461-8.

Kanai, A. J., H. C. Strauss, G. A. Truskey, A. L. Crews, S. Grunfeld, and T. Malinski. 1995. Shear stress induces ATP-independent transient nitric oxide release from vascular endothelial cells, measured directly with a porphyrinic microsensor. Circ Res 77 (2):284-93.

Kansui, Y., C. J. Garland, and K. A. Dora. 2008. Enhanced spontaneous Ca2+ events in endothelial cells reflect signalling through myoendothelial gap junctions in pressurized mesenteric arteries. Cell Calcium 44 (2):135-46.

Kapela, A., A. Bezerianos, and N. M. Tsoukias. 2008. A mathematical model of Ca2+ dynamics in rat mesenteric smooth muscle cell: agonist and NO stimulation. $J$ Theor Biol 253 (2):238-60.

Kapela, A., J. Parikh, and N. M. Tsoukias. 2012. Multiple factors influence calcium synchronization in arterial vasomotion. Biophys $J 102$ (2):211-20.

Kapela, A., and N. M. Tsoukias. 2011. Multiscale FEM modeling of vascular tone: from membrane currents to vessel mechanics. IEEE Trans Biomed Eng 58 (12):3456-9.

Keener, James P., and James Sneyd. 1998. Mathematical physiology, Interdisciplinary applied mathematics. New York: Springer.

Keizer, J., and L. Levine. 1996. Ryanodine receptor adaptation and Ca2+(-)induced Ca2+ release-dependent Ca2+ oscillations. Biophys $J 71$ (6):3477-87.

Keizer, J., G. D. Smith, S. Ponce-Dawson, and J. E. Pearson. 1998. Saltatory propagation of $\mathrm{Ca} 2+$ waves by $\mathrm{Ca} 2+$ sparks. Biophys $J 75$ (2):595-600.

Kerr, P. M., R. Tam, K. Ondrusova, R. Mittal, D. Narang, C. H. Tran, D. G. Welsh, and F. Plane. 2012. Endothelial feedback and the myoendothelial projection. Microcirculation 19 (5):416-22.

Koenigsberger, M., R. Sauser, J. L. Beny, and J. J. Meister. 2006. Effects of arterial wall stress on vasomotion. Biophys $J 91$ (5):1663-74. 
Koenigsberger, M., R. Sauser, M. Lamboley, J. L. Beny, and J. J. Meister. 2004. Ca2+ dynamics in a population of smooth muscle cells: modeling the recruitment and synchronization. Biophys $J 87$ (1):92-104.

Koenigsberger, M., R. Sauser, D. Seppey, J. L. Beny, and J. J. Meister. 2008. Calcium dynamics and vasomotion in arteries subject to isometric, isobaric, and isotonic conditions. Biophys $J 95$ (6):2728-38.

Lamont, C., and W. G. Wier. 2002. Evoked and spontaneous purinergic junctional Ca2+ transients (jCaTs) in rat small arteries. Circ Res 91 (6):454-6.

Ledoux, J., M. S. Taylor, A. D. Bonev, R. M. Hannah, V. Solodushko, B. Shui, Y. Tallini, M. I. Kotlikoff, and M. T. Nelson. 2008. Functional architecture of inositol 1,4,5-trisphosphate signaling in restricted spaces of myoendothelial projections. Proc Natl Acad Sci U S A 105 (28):9627-32.

Lee, C. H., D. Poburko, K. H. Kuo, C. Y. Seow, and C. van Breemen. 2002. Ca(2+) oscillations, gradients, and homeostasis in vascular smooth muscle. Am J Physiol Heart Circ Physiol 282 (5):H1571-83.

Lee, C. H., D. Poburko, P. Sahota, J. Sandhu, D. O. Ruehlmann, and C. van Breemen. 2001. The mechanism of phenylephrine-mediated $[\mathrm{Ca}(2+)](\mathrm{i})$ oscillations underlying tonic contraction in the rabbit inferior vena cava. J Physiol $534(\mathrm{Pt}$ 3):641-50.

Li, P., W. Wei, X. Cai, C. Soeller, M. B. Cannell, and A. V. Holden. 2010. Computational modelling of the initiation and development of spontaneous intracellular Ca2+ waves in ventricular myocytes. Philos Trans A Math Phys Eng Sci 368 (1925):3953-65.

Marchant, J., N. Callamaras, and I. Parker. 1999. Initiation of IP(3)-mediated Ca(2+) waves in Xenopus oocytes. EMBO J 18 (19):5285-99.

Matchkov, V. V., C. Aalkjaer, and H. Nilsson. 2004. A cyclic GMP-dependent calciumactivated chloride current in smooth-muscle cells from rat mesenteric resistance arteries. J Gen Physiol 123 (2):121-34.

Mauban, J. R., C. Lamont, C. W. Balke, and W. G. Wier. 2001. Adrenergic stimulation of rat resistance arteries affects $\mathrm{Ca}(2+)$ sparks, $\mathrm{Ca}(2+)$ waves, and $\mathrm{Ca}(2+)$ oscillations. Am J Physiol Heart Circ Physiol 280 (5):H2399-405.

McCarron, J. G., S. Chalmers, D. MacMillan, and M. L. Olson. 2010. Agonist-evoked $\mathrm{Ca}(2+)$ wave progression requires $\mathrm{Ca}(2+)$ and $\mathrm{IP}(3) . J$ Cell Physiol 224 (2):33444.

Meyer, T., and L. Stryer. 1988. Molecular model for receptor-stimulated calcium spiking. Proc Natl Acad Sci U S A 85 (14):5051-5. 
Moccia, F., R. Berra-Romani, and F. Tanzi. 2012. Update on vascular endothelial $\mathrm{Ca}(2+)$ signalling: A tale of ion channels, pumps and transporters. World J Biol Chem 3 (7):127-58.

Moccia, F., R. Berra-Romani, S. Tritto, S. Signorelli, V. Taglietti, and F. Tanzi. 2003. Epidermal growth factor induces intracellular $\mathrm{Ca} 2+$ oscillations in microvascular endothelial cells. J Cell Physiol 194 (2):139-50.

Mufti, R. E., S. E. Brett, C. H. Tran, R. Abd El-Rahman, Y. Anfinogenova, A. El-Yazbi, W. C. Cole, P. P. Jones, S. R. Chen, and D. G. Welsh. 2010. Intravascular pressure augments cerebral arterial constriction by inducing voltage-insensitive Ca2+ waves. J Physiol 588 (Pt 20):3983-4005.

Nagaraja, S., A. Kapela, C. H. Tran, D. G. Welsh, and N. M. Tsoukias. 2013. Role of microprojections in myoendothelial feedback--a theoretical study. J Physiol 591 (Pt 11):2795-812.

Nagaraja, S., A. Kapela, and N. M. Tsoukias. 2012. Intercellular communication in the vascular wall: a modeling perspective. Microcirculation 19 (5):391-402.

Nakai, J., M. Ohkura, and K. Imoto. 2001. A high signal-to-noise $\mathrm{Ca}(2+)$ probe composed of a single green fluorescent protein. Nat Biotechnol 19 (2):137-41.

Narayanan, D., A. Adebiyi, and J. H. Jaggar. 2012. Inositol trisphosphate receptors in smooth muscle cells. Am J Physiol Heart Circ Physiol 302 (11):H2190-210.

Nausch, L. W., A. D. Bonev, T. J. Heppner, Y. Tallini, M. I. Kotlikoff, and M. T. Nelson. 2012. Sympathetic nerve stimulation induces local endothelial $\mathrm{Ca} 2+$ signals to oppose vasoconstriction of mouse mesenteric arteries. Am J Physiol Heart Circ Physiol 302 (3):H594-602.

Navedo, M. F., G. C. Amberg, V. S. Votaw, and L. F. Santana. 2005. Constitutively active L-type Ca2+ channels. Proc Natl Acad Sci U S A 102 (31):11112-7.

Nelson, M. T., H. Cheng, M. Rubart, L. F. Santana, A. D. Bonev, H. J. Knot, and W. J. Lederer. 1995. Relaxation of arterial smooth muscle by calcium sparks. Science 270 (5236):633-7.

Neylon, C. B., and R. F. Irvine. 1990. Synchronized repetitive spikes in cytoplasmic calcium in confluent monolayers of human umbilical vein endothelial cells. FEBS Lett 275 (1-2):173-6.

Olson, M. L., M. E. Sandison, S. Chalmers, and J. G. McCarron. 2012. Microdomains of muscarinic acetylcholine and $\operatorname{Ins}(1,4,5) \mathrm{P}(3)$ receptors create $\operatorname{Ins}(1,4,5) \mathrm{P}(3)$ junctions' and sites of $\mathrm{Ca}(2)+$ wave initiation in smooth muscle. $J$ Cell Sci $125(\mathrm{Pt}$ 22):5315-28. 
Omote, M., N. Kajimoto, and H. Mizusawa. 1992. Phenylephrine induces endotheliumindependent rhythmic contraction in rabbit mesenteric arteries treated with ryanodine. Acta Physiol Scand 145 (3):295-6.

Palmer, R. M., A. G. Ferrige, and S. Moncada. 1987. Nitric oxide release accounts for the biological activity of endothelium-derived relaxing factor. Nature 327 (6122):524-6.

Peng, H., V. Matchkov, A. Ivarsen, C. Aalkjaer, and H. Nilsson. 2001. Hypothesis for the initiation of vasomotion. Circ Res 88 (8):810-5.

Postnov, D. E., J. C. Jacobsen, N. H. Holstein-Rathlou, and O. V. Sosnovtseva. 2011. Functional modeling of the shift in cellular calcium dynamics at the onset of synchronization in smooth muscle cells. Bull Math Biol 73 (10):2507-25.

Roger, V. L., A. S. Go, D. M. Lloyd-Jones, E. J. Benjamin, J. D. Berry, W. B. Borden, D. M. Bravata, S. Dai, E. S. Ford, C. S. Fox, H. J. Fullerton, C. Gillespie, S. M. Hailpern, J. A. Heit, V. J. Howard, B. M. Kissela, S. J. Kittner, D. T. Lackland, J. H. Lichtman, L. D. Lisabeth, D. M. Makuc, G. M. Marcus, A. Marelli, D. B. Matchar, C. S. Moy, D. Mozaffarian, M. E. Mussolino, G. Nichol, N. P. Paynter, E. Z. Soliman, P. D. Sorlie, N. Sotoodehnia, T. N. Turan, S. S. Virani, N. D. Wong, D. Woo, and M. B. Turner. 2012. Heart disease and stroke statistics--2012 update: a report from the American Heart Association. Circulation 125 (1):e2e220.

Rottingen, J., and J. G. Iversen. 2000. Ruled by waves? Intracellular and intercellular calcium signalling. Acta Physiol Scand 169 (3):203-19.

Rueda, A., M. Fernández-Velasco, J. P. Benitah, and A. M. Gómez. 2013. Abnormal $\mathrm{Ca} 2+$ spark/STOC coupling in cerebral artery smooth muscle cells of obese type 2 diabetic mice. PloS one 8 (1):e53321.

Ruehlmann, D. O., C. H. Lee, D. Poburko, and C. van Breemen. 2000. Asynchronous $\mathrm{Ca}(2+)$ waves in intact venous smooth muscle. Circ Res 86 (4):E72-9.

Sanders, K. M. 2001. Invited review: mechanisms of calcium handling in smooth muscles. J Appl Physiol (1985) 91 (3):1438-49.

Sandow, S. L., R. E. Haddock, C. E. Hill, P. S. Chadha, P. M. Kerr, D. G. Welsh, and F. Plane. 2009. What's where and why at a vascular myoendothelial microdomain signalling complex. Clin Exp Pharmacol Physiol 36 (1):67-76.

Santana, L. F., M. F. Navedo, G. C. Amberg, M. Nieves-Cintron, V. S. Votaw, and C. A. Ufret-Vincenty. 2008. Calcium sparklets in arterial smooth muscle. Clin Exp Pharmacol Physiol 35 (9):1121-6. 
Schuster, S., M. Marhl, and T. Hofer. 2002. Modelling of simple and complex calcium oscillations. From single-cell responses to intercellular signalling. Eur J Biochem 269 (5):1333-55.

Sieck, G. C., M. S. Kannan, and Y. S. Prakash. 1997. Heterogeneity in dynamic regulation of intracellular calcium in airway smooth muscle cells. Can J Physiol Pharmacol 75 (7):878-88.

Silva, H. S., A. Kapela, and N. M. Tsoukias. 2007. A mathematical model of plasma membrane electrophysiology and calcium dynamics in vascular endothelial cells. Am J Physiol Cell Physiol 293 (1):C277-93.

Skupin, A., H. Kettenmann, U. Winkler, M. Wartenberg, H. Sauer, S. C. Tovey, C. W. Taylor, and M. Falcke. 2008. How does intracellular Ca2+ oscillate: by chance or by the clock? Biophys $J 94$ (6):2404-11.

Smith, G. D. 1996. Analytical steady-state solution to the rapid buffering approximation near an open Ca2+ channel. Biophys $J 71$ (6):3064-72.

Smith, G. D., J. E. Keizer, M. D. Stern, W. J. Lederer, and H. Cheng. 1998. A simple numerical model of calcium spark formation and detection in cardiac myocytes. Biophys J 75 (1):15-32.

Sneyd, J., B. T. Wetton, A. C. Charles, and M. J. Sanderson. 1995. Intercellular calcium waves mediated by diffusion of inositol trisphosphate: a two-dimensional model. Am J Physiol 268 (6 Pt 1):C1537-45.

Socha, M. J., T. L. Domeier, E. J. Behringer, and S. S. Segal. 2012. Coordination of intercellular ca(2+) signaling in endothelial cell tubes of mouse resistance arteries. Microcirculation 19 (8):757-70.

Sonkusare, S. K., A. D. Bonev, J. Ledoux, W. Liedtke, M. I. Kotlikoff, T. J. Heppner, D. C. Hill-Eubanks, and M. T. Nelson. 2012. Elementary Ca2+ signals through endothelial TRPV4 channels regulate vascular function. Science 336 (6081):597601 .

Sonkusare, S. K., T. Dalsgaard, A. D. Bonev, D. C. Hill-Eubanks, M. I. Kotlikoff, J. D. Scott, L. F. Santana, and M. T. Nelson. 2014. AKAP150-dependent cooperative TRPV4 channel gating is central to endothelium-dependent vasodilation and is disrupted in hypertension. Sci Signal 7 (333):ra66.

Stewart, M., M. Needham, P. Bankhead, T. A. Gardiner, C. N. Scholfield, T. M. Curtis, and J. G. McGeown. 2012. Feedback via $\mathrm{Ca}(2)(+)$-activated ion channels modulates endothelin 1 signaling in retinal arteriolar smooth muscle. Invest Ophthalmol Vis Sci 53 (6):3059-66. 
Sun, X. P., N. Callamaras, J. S. Marchant, and I. Parker. 1998. A continuum of InsP3mediated elementary Ca2+ signalling events in Xenopus oocytes. J Physiol 509 ( Pt 1):67-80.

Swillens, S., P. Champeil, L. Combettes, and G. Dupont. 1998. Stochastic simulation of a single inositol 1,4,5-trisphosphate-sensitive $\mathrm{Ca} 2+$ channel reveals repetitive openings during 'blip-like' Ca2+ transients. Cell Calcium 23 (5):291-302.

Syyong, H. T., H. H. Yang, G. Trinh, C. Cheung, K. H. Kuo, and C. van Breemen. 2009. Mechanism of asynchronous $\mathrm{Ca}(2+)$ waves underlying agonist-induced contraction in the rat basilar artery. Br J Pharmacol 156 (4):587-600.

Tasker, P. N., F. Michelangeli, and G. F. Nixon. 1999. Expression and distribution of the type 1 and type 3 inositol 1,4,5-trisphosphate receptor in developing vascular smooth muscle. Circ Res 84 (5):536-42.

Thul, R., and M. Falcke. 2004. Release currents of IP(3) receptor channel clusters and concentration profiles. Biophys $J$ 86 (5):2660-73.

Tovey, S. C., P. de Smet, P. Lipp, D. Thomas, K. W. Young, L. Missiaen, H. De Smedt, J. B. Parys, M. J. Berridge, J. Thuring, A. Holmes, and M. D. Bootman. 2001. Calcium puffs are generic InsP(3)-activated elementary calcium signals and are downregulated by prolonged hormonal stimulation to inhibit cellular calcium responses. J Cell Sci 114 (Pt 22):3979-89.

Tran, C. H., M. S. Taylor, F. Plane, S. Nagaraja, N. M. Tsoukias, V. Solodushko, E. J. Vigmond, T. Furstenhaupt, M. Brigdan, and D. G. Welsh. 2012. Endothelial Ca2+ wavelets and the induction of myoendothelial feedback. Am J Physiol Cell Physiol 302 (8):C1226-42.

Tsoukias, N. M. 2011. Calcium dynamics and signaling in vascular regulation: computational models. Wiley Interdiscip Rev Syst Biol Med 3 (1):93-106.

Tsoukias, N. M., M. Kavdia, and A. S. Popel. 2004. A theoretical model of nitric oxide transport in arterioles: frequency- vs. amplitude-dependent control of cGMP formation. Am J Physiol Heart Circ Physiol 286 (3):H1043-56.

Vane, J. R. 1964. The Use of Isolated Organs for Detecting Active Substances in the Circulating Blood. Br J Pharmacol Chemother 23:360-73.

Wagner, J., and J. Keizer. 1994. Effects of rapid buffers on Ca2+ diffusion and Ca2+ oscillations. Biophys $J 67$ (1):447-56.

Wang, I. Y., Y. Bai, M. J. Sanderson, and J. Sneyd. 2010. A mathematical analysis of agonist- and $\mathrm{KCl}$-induced $\mathrm{Ca}(2+)$ oscillations in mouse airway smooth muscle cells. Biophys $J 98$ (7):1170-81. 
Wang, Q., R. C. Hogg, and W. A. Large. 1992. Properties of spontaneous inward currents recorded in smooth muscle cells isolated from the rabbit portal vein. $J$ Physiol 451:525-37.

Wang, S. Q., L. S. Song, E. G. Lakatta, and H. Cheng. 2001. Ca2+ signalling between single L-type Ca2+ channels and ryanodine receptors in heart cells. Nature 410 (6828):592-6.

Wang, S. S., and G. J. Augustine. 1995. Confocal imaging and local photolysis of caged compounds: dual probes of synaptic function. Neuron 15 (4):755-60.

Woods, N. M., K. S. Cuthbertson, and P. H. Cobbold. 1986. Repetitive transient rises in cytoplasmic free calcium in hormone-stimulated hepatocytes. Nature 319 (6054):600-2.

Yashiro, Y., and B. R. Duling. 2000. Integrated $\mathrm{Ca}(2+)$ signaling between smooth muscle and endothelium of resistance vessels. Circ Res 87 (11):1048-54.

Zang, W. J., J. Zacharia, C. Lamont, and W. G. Wier. 2006. Sympathetically evoked Ca2+ signaling in arterial smooth muscle. Acta Pharmacol Sin 27 (12):1515-25.

Zeng, S., B. Li, and S. Chen. 2009. Simulation of spontaneous Ca2+ oscillations in astrocytes mediated by voltage-gated calcium channels. Biophys $J 97$ (9):2429-37.

Zheng, Y. M., Q. S. Wang, Q. H. Liu, R. Rathore, V. Yadav, and Y. X. Wang. 2008. Heterogeneous gene expression and functional activity of ryanodine receptors in resistance and conduit pulmonary as well as mesenteric artery smooth muscle cells. J Vasc Res 45 (6):469-79. 


\section{CHAPTER 2- LOCALIZED CALCIUM EVENTS ARISING FROM OPENING OF CLUSTER OF TRPV4 CHANNELS}

This chapter is accepted in Biophysical Journal (in press) as Parikh, J., A. Kapela and N. M.Tsoukias. "Stochastic model of a TRPV4 Calcium sparklet: Effect of bursting and cooperativity on endothelial-derived hyperpolarization" 


\section{$2.1 \quad$ Abstract}

We examined the endothelial transient receptor vanilloid 4 (TRPV4) channel vasodilatory signaling in the regulation of vessel tone using mathematical modeling. The model analyzes experimental data by Sonkusare et al. (Science 336: 597-601, 2012) on TRPV4-induced endothelial $\mathrm{Ca}^{2+}$ events (sparklets). A previously developed continuum model of an endothelial and a smooth muscle cell coupled through microprojections (MPs), was extended to account for the activity of a TRPV4 channel cluster. Different stochastic descriptions for the TRPV4 channel flux were examined using finite state Markov chains. The model also took into consideration recent evidence for the colocalization of intermediate-conductance calcium-activated potassium channels $\left(\mathrm{IK}_{\mathrm{Ca}}\right)$ and TRPV4 channels near the MPs. A single TRPV4 channel opening resulted in a stochastic localized $\mathrm{Ca}^{2+}$ increase in a small region (i.e. few $\mu \mathrm{m}^{2}$ area) close to the channel. We predict micromolar $\mathrm{Ca}^{2+}$ increases lasting for the open duration of the channel, sufficient for the activation of low-affinity endothelial $\mathrm{K}_{\mathrm{Ca}}$ channels. Simulations of a cluster of four TRPV4 channels incorporating burst and cooperative gating kinetics, provided quantal $\mathrm{Ca}^{2+}$ increases (i.e. steps of fixed amplitude), similar to the experimentally observed $\mathrm{Ca}^{2+}$ sparklets. These localized $\mathrm{Ca}^{2+}$ events result in endothelium-derived hyperpolarization (EDH) (and SMC relaxation) with magnitude that depends on event frequency. The gating characteristics (bursting, cooperativity) of the TRPV4 cluster enhance $\mathrm{Ca}^{2+}$ spread and the distance of $\mathrm{K}_{\mathrm{Ca}}$ channel activation. This may amplify the EDH response by the additional recruitment of distant $\mathrm{K}_{\mathrm{Ca}}$ channels. 


\subsection{Introduction}

A complex bidirectional communication between endothelial (EC) and smooth muscle (SMC) cells regulates SMC constriction and vessel tone. Cytosolic calcium $\left(\mathrm{Ca}^{2+}\right)$ regulates the ability of ECs to induce the release of vasoactive signals including the discharge of hyperpolarizing current to the SMC. In small resistance vessels, this NOindependent endothelium-derived hyperpolarizing $(\mathrm{EDH})$ signaling is typically mediated by the activation of intermediate- $\left(\mathrm{IK}_{\mathrm{Ca}}\right)$ and small- $\left(\mathrm{SK}_{\mathrm{Ca}}\right)$ conductance $\mathrm{Ca}^{2+}$-activated potassium channels in response to an increase in $\mathrm{Ca}^{2+}$ concentration (Edwards, Feletou, and Weston 2010; Garland, Hiley, and Dora 2011). Global EC Ca ${ }^{2+}$ mobilization results from transmembrane $\mathrm{Ca}^{2+}$ influx and/or $\mathrm{Ca}^{2+}$ release from the intracellular stores. Recent evidence suggest that localized $\mathrm{Ca}^{2+}$ influx from spontaneous or agonist-induced opening of the transient receptor vanilloid 4 (TRPV4) channel can also induce SMC hyperpolarization and vessel dilation through the EDH mechanism in resistance vessels (Bagher et al. 2012; Sonkusare et al. 2012; Sonkusare et al. 2014).

TRPV4 channels are sensitive to a wide array of stimuli including, Epoxyeicosatrienoic acids (EETs), Diacylglycerold and phorbol esters via PKC dependent and independent pathways, osmotic changes, mechanical stimuli, $\mathrm{Ca}^{2+}$ levels and temperature (Heller and O'Neil 2007; Strotmann, Schultz, and Plant 2003; Nilius et al. 2004). In the vasculature, EC agonists like acetylcholine (Sonkusare et al. 2012; Sonkusare et al. 2014; Earley et al. 2009), shear stress (Mendoza et al. 2010) and sustained low pressures (Bagher et al. 2012), in addition to exogenous agonist like GSK1016790A (GSK), 4- $\alpha$ phorbol 12,13-didecanoate (4aPDD) or 11-12 EET, may activate TRPV4 channels to produce a transient stationary $\mathrm{Ca}^{2+}$ burst (sparklet) within the 
EC that may ultimately result in vasodilation. Single channel patch clamp data reveals stochastic TRPV4 opening with pA current amplitude at resting membrane potential (-50 mv) (Watanabe et al. 2002). Activation of as few as three channels per EC may be sufficient to induce maximal vessel dilation (Sonkusare et al. 2012). The role of individual activators and pathways resulting in TRPV4 channel openings is currently under investigation. Recent evidence suggests bursting activity and cooperative opening of TRPV4 channels in a cluster (Sonkusare et al. 2012). The physiological relevance of such gating mechanisms has not been elucidated.

EC extensions over the internal elastic lamina towards SMCs, termed myoendothelial projections (MPs), have been observed in small diameter vessels (Sandow et al. 2009; Heberlein, Straub, and Isakson 2009) and can play a role in the modulation of vascular tone (Kerr et al. 2012). Recent studies provide evidence for the localization of TRPV4 channels with $\mathrm{IK}_{\mathrm{Ca}}$, inositol 1,4,5-triphosphate receptors ( $\left.\mathrm{IP}_{3} \mathrm{Rs}\right)$, and connexins in/near MPs (Bagher et al. 2012; Sandow et al. 2006; Ledoux et al. 2008; Isakson 2008; Dora et al. 2008). Myoendothelial gap junctions (MEGJs) are often found at the tip of the MP, and allow for electrochemical communication between ECs and SMCs (Heberlein, Straub, and Isakson 2009; Sandow et al. 2009). Spontaneous and agonist-induced localized $\mathrm{Ca}^{2+}$ events mediated by transmembrane $\mathrm{Ca}^{2+}$ influx (TRPV4 sparklets (Bagher et al. 2012; Sonkusare et al. 2012; Sonkusare et al. 2014)) or intracellular store $\mathrm{Ca}^{2+}$ release ('pulsars'(Ledoux et al. 2008) and 'wavelets'(Tran et al. 2012)) have been observed in ECs near the MP sites. 
Mathematical modeling offers a systematic approach for the analysis of complex signaling mechanisms, and it can serve as a tool for data interpretation and for guiding new experimental studies. Few theoretical studies have been carried out to investigate the role of ECs and MPs in the modulation of SMC Ca ${ }^{2+}$ and membrane potential $\left(V_{\mathrm{m}}\right)$ dynamics. We have previously examined SMC $\mathrm{Ca}^{2+}$ and $V_{\mathrm{m}}$ changes during EC stimulation through the development of an EC-SMC compartmental model (Kapela, Bezerianos, and Tsoukias 2009). This model integrates detailed single cell EC (Silva, Kapela, and Tsoukias 2007) and SMC (Kapela, Bezerianos, and Tsoukias 2008) models with electrical, chemical and NO coupling pathways. Acetylcholine (Ach) stimulation of ECs in the model increased global $\mathrm{EC} \mathrm{Ca}^{2+}$ levels, activated EDH and NO pathways to hyperpolarize the SMC which ultimately reduced global $\mathrm{Ca}^{2+}$ concentration in the SMC. We have also extended the compartmental model into a 2D continuum model which incorporates accurate MP geometry from electron microscopy images and spatial localization of $\mathrm{IK}_{\mathrm{Ca}}$ and $\mathrm{IP}_{3} \mathrm{Rs}$ in the MP. This new formulation was utilized to investigate the role of feedback in EC-SMC communication (Nagaraja et al. 2013). Similarly, Brasen et al. have developed a 2D-axisymmetric model incorporating the anatomical structure of MPs into a two cell system (Brasen, Jacobsen, and HolsteinRathlou 2012). Their results show that MPs may rectify the signal between the EC and SMC. Previous models did not examine the role of TRPV4 channels. Moreover, they considered deterministic whole cell current descriptions for membrane channels and pumps, and did not account for localized and stochastic channel openings. 
In this study, we present the development of a computational model to examine the localized $\mathrm{Ca}^{2+}$ mobilization, in the vicinity of the MP, arising from a single or a cluster of TRPV4 channels. The TRPV4s were incorporated in a previously developed continuum EC-SMC model with MPs. The model accounts for preferential presence of the TRPV4s near the MPs as suggested in experimental studies. Stochastic opening of a TRPV4 channel was captured using a finite state Markov chain. We utilize this model to examine the contribution of these channels to the regulation of vessel tone.

\subsection{Methods}

\subsubsection{Continuum model}

We have presented a general computational framework for modeling spatiotemporal $\mathrm{Ca}^{2+}$ events integrated with plasma membrane electrophysiology in single or coupled vascular cells in (Nagaraja et al. 2013; Kapela and Tsoukias 2011). The model assumes EC and SMC as simplified rectangular domains with dimensions as shown in Figure. 2.1A and implements only half of the EC and SMC by assuming symmetry for the other half. Moreover, the model incorporates an accurate MP geometry from experimental images and assumed high density of $\mathrm{IK}_{\mathrm{Ca}}(25 \%$ of total, under control conditions) and $\mathrm{IP}_{3} \mathrm{Rs}(10 \%$ of total $)$ within the MP. The continuum model takes into account concentration gradients of $\mathrm{Ca}^{2+}$ and other ions within the EC and MP. The transport for individual ionic species is influenced by both electrical and concentration gradients, and was described using the Nernst-Planck electrodiffusion equation (Equation. 2.1)

$$
\delta_{b u f f} \frac{\partial[S]}{\partial t}=\nabla \cdot\left(D_{S} \nabla[S]+z_{s} F u_{m s}[S] \nabla V\right)-R_{s}
$$

Equation. 2.1 
where $S=\mathrm{Na}^{+}, \mathrm{K}^{+}, \mathrm{Cl}^{-}, \mathrm{Ca}^{2+}, D_{S}$ is the diffusion coefficient of ionic species $S, z_{S}$ is the valence of ionic species $S, \nabla V$ is the electrical gradient, $F$ is the Faraday constant and $u_{m S}$ is the ionic mobility given by $\frac{D_{S}}{R T}\left(\mathrm{R}\right.$ is the ideal gas constant $\left(8341 \mathrm{~mJ} \cdot \mathrm{mol}^{-1} \cdot \mathrm{K}^{-1}\right)$ and $\mathrm{T}$ is the absolute temperature). $R_{\mathrm{s}}$ is the source/sink term which includes the expressions for cytosolic $\mathrm{Ca}^{2+}$ exchange with the $\mathrm{ER} / \mathrm{SR}$, and $\mathrm{Ca}^{2+}$ buffering in the $\mathrm{EC}$ and MP. $\delta_{\text {buff }}$ accounts for the $\mathrm{Ca}^{2+}$ buffering in the SMC using a fast buffering approximation. Transport of cytosolic $\mathrm{IP}_{3}$ and $\mathrm{Ca}^{2+}$ within a uniformly distributed ER/SR store was described using Equation. 2.2.

$$
\delta_{b u f f} \frac{\partial[S]}{\partial t}=\nabla .\left(D_{S} \nabla[S]\right)-R_{s}
$$

Equation. 2.2

where $[S]$ is the concentration of either species $\left(\mathrm{IP}_{3}, \mathrm{Ca}^{2+} \mathrm{ER}\right.$ and $\left.\mathrm{SR}\right)$ in the store. $R_{S}$ is the source/sink term and includes $\mathrm{Ca}^{2+}$ exchange between stores and the cellular domains, and $\mathrm{IP}_{3}$ production and degradation. A uniform distribution of transmembrane channels and pumps was considered along the boundary of the cellular domains. The membrane currents were defined as boundary fluxes (Equation. 2.3) across the top and bottom boundaries of EC, SMC and the MP boundaries.

$$
-n N_{S}=\frac{1}{z_{S} F} \sum_{K} I_{S, K}
$$

Equation. 2.3

where $n$ is the normal to the surface and $N_{s}$ is the membrane flux given by summation of all the transmembrane currents species for species $\mathrm{S}\left(I_{S, K}\right)$. The membrane currents were distributed between the MP and bulk cell according to their respective volumes. The 
membrane current definitions and parameters are identical to the original models (Kapela, Bezerianos, and Tsoukias 2008, 2009; Kapela and Tsoukias 2011; Silva, Kapela, and Tsoukias 2007).

To estimate the characteristics of the TRPV4 mediated $\mathrm{Ca}^{2+}$ event in the EC and resulting vessel tone modulation, the model in Nagraja et al. (Nagaraja et al. 2013) was modified through the introduction of the single or a cluster of four TRPV4 channels in a localized region on the EC boundary $0.5 \mu \mathrm{m}$ away from the MP (Figure. 2.1-B). The current carried by each cation S $\left(I_{T R P V 4, S}\right)$ through a single TRPV4 channel is described using Equation. 2.4.

$$
I_{T R P V 4, S}\left(t, V_{m}, S_{i}\right)=P_{T R P V 4, S} \frac{z_{s}^{2} F^{2}}{R T} V_{m} \frac{S_{i}-S_{0} e^{\frac{-z_{s} V_{m} F}{R T}}}{1-e^{\frac{-z_{s} V_{m} F}{R T}}}
$$

Equation. 2.4

where $\mathrm{S}_{\mathrm{i}}$ is the concentration of $\mathrm{Ca}^{2+}, \mathrm{K}^{+}, \mathrm{Na}^{+}$inside the $\mathrm{EC}, \mathrm{S}_{\mathrm{o}}$ is the extracellular concentration, $\mathrm{F}$ is the Faradays constant, $P_{T R P V 4, S}$ is the ionic permeability and $V_{m}$ is the membrane potential.

The net current $\left(I_{T R P V 4, \text { total }}\right)$ through the channel (Equation. 2.5) modulates the membrane potential $\left(V_{m}\right)$ described using standard Hodgkin Huxley formalism (Silva, Kapela, and Tsoukias 2007).

$$
I_{T R P V 4, \text { total }}=\sum I_{T R P V 4, S}
$$

Equation. 2.5 
A)

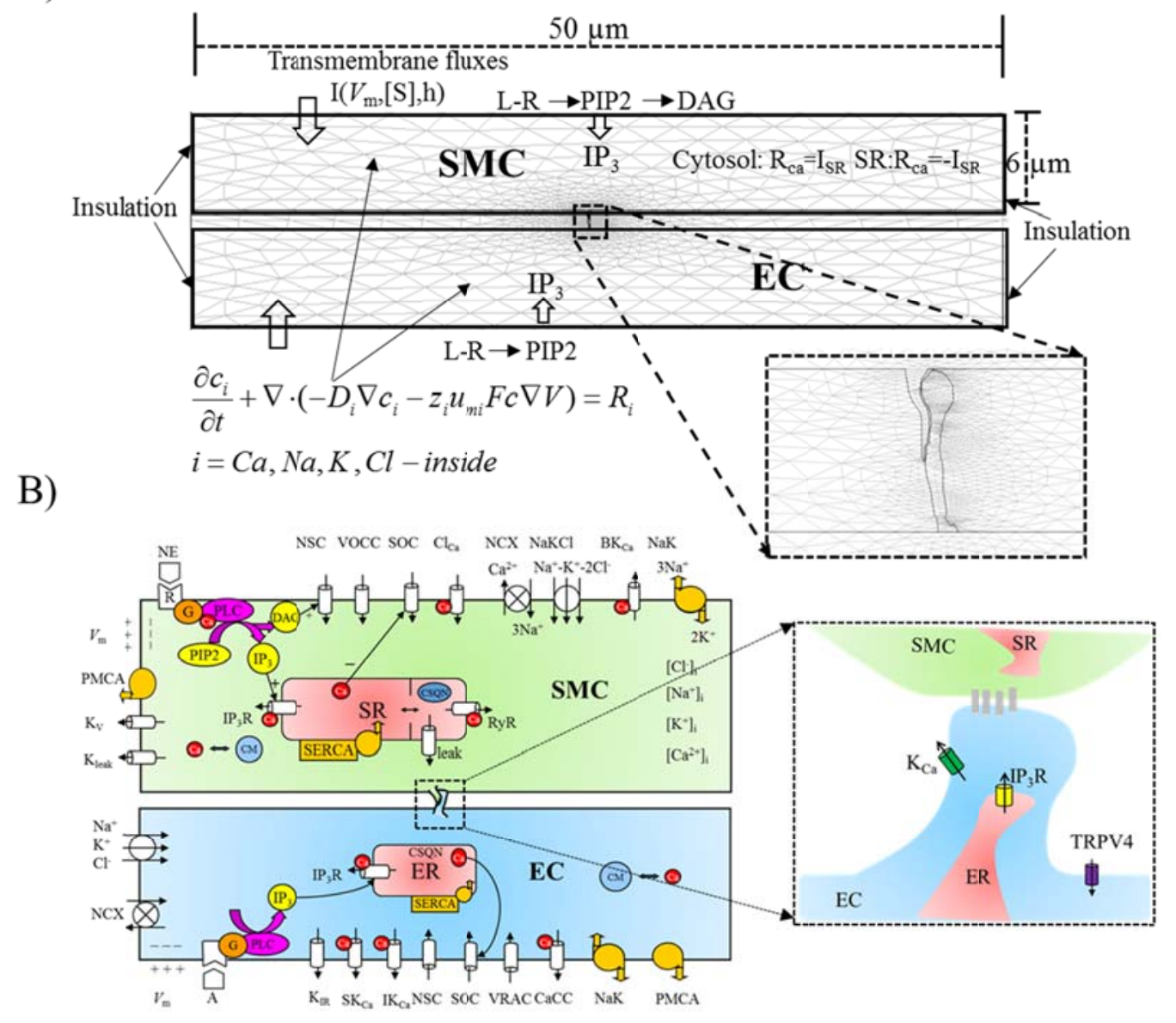

Figure 2.1 Schematic of the continuum EC-SMC model, (A) 2D axisymmetric model geometry with SMC and EC as rectangular domains coupled with EC MP and MEGJs (B) Cartoon illustration describing all the channels and pumps incorporated in the EC-MP-SMC continuum model in (A).

A single TRPV4 channel-conductance of $45 \mathrm{pS}$ for inward currents (at -100 to $0 \mathrm{mV}$ ) was reported using inside-out patch clamp under symmetric intra- and extra- cellular ionic concentrations (Loukin et al. 2010), and a single channel-conductance of 56 to 66 $\mathrm{pS}$ for inward currents (at -100 to $0 \mathrm{mV}$ ) were observed under near physiological ionic concentrations using cell-attached patch clamp technique (Watanabe et al. 2002). We estimated the permeability for each ion $\left(P_{T R P V 4, S}\right)$ to match these conductance's, using the $I_{T R P V 4, \text { total }}$ description (Eqs. $\left.4 \& 5\right)$ and reported permeability ratios $\left(P_{T R P V 4, C a}: P_{T R P V 4, K}:\right.$ $P_{\text {TRPV } 4, \mathrm{Na}}=7.1: 1.42: 1$ (Ma et al. 2011)). The calculated $\mathrm{Ca}^{2+}$ permeability for a single 
TRPV4 channel $P_{T R P V 4, \mathrm{Ca}}$ was $6.8 \times 10^{-13}-8.5 \times 10^{-13} \mathrm{~cm}^{3} / \mathrm{s}$ (which corresponds $4 \times 10^{-8}-$ $5 \times 10^{-8} \mathrm{~cm} / \mathrm{s}$ assuming $1 \mu \mathrm{F} / \mathrm{cm}^{2}$ standard membrane capacitance and total EC membrane capacitance of $17 \mathrm{pF}$ ). In the model we use $P_{T R P V 4, C a}$ control value of $4.5 \times 10^{-8} \mathrm{~cm} / \mathrm{s}$. This corresponds to control values for $P_{T R P V, N a}$ and $P_{T R V 4, K}$ of $6.5 \times 10^{-9} \mathrm{~cm} / \mathrm{s}$ and $8.77 \times 10^{-9}$ $\mathrm{cm} / \mathrm{s}$ respectively.

\subsubsection{Stochastic opening of TRPV4 channel}

\subsubsection{Two-state model}

Individual channels are not constantly open and the ionic currents fluctuate stochastically. A single channel can be represented using a continuous-time homogenous finite state Markov chain, which describes reversible transitions between a finite number of distinct states in which the ion channel can reside (Colquhoun and Hawkes 1982; Ball and Rice 1992). The probability of transitions from one state to another is assumed to be independent of the current state. As a simplest case, we considered the TRPV4 channel to be either in conducting (open) state or non-conducting (close) state (Mechanism. 1)

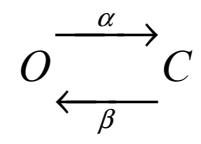

Mechanism 1

where $\mathrm{O}$ is the open state, $\mathrm{C}$ the close state and $\alpha, \beta$ define the rate of transition from one state to another. The probability density function (pdf) for the closed-channel lifetime $\left(f_{c}\right)$ and the open-channel lifetime $\left(f_{o}\right)$ are distributed exponentially (Equation. 2.6).

$$
\begin{aligned}
& f_{c}=\beta e^{-\beta t} \\
& f_{o}=\alpha e^{-\alpha t}
\end{aligned}
$$

Equation. 2.6 
The mean life time in any state is given by the reciprocal of sum of the transition rates that lead away from the state (Colquhoun and Hawkes 1981). This provides a mean open lifetime and mean closed lifetime of $1 / \alpha$ and $1 / \beta$ respectively for the two-state model in Mechanism 1. The transition rates $\alpha, \beta$ were calculated based on the reported mean open time (37ms; 1/ $\alpha$ ) in Sonkusare et al. (Sonkusare et al. 2012) and an assumed mean closed time of $780 \mathrm{~ms}(1 / \beta)$. This estimate for the mean closed time provides an open probability $\left(P_{o} ; /(\alpha+\beta)\right)$ for an individual two-state TRPV4 channel of 0.045 as observed in (Sonkusare et al. 2012).

\subsubsection{Three-state model}

$\mathrm{Ca}^{2+}$ data obtained from a cluster of TRPV4 channels (Bagher et al. 2012; Sonkusare et al. 2012) reveals a $\mathrm{Ca}^{2+}$ burst of few seconds followed by longer inactivity periods, suggesting burst kinetics (i.e. openings are separated by short closed periods, before a long closed period). The two-state model cannot capture the experimentally observed $\mathrm{Ca}^{2+}$ burst. We simulated stochastic opening of a single TRPV4 channel using a simple three-state model (Colquhoun and Hawkes 1982) as given in Mechanism 2.

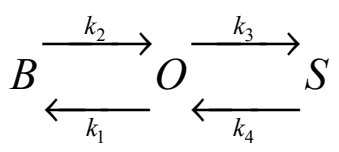

Mechanism 2

where $\mathrm{B}$ is the block state (intraburst short closed state), $\mathrm{O}$ is the open state and $\mathrm{S}$ is the shut state (interburst long closed state). $\mathrm{k}_{1}, \mathrm{k}_{2}, \mathrm{k}_{3}$ and $\mathrm{k}_{4}$ define the rates of transition from one state to another. The TRPV4 channel can reside in either of these three states. The model in Mechanism 2 results in mean open time, mean blocked time, mean shut time and burst length given by $1 /\left(\mathrm{k}_{3}+\mathrm{k}_{1}\right), 1 / \mathrm{k}_{2}, 1 / \mathrm{k}_{4}$ and $\left(1+\mathrm{k}_{1} / \mathrm{k}_{2}\right) / \mathrm{k}_{3}$ respectively. The 
transition rates $\mathrm{k}_{1}$ to $\mathrm{k}_{4}$ were calculated based on the reported mean open time (37ms) of the TRPV4 channel, and observed long inactivity (shut time) of about 1 min and burst duration of around $5 \mathrm{sec}$ from the $\mathrm{Ca}^{2+}$ data in Sonkusare et al. (Sonkusare et al. 2012). We assumed a mean channel blocked time $(\sim 33 \mathrm{~ms})$ to obtain an overall channel $\mathrm{P}_{\mathrm{o}}$ of 0.045 as in the two-state model. The overall probability $\mathrm{P}_{0}$ was calculated as described in (Colquhoun and Hawkes 1982). The three-state model provides a significantly higher open probability during the burst $\left(\mathrm{P}_{\mathrm{o}}^{\text {burst }}\right)$ of about 0.5 .

\subsubsection{Cluster of independent or cooperative TRPV4 channels}

Stochastic opening of a cluster of four independent TRPV4 channels, with each individual channel represented by the Markov two-state and three-state chain described in Mechanism 1 and Mechanism 2 respectively, were simulated using a reduced transition rate matrix (see supplement section 2.8). Given a group of $\mathrm{N}$ independent and identical TRPV4 channels, the probability that $\mathrm{k}$ of these channels are open, $\mathrm{p}(\mathrm{k})$, is described by the binomial distribution (Equation. 2.7).

$$
p(k)=\left(\begin{array}{l}
N \\
k
\end{array}\right) P_{o}^{k}\left(1-P_{o}\right)^{N-k}
$$

Equation. 2.7

A two minute long simulation of a cluster of four independent channels followed the binomial distribution (see results). The $\mathrm{P}_{\mathrm{o}}$ of each individual channel in a cluster of four independent channels was observed to be 0.045 as before, using both the two- and threestate models. The average number of open (active) TRPV4 channels $\left(\mathrm{NP}_{\mathrm{o}}\right)$ in this simulation was 0.17 (close to the theoretical value of $4 * \mathrm{P}_{\mathrm{o}}$ of 0.18 ). 
Data in (Sonkusare et al. 2012) suggest that opening of two, three or four TRPV4 channels are significantly more frequent than expected based on the binomial distribution, indicating an interaction between the channels within a cluster. The cooperative two- and three-state models account for these channel interactions in a cluster through modulation of the transition rate from closed to open state $(\beta)$, and shut state to open state $\left(\mathrm{k}_{4}\right)$ respectively, when at least one other channel in the cluster is in the open state (see supplement). For example, as shown in Figure.2.2 for a cluster of four channels described using the three-state model, if the channel 3 is in open state, the transition rate $\mathrm{k}_{4}$ for the channel 1,2 and 4 was increased to $\mathrm{k}_{4}{ }^{\prime}$ to allow for cooperativity between the channels. Cooperativity increased average number of open (active) TRPV4 channels $\left(\mathrm{NP}_{\mathrm{o}}\right)$ to 0.27 in both the two- and three-state models.

A)

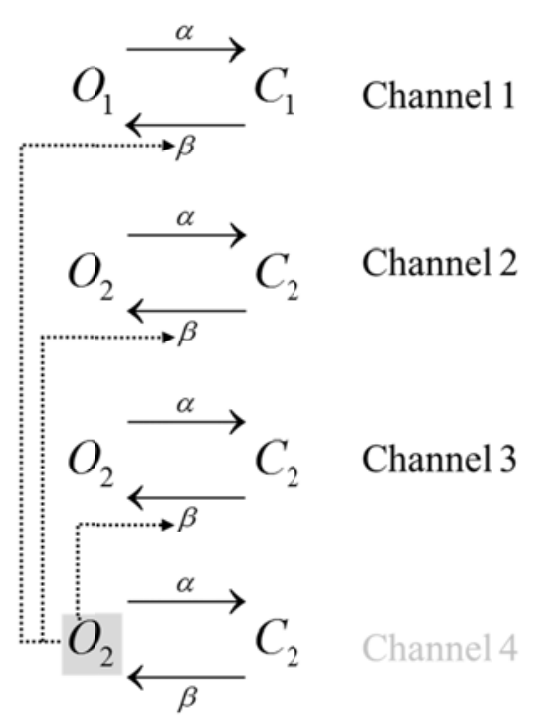

B)

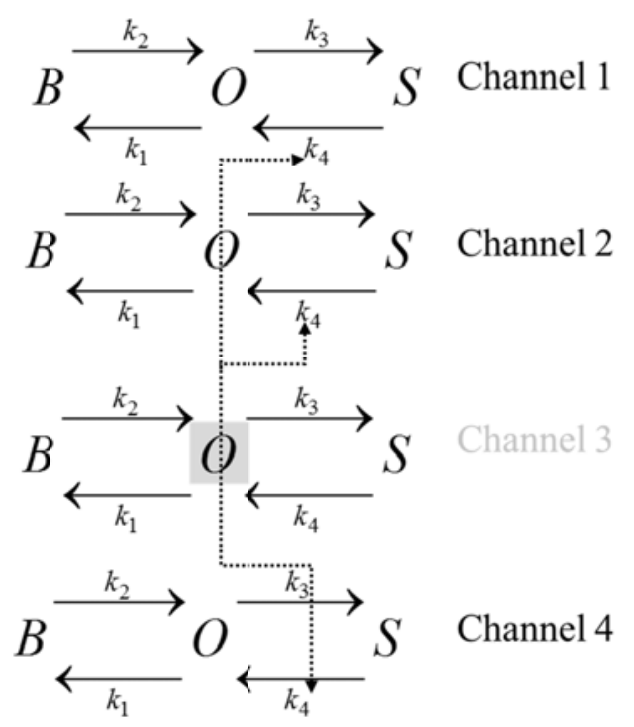

Figure 2.2 Cooperativity implementation of four TRPV4 channels, (A) Four TRPV4 channel in a cluster described using a simple two-state (open and close) Markov chain model. The rate parameter $\beta$, for transition of a channel from the closed state to the open state, was increased in the presence of at least one other channel in the open state. (B) TRPV4 channels implemented using a three-state (shut, block and open) Markov chain to capture burst opening of the channel. The rate parameter $\mathrm{k}_{4}$ describing the transition of a channel from the shut state to the open state was increased in the presence of at least one other channel in the open state. 


\subsection{Results}

\subsubsection{Continuous TRPV4 opening}

A single TRPV4 channel opening was simulated through the introduction of TRPV4 current (Equation. 2.5). $\mathrm{Ca}^{2+}$ levels arising from continuous opening of the TRPV4 channel for 10,100, 200, and $4000 \mathrm{~ms}$ are depicted color-coded in Figure. 2.3-A. A micromolar increase in $\mathrm{Ca}^{2+}$ levels was observed within few milliseconds in a small area $\left(0.25 \mu \mathrm{m}^{2}\right)$ around the TRPV4 channel location. Channel opening for longer durations, increases the spread area of the $\mathrm{Ca}^{2+}$ event. The resulting $\mathrm{Ca}^{2+}$ spread can activate $\mathrm{Ca}^{2+}-$ activated potassium channels $\left(\mathrm{K}_{\mathrm{Ca}}\right)$ in the vicinity of the TRPV4 location. The contour lines in Figure. 2.3-B (black, yellow, red and brown) represent the region of $\mathrm{Ca}^{2+}$ spread for 10,100, 200 and $4000 \mathrm{~ms}$ TRPV4 open duration respectively. The region was defined by $\mathrm{Ca}^{2+}$ concentrations higher than the half activation of the $\mathrm{IK}_{\mathrm{Ca}}$ channel $\left[\left(\mathrm{EC}_{50}\right) 740 \mathrm{nM}\right.$ (Ahn et al. 2004)]. Simulations predict $\mathrm{Ca}^{2+}$ activity spreading up to 0.3-6 $\mu \mathrm{m}$ radial distances for 10-4000 ms open time durations. The increase in the $\mathrm{Ca}^{2+}$ spread area did not increase linearly with channel open time.

\subsubsection{Stochastic TRPV4 opening}

Stochastic opening of a TRPV4 channel represented by a simple two-state Markov model (Mechanism 1) was simulated using a transition rate matrix (Equation. 2.11 in section 2.8) with $\alpha=2.7 \times 10^{-2} \mathrm{~ms}^{-1}$ and $\beta=1.3 \times 10^{-3} \mathrm{~ms}^{-1}$. A representative single channel record of 120 second duration is shown in supplement (Figure. 2.9-A), displaying random opening and closing of the TRPV4 channel with an overall $\mathrm{P}_{\mathrm{o}}$ of 0.045 . Simulations with two-state model could not capture the long quiescent period of TRPV4 channel inactivity observed in the experiment (Sonkusare et al. 2012). 


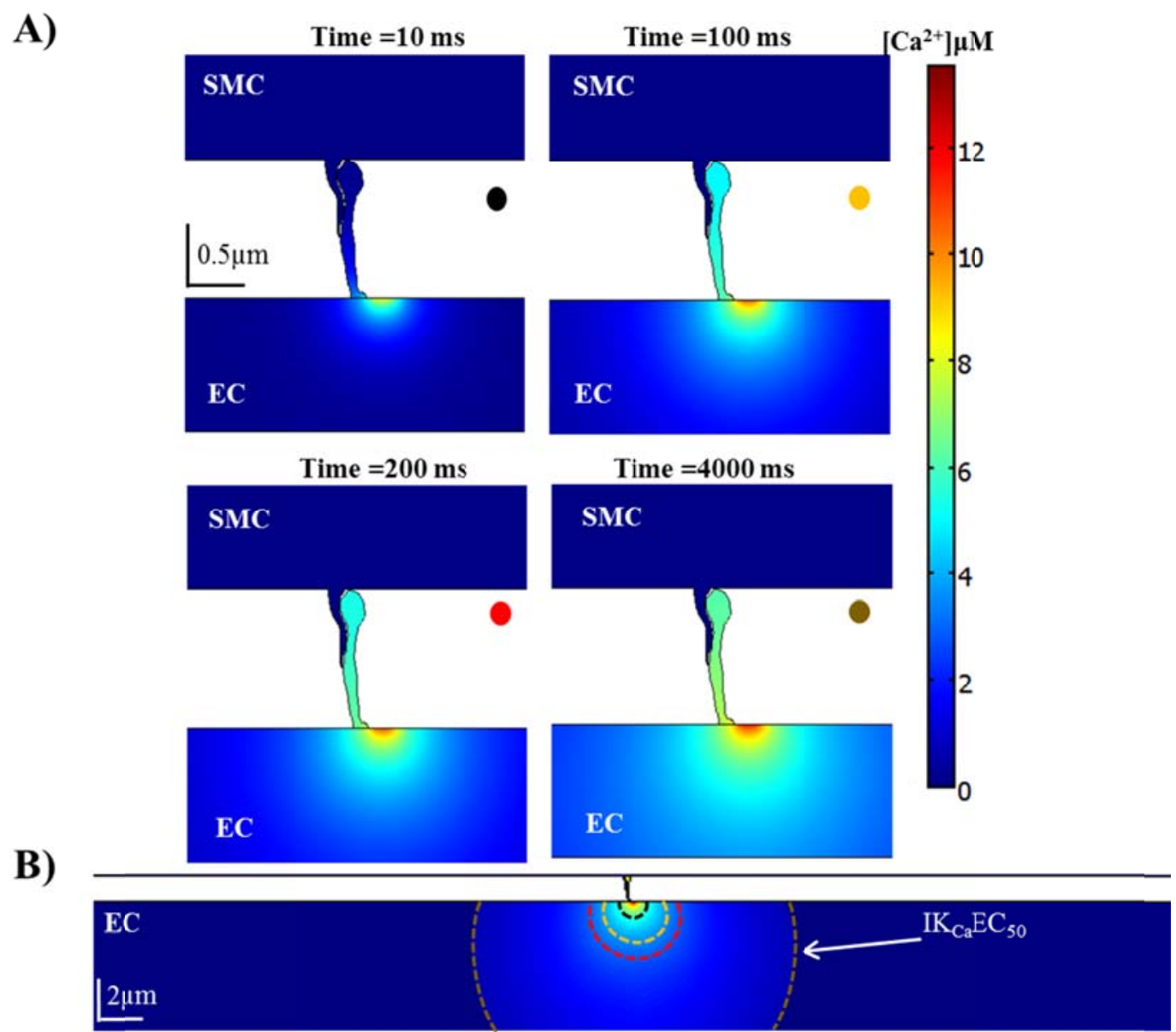

Figure 2.3 Spatial $\mathrm{Ca}^{2+}$ profiles resulting from a single TRPV4 channel opening. (A) Continuous opening of the TRPV4 channel for open times of 10, 100, 200 and $4000 \mathrm{~ms}$ resulted in $\mu \mathrm{M} \mathrm{Ca}^{2+}$ concentrations progressively spreading over a larger area. (B) Contour lines $\left(\mathrm{Ca}^{2+}\right.$ concentration equal to $\mathrm{EC}_{50}$ of $\left.\mathrm{IK}_{\mathrm{Ca}}\right)$ indicate increasing $\mathrm{Ca}^{2+}$ spatial spread for $10,100,200$ and $4000 \mathrm{~ms}$ of continuous opening of a TRPV4 channel and highlight the time evolution of the cell regions with at least $50 \% \mathrm{IK}_{\mathrm{Ca}}$ activity.

\subsubsection{Three-state model}

Three-state Markov chain model (Mechanism 2) was used to describe a single TRPV4 channel exhibiting bursting activity. Simulations were performed using a transition rate matrix obtained from the three-state model with $\mathrm{k}_{1}=2.35 \times 10^{-2} \mathrm{~ms}^{-1}, \mathrm{k}_{2}=4.35 \times 10^{-2} \mathrm{~ms}^{-1}$, $\mathrm{k}_{3}=3.23 \times 10^{-4} \mathrm{~ms}^{-1}$ and $\mathrm{k}_{4}=1.69 \times 10^{-5} \mathrm{~ms}^{-1}$ (Equation. 2.12 in section 2.8). These values were derived from the observed open time, open probability, interburst interval and burst duration in Sonkusare et al (Sonkusare et al. 2012).as described in the methods section. A 
representative single channel record with burst openings is shown in Figure.2.4-A.

TRPV4 superposition records for a cluster of four independent channels were simulated

(data not shown) using the reduced transition rate matrix of the three-state model

(Equation. 2.15 in section 2.8). Comparison between a single and a cluster of TRPV4

channels enable us to examine the physiological relevance of channel clustering in mediating $\mathrm{Ca}^{2+}$ signaling and $\mathrm{EDH}$.

A)

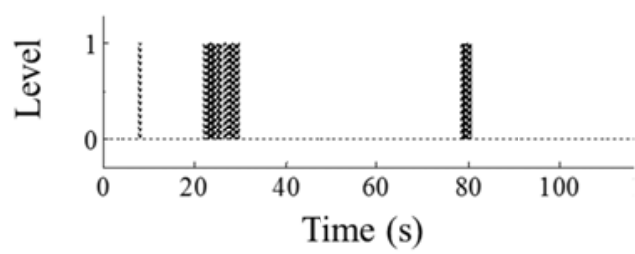

C)

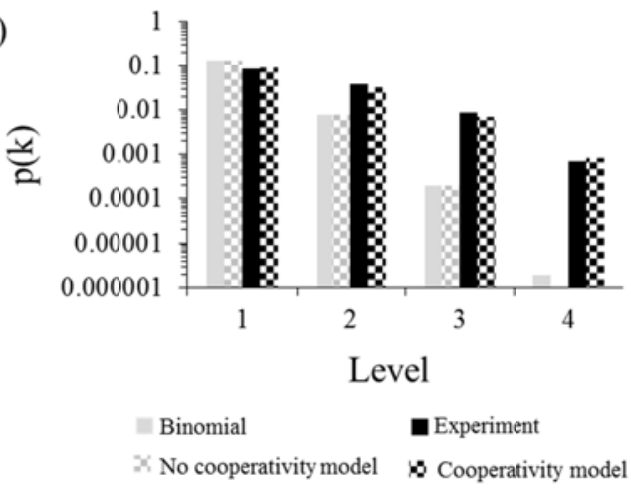

B) Four channel cluster cooperative opening

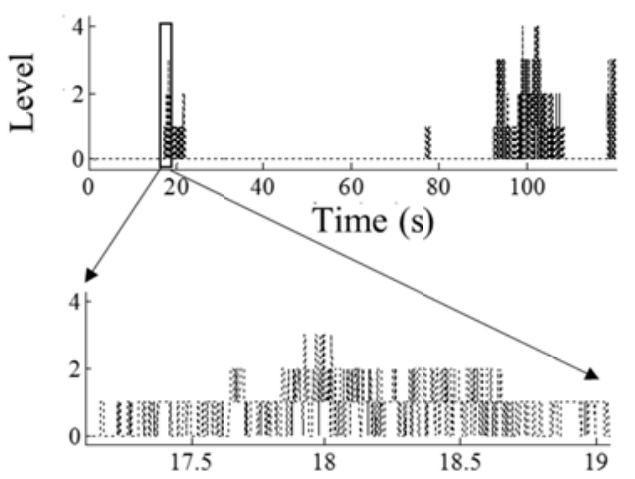

Figure 2.4 Stochastic opening of a cluster of four TRPV4 channels implemented using a three-state Markov chain to simulate burst opening of the channel, (A) Illustrative example of a temporal profile of a single TRPV4 channel transition between the conducting (open) and non-conducting states (shut, block). (B) Example of a superposition temporal profile of the TRPV4 cluster, with the level number describing the number of open channels at a given time. Insert shows zoomed in view of a segment of the total simulation time for better visualization.(C) Experiment (Sonkusare et al. 2012) (black solid bar) and cooperative channel gating in the Markov model (black checkered) demonstrated increased open probabilities of second, third, and fourth channel openings respectively relative to the binomial distribution (grey solid) and a Markov model (grey checkered) considering independent channels.

Cooperativity was implemented by increasing the transition rate $\mathrm{k}_{4}$ to $\mathrm{k}_{4}{ }^{\prime}$ when at least one other channel is in the open state. An 8-fold increase in $\mathrm{k}_{4}{ }^{\prime}$ was considered to match the experimentally observed $\mathrm{NP}_{\mathrm{o}}$ value (Figure. 2.8, in section 2.8). An illustrative 
superposition record of a cooperative TRPV4 cluster is displayed in Figure.2.4-B. A significant number of $2^{\text {nd }}, 3^{\text {rd }}$ and $4^{\text {th }}$ level opening is observed during the burst period (compare with Figure. 2.9-B) followed by a long period of channel idleness. Similar to the two-state model, a cluster of independent channels resulted in an open probability distribution (Figure. 2.4-C, grey checkered bar) which follows a binomial distribution (Figure. 2.4-C, grey solid bar). An increase in the probabilities for second, third and fourth level openings was observed for simulations with cooperativity (Figure. 2.4-C, black checkered bar), in agreement with the experimental observation (Figure. 2.4-C, black solid bar). Statistical chi-squared test described in (Draber, Schultze, and Hansen 1993) was carried out to confirm for cooperativity in the simulations. The three-state model with cooperativity is able to closely capture the salient features of the TRPV4 cluster from the experiments. The different model implementations for the TRPV4 cluster allow us to examine the role of bursting and cooperativity in the modulation of vessel tone.

\subsection{3 $\mathrm{Ca}^{2+}$ and $\mathrm{V}_{\mathrm{m}}$ dynamics: stochastic continuum model}

In the absence of agonist stimulation (i.e. no $\mathrm{Ca}^{2+}$ release from the stores), we examined the $\mathrm{Ca}^{2+}$ and $V_{\mathrm{m}}$ changes induced from opening of a cluster of EC TRPV4s in the continuum model. Stochastic current $\left(I_{\text {stochastic,TRPV4,S }}\right)$ from the TRPV4 channel cluster is provided by Equation. 2.8.

$$
I_{\text {stochastic,TRPV } 4, S}(t)=I_{T R P V 4, S}(t) * N(t)
$$

Equation. 2.8 
where $I_{T R P V 4, S}(t)$ is the TRPV4 flux as described by the GHK equation (Equation. 2.5) and $N(t)$ is the number of open TRPV4 channels at time $t$ given by superposition records such as the records in Figure. 2.4-B and 2.5-B.

A two minute long representative simulation using the three-state cooperative model is displayed in Figure. 2.5. The stochastic TRPV4 openings (Figure. 2.5-A) and the average $\mathrm{Ca}^{2+}$ concentration in a small area $\left(0.25 \mu \mathrm{m}^{2}\right)$ around the cluster's location (Figure. 2.5B) are depicted. The TRPV4 current resulted in quantal $\mathrm{Ca}^{2+}$ increases with fixed amplitude of approximately $4 \mu \mathrm{M}$. [Note: Average $\mathrm{Ca}^{2+}$ levels depend on the sampled region around the cluster and for an area of $2 \mu \mathrm{m}^{2}$ the amplitude was $\left.1.8 \mu \mathrm{M}\right]$. Long inactivity periods follow these stochastic $\mathrm{Ca}^{2+}$ transient bursts. $\mathrm{Ca}^{2+}$ events in different simulations are shown in Figure. 2.5-D. The $\mathrm{Ca}^{2+}$ transients closely resemble in duration and spread area the fluorescence recording of $\mathrm{Ca}^{2+}$ sparklets in the experiments (Sonkusare et al. 2012; Sonkusare et al. 2014).

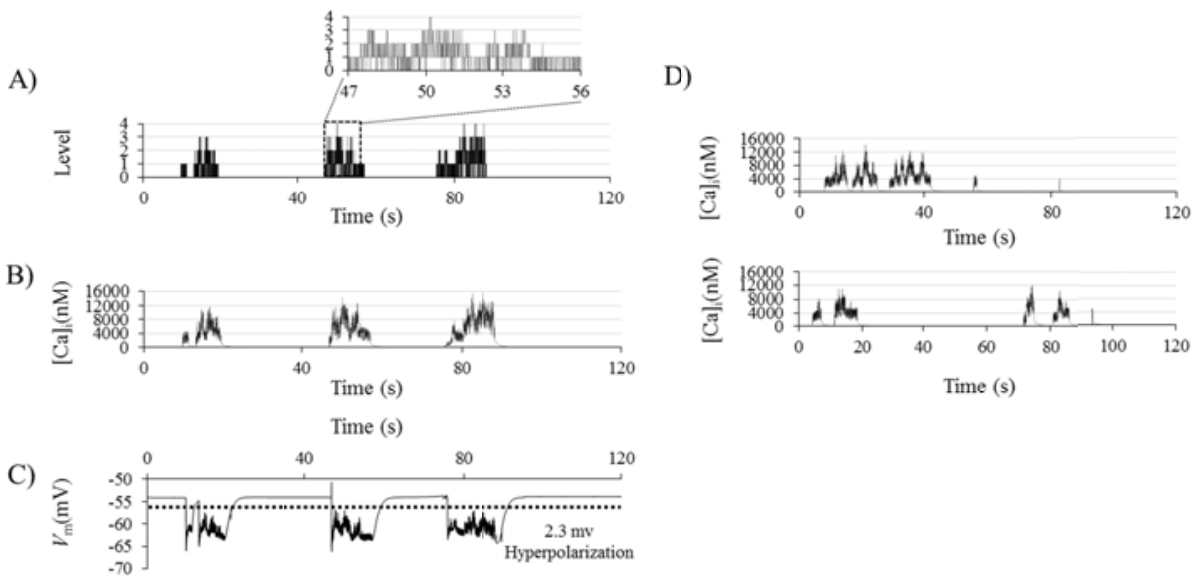

Figure 2.5 Representative example of observed temporal $\mathrm{EC} \mathrm{Ca}^{2+}$ and $\mathrm{EC} V_{m}$ profiles from the stochastic opening of the TRPV4 cluster in the continuum model, (A) Superposition temporal profile of TRPV4 cluster with cooperative gating kinetics implemented using the three-state model. Insert on right shows zoomed in view for better visualization. (B) $\mathrm{EC} \mathrm{Ca}^{2+}$ concentration around the TRPV4 cluster $\left(0.25 \mu \mathrm{m}^{2}\right.$ area) arising from the TRPV4 openings in (A). (C) EC $V_{m}$ transients follow the $\mathrm{EC} \mathrm{Ca}^{2+}$ events. (D) Local EC $\mathrm{Ca}^{2+}$ concentration around the TRPV4 cluster $\left(0.25 \mu \mathrm{m}^{2}\right.$ area) observed in different stochastic simulations. 
The simulated $\mathrm{Ca}^{2+}$ sparklets activated the localized $\mathrm{IK}_{\mathrm{Ca}}$ channels $(25 \%$ of total in the vicinity of the TRPV4s) to produce EC (and SMC) hyperpolarization. EC $V_{\mathrm{m}}$ profile shows hyperpolarization's corresponding to each $\mathrm{Ca}^{2+}$ sparklet (Figure. 2.5-C). An average EC hyperpolarization of around $\sim 2.3 \mathrm{mV}$ was estimated in the 2 minute long simulation.

Simulations were carried out using the alternative model implementations for the TRPV4 cluster openings. Spatial $\mathrm{Ca}^{2+}$ profiles obtained, at the time of maximum $\mathrm{Ca}^{2+}$ spread, using: a) the two-state model with independent channels (Top: No bursting activity), b) the three-state model with independent channels (Middle: bursting activity) and c) the three-state model with interacting channels (Bottom: bursting activity with cooperativity) are depicted color-coded in Figure. 2.6-A. The contour lines represent the region of the $\mathrm{EC}$ where the $\mathrm{Ca}^{2+}$ concentration is above the $\mathrm{IK}_{\mathrm{Ca}} \mathrm{EC}_{50}$. Model simulations with no bursting activity or cooperativity (two-state independent channel model) resulted in approximately $2 \mu \mathrm{m}$ maximum radial distance for half activation of the $\mathrm{IK}_{\mathrm{Ca}}$ channels (Figure. 2.6-B). Bursting activity (three-state independent channel model) and bursting activity with coperativity (three-state cooperative model) increased this radial distance by about 3.2 and 4.5 fold respectively (Figure.2.6-B).

In the model we simulate a single EC coupled to a single SMC. We also assumed that under control conditions, $25 \%$ of the total $\mathrm{IK}_{\mathrm{Ca}}$ channels are localized in close proximity to the TRPV4 cluster (i.e. within $1 \mu \mathrm{m}$ ). This corresponds to $36 \mathrm{IK}_{\mathrm{Ca}}$ channels, based on whole-cell $\mathrm{IK}_{\mathrm{Ca}}$ conductance $\left(G_{I K_{C a}}\right)$ of $1.7 \mathrm{nS}$, single $\mathrm{IK}_{\mathrm{Ca}}$ channel conductance of $17 \mathrm{pS}$, and open probability of 0.7 (Bychkov et al. 2002; Silva, Kapela, and Tsoukias 2007). 

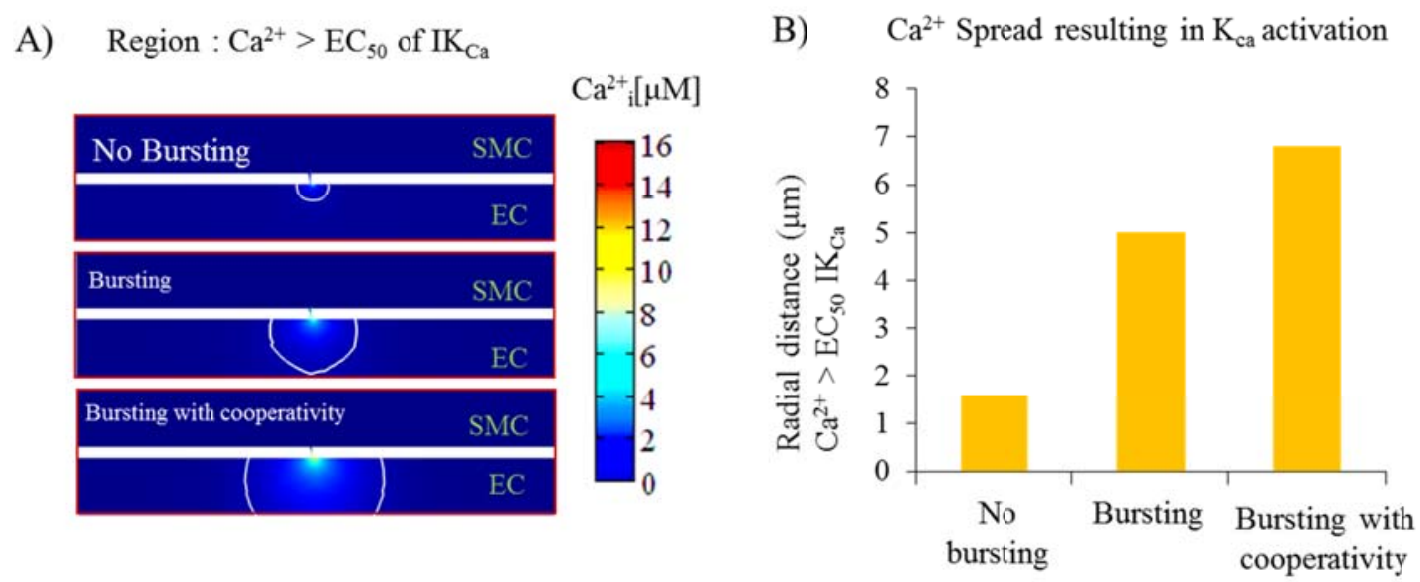

Figure 2.6 Increase in distance of $\mathrm{IK}_{\mathrm{Ca}}$ channel activation arising from burst and cooperative gating kinetics in the TRPV4 cluster, (A) $\mathrm{Ca}^{2+}$ concentration profile in the $\mathrm{EC}$ and the SMC at the time of maximum $\mathrm{Ca}^{2+}$ spread. TRPV4 channel cluster implemented with a two-state model (top: no bursting or cooperative gating kinetics), three-state model (middle: bursting activity) and three-state model with channel interactions (bottom: bursting and cooperativity). Contour lines indicate the $\mathrm{Ca}^{2+}$ concentration equivalent to $\mathrm{EC}_{50}$ of $\mathrm{IK}_{\mathrm{Ca}}$ channels. (B) Radial distance for half-maximum $\mathrm{K}_{\mathrm{Ca}}$ channel activation in the simulations in $(\mathrm{A})$.

Figure. 2.7-A shows temporal changes in the EC $V_{\mathrm{m}}$ induced by the TRPV4 $\mathrm{Ca}^{2+}$ sparklet in a representative simulation. [Note: Similar levels of SMC hyperpolarization were obtained for a MEGJ resistance of $0.9 \mathrm{G} \Omega$ (Yamamoto et al. 2001; Kapela, Bezerianos, and Tsoukias 2009)]. Mean EC $V_{\mathrm{m}}$ hyperpolarization of around $6 \mathrm{mV}$ is predicted during sparklet activity. Under the assumed arrangement of the TRPV4 and $\mathrm{IK}_{\mathrm{Ca}}$ channels in the model, second, third and fourth level TRPV4 opening resulted in $\sim 1.5-4.5 \mathrm{mV}$ depolarization, attenuating the $\mathrm{Ca}^{2+}$ induced hyperpolarization.

In vivo, the hyperpolarization induced by TRPV4 sparklet in individual ECs will spread through homocellular gap junctions to neighboring ECs and through MEGJs to SMCs, causing vessel hyperpolarization and dilation. The bursting response in an individual cell from the $\mathrm{IK}_{\mathrm{Ca}}$ channel activation seen in the model (Figure. 2.5-C, 2.7-A) will be smoothed giving rise to a steady change in vessel's membrane potential. The mean hyperpolarization of the endothelium will be determined by the frequency and 
duration of the sparklet events. Assuming TRPV4 sparklets with a mean duration, $\tau_{\text {burst }}$, an average frequency of occurrence per EC site, $f_{\text {burst }}$, and an average number of active sites per EC, $n_{\text {sites }}$, yields an average number of ECs, $\mathrm{N}=1 /\left(\tau_{\text {burst }} \cdot f_{\text {burst }} \cdot n_{\text {sites }}\right)$, having an active event and open $\mathrm{IK}_{\mathrm{Ca}}$ channels at any given time.

A)

\section{EC-SMC model}

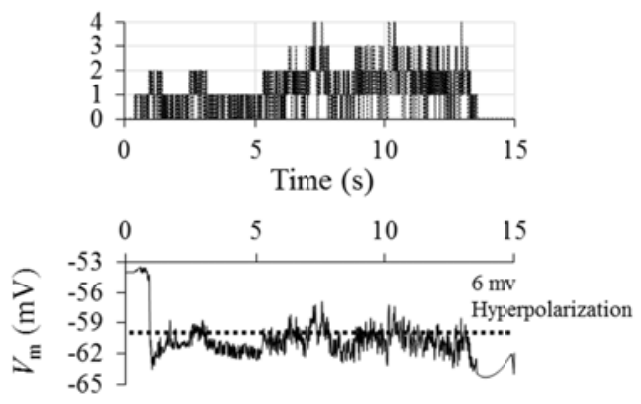

B)

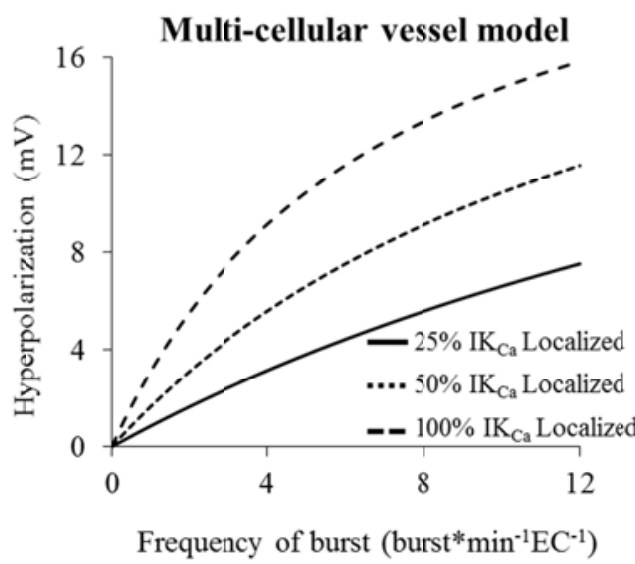

Figure 2.7 Predicted $V_{\mathrm{m}}$ hyperpolarization induced by a localized $\mathrm{Ca}^{2+}$ increase through TRPV4 channels $\left(\mathrm{Ca}^{2+}\right.$ sparklet). (A) Temporal EC $V_{\mathrm{m}}$ profile (bottom) indicates an average EC hyperpolarization of $\sim 6 \mathrm{mV}$ during the bursting activity of the TRPV4 cluster (top) in the single EC- single SMC model. (B) Hyperpolarization of the endothelium as a function of sparklet frequency and $\mathrm{IK}_{\mathrm{Ca}}$ localization, predicted for an intact vessel with EC and SMC layers coupled by MEGJ.

Thus, hyperpolarizing current from each sparklet will spread, on average, in N cells. The resulting hyperpolarization of the endothelium can be approximated from a simplified electrical equivalent of an intact vessel segment (Figure. 2.10) (Equation. 2.17 in the section 2.8). For a representative scenario of $\tau_{\text {burst }}=5 \mathrm{sec}, f_{\text {burst }}=2$ bursts per min per active site and one active site per cell $\mathrm{n}_{\text {sites }}=1$ (i.e. $\mathrm{N}=6$ ), $I K_{\mathrm{Ca}}$ conductance $g_{I K_{C a}}=\gamma_{I K_{C a}}$. $G_{I K_{C a}}=0.25 \times 1.72 \mathrm{nS}$ (representing $25 \%$ of $\mathrm{IK}_{\mathrm{Ca}}$ channels or 36 channels activated by a single TRPV4 sparklet), and net EC-SMC membrane resistance $R_{m}=$ 
$\frac{1}{1 / R_{m}^{E C}+1 /\left(R_{m}^{S M C}+R_{m}^{M E}\right)}$ of $\sim 1.2-2.2 \mathrm{G} \Omega$ (for $R_{m}{ }^{E C}=2-10 G \Omega, R_{m}{ }^{S M C}=2 G \Omega$ and $R_{m}{ }^{M E}=0.9 G \Omega$; (Nilius and Droogmans 2001; Yamamoto et al. 2001; Nagaraja, Kapela, and Tsoukias 2012; Kapela et al. 2011; Yuan et al. 1993)), the estimated vessel hyperpolarization is $\Delta V_{\mathrm{m}} \approx 2-3.4 \mathrm{mV}$ (Equation. 2.17). Figure. 2.8-B shows how this hyperpolarization can increase as a function of the sparklet frequency per $\mathrm{EC}\left(f_{\text {burst }} \cdot n_{\text {sites }}\right)$ and the fraction of $\mathrm{IK}_{\mathrm{Ca}}\left(\gamma_{I K_{C a}}\right)$ localized around the TRPV4 cluster. In the presence of 36 (25\% of total), $72(50 \%)$ and $144(100 \%)$ localized $\mathrm{IK}_{\mathrm{Ca}}$ channels around the TRPV4 cluster, the TRPV4 mediated $\mathrm{Ca}^{2+}$ sparklet (with assumed mean duration of $5 \mathrm{sec}$ ) results in a vessel hyperpolarization of $0.3-15 \mathrm{mV}$ for sparklet frequency between $0.1-12$ bursts/min per EC (Figure. 2.7-B). Similar results were obtained with a multicellular computational model of an intact vessel segment (Kapela, Nagaraja, and Tsoukias 2010). The vessel model predicted a similar $\Delta V_{\mathrm{m}}$ value of $2.3 \mathrm{mV}$ in the endothelium [simulating opening of $25 \%$ of $\mathrm{IK}_{\mathrm{Ca}}$ channels in one $\mathrm{EC}$ out of $6 \mathrm{ECs}$ (i.e. $\mathrm{N}=6$ )]. According to Equation. 2.17, hyperpolarization also depends on the membrane resistance, and in microvessels with higher input resistance, achieved levels of hyperpolarization may be greater relative to larger vessels.

\subsection{Discussion}

The primary aim of the study was to examine the regulatory mechanism of EC TRPV4 channels in inducing vessel relaxation. Using the 2D EC-SMC continuum model coupled with MP, we examined the localized $\mathrm{Ca}^{2+}$ events arising from activation of a single and a cluster of TRPV4 channels in the EC and the resulting vasodilatory response. 
Simulations showed the effect of TRPV4 and $\mathrm{IK}_{\mathrm{Ca}}$ channel distribution, and burst and cooperative gating kinetics of TRPV4 channels in defining the localized $\mathrm{Ca}^{2+}$ event (sparklet), and the resulting SMC hyperpolarization.

Endothelial control of vascular tone is attributed to $\mathrm{EC} \mathrm{Ca}^{2+}$ mobilization that may manifest as events with different spatiotemporal characteristics. Agonist or mechanical stimulation, for example, may increase global $\mathrm{Ca}^{2+}$ levels, the frequency of $\mathrm{Ca}^{2+}$ waves/oscillations, the presence and frequency of $\mathrm{Ca}^{2+}$ pulsars (localized mediated by $\mathrm{IP}_{3} \mathrm{Rs}$ in the vicinity of MP (Ledoux et al. 2008; Nilius and Droogmans 2001)), or of $\mathrm{Ca}^{2+}$ sparklets (events mediated by EC TRPV4 channels (Bagher et al. 2012; Sonkusare et al. 2012; Sonkusare et al. 2014)). Recent evidence suggests a central role of TRPV4 channels in agonist and mechanical induced vasoactive signaling in the microcirculation (Bagher et al. 2012; Sonkusare et al. 2012; Sonkusare et al. 2014).

\subsubsection{TRPV4 $\mathrm{Ca}^{2+}$ sparklet}

The model predicts local micromolar $\mathrm{Ca}^{2+}$ increases from a single TRPV4 channel opening for the duration of the channel's open time (Figure. 2.3). The obtained $\mathrm{Ca}^{2+}$ levels in a small region around the TRPV4 channel are significantly higher than the nanomolar global $\mathrm{Ca}^{2+}$ increases usually observed experimentally (Ledoux et al. 2008; Nilius and Droogmans 2001) or predicted theoretically (Silva, Kapela, and Tsoukias 2007) in response to agonist or mechanical stimuli. These high local $\mathrm{Ca}^{2+}$ concentrations may be required to fully activate cellular components with low $\mathrm{Ca}^{2+}$ affinity including $\mathrm{IK}_{\mathrm{Ca}}$ channels whose affinity may be in the high nanomolar range (reported $\mathrm{EC}_{50}$ values as high as 300-740 $\mathrm{nM}$ (Ahn et al. 2004; Ishii et al. 1997)). 
Multiple TRPV4 channel opening in a cluster resulted in localized quantal $\mathrm{Ca}^{2+}$ increases (Figure. 2.5-B \& 2.5-D). Second, third and fourth level openings in the TRPV4 cluster lead to essentially constant amplitude increases in $\mathrm{Ca}^{2+}$ concentrations that return fast to the previous baseline following channel closure, consistent with the experimental data (Bagher et al. 2012; Sonkusare et al. 2012; Sonkusare et al. 2014). This allows examining channel gating characteristics based on the changes in $\mathrm{Ca}^{2+}$ levels as was done in the experimental studies (Sonkusare et al. 2012; Sonkusare et al. 2014). The model corroborates the methodology utilized in these earlier analyses and shows that $\mathrm{Ca}^{2+}$ diffusion, extrusion and buffering have minimal effect on the interpretation of the data.

In the experiments, TRPV4 channels with an average open time of milliseconds (Sonkusare et al. 2012), generate a $\mathrm{Ca}^{2+}$ burst event (i.e. sparklet) of 1-10 second duration, followed by a long inactivity period before the occurrence of the next event. The significant increase in duration of the $\mathrm{Ca}^{2+}$ event, compared to channel open times, can be a result of bursting activity of the TRPV4 channel. Bursting kinetics of TRPV4s have been observed in single channel patch clamp data (Loukin et al. 2010; Watanabe et al. 2002). Model implementation of bursting in a cluster of four TRPV4 channels (Figure. $2.4 \& 2.5)$ resulted in $\mathrm{Ca}^{2+}$ events lasting for seconds followed by longer inactivity periods (Figure. 2.5-B \& 2.5-D) as observed in the experiments. Burst activity in a cluster of independent TRPV4 channels enhanced the $\mathrm{Ca}^{2+}$ spread and activated $\mathrm{IK}_{\mathrm{Ca}}$ channels at longer distances $\left(\mathrm{Ca}^{2+}\right.$ concentrations $>50 \%$ activation within $\left.5 \mu \mathrm{m}\right)$ (Figure. 2.6-A and 2.6-B). 
Cooperative activation of TRPV4 channels in a cluster has been suggested in experiments as multilevel openings appear much more frequently than suggested by the binomial distribution for random events (Sonkusare et al. 2012). We have accounted for cooperativity in channel kinetics, capturing the increased probability of multi-level openings seen in the experiments. Cooperativity further enhances the $\mathrm{Ca}^{2+}$ spread. The combined effect of bursting and cooperativity is a $\mathrm{Ca}^{2+}$ spread of approximately $7 \mu \mathrm{m}$ (defined as the radial distance with $\mathrm{Ca}^{2+}$ levels greater than $\mathrm{IK}_{\mathrm{Ca}} \mathrm{EC}_{50}$ ). Thus simulations suggest that the gating characteristics of TRPV4 cluster increase the sparklet's area and presumably the resulting hyperpolarization by the recruitment of additional $\mathrm{IK}_{\mathrm{Ca}}$ channels. It will be interesting to examine the effect of clustering of other TRP channel such as of TRPA1- and TRPV3 which have been shown to facilitate endotheliumdependent dilation in cerebral arteries (Earley, Gonzales, and Crnich 2009; Earley, Gonzales, and Garcia 2010). The coupled stochastic-continuum model presented here could be extended towards the analysis of experimental data from stimulation of such channels.

\subsubsection{TRPV4 mediated hyperpolarization}

$\mathrm{EC}$ hyperpolarization through the activation of $\mathrm{IK}_{\mathrm{Ca}}$ and $\mathrm{SK}_{\mathrm{Ca}}$ channels is a major contributor in NO-independent endothelium-derived vasodilation in microvessels (Garland, Hiley, and Dora 2011). TRPV4 $\mathrm{Ca}^{2+}$ sparklets may activate nearby $\mathrm{IK}_{\mathrm{Ca}}$ channels (Sonkusare et al. 2012; Bagher et al. 2012) to induce hyperpolarization and dilation. We tested the potential of TRPV4 sparklets to mediate EDH responses. The presence of high density cellular components including the $\mathrm{K}_{\mathrm{Ca}}$ channels near microdomain sturctures like MPs have been reported in small resistance vessels 
(Heberlein, Straub, and Isakson 2009; Bagher et al. 2012; Ledoux et al. 2008; Sandow et al. 2009; Tran et al. 2012). Micromolar localized $\mathrm{Ca}^{2+}$ increases around the MPs can potentially fully activate the nearby $\mathrm{K}_{\mathrm{Ca}}$ channels (assuming an $\mathrm{EC}_{50}$ as high as $740 \mathrm{nM}$ ). In the model of a single EC coupled to a single SMC, a TRPV4 sparklet activated colocalized $\mathrm{IK}_{\mathrm{Ca}}$ channels in the vicinity of the cluster to generate about $6 \mathrm{mV}$ of hyperpolarization in the EC (Figure. 2.7-A).

Although cooperative gating in the cluster, increased the $\mathrm{Ca}^{2+}$ spread (Figure. 2.6-A), it showed no significant amplification of hyperpolarization (data not shown). This was attributed to the positioning of a high density of $\mathrm{IK}_{\mathrm{Ca}}$ channels within a very close proximity to the cluster $(<1 \mu \mathrm{m})$. Within this area even a single TRPV4 channel produces saturating $\mathrm{Ca}^{2+}$ concentrations so multi-level opening in a cluster does not offer any additional advantage. On the contrary, at physiological ionic concentrations and around resting $V_{\mathrm{m}}$, significant sodium influx currents (pA) are predicted from each TRPV4 opening. This can result in few $\mathrm{mV}$ depolarization which in the absence of additional $\mathrm{K}_{\mathrm{Ca}}$ channel recruitment will have an attenuating effect on EDH signaling. Thus, the high $\mathrm{Na}^{+}$ influx and the micromolar $\mathrm{Ca}^{2+}$ levels in the vicinity of the TRPV4 cluster arising from a single channel opening suggest that bursting and cooperatively may benefit EDH signaling only if $\mathrm{K}_{\mathrm{Ca}}$ channels are positioned at intermediate distances (3-10 $\left.\mu \mathrm{m}\right)$ away from the cluster. Under the assumed arrangement of TRPV4 and $\mathrm{IK}_{\mathrm{Ca}}$ channels in the model, we observed that second, third and fourth TRPV4 channel opening from cooperative gating kinetics, result in a $\sim 1.5-4.5 \mathrm{mV}$ depolarization (Figure. 2.7-A). 
Sustained vessel hyperpolarization results from the spread of transient hyperpolarization generated in a single EC cell to its neighbors. The achieved vessel hyperpolarization will depend on many parameters including membrane resistances of the EC and SMC layers, the MEGJ resistance, the transient hyperpolarizing current, as well as the frequency and duration of the events. Simulations suggest a significant EC hyperpolarization through activation of TRPV4 channels, which increases with increasing the frequency and duration of the event and the amount of the $\mathrm{K}_{\mathrm{Ca}}$ channels in proximity to the TRPV4 cluster (Figure. 2.7-A). Our calculations suggest that experimentally observed TRPV4 $\mathrm{Ca}^{2+}$ sparklets that typically have an average duration of about $5 \mathrm{sec}$ and frequency of occurrence of 2 bursts/min per EC should induce about $2.0 \mathrm{mV}$ hyperpolarization; assuming that 36 channels ( $25 \%$ of total) are located near the cluster (Figure. 2.7-B). This is lower than what has been observed in experiments $(\sim 10 \mathrm{mV})$ (Sonkusare et al. 2012). This discrepancy may be attributed to a higher number of sparklet events/sites than observed experimentally, a very high number of $\mathrm{K}_{\mathrm{Ca}}$ activated by each sparklet (i.e. the majority of $\mathrm{IK}_{\mathrm{Ca}}$ ) or to mechanisms amplifying TRPV4/IK $\mathrm{Ca}$ mediated hyperpolarization. Moreover, the predicted level of hyperpolarization depends on parameters, such membrane resistances (Equation. 2.17), that have not been accurately characterized, that may change with stimulation or varied between vessels. Particularly as the size of vessel decreases input resistance typically increases and this may result in significantly higher hyperpolarization for a given transmembrane current. 


\subsubsection{Local versus global $\mathrm{Ca}^{2+}$ activity}

It is well established that a variety of stimuli, can elicit global $\mathrm{Ca}^{2+}$ increases in ECs. A typical $\mathrm{Ca}^{2+}$ response has an initial transient that is mediated by store $\mathrm{Ca}^{2+}$ release (with peak concentrations in the order of 0.4-1.5 $\mu \mathrm{M}$ (Nilius and Droogmans 2001; Buchan and Martin 1991)) followed by a lower plateau phase that is sustained by $\mathrm{Ca}^{2+}$ influx through $\mathrm{Ca}^{2+}$ permeable channels in the plasma membrane. This global $\mathrm{Ca}^{2+}$ mobilization is thought to mediate vasoactive signaling, including EDH responses. Recent studies, however, showed activation of $\mathrm{IK}_{\mathrm{Ca}}$ channels by localized $\mathrm{Ca}^{2+}$ events such as pulsars and sparklets (Sonkusare et al. 2014; Sonkusare et al. 2012; Ledoux et al. 2008; Bagher et al. 2012) and a moderate global $\mathrm{Ca}^{2+}$ increase ( $\sim 30 \%$ above resting) by cyclopiazonic acid (CPA) did not lead to a significant activation of $\mathrm{K}_{\mathrm{Ca}}$ channels in (Ledoux et al. 2008). Thus, recent evidence points towards a preferential activation of $\mathrm{IK}_{\mathrm{Ca}}$ channels and EDH response to local rather than global $\mathrm{Ca}^{2+}$ increases.

Is there a mechanism that would allow for a portion of $\mathrm{K}_{\mathrm{Ca}}$ channels to be relatively immune to global $\mathrm{Ca}^{2+}$ mobilization so that their activity to be regulated preferentially/exclusively by localized $\mathrm{Ca}^{2+}$ signaling? One possibility is that compartmentalization of TRPV4 and $\mathrm{K}_{\mathrm{Ca}}$ channels may provide a locally confined, regulatory unit relatively inaccessible to global $\mathrm{Ca}^{2+}$ changes. Restriction of $\mathrm{Ca}^{2+}$ signals to subplasmalemma regions has been previously reported suggesting the presence of cytosolic compartments (Saliez et al. 2008; Nilius and Droogmans 2001; Ledoux et al. 2008; Rizzuto and Pozzan 2006; Brasen, Jacobsen, and Holstein-Rathlou 2012). 
Simulation results provide an alternative possibility. The preferential activation of the $\mathrm{K}_{\mathrm{Ca}}$ channels to local rather than global $\mathrm{Ca}^{2+}$ increases may be a result of significantly higher $\mathrm{Ca}^{2+}$ concentrations in localized events (micromolar levels are predicted in the model (Figure. 2.6-B and 2.6-D)) relative to nanomolar global $\mathrm{Ca}^{2+}$ increases during EC stimulation (Silva, Kapela, and Tsoukias 2007; Nilius and Droogmans 2001) . Provided that the $\mathrm{K}_{\mathrm{Ca}} \mathrm{EC}_{50}$ is in the high nanomolar range, global $\mathrm{Ca}^{2+}$ levels may not be sufficient to fully activate $\mathrm{K}_{\mathrm{Ca}}$ and thus a significant portion of the channels is activated only by the high concentration local $\mathrm{Ca}^{2+}$ event. However, reported values for $\mathrm{IK}_{\mathrm{Ca}}$ affinity for $\mathrm{Ca}^{2+}$ range from approximately $100 \mathrm{nM}$ to $740 \mathrm{nM}$ (Nilius and Droogmans 2001; Ahn et al. 2004; Crane et al. 2003; Ishii et al. 1997). The predicted high local $\mathrm{Ca}^{2+}$ concentrations will be beneficial only if the $\mathrm{EC}_{50}$ is in the high end of this range. [A high $\mathrm{EC}_{50}(740 \mathrm{nM})$ (i.e. low affinity) for $\mathrm{IK}_{\mathrm{Ca}}$ channels was assumed in the simulations].

Interestingly, a high $\mathrm{Ca}^{2+}$ affinity for $\mathrm{IK}_{\mathrm{Ca}}$ channels can make these channels significantly (if not fully) activated by global $\mathrm{Ca}^{2+}$ events. In this case, subsequent TRPV4 activation may result in EC depolarization that will attenuate rather than promote EDH. [Recruitment of saturated $\mathrm{K}_{\mathrm{Ca}}$ channels will not compensate for the depolarizing TRPV4 current]. An intermediate scenario where an $\mathrm{IK}_{\mathrm{Ca}}$ channel is activated by both local events and global transients might also be possible. Further experimentation is required to determine the relative contribution of global and local $\mathrm{Ca}^{2+}$ events on $\mathrm{IK}_{\mathrm{Ca}}$ channel activation. 


\subsection{Limitations}

A number of parameter values utilized have not been previously quantified or may vary between different vessels. In this study, we try to remain consistent with our previous EC-SMC models with respect to parameter values and whole cell currents and examine responses over a range of values for parameters with significant uncertainty. The model is limited by the absence of quantitative data for the spatial distribution of important cellular components. Such data may significantly improve the predictive ability of the proposed models. The TRPV4 channel current was modeled using a GHK equation and ignores the effects of temperature, shear and modulatory pathways on the channel. Moreover simple Markov chain models were considered for simplicity and can capture the observed channel behavior, however, the actual gating mechanism could be more complex.

\subsection{Conclusions}

The developed model describes TRPV4 mediated signaling for the regulation of vessel dilation. Model simulations are in good agreement with experiments in isolated vessels, corroborating data for the role of TRPV4 signaling in vascular control. The model predicts a micromolar localized $\mathrm{Ca}^{2+}$ increase through activation of cluster of four TRPV4 channels. This is significantly higher than the nanomolar global $\mathrm{Ca}^{2+}$ levels typically observed during EC stimulation. The model shows an enhanced $\mathrm{Ca}^{2+}$ spread as a result of burst opening and cooperative channel gating in the TRPV4 cluster. $\mathrm{Ca}^{2+}$ mobilization over several micron radial distances can recruit $\mathrm{K}_{\mathrm{Ca}}$ channels and produce millivolts of EDH. Model predicts that amplification of EDH signaling by cooperativity in the TRPV4 cluster is more likely if $\mathrm{K}_{\mathrm{Ca}}$ channels are not immediately adjacent to the 
TRPV4 channels, but at distances that can reach 10 microns away. The magnitude of the resulting hyperpolarization, dependents on a variety of parameters including the sparklet frequency and the effective membrane resistance of the vessel wall. Nevertheless, for a wide range of parameter values predicted TRPV4-induced hyperpolarization's underestimate what has been recorded in some experiments. This may suggest additional mechanisms contributing to the observed EDH response.

\subsection{Supplement}

\subsubsection{Transition rate matrix}

Simulating single-channel behavior can be achieved through matrix formulations which describes the rates of transition in between plausible kinetic states that a channel could reside, as illustrated in (Colquhoun and Hawkes 1982). Consider a continuous time Markov chain with state space $(1,2, \ldots, r)$, the $\mathrm{r} x \mathrm{r}$ transition rate matrix $(\mathrm{Q})$ is defined as $\mathrm{Q}=\left[\mathrm{q}_{\mathrm{ij}}\right]$, where, $\mathrm{q}_{\mathrm{ij}}$ is the transition rate from state $\mathrm{i}$ to state $\mathrm{j}$ and the diagonal elements $\mathrm{q}_{\mathrm{ii}}$ satisfy,

$$
q_{i i}=-\sum_{i \neq j, j=1}^{r} q_{i j}
$$

Equation 2.9

The probability of transition from state $\mathrm{i}$ to state $\mathrm{j}\left(\pi_{i j}\right)$ is given by,

$$
\pi_{i j}=-\frac{q_{i j}}{q_{i i}}
$$

Equation 2.10 
For the simplest two state model with one open and one closed state as described in Mechanism 1 the transition rate matrix is defined as,

$$
\begin{aligned}
& 1: O \quad 2: C
\end{aligned}
$$

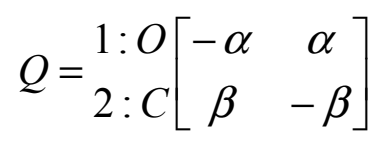

$$
\begin{aligned}
& \text { Equation } 2.11
\end{aligned}
$$

Similarly for the three state model (Equation. 2.6), the transition rate matrix is given by, Equation. 2.12

$$
\begin{aligned}
& 1: O \quad 2: B \quad 3: S \\
& Q=2: B\left[\begin{array}{ccc}
-\left(k_{3}+k_{1}\right) & k_{1} & k_{3} \\
k_{2} & -k_{2} & 0 \\
k_{4} & 0 & -k_{4}
\end{array}\right] \\
& \text { Equation } 2.12
\end{aligned}
$$

\subsubsection{Reduced transition rate matrix}

A system of two channels implemented with two state model will result in a 4 x 4 transition matrix. However a system of four channels implemented with three state model will result in $81 \times 81$ matrix and necessities the formation of reduced transition matrix to implement the behavior of a group of channels. A approach similar to described in Ball et al.(Ball and Rice 1992) was undertaken to obtain a reduced state matrix for a system of four TRPV4 channel described by the three state model. As an example let's consider a system of two channel described using the two state kinetic model of Mechanism 1. The number of possible states are $2^{2}$ resulting in $2^{2} \times 2^{2}$ transition matrix (Equation. 2.13) 


$$
\begin{aligned}
& Q_{2 \text { channels }}= {\left[\begin{array}{ccccc} 
& O_{2} O_{1} & O_{2} C_{1} & C_{2} O_{1} & C_{2} C_{1} \\
O_{2} O_{1} & Q_{o_{2} o_{2}} Q_{o_{1} o_{1}} & Q_{o_{2} o_{2}} Q_{o_{1} c_{1}} & Q_{o_{2} c_{2}} Q_{o_{1} o_{1}} & Q_{o_{2} c_{2}} Q_{o_{1} c_{1}} \\
O_{2} C_{1} & Q_{o_{2} o_{2}} Q_{c_{1} o_{1}} & Q_{o_{2} o_{2}} Q_{c_{1} c_{1}} & Q_{o_{2} c_{2}} Q_{s_{1} o_{1}} & Q_{o_{2} c_{2}} Q_{c_{1} c_{1}} \\
C_{2} O_{1} & Q_{c_{2} o_{2}} Q_{o_{1} o_{1}} & Q_{c_{2} o_{2}} Q_{o_{1} c_{1}} & Q_{c_{2} c_{2}} Q_{o_{1} o_{1}} & Q_{c_{2} c_{2}} Q_{o_{1} c_{1}} \\
C_{2} C_{1} & Q_{c_{2} o_{2}} Q_{c_{1} o_{1}} & Q_{c_{2} o_{2}} Q_{c_{1} c_{1}} & Q_{c_{2} c_{2}} Q_{c_{1} o_{1}} & Q_{c_{2} c_{2}} Q_{c_{1} c_{1}}
\end{array}\right] } \\
& Q_{2 \text { channels }}=\left[\begin{array}{cccc}
-2 \alpha & \alpha & \alpha & 0 \\
\beta & -(\alpha+\beta) & 0 & \alpha \\
\beta & 0 & -(\alpha+\beta) & \alpha \\
0 & \beta & \beta & -2 \beta
\end{array}\right]
\end{aligned}
$$

Equation 2.13

Of the four possible states i.e. both channels open $\left(\mathrm{O}_{1} \mathrm{O}_{2}\right)$, one channel open and other closed $\left(\left(\mathrm{O}_{2} \mathrm{C}_{1}\right),\left(\mathrm{C}_{2} \mathrm{O}_{1}\right)\right)$ and both channels closed $\left(\mathrm{C}_{1} \mathrm{C}_{2}\right)$, the state $\left(\mathrm{O}_{2} \mathrm{C}_{1}\right)$ and $\left(\mathrm{C}_{2} \mathrm{O}_{1}\right)$ are indistinguishable as it is difficult to determine which of the two channels is in the open state. Utilizing these symmetries and considering only the number of channels in each state it is possible to reduce the size of the state space to 3 states (Equation. 2.14) or in general for a $\mathrm{N}$ channel system described using $\mathrm{r}$ state model reduces to $\left(\begin{array}{c}N+r-1 \\ N\end{array}\right)$ (Ball and Rice 1992).

$$
Q_{2 \text { channels }}=\left[\begin{array}{cccc}
O O & O C & C C \\
O O & Q_{O O O O} & 2 Q_{O O O C} & Q_{O O C C} \\
O C & Q_{O C O O} & Q_{O C O C} & Q_{O C C C} \\
C C & Q_{C С O O} & 2 Q_{C C O C} & Q_{C C C C}
\end{array}\right] \Rightarrow Q_{2 \text { channels }}=\left[\begin{array}{ccc}
-2 \alpha & 2 \alpha & o \\
\beta & -\alpha+\beta & \alpha \\
0 & 2 \beta & -2 \beta
\end{array}\right]
$$

Equation 2.14

We utilized similar approach for a system of four channels described by the three state model to reduce it to a $15 \times 15$ matrix given by Equation. 2.15, where the number is the brackets in the first row represent the number of symmetrical states to the corresponding 
state provided in the second row. For example state 7 in second row (OBBB) has four other symmetrical states depending on which of channel one to four is in the open state. The diagonal elements $\left(\mathrm{d}_{\mathrm{i}}\right)$ are equal to negative of sum of elements in row $i$, where $\mathrm{i}=1,2,3 \ldots 15$.

$\begin{array}{cccccccccccccccc} & (1) & (4) & (4) & (6) & (12) & (6) & (4) & (12) & (12) & (4) & (1) & (4) & (6) & (4) & (1) \\ Q_{4 c h a n n e l s}= & 1 & 2 & 3 & 4 & 5 & 6 & 7 & 8 & 9 & 10 & 11 & 12 & 13 & 14 & 15 \\ O O O O B: 1 & d_{1} & 4 k_{1} & 4 k_{3} & 0 & 0 & 0 & 0 & 0 & 0 & 0 & 0 & 0 & 0 & 0 & 0 \\ O B B: 7 & 0 & 0 & 0 & 6 k_{2} & 0 & 0 & d_{7} & 0 & 0 & 0 & k_{1} & 4 k_{3} & 0 & 0 & 0 \\ O O O B: 2 & k_{2} & d_{2} & 0 & 6 k_{1} & 12 k_{3} & 0 & 0 & 0 & 0 & 0 & 0 & 0 & 0 & 0 & 0 \\ O O O S: 3 & k_{4} & 0 & d_{3} & 0 & 12 k_{1} & 6 k_{3} & 0 & 0 & 0 & 0 & 0 & 0 & 0 & 0 & 0 \\ O O B B: 4 & 0 & 4 k_{2} & 0 & d_{4} & 0 & 0 & 4 k_{1} & 12 k_{3} & 0 & 0 & 0 & 0 & 0 & 0 & 0 \\ O O B S: 5 & 0 & 4 k_{4} & 4 k_{2} & 0 & d_{5} & 0 & 0 & 12 k_{1} & 12 k_{3} & 0 & 0 & 0 & 0 & 0 & 0 \\ O B S S: 9 & 0 & 0 & 0 & 0 & 12 k_{4} & 6 k_{2} & 0 & 0 & d_{9} & 0 & 0 & 0 & 6 k_{1} & 4 k_{3} & 0 \\ O S S S: 10 & 0 & 0 & 0 & 0 & 0 & 6 k_{4} & 0 & 0 & 0 & d_{10} & 0 & 0 & 0 & 4 k_{1} & k_{3} \\ B B B B: 11 & 0 & 0 & 0 & 0 & 0 & 0 & 4 k_{2} & 0 & 0 & 0 & d_{11} & 0 & 0 & 0 & 0 \\ B B B S: 12 & 0 & 0 & 0 & 0 & 0 & 0 & 4 k_{4} & 12 k_{2} & 0 & 0 & 0 & d_{12} & 0 & 0 & 0 \\ B B S S: 13 & 0 & 0 & 0 & 0 & 0 & 0 & 0 & 12 k_{4} & 12 k_{2} & 0 & 0 & 0 & d_{13} & 0 & 0 \\ B S S S: 14 & 0 & 0 & 0 & 0 & 0 & 0 & 0 & 0 & 12 k_{4} & 4 k_{2} & 0 & 0 & 0 & d_{14} & 0 \\ S S S S: 15 & 0 & 0 & 0 & 0 & 0 & 0 & 0 & 0 & 0 & 4 k_{4} & 0 & 0 & 0 & 0 & d_{15}\end{array}$

Equation 2.15

\subsubsection{Cooperativity implementation}

Consider system of two interacting channels as illustrated in (Keleshian et al. 1994) each described by a simple two state model (Mechanism 1) with transition rates $\alpha$ and $\beta$. The channels have an opening rate $\beta^{\prime}$ during a period in which the other channel is open, and $\beta$ otherwise. The conditional densities can be written as in Equation. 2.16, where, for example, $f_{2 c \mid 1 c}$ is the density of closed sojourn time of second channel given that the first channel is in closed state. 


$$
\begin{aligned}
& f_{2 c \mid 1 c}=f_{1 c \mid 2 c}=\beta e^{-\beta t} \\
& f_{2 o \mid 1 o}=f_{2 o \mid 1 c}=f_{1 c \mid 2 o}=f_{1 c \mid 2 c}=\alpha e^{-\alpha t} \\
& f_{2 c \mid 1 o}=f_{1 c \mid 2 o}=\beta^{\prime} e^{-\beta^{\prime} t}\left(\beta^{\prime}>\beta\right)
\end{aligned}
$$

Equation 2.16

To implement cooperativity we modified the reduced transition matrix defined in Equation. 2.14 and Equation. 2.15 through increasing the parameter value of $\beta$ (to $\beta^{\prime}$ ) and $\mathrm{k}_{4}\left(\mathrm{to}_{4}{ }^{\prime}\right)$ respectively during presence of at least one channel in open state, i.e. we increase the probability of opening of a channel in a shut or a blocked state only in the presence of one or more open channels in a cluster. For example, in Equation. 2.15 consider the transition from state OOOS to OOOO. We increase the parameter $\mathrm{k}_{4}$ as transition of the only shut channel occurs to open state in the presence of three open channels, however for the transition from SSSS to OSSS state the parameter $\mathrm{k}_{4}$ in the transition matrix would not be modified as one of the shut channel transits to open state in the presence of three other channels which are all in shut state. The values of $\beta^{\prime}$ and $\mathrm{k}_{4}{ }^{\prime}$ were chosen to obtain an $\mathrm{NP}_{\mathrm{o}}$ observed in the experiments (Sonkusare et al. 2012) as shown in Figure. 2.8.

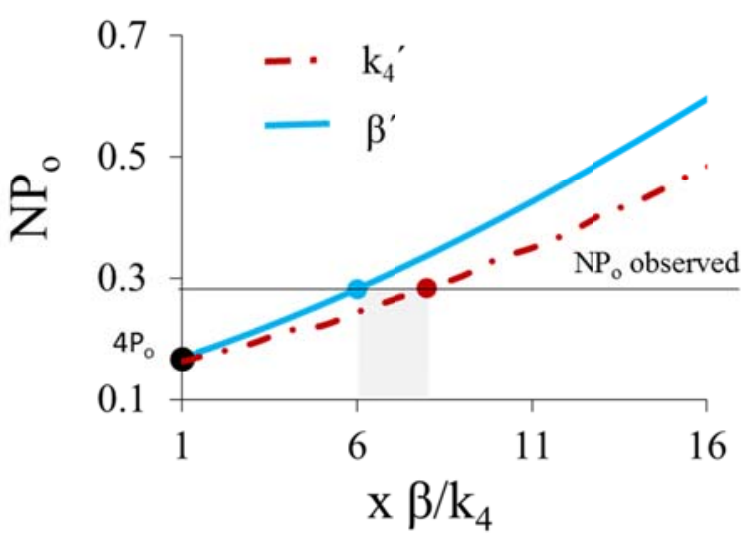

Figure 2.8 Plot of $\mathrm{NP}_{\mathrm{o}}$ obtained with 1-20 fold increase in beta (yellow line) and $\mathrm{k}_{4}$ (red dotted line) 


\subsubsection{Stochastic TRPV4 opening (Two-state model)}

Stochastic opening of a TRPV4 channel was simulated using a transition rate matrix (Equation. 2.11) with $\alpha=2.7 \times 10^{-2} \mathrm{~ms}^{-1}$ and $\beta=1.3 \times 10^{-3} \mathrm{~ms}^{-1}$. A representative single channel record of 120 second duration is shown in Figure. 2.9-A, displaying random opening and closing of the TRPV4 channel with an overall $\mathrm{P}_{\mathrm{o}}$ of 0.045 . The histogram of the dwell time in the open state $(\mathrm{O})$ was constructed (data not shown) and matched the experimentally observed channel open time distribution in (Sonkusare et al. 2012).

Superposition records of a cluster of four TRPV4 channels, with each individual channel represented by the two-state model (Mechanism 1), were simulated using the reduced two-state four-channel transition rate matrix (Equation. 2.14) with $\alpha\left(2.7 \times 10^{-2} \mathrm{~ms}^{-1}\right)$ and $\beta\left(1.3 \times 10^{-3} \mathrm{~ms}^{-1}\right)$ as before. A two min long representative simulation of a cluster of four independent TRPV4 channels is shown in Figure. 2.9-B. A few second and third level opening are observed with overall $\mathrm{NP}_{\mathrm{o}}$ of 0.17 . Cooperative opening in a cluster of four TRPV4 channels was simulated through increasing the transition rate $\beta$ to $\beta^{\prime}$ in the presence of at least one open channel in the cluster (data not shown). A range of $\beta^{\prime}$ was examined (Figure. 2.8) and $\beta^{\prime}$ equal to $6 \beta$ was used in the model to match the experimentally observed $\mathrm{NP}_{\mathrm{o}}$ value $(\sim 0.27)$.

In a cluster of four independent TRPV4 channel, the probabilities for one, two, three or four channels being open at a time (Figure. 2.9-C, Grey checkered bars) followed closely the expected binomial distribution with a single channel $\mathrm{P}_{\mathrm{o}}$ of 0.045 (Figure. 2.9C, Grey solid bars). 
Simulations with a cluster of TRPV4s with cooperative gating revealed significantly higher probabilities for second, third and fourth level opening (Figure. 2.9-C, Black checkered bars) that closely follow those observed in experiments (Figure. 2.9-C, Black solid bars).

A)

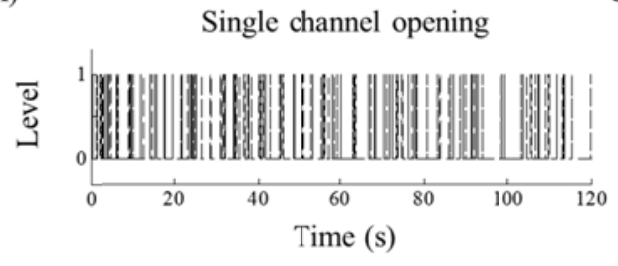

B)

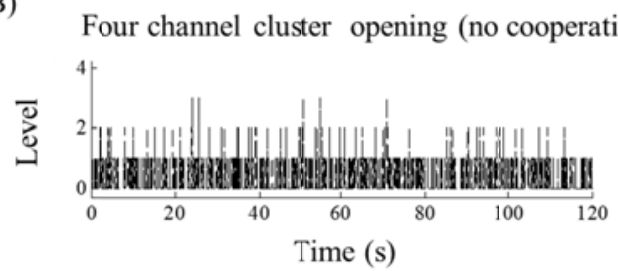

C)

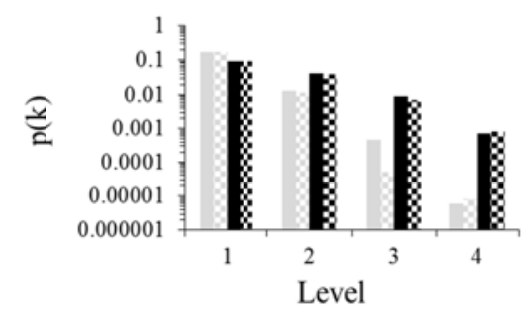

$\begin{array}{ll}\text { Binomial } & \mathbf{m} \text { Experiment } \\ \text { No cooperativity model } & \mathrm{x} \text { Cooperativity model }\end{array}$

Figure 2.9 Stochastic opening of TRPV4 channels described by a two-state Markov chain. (A) Illustrative example of a temporal profile of a single TRPV4 channel transitioning between the close and the open states. (B) A superposition temporal profile of a cluster of four independent TRPV4 channels. The level number describes the number of open channels at a given time point. (C) Open probabilities of first, second, third and fourth level channel openings in a TRPV4 cluster of four channels. The two-state Markov model considering independent channels (grey checkered bars) matches the binomial distribution of independent events (grey solid bar; Equation. 2.7, $\mathrm{P}_{\mathrm{o}}=0.045$ ). Experimental data from (Sonkusare et al. 2012) (black solid bar) show increased probability of second, third, and fourth channel openings relative to the binomial distribution. The two-state model with cooperative channel gating (black checkered bar; $\beta^{\prime}=$ $6 \beta$ ) matches the experimentally observed open probabilities.

\subsubsection{Electrical equaivalent of multi-cellular vessel segment}

Figure 2.10 shows a simplified electrical equivalent circuit used to estimate the hyperpolarization of the endothelium in intact vessels induced by the activity of TRPV4.

From Figure. 2.10

$$
\Delta V_{m}=\frac{E_{K}-V_{r e s t}}{\frac{R_{m}}{N}+\frac{R_{I K_{C a}}}{\gamma_{I K_{C a}}}} \cdot \frac{R_{m}}{N}=\frac{E_{K}-V_{r e s t}}{1+\frac{N \cdot R_{I K_{C a}}}{\gamma_{I K_{C a}} \cdot R_{m}}}
$$


where $\Delta V_{m}$ is the change in endothelial $V_{m}, E_{K}=-79 \mathrm{mV}$ is the Nernst potential for $\mathrm{K}^{+}$, $V_{\text {rest }}=-54 \mathrm{mV}$ is the resting EC $V_{\mathrm{m}}$ coupled to a $\mathrm{SMC}, \mathrm{R}_{\mathrm{m}} \approx 1.2 \mathrm{G} \Omega$ is the net membrane resistance of EC connected through myoendothelial gap junction to SMC, $R_{I K_{C a}}$ is the total resistance of $I K_{\mathrm{Ca}}$ channels, $\gamma_{I K_{C a}}(0-1)$ is the fraction of $I K_{\mathrm{Ca}}$ activated by single TRPV4 sparklet, $N=\frac{1}{\tau_{\text {burst }} \cdot \mathrm{f}_{\text {burst }} \cdot \mathrm{n}_{\text {sites }}}$, is the number of ECs having an active event and open $\mathrm{IK}_{\mathrm{Ca}}$ channels at any given time point., $\tau_{\text {burst }}$ (assumed mean of $5 \mathrm{sec}$ ) is the average duration of TRPV4 sparklet, $f_{\text {burst }}$ is the frequency of TRPV4 $\mathrm{Ca}^{2+}$ burst per site and $\mathrm{n}_{\text {sites }}$ is the number of active sites per EC.

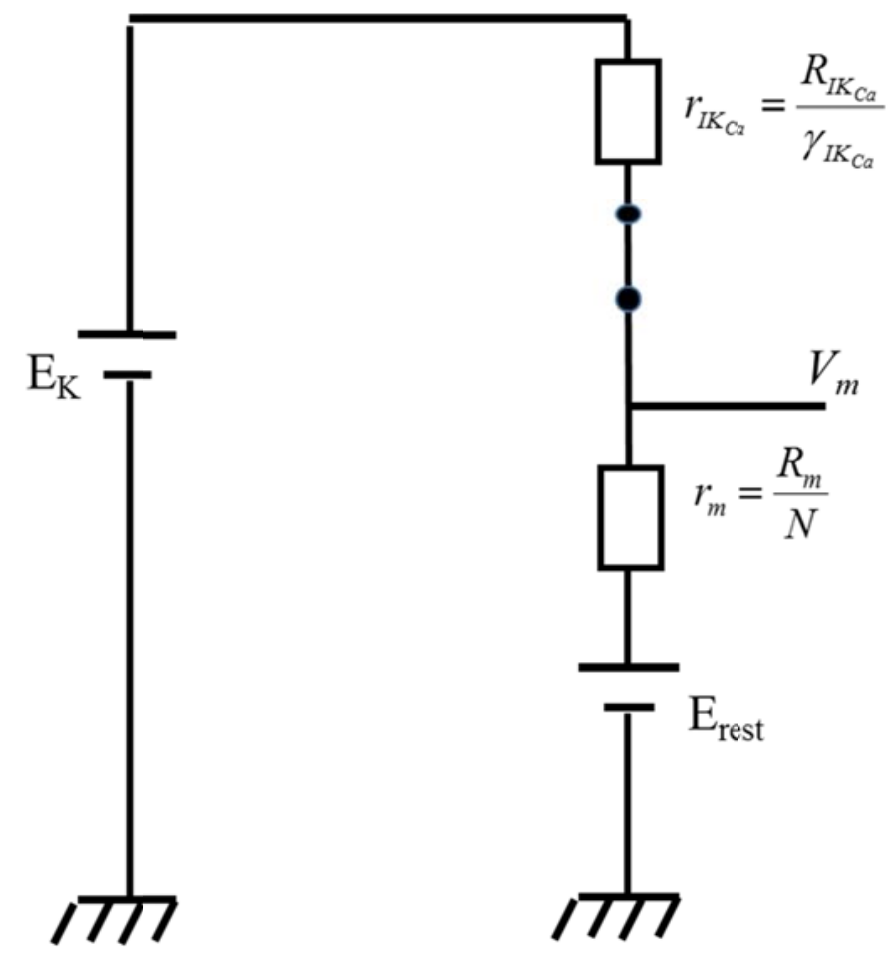

Figure 2.10 Electrical equivalent of a vessel segment with activated $\mathrm{IK}_{\mathrm{Ca}} \cdot r_{m}$ is the net membrane resistance of ECs coupled to SMCs and $\mathrm{R}_{\mathrm{m}}$ is the effective resistance of a EC coupled to a SMC, $R_{I K_{C a}}$ is the total resistance of $\mathrm{IK}_{\mathrm{Ca}}$ channels in a single $\mathrm{EC}$ and $r_{I K_{C a}}$ is the net resistance of $\mathrm{IK}_{\mathrm{Ca}}$ channels in the vessel activated by TRPV4 sparklets. 


\subsection{References}

Ahn, S. C., G. H. Seol, J. A. Kim, and S. H. Suh. 2004. Characteristics and a functional implication of $\mathrm{Ca}(2+)$-activated $\mathrm{K}(+)$ current in mouse aortic endothelial cells. Pflugers Arch 447 (4):426-35.

Bagher, P., T. Beleznai, Y. Kansui, R. Mitchell, C. J. Garland, and K. A. Dora. 2012. Low intravascular pressure activates endothelial cell TRPV4 channels, local $\mathrm{Ca} 2+$ events, and IKCa channels, reducing arteriolar tone. Proc Natl Acad Sci U S A 109 (44):18174-9.

Ball, F. G., and J. A. Rice. 1992. Stochastic models for ion channels: introduction and bibliography. Math Biosci 112 (2):189-206.

Brasen, J. C., J. C. Jacobsen, and N. H. Holstein-Rathlou. 2012. The nanostructure of myoendothelial junctions contributes to signal rectification between endothelial and vascular smooth muscle cells. PloS one 7 (4):e33632.

Buchan, K. W., and W. Martin. 1991. Modulation of agonist-induced calcium mobilisation in bovine aortic endothelial cells by phorbol myristate acetate and cyclic AMP but not cyclic GMP. Br J Pharmacol 104 (2):361-6.

Bychkov, R., M. P. Burnham, G. R. Richards, G. Edwards, A. H. Weston, M. Feletou, and P. M. Vanhoutte. 2002. Characterization of a charybdotoxin-sensitive intermediate conductance $\mathrm{Ca} 2+$-activated $\mathrm{K}+$ channel in porcine coronary endothelium: relevance to EDHF. Br J Pharmacol 137 (8):1346-54.

Colquhoun, D., and A. G. Hawkes. 1981. On the stochastic properties of single ion channels. Proc R Soc Lond B Biol Sci 211 (1183):205-35.

Colquhoun, D., and A. G. Hawkes. 1982. On the stochastic properties of bursts of single ion channel openings and of clusters of bursts. Philos Trans R Soc Lond B Biol Sci 300 (1098):1-59.

Crane, G. J., N. Gallagher, K. A. Dora, and C. J. Garland. 2003. Small- and intermediateconductance calcium-activated $\mathrm{K}+$ channels provide different facets of endothelium-dependent hyperpolarization in rat mesenteric artery. $J$ Physiol 553 (Pt 1):183-9.

Dora, K. A., N. T. Gallagher, A. McNeish, and C. J. Garland. 2008. Modulation of endothelial cell $\mathrm{KCa} 3.1$ channels during endothelium-derived hyperpolarizing factor signaling in mesenteric resistance arteries. Circ Res 102 (10):1247-55.

Draber, S., R. Schultze, and U. P. Hansen. 1993. Cooperative behavior of K+ channels in the tonoplast of Chara corallina. Biophys $J 65$ (4):1553-9. 
Earley, S., A. L. Gonzales, and R. Crnich. 2009. Endothelium-dependent cerebral artery dilation mediated by TRPA1 and Ca2+-Activated K+ channels. Circ Res 104 (8):987-94.

Earley, S., A. L. Gonzales, and Z. I. Garcia. 2010. A dietary agonist of transient receptor potential cation channel V3 elicits endothelium-dependent vasodilation. $\mathrm{Mol}$ Pharmacol 77 (4):612-20.

Earley, S., T. Pauyo, R. Drapp, M. J. Tavares, W. Liedtke, and J. E. Brayden. 2009. TRPV4-dependent dilation of peripheral resistance arteries influences arterial pressure. Am J Physiol Heart Circ Physiol 297 (3):H1096-102.

Edwards, G., M. Feletou, and A. H. Weston. 2010. Endothelium-derived hyperpolarising factors and associated pathways: a synopsis. Pflugers Arch 459 (6):863-79.

Garland, C. J., C. R. Hiley, and K. A. Dora. 2011. EDHF: spreading the influence of the endothelium. Br J Pharmacol 164 (3):839-52.

Heberlein, K. R., A. C. Straub, and B. E. Isakson. 2009. The myoendothelial junction: breaking through the matrix? Microcirculation 16 (4):307-22.

Heller, S., and R. G. O'Neil. 2007. Molecular Mechanisms of TRPV4 Gating.

Isakson, B. E. 2008. Localized expression of an $\operatorname{Ins}(1,4,5) \mathrm{P} 3$ receptor at the myoendothelial junction selectively regulates heterocellular $\mathrm{Ca} 2+$ communication. J Cell Sci 121 (Pt 21):3664-73.

Ishii, T. M., C. Silvia, B. Hirschberg, C. T. Bond, J. P. Adelman, and J. Maylie. 1997. A human intermediate conductance calcium-activated potassium channel. Proc Natl Acad Sci U S A 94 (21):11651-6.

Kapela, A., A. Bezerianos, and N. M. Tsoukias. 2008. A mathematical model of Ca2+ dynamics in rat mesenteric smooth muscle cell: agonist and $\mathrm{NO}$ stimulation. $J$ Theor Biol 253 (2):238-60.

Kapela, A., A. Bezerianos, and N. M. Tsoukias. 2009. A mathematical model of vasoreactivity in rat mesenteric arterioles: I. Myoendothelial communication. Microcirculation 16 (8):694-713.

Kapela, A., S. Nagaraja, J. Parikh, and N. M. Tsoukias. 2011. Modeling Ca2+ signaling in the microcirculation: intercellular communication and vasoreactivity. Crit Rev Biomed Eng 39 (5):435-60.

Kapela, A., S. Nagaraja, and N. M. Tsoukias. 2010. A mathematical model of vasoreactivity in rat mesenteric arterioles. II. Conducted vasoreactivity. $\mathrm{Am} J$ Physiol Heart Circ Physiol 298 (1):H52-65. 
Kapela, A., and N. M. Tsoukias. 2011. Multiscale FEM modeling of vascular tone: from membrane currents to vessel mechanics. IEEE Trans Biomed Eng 58 (12):3456-9.

Keleshian, A. M., G. F. Yeo, R. O. Edeson, and B. W. Madsen. 1994. Superposition properties of interacting ion channels. Biophys $J 67$ (2):634-40.

Kerr, P. M., R. Tam, K. Ondrusova, R. Mittal, D. Narang, C. H. Tran, D. G. Welsh, and F. Plane. 2012. Endothelial feedback and the myoendothelial projection. Microcirculation 19 (5):416-22.

Ledoux, J., M. S. Taylor, A. D. Bonev, R. M. Hannah, V. Solodushko, B. Shui, Y. Tallini, M. I. Kotlikoff, and M. T. Nelson. 2008. Functional architecture of inositol 1,4,5-trisphosphate signaling in restricted spaces of myoendothelial projections. Proc Natl Acad Sci U S A 105 (28):9627-32.

Loukin, S., X. Zhou, Z. Su, Y. Saimi, and C. Kung. 2010. Wild-type and brachyolmiacausing mutant TRPV4 channels respond directly to stretch force. J Biol Chem 285 (35):27176-81.

Ma, X., B. Nilius, J. W. Wong, Y. Huang, and X. Yao. 2011. Electrophysiological properties of heteromeric TRPV4-C1 channels. Biochim Biophys Acta 1808 (12):2789-97.

Mendoza, S. A., J. Fang, D. D. Gutterman, D. A. Wilcox, A. H. Bubolz, R. Li, M. Suzuki, and D. X. Zhang. 2010. TRPV4-mediated endothelial Ca2+ influx and vasodilation in response to shear stress. Am J Physiol Heart Circ Physiol 298 (2):H466-76.

Nagaraja, S., A. Kapela, C. H. Tran, D. G. Welsh, and N. M. Tsoukias. 2013. Role of microprojections in myoendothelial feedback--a theoretical study. J Physiol 591 (Pt 11):2795-812.

Nagaraja, S., A. Kapela, and N. M. Tsoukias. 2012. Intercellular communication in the vascular wall: a modeling perspective. Microcirculation 19 (5):391-402.

Nilius, B., and G. Droogmans. 2001. Ion channels and their functional role in vascular endothelium. Physiol Rev 81 (4):1415-59.

Nilius, B., J. Vriens, J. Prenen, G. Droogmans, and T. Voets. 2004. TRPV4 calcium entry channel: a paradigm for gating diversity. Am J Physiol Cell Physiol 286 (2):C195205 .

Rizzuto, R., and T. Pozzan. 2006. Microdomains of intracellular Ca2+: molecular determinants and functional consequences. Physiol Rev 86 (1):369-408.

Saliez, J., C. Bouzin, G. Rath, P. Ghisdal, F. Desjardins, R. Rezzani, L. F. Rodella, J. Vriens, B. Nilius, O. Feron, J. L. Balligand, and C. Dessy. 2008. Role of caveolar 
compartmentation in endothelium-derived hyperpolarizing factor-mediated relaxation: $\mathrm{Ca} 2+$ signals and gap junction function are regulated by caveolin in endothelial cells. Circulation 117 (8):1065-74.

Sandow, S. L., R. E. Haddock, C. E. Hill, P. S. Chadha, P. M. Kerr, D. G. Welsh, and F. Plane. 2009. What's where and why at a vascular myoendothelial microdomain signalling complex. Clin Exp Pharmacol Physiol 36 (1):67-76.

Sandow, S. L., C. B. Neylon, M. X. Chen, and C. J. Garland. 2006. Spatial separation of endothelial small- and intermediate-conductance calcium-activated potassium channels $(\mathrm{K}(\mathrm{Ca}))$ and connexins: possible relationship to vasodilator function? $J$ Anat 209 (5):689-98.

Silva, H. S., A. Kapela, and N. M. Tsoukias. 2007. A mathematical model of plasma membrane electrophysiology and calcium dynamics in vascular endothelial cells. Am J Physiol Cell Physiol 293 (1):C277-93.

Sonkusare, S. K., A. D. Bonev, J. Ledoux, W. Liedtke, M. I. Kotlikoff, T. J. Heppner, D. C. Hill-Eubanks, and M. T. Nelson. 2012. Elementary Ca2+ signals through endothelial TRPV4 channels regulate vascular function. Science 336 (6081):597601.

Sonkusare, S. K., T. Dalsgaard, A. D. Bonev, D. C. Hill-Eubanks, M. I. Kotlikoff, J. D. Scott, L. F. Santana, and M. T. Nelson. 2014. AKAP150-dependent cooperative TRPV4 channel gating is central to endothelium-dependent vasodilation and is disrupted in hypertension. Sci Signal 7 (333):ra66.

Strotmann, R., G. Schultz, and T. D. Plant. 2003. Ca2+-dependent potentiation of the nonselective cation channel TRPV4 is mediated by a C-terminal calmodulin binding site. J Biol Chem 278 (29):26541-9.

Tran, C. H., M. S. Taylor, F. Plane, S. Nagaraja, N. M. Tsoukias, V. Solodushko, E. J. Vigmond, T. Furstenhaupt, M. Brigdan, and D. G. Welsh. 2012. Endothelial Ca2+ wavelets and the induction of myoendothelial feedback. Am J Physiol Cell Physiol 302 (8):C1226-42.

Watanabe, H., J. Vriens, S. H. Suh, C. D. Benham, G. Droogmans, and B. Nilius. 2002. Heat-evoked activation of TRPV4 channels in a HEK293 cell expression system and in native mouse aorta endothelial cells. J Biol Chem 277 (49):47044-51.

Yamamoto, Y., M. F. Klemm, F. R. Edwards, and H. Suzuki. 2001. Intercellular electrical communication among smooth muscle and endothelial cells in guineapig mesenteric arterioles. $J$ Physiol 535 (Pt 1):181-95. 
Yuan, X. J., W. F. Goldman, M. L. Tod, L. J. Rubin, and M. P. Blaustein. 1993. Ionic currents in rat pulmonary and mesenteric arterial myocytes in primary culture and subculture. Am J Physiol 264 (2 Pt 1):L107-15. 
CHAPTER 3-NITRIC OXIDE (NO) MEDIATED VASODILATORY FEEDBACK RESPONSE AND ITS MODULATION BY ENDOTHELIAL HEMOGLOBIN $\alpha$ AND ERYTHROCYTES

This chapter is to be submitted to Journal of Physiology (with modifications) as Parikh, J., A. Kapela and N. M.Tsoukias. "Localized endothelial nitric oxide-mediated smooth muscle cell hyperpolarization; regulation by endothelial Hemoglobin $\alpha$ and erythrocyte's" 


\subsection{Abstract}

Nitric oxide (NO) derived from endothelial nitric oxide synthase (eNOS) is known to modulate the vessel tone in response to both vasoconstrictors and vasodilators. We utilize mathematical modeling to investigate NO mediated vasodilatory feedback response and address experimental limitations in quantifying vascular NO concentration. We analyze recently reported hypothesis governing NO signaling through consumption by hemoglobin $\alpha(\mathrm{Hb} \alpha)$ colocalized with eNOS in the endothelial microprojections (MP) by Straub et al. (Nature 491: 473-477, 2012). We developed detailed continuum cellular models of $\mathrm{Ca}^{2+}$ dynamics, membrane electrophysiology and NO signaling for coupled smooth muscle (SMC) and endothelial cell (EC). The model takes into consideration the recently reported enriched expression of the $\mathrm{IP}_{3}$ receptors, eNOS, $\mathrm{Ca}^{2+}$ activated potassium $\left(\mathrm{K}_{\mathrm{Ca}}\right)$ channels and $\mathrm{Hb} \alpha$ in the MP. Localized $\mathrm{EC} \mathrm{Ca}^{2+}$ mobilization is induced during SMC stimulation in the MP. NO mediated feedback response through this localized $\mathrm{Ca}^{2+}$ signaling is observed in the model in the presence of localized eNOS in the vicinity of the MP. EC Hba reduced the feedback through the consumption of NO as observed in the experiments. Modulation of NO biovailability by $\mathrm{Hb} \alpha$ is enhanced by their colocalization in the MP. Moreover model simulations suggest that RBC perfusion will decrease the ability of $\mathrm{Hb} \alpha$ to modulate $\mathrm{NO}$ levels, and $\mu \mathrm{M}$ levels of $\mathrm{EC} \mathrm{Hb} \alpha$ are necessary for a significant modulation of SMC NO availability. The model explores the NO signaling mechanism in response to the local $\mathrm{Ca}^{2+}$ mobilization and also aids in quantification of $\mathrm{Hb} \alpha$ concentrations essential for modulating NO mediated feedback response. 


\subsection{Introduction}

In microvasculature, complex bidirectional communication between the endothelial (EC) and smooth muscle cell (SMC) regulates SMC constriction and vascular tone. EC extensions over the internal elastic lamina towards SMC, termed myoendothelial projections (MPs), have been observed in resistance vessels (Heberlein, Straub, and Isakson 2009; Sandow et al. 2009) and can play a role in the modulation of vessel tone (Kerr et al. 2012). Recent studies provide evidence for enriched expression of inositol 1,4,5-triphosphate receptors $\left(\mathrm{IP}_{3} \mathrm{Rs}\right)$, connexins, caveolae, eNOS and Hba in/near MPs (Straub et al. 2011; Straub et al. 2012; Isakson 2008; Ledoux et al. 2008; Heberlein, Straub, and Isakson 2009) to form a powerful signaling unit. Myoendothelial gap junctions (MEGJs) are often found at the tip of the MP, and allow for electrochemical communication between ECs and SMCs (Heberlein, Straub, and Isakson 2009; Sandow et al. 2009). Agonist stimulation of the SMC induces inositol triphosphate ( $\left.\operatorname{IP}_{3}\right)$ production and $\mathrm{Ca}^{2+}$ increase to result in vasoconstriction. $\mathrm{Ca}^{2+}$ and or second messenger $\mathrm{IP}_{3}$ diffusion to the $\mathrm{EC}$ through myoendothelial gap junctions (MEGJs) may lead to $\mathrm{Ca}^{2+}$

response in the $\mathrm{EC}$ to result in $\mathrm{Ca}^{2+}$ dependent vasodilatory feedback responses, including NO signaling to modulate the vessel tone (Dora, Doyle, and Duling 1997; Isakson, Ramos, and Duling 2007; Yashiro and Duling 2000; Kerr et al. 2012; Tran et al. 2012; Sandow et al. 2009). Different aspects of the NO mediated feedback response remains unclear and elucidation of the mechanisms regulating this process could provide novel insight into microvascular reactivity. 
Nitric oxide (NO) is produced in the endothelial cells (ECs), which line blood vessels, through the degradation of L-arginine by endothelial nitric oxide synthase (eNOS) enzyme. The activity of eNOS depends on the cytosolic EC calcium $\left(\mathrm{Ca}^{2+}\right)$ concentration (Busse and Mulsch 1990). The NO produced in the EC diffuses to the adjacent smooth muscle cell (SMC) to activate a cascade of signaling mechanisms including activation of soluble guanylate cyclase (sGC) to result in SMC relaxation. In the vasculature, $\mathrm{NO}$ have been identified as an endothelium derived relaxing factor (EDRF) and a key regulator of vascular tone and blood flow (Furchgott and Zawadzki 1980; Ignarro et al. 1987; Palmer, Ferrige, and Moncada 1987).

$\mathrm{EC}$ are in close proximity with the RBCs in the blood which are abundant in Hemoglobin $(\mathrm{Hb})$ concentrations. NO is scavenged rapidly through fast reactions with both oxy- and deoxy-Hb (Eich et al. 1996; Herold, Exner, and Nauser 2001) forming met- $\mathrm{Hb}\left(\mathrm{Hb}-\mathrm{Fe}^{3+}\right)$ and nitrate $\left(\mathrm{NO}_{3}{ }^{-}\right)$, which may severely limit $\mathrm{NO}$ diffusion and bioavailability of NO in the SMC. Several experimental studies and mathematical models have been carried out to examine how NO escapes scavenging through the complex interaction of NO with substances, such as heme-containing proteins and reactive oxygen species and are reviewed in (Tsoukias 2008; Buerk, Barbee, and Jaron 2011). Recent experimental studies on isolated vessels and vascular cell co-culture models in the absence of the blood revealed localized NO production through spatial localization of eNOS in the MP (Straub et al. 2012; Straub et al. 2011) as one of the safeguard for significant scavenging in the RBCs (Rahaman and Straub 2013; Gladwin and KimShapiro 2012) during the feedback. Straub et al. recently identified enriched expression of $\mathrm{Hb} \alpha$ at the MP (Straub et al. 2012) and revealed its role as a NO signaling switch 
(through scavenging of NO depending on its heme iron oxidation state) to control NO bioavailability and vascular tone during agonist induced stimulation (Straub et al. 2012; Rahaman and Straub 2013).

Mathematical modeling can assist in examination of NO pathway physiology and quantify vascular NO concentrations. Mass transport models for NO transport have been developed to examine its fate in the vasculature. We recently reported a comprehensive review of different mathematical models undertaken to describe NO transport in vasculature (Tsoukias 2008). We have previously developed coupled EC-SMC compartmental model integrated with NO signaling pathway to investigate the effect of myoendothelial communication on modulation of $\mathrm{SMC} \mathrm{Ca}^{2+}$ and membrane potential $\left(V_{\mathrm{m}}\right)$ dynamics. Very few theoretical models have examined the role of MP with spatially localized channels, proteins and molecules in the regulation of the vessel tone. We extended the coupled EC-SMC model compartmental model into a 2-D continuum model which incorporates accurate MP geometry from electron microscopy images and spatial localization of $\mathrm{IK}_{\mathrm{Ca}}$ and $\mathrm{IP}_{3} \mathrm{Rs}$ in the MP. This new formulation was utilized to investigate the role of localized signals in EC-SMC communication in absence of NO signaling (Nagaraja et al. 2013). Similarly, Brasen et al. have developed a 2-D axisymmetric model incorporating the anatomical structure of MPs into a two cell system (Brasen, Jacobsen, and Holstein-Rathlou 2012). Their results show that MPs may rectify the signal between the EC and SMC. Previous models did not examine the NO mediated feedback signaling during the stimulation of the SMC and its modulation by EC Hba. 
In this chapter, we present a computational model to examine the EC mediated NO signaling. Moreover we analyzed the relative contribution of enriched EC Hba in the MP and the RBCs in the modulation of NO feedback response. The NO signaling pathways was incorporated in the previously developed continuum EC-SMC model with MPs (Figure 3.1-A). The model accounts for preferential presence of the Hba, eNOS and $\mathrm{IP}_{3} \mathrm{Rs}$ in the MPs as suggested in experimental studies. We utilize this model to examine the contribution of NO feedback signaling in the regulation of vessel tone.
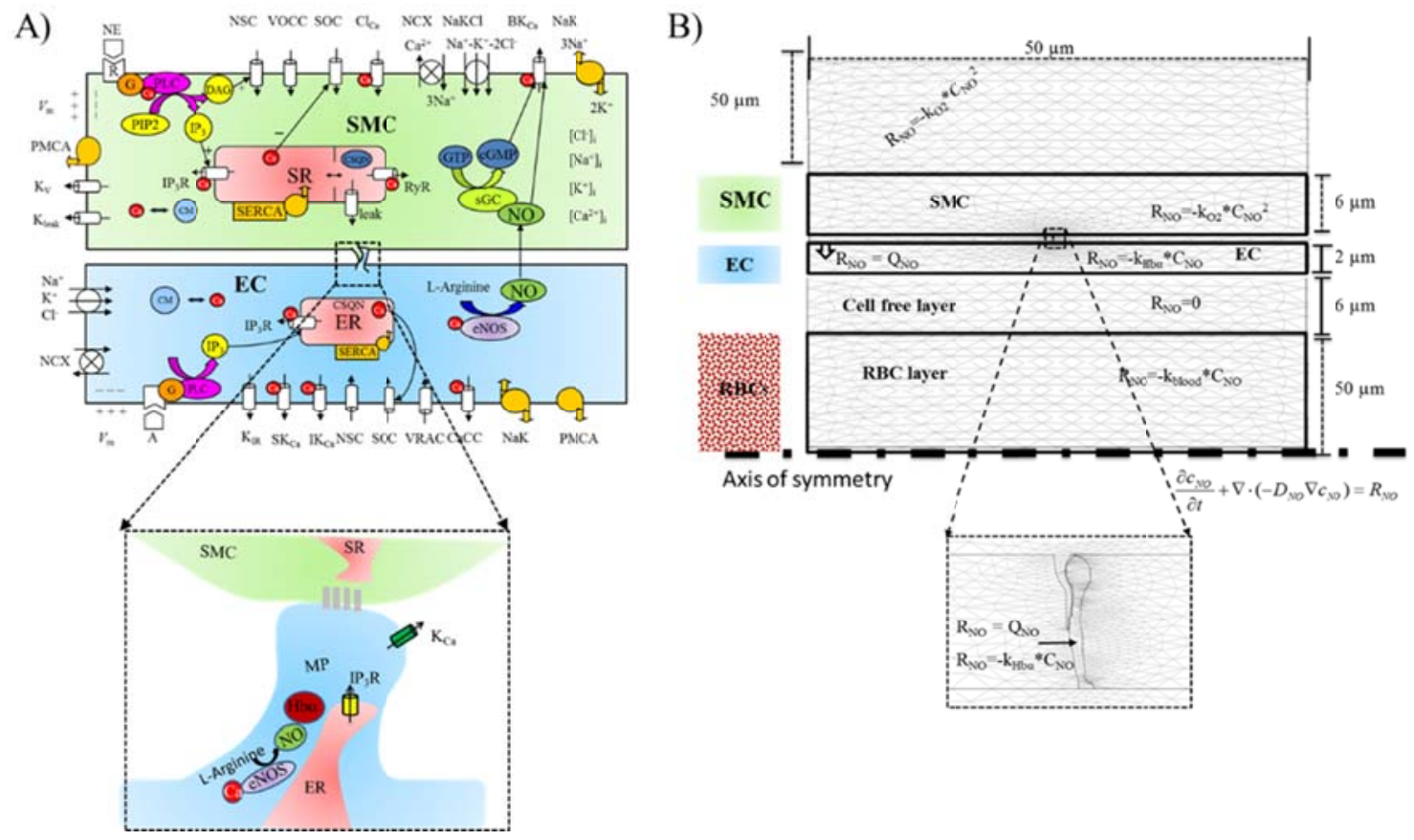

Figure 3.1 Schematic of the EC-SMC model, (A) Cartoon illustration describing all the channels, pumps and pathways considered in the model. (B) 2D axisymmetric model geometry with SMC, EC and RBC layer as rectangular domains. SMC coupled with EC with MP whose shape is imported from electron microscopy image by (Sandow et al. 2009) and MEGJs. Ion channel currents are uniformly distributed along the top and bottom boundaries of each cell. Inset contains zoomed view of the MP with mesh. eNOS, $\mathrm{IP}_{3} \mathrm{Rs}, \mathrm{K}_{\mathrm{Ca}}$ and $\mathrm{Hb} \alpha$ channels are localized in the MP. All other channels and pumps are also present in MP membrane, but proportionally to MP volume. Nitric oxide (NO) is produced in the EC and can react with $\mathrm{Hb} \alpha$ in the EC, diffuse into the lumen, where it reacts with hemoglobin $(\mathrm{Hb})$ of red blood cells (RBCs). It can also diffuse abluminally and react with oxygen or substrates such as soluble guanylate cyclase (sGC) in smooth muscle. 


\subsection{Methods}

\subsubsection{Continuum model}

We have previously developed detailed compartmental models of integrated $\mathrm{Ca}^{2+}$ and $V_{\mathrm{m}}$ dynamics in an isolated EC and SMC (Kapela, Bezerianos, and Tsoukias 2008; Silva, Kapela, and Tsoukias 2007), based primarily on data from RMA. The isolated models were combined into a two-cell (EC-SMC) and multicellular compartmental models to investigate the myoendothelial communication and conducted vasoreactivity respectively (Kapela, Bezerianos, and Tsoukias 2009; Kapela, Nagaraja, and Tsoukias 2010). We updated the compartmental models to capture the spatiotemporal nature of $\mathrm{Ca}^{2+}$ mobilization through the development of a continuum EC-SMC model, incorporated with the accurate MP geometry from experimental studies, and examined the role of MP in endothelial derived hyperpolarizing feedback response for the regulation of the vascular tone (Nagaraja et al. 2013), in the absence of nitric oxide (NO) signaling.

To examine the NO mediated myoendothelial feedback response for the regulation of vascular tone, and modulation of NO signaling by Hemoglobin $\alpha(\mathrm{Hb} \alpha)$ (which are recently reported to be expressed abundantly in MP (Straub et al. 2012)), we updated the model in (Nagaraja et al. 2013) to include NO signaling pathway. A layer representing the lumen of artery, which consist of RBCs that scavenge NO, and RBC free layer of 6 $\mu \mathrm{m}$ (Figure 3.1-A) between the EC and RBC layer were considered as in (Tsoukias, Kavdia, and Popel 2004). NO signaling pathway was implemented through introduction

of $\mathrm{Ca}^{2+}$ dependent NO generation (Kapela, Bezerianos, and Tsoukias 2009) in the EC and MP, NO diffusion across SMC, EC, cell free layer (cfl) and RBC layer (rbl), and NO scavenging. The EC, SMC, cfl and rbl were modeled as simplified rectangular domains 
with dimensions as shown in Figure 3.1-B. The model implemented only half of the EC and SMC by assuming symmetry for the other half. Majority of the $\mathrm{IK}_{\mathrm{Ca}}$ channels and around $10 \% \mathrm{IP}_{3} \mathrm{Rs}$ were localized in the MP as in (Nagaraja et al. 2013). Abundant expression of $\mathrm{Hb} \alpha$ in the MP and its co-localization with eNOS, reported in the recent experimental data (Straub et al. 2012), was tested with localization of range of $\mathrm{Hb} \alpha(0-1$ $\mu \mathrm{M}$ (The Hb $\alpha$ concentrations are reported based on the EC volume of $400 \mu \mathrm{m}^{3}$ throughout the manuscript)) and eNOS (0-100\% of the total eNOS) in MP in the model. All the other EC and SMC channels and pumps of the were uniformly distributed as in our previous model (Nagaraja et al. 2013).

\subsubsection{Ionic and $\mathrm{IP}_{3}$ transport}

The transport for individual ionic species is influenced by both electrical and concentration gradients, and was described using the Nernst-Planck electrodiffusion equation (Equation 3.1)

$$
\delta_{\text {buff }} \frac{\partial[S]}{\partial t}=\nabla .\left(D_{S} \nabla[S]+z_{s} F u_{m s}[S] \nabla V\right)-R_{s}
$$

\section{Equation 3.1}

where $S=\mathrm{Na}^{+}, \mathrm{K}^{+}, \mathrm{Cl}^{-}, \mathrm{Ca}^{2+}, D_{S}$ is the diffusion coefficient of ionic species $S, z_{S}$ is the valence of ionic species $S, \nabla V$ is the electrical gradient, $F$ is the Faraday constant and $u_{m S}$ is the ionic mobility given by $\frac{D_{S}}{R T}\left(\mathrm{R}\right.$ is the ideal gas constant $\left(8341 \mathrm{~mJ} \cdot \mathrm{mol}^{-1} \cdot \mathrm{K}^{-1}\right)$ and $\mathrm{T}$ is the absolute temperature). $R_{\mathrm{s}}$ is the source/sink term which includes the expressions for cytosolic $\mathrm{Ca}^{2+}$ exchange with the ER/SR, and $\mathrm{Ca}^{2+}$ buffering in the EC and MP. $\delta_{\text {buff }}$ accounts for the $\mathrm{Ca}^{2+}$ buffering in the SMC using a fast buffering approximation. Transport of $\mathrm{IP}_{3}$ and $\mathrm{Ca}^{2+}$ in ER/SR was described using Equation 3.2. 


$$
\delta_{b u f f} \frac{\partial[S]}{\partial t}=\nabla \cdot\left(D_{S} \nabla[S]\right)-R_{s}
$$

Equation 3.2

where $[S]$ is the concentration of either species ( $\mathrm{IP}_{3}, \mathrm{Ca}^{2+} \mathrm{ER}$ and $\left.\mathrm{SR}\right) . R_{s}$ is the source/sink term and includes $\mathrm{Ca}^{2+}$ exchange between stores and the cellular domains, and $\mathrm{IP}_{3}$ production and degradation. A uniform distribution of transmembrane channels and pumps was considered along the boundary of the cellular domains. The membrane currents were defined as boundary fluxes (Equation 3.3) across the top and bottom boundaries of EC, SMC and the MP boundaries.

$$
-n \cdot N_{S}=\frac{1}{z_{S} F} \sum_{K} I_{S, K}
$$

Equation 3.3

where $\mathrm{n}$ is the normal to the surface and $\mathrm{N}_{\mathrm{s}}$ is the membrane flux given by summation of all the transmembrane currents species for species $\mathrm{S}\left(I_{S, K}\right)$. The membrane currents were distributed between the MP and bulk cell according to their respective volumes. The membrane current definitions and parameters are identical to the original models (Kapela, Bezerianos, and Tsoukias 2008, 2009; Kapela and Tsoukias 2011; Silva, Kapela, and Tsoukias 2007).

\subsubsection{NO transport}

NO transport across the $\mathrm{EC}, \mathrm{SMC}$, cfl and rbl domains was implemented using unsteady reaction diffusion equation (Equation 3.4)

$$
\frac{\partial[N O]}{\partial t}=\nabla .\left(D_{N O} \nabla[N O]\right)-R_{N O, i}
$$

Equation 3.4 
where $[\mathrm{NO}]$ is the concentration of $\mathrm{NO}, D_{N O}$ is the diffusion coefficient of $\mathrm{NO}, \mathrm{R}_{\mathrm{NO}, \mathrm{i}}$ is the consumption rate of $\mathrm{NO}$ in each region and $\mathrm{i}=\mathrm{EC}, \mathrm{SMC}$, cfl and rbl. Continuity of concentration at the interface between adjacent layers was assumed. A zero concentration gradient boundary condition was imposed at the center of the arteriole considering axis of symmetry, and far from the SMC layer (50 $\mu \mathrm{m}$ away)

$$
\left.D_{N O} \frac{\partial[N O]}{\partial x}\right|_{x=0}=\left.D_{N O} \frac{\partial[N O]}{\partial x}\right|_{x=\infty}=0
$$

Equation 3.5

An effective first-order reaction rate mechanism (Equation 3.6) based on the previous NO transport model (Tsoukias, Kavdia, and Popel 2004) was used to describe the consumption rate of $\mathrm{NO}$ in $\mathrm{RBC}$ layer.

$$
R_{N O, r b l}=k_{\text {blood }}[N O]
$$

Equation 3.6

Consumption rate of NO in the EC and MP by Hba was implemented as a first order reaction rate directly proportional to concentration of $\mathrm{Hb} \alpha([\mathrm{Hb} \alpha])$ and $[\mathrm{NO}]$ in $\mathrm{EC}$ (Equation 3.7). In the control model all the Hba was localized in the projection.

$$
R_{N O, E C a n d M P}=k_{H b \alpha}[H b \alpha][N O]
$$

\section{Equation 3.7}

The consumption through reaction with oxygen was consider in SMC and cfl, and implemented through a second order reaction rate proportional to concentration of oxygen $\left(\left[\mathrm{O}_{2}\right]\right)$ and square of $[\mathrm{NO}]$ (Equation 3.8) as described in (Lewis and Deen 1994).

$$
R_{N O, S M C \& c f l}=4 k_{1}\left[O_{2}\right][N O]^{2}
$$

Equation 3.8 
$\mathrm{EC} \mathrm{Ca}^{2+}$ dependent NO production by endothelial nitric oxide synthases (eNOS) enzyme was implemented using (Equation 3.9) as in our previous model (Kapela, Bezerianos, and Tsoukias 2009), and incorporated in the EC and MP domains.

$$
Q_{\mathrm{NO}}=Q_{\mathrm{NO}, \max } \frac{\left[\mathrm{Ca}^{2+}\right]^{4.2}}{\left[\mathrm{Ca}^{2+}\right]^{4.2}+k_{m, C a}^{\mathrm{NO}} 4.2}
$$

Equation 3.9

The flux was distributed between the MP and bulk cell according to their respective volumes. A significant percentage ( $30 \%$ under control conditions) of the total eNOS was localized in the MP and remaining distributed in the bulk EC. The distribution of the eNOS was controlled by the maximum NO flux parameter $\left(Q_{N O, \max }\right.$-Table 3.1).

Table 3.1 Parameter values

\begin{tabular}{|l|l|l|l|}
\hline Parameters & Value & Description & REF \\
\hline$D_{N O}\left(\mathrm{~cm}^{2} / \mathrm{s}\right)$ & $3.3 \times 10^{-5}$ & Diffusion coefficient of NO & $\begin{array}{l}\text { (Tsoukias, } \\
\text { Kavdia, and } \\
\text { Popel 2004) }\end{array}$ \\
\hline$k_{\text {blood }}\left(\mathrm{s}^{-1}\right)$ & 6400 & Blood reaction constant & $\begin{array}{l}\text { (Tsoukias and } \\
\text { Popel 2002) }\end{array}$ \\
\hline$k_{H b}\left(\mu \mathrm{M}^{-1} \mathrm{~s}^{-1}\right)$ & 100 & NO-oxyHb reaction constant & $\begin{array}{l}\text { (Tsoukias, } \\
\text { Kavdia, and } \\
\text { Popel 2004) }\end{array}$ \\
\hline$k_{l}\left(\mathrm{M}^{-2} \mathrm{~s}^{-1}\right)$ & $2.1 \times 10^{6}$ & $\mathrm{NO}^{-\mathrm{O}_{2} \text { reaction constant }}$ & $\begin{array}{l}\text { (Lewis and Deen } \\
\text { 1994) }\end{array}$ \\
\hline$[\mathrm{Hb \alpha}](\mu \mathrm{M})$ & $0.5(0-1)$ & Hba concentration in EC & Assumed \\
\hline$\left[\mathrm{O}_{2}\right](\mu \mathrm{M})$ & 277 & $\mathrm{O}_{2}$ concentration in SMC and cfl & \\
\hline $\begin{array}{l}Q_{N O, m a x} \\
\left(\mathrm{nmolcm}{ }^{-2} \mathrm{~s}^{-1}\right)\end{array}$ & $2.65 \times 10^{-3}$ & $\begin{array}{l}\text { Maximum NO production rate } \\
\left(\text { equivalent to 53 } \mu \mathrm{Ms} \mathrm{s}^{-1} \text { (assuming }\right. \\
\text { EC thickness of 0.5 } \mu \mathrm{m})\end{array}$ & $\begin{array}{l}\text { (Tsoukias, } \\
\text { Kavdia, and } \\
\text { Popel 2004; } \\
\text { Chen, Pittman, } \\
\text { and Popel 2008; } \\
\text { Tsoukias 2008) }\end{array}$ \\
\hline $\begin{array}{l}N O \\
K_{m, c a}(\mathrm{nM})\end{array}$ & 300 & $\begin{array}{l}\text { Ca } \\
\text { maximum NO release rate. }\end{array}$ & $\begin{array}{l}\text { Kapela, } \\
\text { Bezerianos, and } \\
\text { Tsoukias 2009) }\end{array}$ \\
\hline
\end{tabular}


NO-cGMP pathway (Figure 3.1-B) and its modulation of large-conductance $\mathrm{Ca}^{2+}$ activated potassium channels in the SMC was implemented as in our previous model (Kapela, Bezerianos, and Tsoukias 2009). The parameter values used in the model are provided in Table 3.1 .

\subsection{Results}

\subsubsection{No profiles arising from agonist stimulation of the EC and SMC}

Agonist stimulation of the EC with Ach (1 a.u.- $\mathrm{IP}_{3}$ production rate: $5.5 \times 10^{-8} \mathrm{M} / \mathrm{s}$ ) increased $\mathrm{IP}_{3}$ concentrations in the EC bulk and MP (Figure 3.2-A, red and green dotted lines), causing store $\mathrm{Ca}^{2+}$ release to elevate global and local $\mathrm{EC} \mathrm{Ca}^{2+}$ concentrations (Figure 3.2-A, red and green solid lines), similar to our previous model (Nagaraja et al. 2013).
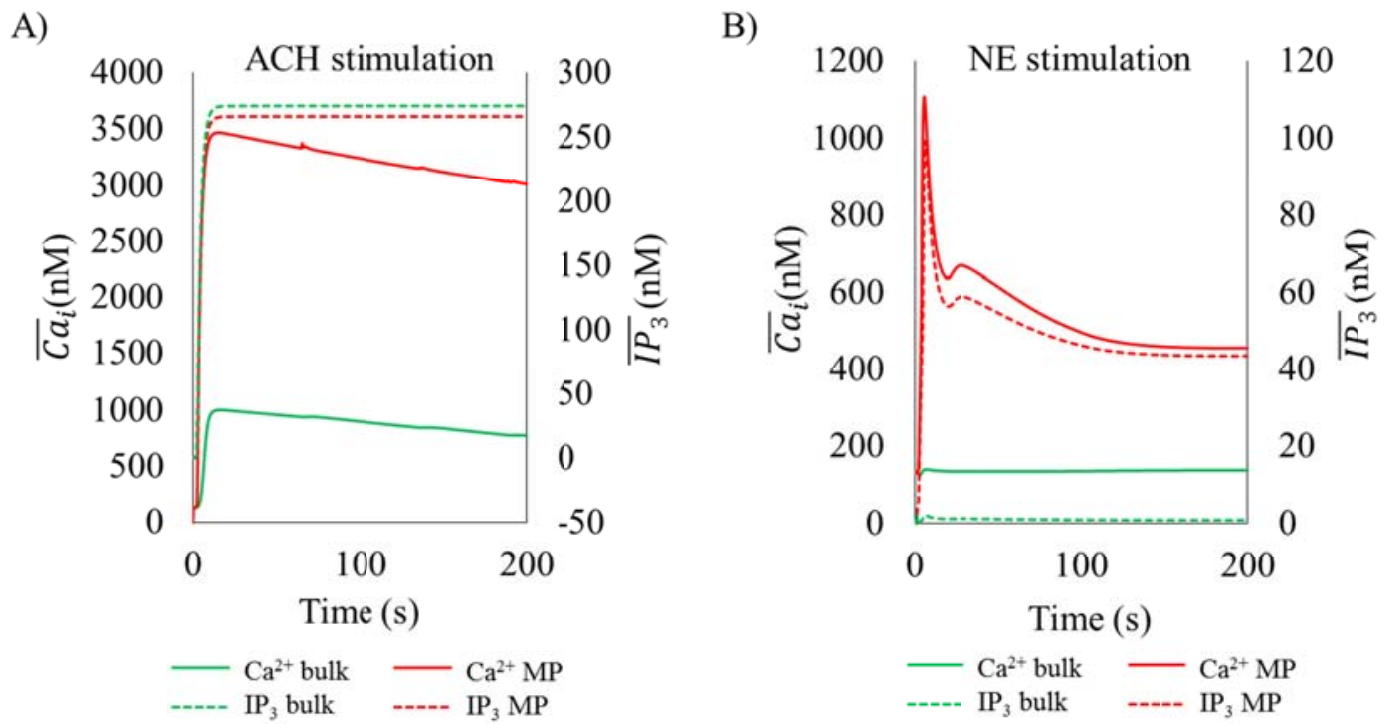

Figure 3.2 $\mathrm{Ca}^{2+}$ (continuous lines) and $\mathrm{IP}_{3}$ (dashed line) concentrations in the EC MP (red lines) and EC bulk (green lines) during (A) NE stimulation of SMC and (B).ACh stimulation of EC 
This resulted in the production of NO through $\mathrm{Ca}^{2+}$ dependent activation of eNOS. The resulting near steady state spatial NO profiles in the EC and SMC with uniform distribution of eNOS, in the presence and absence of uniformly distributed $0.5 \mu \mathrm{M} \mathrm{Hb} \alpha$ in the EC, are depicted color-coded and shown in Figure 3.3-A and B respectively. Illustrative spatial profiles near steady state with $100 \%$ localized eNOS in the presence and absence of $\mathrm{Hb} \alpha$ are shown in Figure 3.3-C and D.

A)
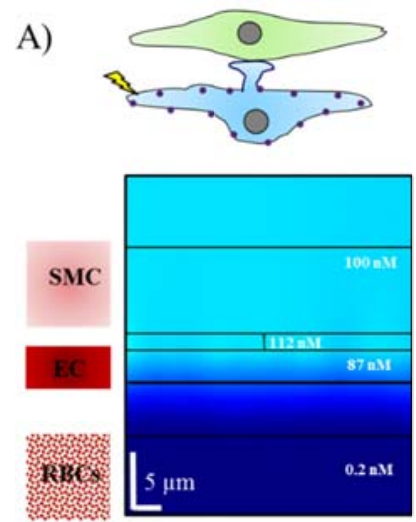

C)
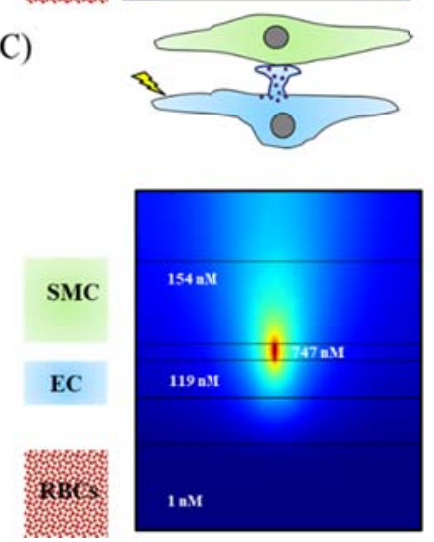

B)
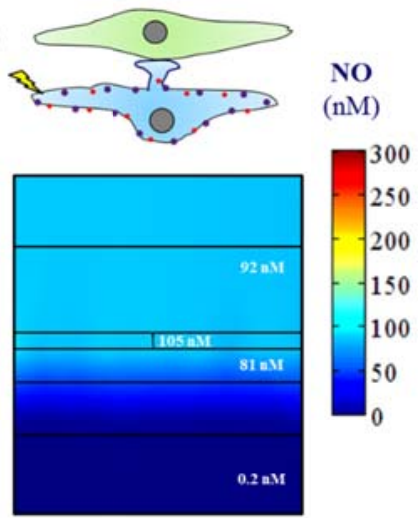

D)
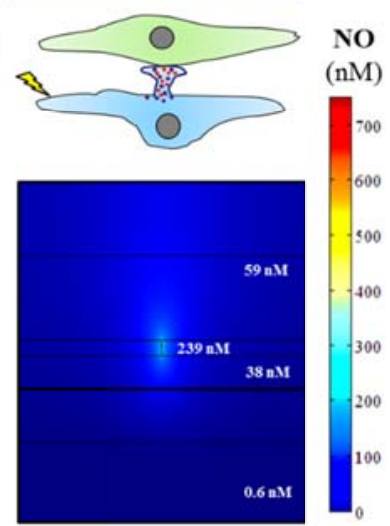

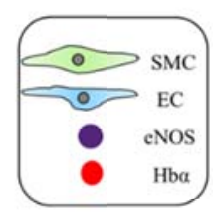

Figure 3.3 Illustrative spatial NO profiles during EC stimulation. (A) Uniform eNOS, Hba (-), (B) Uniform eNOS, Uniform $\mathrm{Hb} \alpha(0.5 \mu \mathrm{M})$, (C) Localized eNOS, Hb $\alpha$ (-), and (D) Localized eNOS, Localized $\mathrm{Hb} \alpha(0.5 \mu \mathrm{M})$.

Significant amount of NO produced in the EC was scavenged in the RBC layer. The rest of NO diffuses towards across the SMC to result in a steady state average NO concentration of 92-100 nM (Figure 3.4) in the SMC with uniformly distributed eNOS, 
similar to those observed in the previous 1-D model of NO transport in arterioles (Tsoukias, Kavdia, and Popel 2004). Localization of 30 and 100\% eNOS in the MP in the absence of $\mathrm{Hb} \alpha$ resulted in increased steady state $\mathrm{NO}$ levels experienced by the SMC to around 124 and $154 \mathrm{nM}$ respectively (Figure 3.4). Localized $\mathrm{Hb} \alpha(0.5 \mu \mathrm{M}$ (concentration is per EC volume)) reduced the SMC NO concentrations to around 59 and $89 \mathrm{nM}$ respcetively (Figure 3.4) in the presence of localized $\mathrm{Hb} \alpha(0.5 \mu \mathrm{M}$ (concentration is per EC volume)) in the MP.

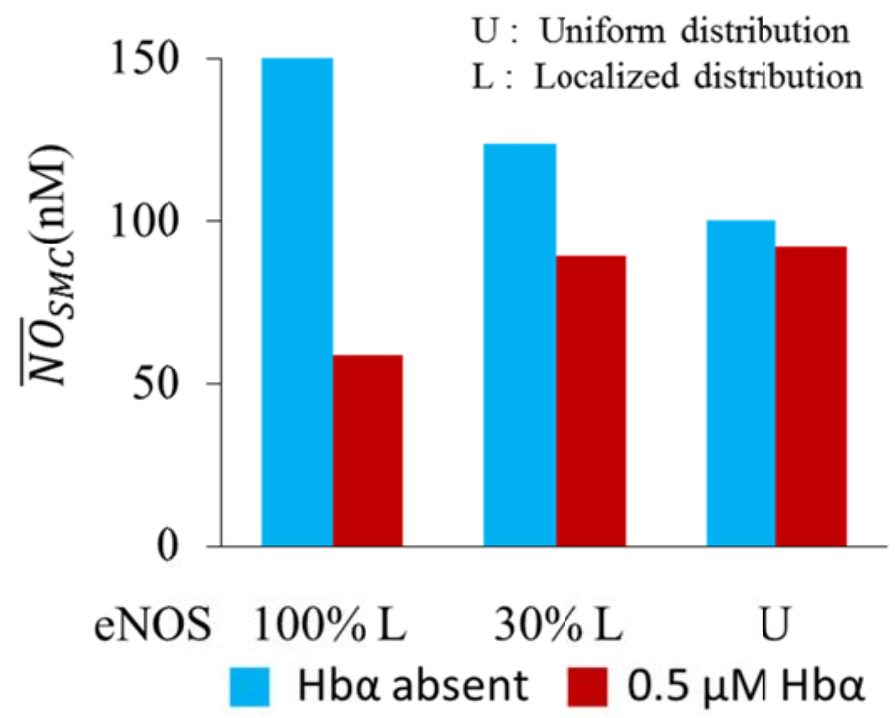

Figure 3.4 Average SMC NO concentration during EC stimulation in presence of uniform eNOS, and localized eNOS ( $30 \%$ and $100 \%$ of total eNOS) in the MP. Blue bar represent concentration in the absence of $\mathrm{Hb} \alpha$ and red bars are in the presence of $\mathrm{Hb} \alpha(0.5 \mu \mathrm{M})$.

Norepinephrine $(\mathrm{NE})(1 \mu \mathrm{M})$ stimulation of SMC in the model resulted in large amplitude $(\mu \mathrm{M}) \mathrm{Ca}^{2+}$ (Figure 3.2-B, red solid line) and $\mathrm{IP}_{3}$ transient (Figure 3.2-B, red dotted line) in EC MP arising mediated by intercellular $\mathrm{IP}_{3}$ diffusion from the $\mathrm{SMC}$ to the EC, similar to our previous model (Nagaraja et al. 2013). The EC bulk $\mathrm{IP}_{3}$ and $\mathrm{Ca}^{2+}$ concentration did not increase during NE stimulation (Figure 3.2-B, green solid and dotted lines). Illustrative steady spatial profiles of NO concentration obtained during the 
stimulation of SMC are depicted color-coded and shown in Figure 3.5. Uniformly

distributed eNOS in the EC yielded insignificant NO levels in the EC (Figure 3.5-A) due to lack of increase in global $\mathrm{EC} \mathrm{Ca}^{2+}$ concentrations. Localization of eNOS (100\%)

(Figure 3.5-B) in the MP produced sustained NO production in the MP which spreads across the SMC on abluminal side and scavenged in the RBC layer on the luminal side.

Presence of uniformly distributed $\mathrm{Hb} \alpha(0.5 \mu \mathrm{M})$ in the EC and the MP attenuated slightly the amplitude of NO levels produced the in MP through consumption by Hba as shown in Figure 3.5-C. Localization of $100 \% \mathrm{Hb} \alpha(0.5 \mu \mathrm{M})$ in the MP further reduced the NO levels through higher consumptions by $\mathrm{Hb} \alpha$ (Figure 3.5-D) due to its close proximity with eNOS in the MP.

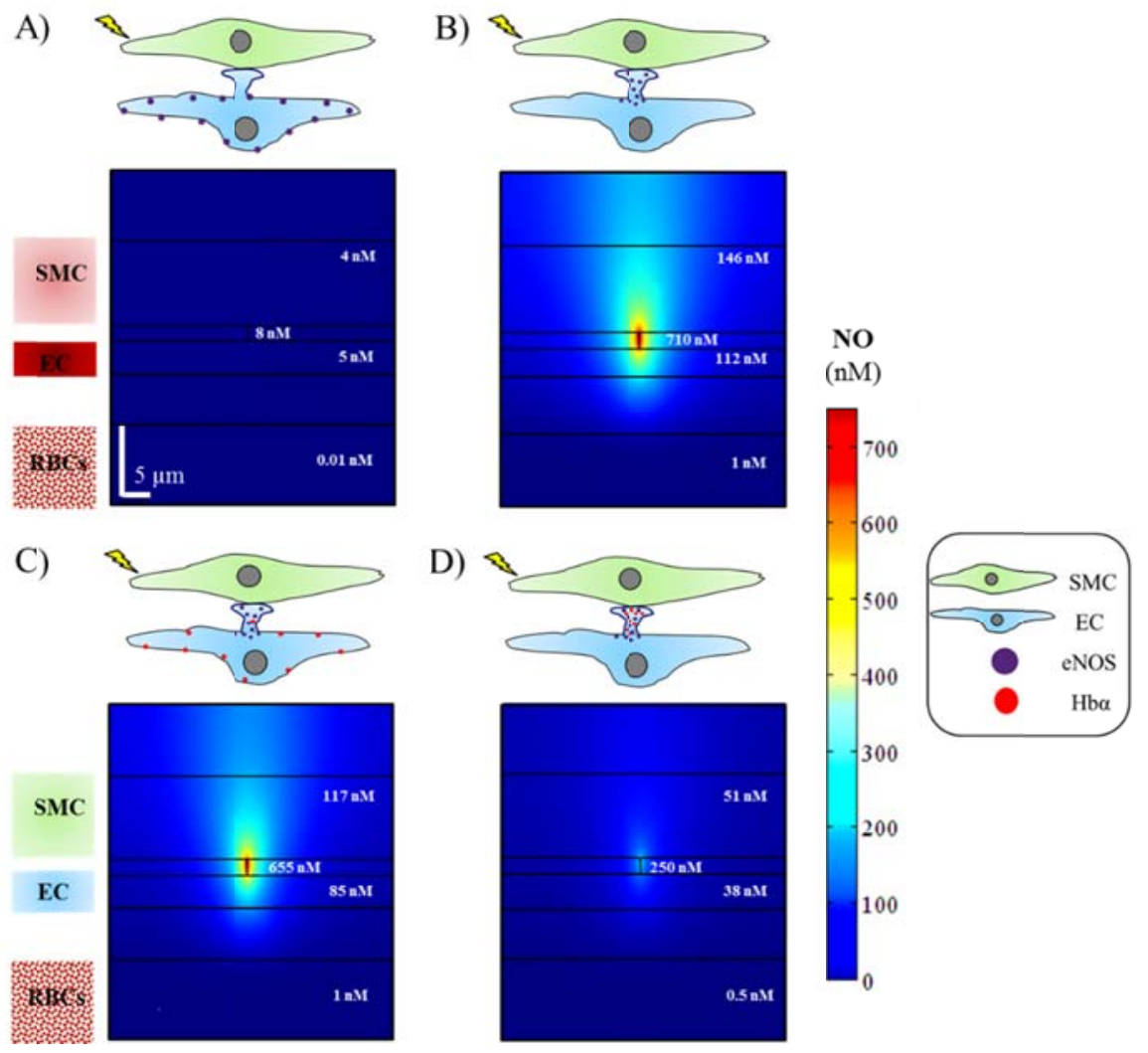

Figure 3.5 Illustrative spatial NO profiles during SMC stimulation. (A) Uniform eNOS, Hba (-), (B) Localized eNOS, $\mathrm{Hb} \alpha(-)$, (C) Localized eNOS, Uniform $\mathrm{Hb} \alpha(0.5 \mu \mathrm{M})$, and (D) Localized eNOS, Localized $\mathrm{Hb} \alpha(0.5 \mu \mathrm{M})$ 


\subsection{2 eNOS localization during SMC stimulation}

eNOS have been shown to enriched and active in the MPs (Straub et al. 2011), but their density and activation characteristics remain unclear and it is likely that they vary across different vascular beds. Average SMC NO concentrations arising from NE stimulation of the SMC for $200 \mathrm{sec}$ (near steady state) were observed in the model. Lack of increase in the global $\mathrm{Ca}^{2+}$ levels during NE stimulation of SMC resulted in insignificant steady state average NO levels of around $5 \mathrm{nM}$ in the SMC (Figure 3.6-A, red bar), in the presence of uniformly distributed eNOS in the EC. Hence, eNOS localization in the MP may be necessary for generation of any significant NO mediated myoendothelial feedback. Localized $\mathrm{Ca}^{2+}$ mobilization in the MP arising from NE stimulation of SMC in the model, yielded significantly higher SMC NO concentrations in range of 50-150 nM (Figure 3.6-A) in presence of 30-100\% eNOS localized in the MP, in the absence of $\mathrm{Hb} \alpha$. A control value of $30 \%$ eNOS distribution in the MP was considered in the model.
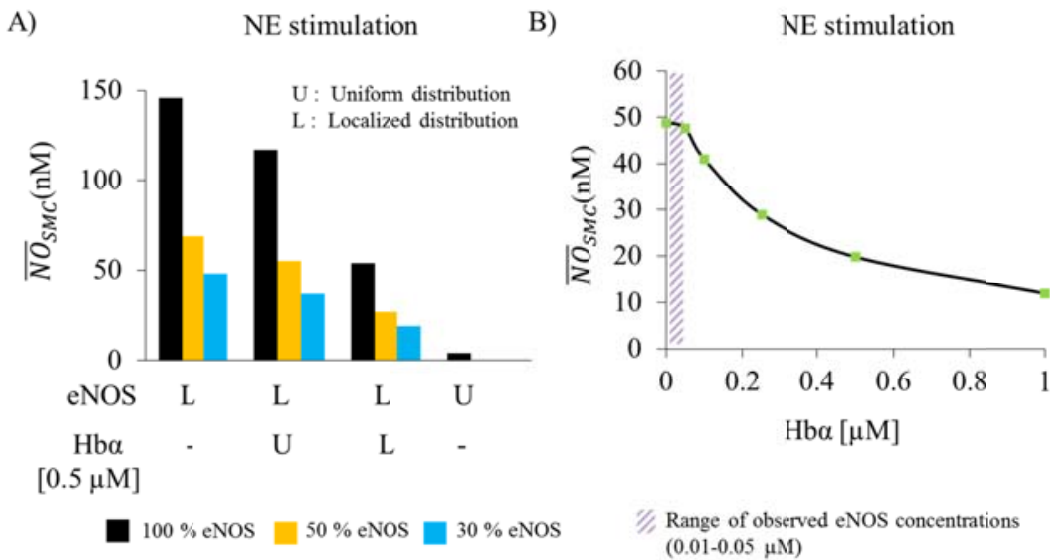

Figure 3.6 Average SMC NO concentration observed during SMC stimulation, in the presence of (A) uniform (U) distributed eNOS in the EC or localized (L) eNOS distribution in the MP(30\% (blue bar), $50 \%$ (orange bar) and 100\% (black bar) of total eNOS) and in the absence (-) of Hbo or presence of $0.5 \mu \mathrm{M} \mathrm{Hb} \alpha$ distributed uniformly (U) or localized (L).in the MP (B) 30\% eNOS localized in the MP and range of $\mathrm{Hb} \alpha$ $(0-1 \mu \mathrm{M})$ localized in the MP 


\subsubsection{Hba localization during SMC stimulation}

Enriched Hba expression in the MP have been reported to regulate of eNOS mediated control of vascular reactivitiy through NO consumption (Straub et al. 2012). We analyzed

a range of Hb $\alpha$ concentration $\left(0-1 \mu \mathrm{M}\right.$ (concentrations per EC volume of $\left.400 \mu \mathrm{m}^{3}\right)$ ) through their localization in the MP, to observe its effect on the available NO concentrations in the SMC. The initial range of $\mathrm{Hb} \alpha$ concentration was chosen on the basis of reported 0.01 to $0.04 \mu \mathrm{M}$ eNOS concentration (Figure 3.6-B, purple bar) in the EC but had no significant effect on the NO levels in the SMC during NE stimulation (Figure 3.5-B). Increased concentrations of $\mathrm{Hb} \alpha$ between $0.05 \mu \mathrm{M}-1 \mu \mathrm{M}$ localized in the MP resulted in exponential decrease in the mean NO concentrations generated in the SMC. $0.5 \mu \mathrm{M} \mathrm{Hb} \alpha$ resulted in reduction of mean NO levels in the SMC during NE stimulation by more than $60 \%$ (Figure 3.6-A and B), for varied eNOS localization (30$100 \%$ of total eNOS), and was considered as the control value in the model. Uniformly distributed $0.5 \mu \mathrm{M} \mathrm{Hb} \alpha$ in the EC had no significant modulation on the SMC NO concentrations (less than $20 \%$ change (Figure 3.6-A)).

\subsubsection{SMC $V_{m}$ feedback}

Under the control conditions (localized $\mathrm{IP}_{3} \mathrm{Rs}, \mathrm{IK}_{\mathrm{Ca}}$ eNOS and $\mathrm{Hb} \alpha$ in the MP), NE stimulation of the SMC for $200 \mathrm{~s}$, resulted in the SMC depolarization to about $-48 \mathrm{mV}$ (Figure 3.7-A, green solid line). Block of the NO signaling pathway in the control model reduced the myoendothelial feedback and enhanced SMC depolarization to about $-46 \mathrm{mV}$ (Figure 3.7-A, purple dashed-dotted line). The absence of $\mathrm{Hb} \alpha$ (zero concentration in the model) enhanced NO mediated myoendothelial feedback to result in reduction of SMC depolarization to around $-50 \mathrm{mV}$ (Figure 3.7-A, red dotted line), bringing it close to the 
resting SMC $V_{m}$ levels. Lack EC mediated $\mathrm{NO}$ signaling and hyperpolarization (EDH), resulted in enhanced SMC depolarization to around $-43.5 \mathrm{mV}$ (Figure 3.7-A, orange dashed line). This was implemented through the block of $\mathrm{IP}_{3}$ diffusion from the SMC to the EC which is the major signaling molecule generating significant $\mathrm{Ca}^{2+}$ transient in the MP during SMC stimulation in the model as shown in our previous study (Nagaraja et al. 2013). Lack of the MP $\mathrm{Ca}^{2+}$ transient resulted in insignificant NO production and inactivity of the $\mathrm{EC} \mathrm{IK} C \mathrm{Ca}$ and $\mathrm{SK}_{\mathrm{Ca}}$ channels (data not shown).

A)

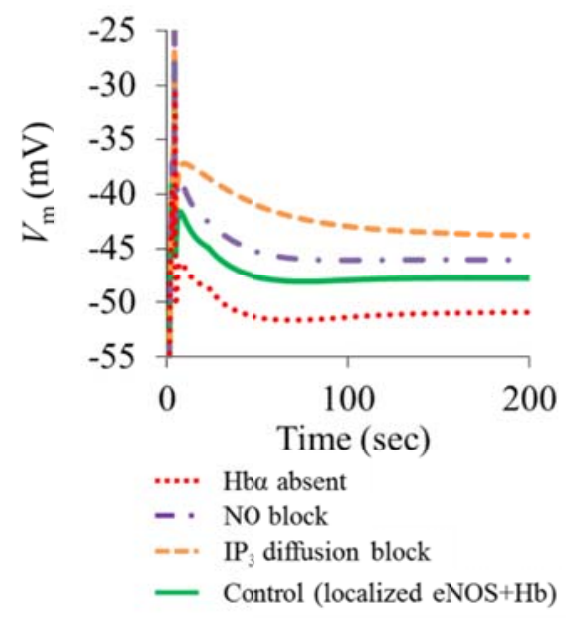

B)

NE stimulation

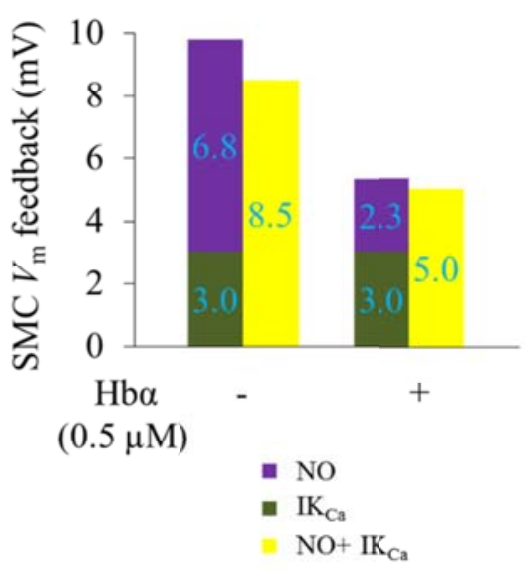

Figure 3.7 SMC hyperpolarization during SMC stimulation in presence of spatially enriched eNOS in the.MP (30\% of total eNOS), (A) under control conditions (green continuous line), block of NO signaling (purple dashed dotted line), absence of $\mathrm{Hb} \alpha$ (red dotted line) and block of $\mathrm{K}_{\mathrm{ca}}$ channels and NO signaling (orange dashed line). (B) Amount of feedback from NO mediated signaling (purple bar) during $\mathrm{K}_{\mathrm{Ca}}$ block, activation of $\mathrm{K}_{\mathrm{Ca}}$ channels during $\mathrm{NO}$ signaling block (green bar) and presence of both $\mathrm{NO}$ and $\mathrm{K}_{\mathrm{Ca}}$ mediated hyperpolarization.

We summarized the NO and EDH mediated feedback observed in the model in the presence or absence of localized Hb $\alpha$ in the MP (Figure 3.7-B). We observed around 3 $\mathrm{mV}$ feedback through activation of the $\mathrm{IK}_{\mathrm{Ca}}$ channels, in the absence of $\mathrm{NO}$ signaling, similar to our previous model (Nagaraja et al. 2013) in both the presence and absence of localized $\mathrm{Hb} \alpha$ (Figure 3.7-B, Green bars). NO signaling pathway, in the absence of $\mathrm{IK}_{\mathrm{Ca}}$ 
mediated hyperpolarization and localized $\mathrm{Hb} \alpha$, resulted in a feedback of around $6.5 \mathrm{mV}$ which was significantly diminished (to around $\sim 2 \mathrm{mV}$ ) in the presence of localized $\mathrm{Hb} \alpha$ in the MP (Figure 3.7-B, purple bars). Localized $\mathrm{Hb} \alpha$ reduced the feedback (of both $\mathrm{IK}_{\mathrm{Ca}}$ mediated hyperpolarization and $\mathrm{NO}$ signaling) from about $8 \mathrm{mV}$ to $5 \mathrm{mV}$ in the model (Figure 3.7-B, yellow bars).

\subsubsection{Erythrocyte versus EC Hba NO consumption}

$\mathrm{Hb} \alpha$ modulation of NO bioavailability have been recently reported in isolated vessels in the absence of NO consumption by erythrocytes (Straub et al. 2012). We examined the relative contribution of erythrocyte in the lumen which are distant (about $6 \mu \mathrm{m}$ ) from the site of NO production, and $\mathrm{Hb} \alpha$ colocalized with eNOS in the consumption of the NO (Figure 3.8). The absence of both erythrocyte $\left(k_{b l o o d}=0\right)$ and EC $\mathrm{Hb} \alpha([\mathrm{Hb} \alpha]=0)$ resulted in mean NO concentrations in the SMC around 8 and $11 \mu \mathrm{M}$ during SMC and EC stimulation respectively suggesting a slightly diminished NO production during SMC stimulation, occurring in the model from the lack of $\mathrm{EC} \mathrm{Ca}^{2+}$ bulk increase. The NO concentrations took a long time ( $\sim 60 \mathrm{sec})$ to approach steady state in the presence of only NO consumption being reaction with oxygen in the model (Figure 3.8-A and B, red dotted line). In the absence of erythrocytes consumption, localized $\mathrm{Hb} \alpha(0.5 \mu \mathrm{M})$ provided a significant reduction in the steady state mean NO concentration in the SMC to around $650 \mathrm{nM}(\sim 6 \%$ of $11 \mu \mathrm{M})$ and $120 \mathrm{nM}(\sim 1.5 \%$ of $8 \mu \mathrm{M})$ ((Figure 3.8-A and B ,yellow dashed line) for the agonist stimulation EC and SMC respectively suggesting enhanced $\mathrm{Hb} \alpha$ consumption during feedback. Although not intuitive at the first, consumption through erythrocytes which are located away from NO production site, in the absence of localized $\mathrm{Hb} \alpha$, resulted in 2-fold (to around $50 \mathrm{nM}$ ) and 5-fold higher (to 
around $120 \mathrm{nM}$ ) reduction in mean SMC NO concentrations than observed through consumption from only localized $\mathrm{Hb} \alpha$ in the absence of erthrocytes (Figure 3.8-A and B, orange dashed-dotted line) during SMC and EC stimulation respectively. Analytical calculations using mass balance equation also revealed 5 fold higher NO consumption from the erythrocyte layer than from the EC Hba similar to that observed in the model (see supplement). In the control model (30\% eNOS localized in the MP) in the presence of both erythrocyte and localized $\mathrm{Hb} \alpha(0.5 \mu \mathrm{M}$ in the MP), agonist stimulation of the EC and SMC resulted in the mean NO concentrations in the SMC of $81 \mathrm{nM}$ and $17 \mathrm{nM}$ respectively (Figure 3.8-A and B, green solid line). Reduced mean SMC NO concentration during the SMC stimulation comparted to EC stimulation was from the lack of NO production in the bulk EC in the absence of $\mathrm{EC}$ bulk $\mathrm{Ca}^{2+}$ increase and enhanced $\mathrm{Hb} \alpha$ scavenging of the locally produced NO during NE stimulation.

To further analyze the effect of $\mathrm{Hb} \alpha$ scavenging, we reported the effect of NO consumption from a rage of $\mathrm{Hb} \alpha$ concentration localized in the MP both in the presence and absence of $\mathrm{NO}$ consumption in the RBC layer. In the absence of erythrocyte consumption, $\mathrm{Hb} \alpha$ concentration comparable to observed eNOS concentration (Figure 3.8-C, purple bar) resulted in significant drop in the mean NO concentration in the SMC to $16 \%$ and $30 \%$ of the $\mathrm{NO}$ levels in the absence of $\mathrm{Hb} \alpha$ during the stimulation of SMC and EC cell respectively (Figure 3.8-C, black and blue dotted lines). Higher Hba concentration reduced further the NO levels. However, in the presence of erythrocyte consumption $\mathrm{Hb} \alpha$ concentration in the range $(0.01$ to $0.05 \mu \mathrm{M})$ had no significant modulation of SMC NO levels for both the stimulation of EC and SMC cell. 50 to 100 fold higher concentrations than experimentally observed eNOS concentrations were 
essential to reduce the SMC NO by more than $50 \%$ from its levels observed in the absence of $\mathrm{Hb} \alpha$ (Figure 3.8-C, black and blue solid lines). Moreover we observed enhanced scavenging of locally produced NO by Hba during feedback.

A)

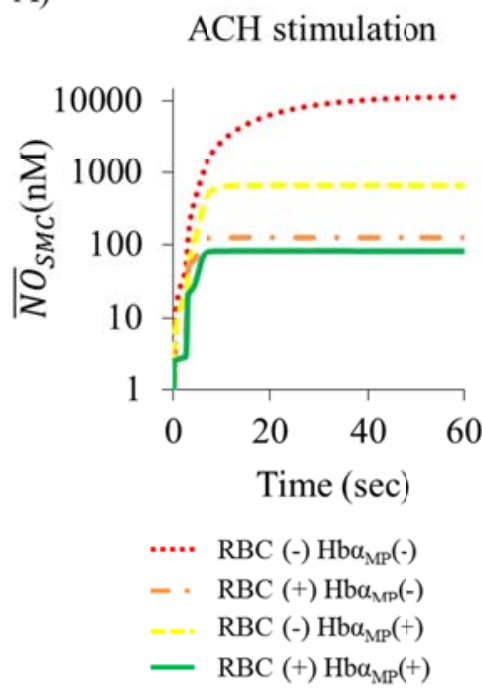

B)

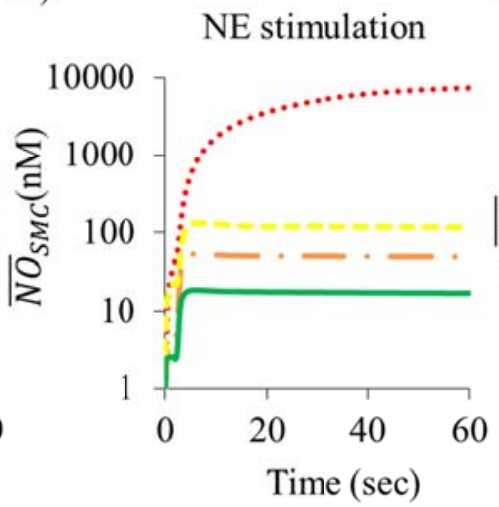

… $\mathrm{RBC}(-) \mathrm{Hb} \alpha_{\mathrm{MP}}(-)$

- RBC (+) $\mathrm{Hb} \alpha_{\mathrm{MP}}(-)$ $\mathrm{RBC}(-) \mathrm{Hb}_{\mathrm{MP}}(+)$

$-\mathrm{RBC}(+) \mathrm{Hb}_{\mathrm{MP}}(+)$
C)

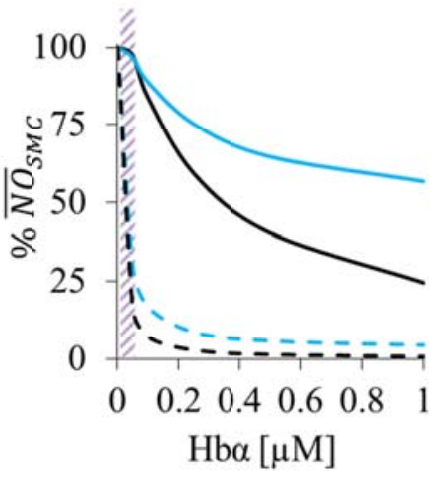

- Ach stim $\mathrm{RBC}(+)$

$=$ Ach stim RBC(-)

- NE stim RBC (+)

--- NE stim RBC (-)

Region of observed eNOS concentrations in EC

Figure 3.8 Erythrocyte versus EC Hb $\alpha$ regulation of mean SMC NO levels, (A) after $200 \mathrm{~s}$ stimulation of the EC, (B) after $200 \mathrm{~s}$ stimulation of the SMC. (C) Percentage change in mean NO levels in the SMC with increase in localized $\mathrm{Hb} \alpha$ concentration in the MP during the presence of NO scavenging in the RBC layer (blue lines) or absence of $\mathrm{NO}$ scavenging in the RBC layer (black lines).

\subsection{Discussion}

The primary aim of the study was to examine the EC cell generated NO signaling and its modulation during agonist stimulation of vascular cells. Using the 2-D continuum model with enriched localization of eNOS and $\mathrm{Hb} \alpha$ in the MP, we examined $\mathrm{EC} \mathrm{Ca}^{2+}$ mediated NO signaling and its regulation of $\mathrm{SMC} \mathrm{Ca}^{2+}$ and $V_{\mathrm{m}}$ alterations during contractile stimuli. Simulations showed significant feedback (few $\mathrm{mV}$ ) arising from NO signaling and its modulation through localized $\mathrm{Hb} \alpha$ and the RBCs. 
NO mediated regulation of vascular tone is attributed to an increase in $\mathrm{EC} \mathrm{Ca}^{2+}$ concentrations. We observed $\mathrm{IP}_{3}$ and $\mathrm{Ca}^{2+}$ increase in the $\mathrm{EC}$ (Figure 3.2-B) confined to the MPs as in our previous model (Nagaraja et al. 2013) during SMC stimulation, consistent with recent experimental evidence of local $\mathrm{EC} \mathrm{Ca}^{2+}$ events in and around $\mathrm{EC}$ MPs following SMC stimulation (Tran et al. 2012; Kansui, Garland, and Dora 2008). Our previous modeling efforts and theoretical estimations predict suggest that passive diffusion of $\mathrm{Ca}^{2+}$ and/or $\mathrm{IP}_{3}$ into $\mathrm{EC}$ is insufficient for a global $\mathrm{Ca}^{2+}$ mobilization (Nagaraja et al. 2013; Nagaraja, Kapela, and Tsoukias 2012). Significantly smaller surface area and volume of the MP with respect to the bulk of EC, restricted diffusivity and spatial polarization of store receptors (RyRs and/or $\mathrm{IP}_{3} \mathrm{Rs}$ ) around the MP can amplify the weak fluxes of $\mathrm{Ca}^{2+}$ and $\mathrm{IP}_{3}$ to induce local $\mathrm{Ca}^{2+}$ transients (Ledoux et al. 2008; Nagaraja et al. 2013; Isakson 2008).

In the model the absence of significant $\mathrm{EC}$ bulk $\mathrm{Ca}^{2+}$ increase during SMC stimulation necessitates spatial localization of eNOS around the MP for NO production through $\mathrm{Ca}^{2+}$ dependent eNOS activation. Uniformly distributed eNOS in the EC resulted in insignificant NO production. Increased localized NO production with rise in the amount of eNOS localized in the MP was observed in the model (Figure 3.5 and Figure 3.6-A). The locally produced NO diffused to the adjacent SMC in the model to provide few $\mathrm{mV}$ feedback through the NO-cGMP pathway (Figure 3.7-A and B). Spatially enriched eNOS in specific cellular regions resulting in local NO production have been observed experimentally (Qian et al. 2010; Iwakiri et al. 2006). Recent experimental studies revealed abundant expression of eNOS in the MP and suggest paracrine release of localized NO in providing feedback SMC constriction (Straub et al. 2011). 
Several mechanisms have been proposed to account for a more efficient delivery of endothelium-derived $\mathrm{NO}$ to the $\mathrm{SMC}$ and for $\mathrm{NO}$ preservation and reduced scavenging by the hemoglobin in the blood (Buerk, Barbee, and Jaron 2011; Tsoukias 2008).

Localized NO production at the EC-SMC junction (i.e. near the MP) has been suggested as one of the possible mechanism for reduced NO scavenging in the blood (Gladwin and Kim-Shapiro 2012; Rahaman and Straub 2013; Straub et al. 2012). In the model local NO production during EC stimulation from spatially enriched eNOS around the MP increased mean SMC NO concentration by $25-50 \mathrm{nM}$ ( from uniform NO production) in the presence of $\mathrm{Hb}$ consumption in the $\mathrm{RBC}$ layer (Figure 3.4) suggesting reduced scavenging of the locally produced NO . Moreover, local NO production during SMC stimulation increased mean SMC concentration by 50-140 nM (Figure 3.5) which can be ascribed to diminish NO scavenging in the blood through localized production and insignificant $\mathrm{EC} \mathrm{Ca}^{2+}$ increase in the presence of uniform eNOS distribution.

The fast reaction of $\mathrm{NO}$ with $\mathrm{Hb}$ and the resulting short half-life of $\mathrm{NO}$ make experimental measurements in presence of blood perfusion difficult. Mathematical modeling can help address the limitation and predict the NO bioavailability in the presence of erythrocyte consumption. Overproduction of $\mathrm{NO}$ in the absence of $\mathrm{Hb}$ scavengers (knockout of EC $\mathrm{Hb} \alpha$ and absence of erythrocyte consumption) have been observed in isolated arteries and co-culture models (Straub et al. 2011; Straub et al. 2012) during contractile stimuli. In the model lack of consumption in the RBC layer and zero Hb $\alpha$ concentration resulted in $\mu \mathrm{M} \mathrm{NO}$ concentration in the SMC (Figure 3.8). Regulation of NO bioavailability by EC Hba, which are preferentially located around the EC MP, through fast reactions with $\mathrm{NO}$ depending on $\mathrm{Hb} \alpha$ heme iron oxidation state have been 
suggested in the absence of NO consumption in RBC (Straub et al. 2012). The model explored this hypothesis and significant NO scavenging by Hba concentrations ( 0.01 to $0.05 \mu \mathrm{M}$ ) were observed in the absence of NO consumption in RBC layer (Figure 3.8-C). However in the presence of consumption in the RBC layer, $\mathrm{Hb} \alpha$ modulation of $\mathrm{NO}$ signaling in the model required significantly higher $\mu \mathrm{M}$ concentrations spatially localized around the MP (Figure 3.8-C). Unlike EC stimulation which results in both localized and bulk NO production, SMC stimulation results in locally produced NO from the lack of

$\mathrm{EC}$ bulk $\mathrm{Ca}^{2+}$ increase in the model. Enhanced role of $\mathrm{Hb} \alpha$ regulation was observed during SMC stimulation compared to EC stimulation through scavenging of local NO production in the absence of bulk NO production. However this predictions needs to be tested experimentally. Inhibition of contractile response after transfection of ECs with $\mathrm{Hb}$ $\alpha$ siRNA in isolated vessels have been documented (Straub et al. 2012). In the model NO scavenging by Hb $\alpha$ significant reduced the NO mediated SMC hyperpolarization feedback in the model.

\subsection{Limitation}

A number of parameter values have not been accurately quantified. In this study, we try to remain consistent with our previous $2 \mathrm{D}$ continuum EC-SMC model with respect to parameter values and whole cell currents. The 2D model is limited by the absence of quantification for the spatial distribution of important cellular components (eNOS, $\mathrm{Hb} \alpha$, $\mathrm{IP}_{3} \mathrm{Rs}$ and $\mathrm{K}_{\mathrm{Ca}}$ ). $\mathrm{Ca}^{2+}$ independent $\mathrm{NO}$ production was not considered. 


\subsection{Conclusion}

In conclusion, the developed model describes $\mathrm{Ca}^{2+}$ mediated NO signaling for regulation of vessel dilation. Model simulations were in good agreement with experiments in isolated vessels, verifying the hypotheses for the role of localized NO signaling in vascular control. The model quantifies the NO bioavailability in the SMC

during agonist stimulation of vascular cells. Model predicts localized $\mathrm{Ca}^{2+}$ signaling during the SMC stimulation around the MP and resulting NO production, and feedback response in the presence of enriched distribution of eNOS around the MP. Diminished NO feedback in the presence of EC Hba was observed in the model and was amplified from its spatial localization around the MP. Moreover, the model analyzes the relative contribution of $\mathrm{EC} \mathrm{Hb} \alpha$ and erythrocyte scavenging in the regulation of the SMC NO bioavailability and suggest that RBC perfusion will decrease the ability of $\mathrm{Hb} \alpha$ to modulate NO levels, and $\mu \mathrm{M}$ levels of EC $\mathrm{Hb} \alpha$ are necessary for a significant modulation of SMC NO availability.

\subsection{Supplement}

We performed analytical analysis using mass balance (Equation 3.9) for the NO consumption by $\mathrm{Hb} \alpha$ in EC and its consumption by the RBCs (Figure 3.9) to validate the profiles observed in the simulations.

$$
Q_{N O} V-K_{H b}[H b \alpha][N O] V-\frac{D_{N O}[N O]}{L} S_{\text {area }}=0
$$

Equation 3.10 
where $\mathrm{V}$ is the volume of the $\mathrm{EC}, \mathrm{S}_{\text {area }}$ is the surface area of the $\mathrm{EC}, \mathrm{L}$ is the distance between EC and start of RBC layer. Rearranging Equation 3.10 provides NO concentration obtained in the EC (Equation 3.11).

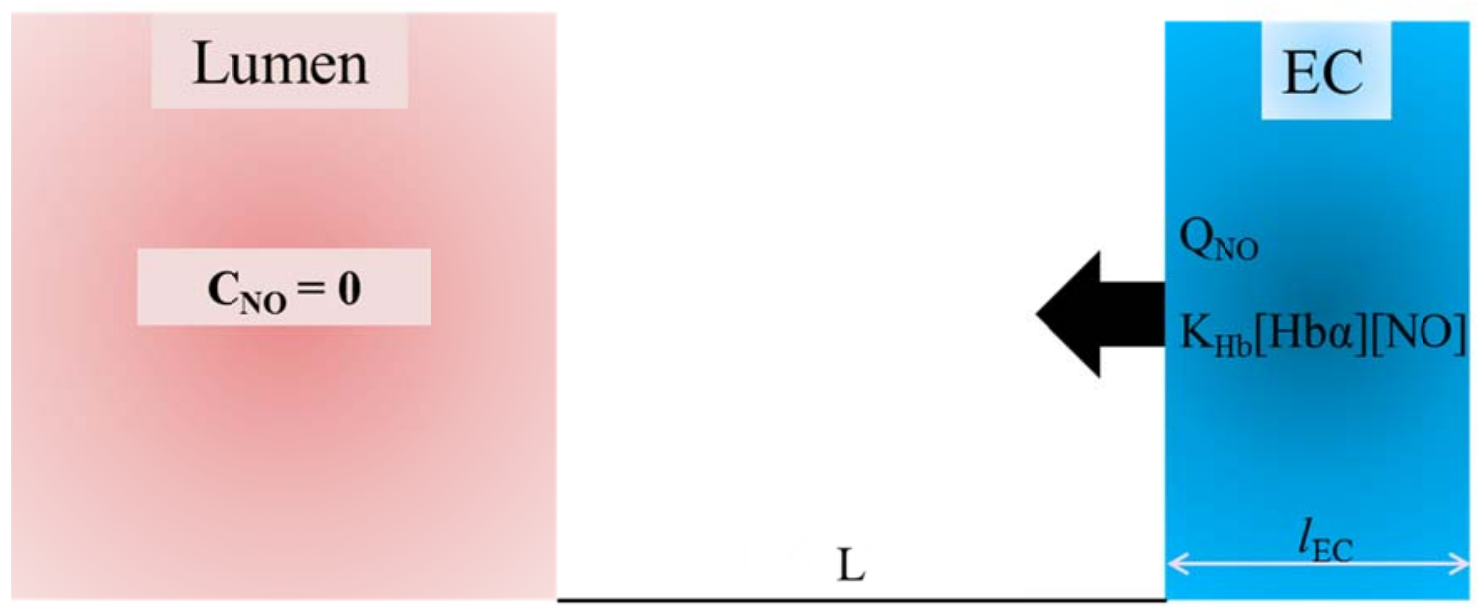

Figure 3.9 Schematic for analytical analysis of the NO consumption by $\mathrm{Hb} \alpha$ in the $\mathrm{EC}$ and its consumption by the RBCs. NO production $\left(\mathrm{Q}_{\mathrm{NO}}\right)$ is considered in the $\mathrm{EC}$ layer and its diffuses freely towards the lumen where its concentration is considered to be zero due to its rapid scavenging by the RBCs. NO scavenging in the ECs by $\mathrm{Hb} \alpha$ is considered a first order reaction $\left(\mathrm{K}_{\mathrm{Hb}}[\mathrm{Hb} \alpha][\mathrm{NO}]\right)$.

$$
[N O]=\frac{Q_{N O}}{\left(K_{H b}[H b \alpha]+\frac{D_{N O}}{L t_{E C}}\right)}
$$

Equation 3.11

where $t_{\mathrm{EC}}$ is the length of the EC.

$K_{H b}\left[H b_{\alpha}\right]$ represents the rate of NO consumption through scavenging by EC Hba and $\frac{D_{N O}}{L t_{E C}}$ represents the rate of consumption through erythrocyte layer. Using the dimension of EC considered in the model $\left(\mathrm{t}_{\mathrm{EC}}=2 \mu \mathrm{m}\right)$, distance between the RBC layer and $\mathrm{EC}(6$ 
$\mu \mathrm{m})$, NO-Hb $\alpha$ reaction rate $\mathrm{k}_{\mathrm{Hb}}$ of $100\left(\mu \mathrm{M}^{-1} \mathrm{~s}^{-1}\right)$ and $\mathrm{Hb} \alpha$ concentration of $0.5 \mu \mathrm{M}$ and

using Equation 3.11 we obtain the NO consumption rate $\left(K_{H b}\left[H b_{\alpha}\right]\right)$ of $50 \mathrm{~s}^{-1}$ by EC Hba and consumption rate in the erythrocyte layer $\left(\frac{D_{N O}}{L t_{E C}}\right)$ of $275 \mathrm{~s}^{-1}$. Hence a 5.5 fold higher consumption can be expected in the erythrocyte layer for the above parameter values.

\subsection{References}

Brasen, J. C., J. C. Jacobsen, and N. H. Holstein-Rathlou. 2012. The nanostructure of myoendothelial junctions contributes to signal rectification between endothelial and vascular smooth muscle cells. PloS one 7 (4):e33632.

Buerk, D. G., K. A. Barbee, and D. Jaron. 2011. Nitric oxide signaling in the microcirculation. Crit Rev Biomed Eng 39 (5):397-433.

Busse, R., and A. Mulsch. 1990. Calcium-dependent nitric oxide synthesis in endothelial cytosol is mediated by calmodulin. FEBS Lett 265 (1-2):133-6.

Chen, K., R. N. Pittman, and A. S. Popel. 2008. Nitric oxide in the vasculature: where does it come from and where does it go? A quantitative perspective. Antioxid Redox Signal 10 (7):1185-98.

Dora, K. A., M. P. Doyle, and B. R. Duling. 1997. Elevation of intracellular calcium in smooth muscle causes endothelial cell generation of $\mathrm{NO}$ in arterioles. Proc Natl Acad Sci U S A 94 (12):6529-34.

Eich, R. F., T. Li, D. D. Lemon, D. H. Doherty, S. R. Curry, J. F. Aitken, A. J. Mathews, K. A. Johnson, R. D. Smith, G. N. Phillips, Jr., and J. S. Olson. 1996. Mechanism of NO-induced oxidation of myoglobin and hemoglobin. Biochemistry 35 (22):6976-83.

Furchgott, R. F., and J. V. Zawadzki. 1980. The obligatory role of endothelial cells in the relaxation of arterial smooth muscle by acetylcholine. Nature 288 (5789):373-6.

Gladwin, M. T., and D. B. Kim-Shapiro. 2012. Vascular biology: Nitric oxide caught in traffic. Nature 491 (7424):344-5.

Heberlein, K. R., A. C. Straub, and B. E. Isakson. 2009. The myoendothelial junction: breaking through the matrix? Microcirculation 16 (4):307-22. 
Herold, S., M. Exner, and T. Nauser. 2001. Kinetic and mechanistic studies of the NO*mediated oxidation of oxymyoglobin and oxyhemoglobin. Biochemistry 40 (11):3385-95.

Ignarro, L. J., G. M. Buga, K. S. Wood, R. E. Byrns, and G. Chaudhuri. 1987. Endothelium-derived relaxing factor produced and released from artery and vein is nitric oxide. Proc Natl Acad Sci U S A 84 (24):9265-9.

Isakson, B. E. 2008. Localized expression of an $\operatorname{Ins}(1,4,5) \mathrm{P} 3$ receptor at the myoendothelial junction selectively regulates heterocellular $\mathrm{Ca} 2+$ communication. J Cell Sci 121 (Pt 21):3664-73.

Isakson, B. E., S. I. Ramos, and B. R. Duling. 2007. Ca2+ and inositol 1,4,5trisphosphate-mediated signaling across the myoendothelial junction. Circ Res 100 (2):246-54.

Iwakiri, Y., A. Satoh, S. Chatterjee, D. K. Toomre, C. M. Chalouni, D. Fulton, R. J. Groszmann, V. H. Shah, and W. C. Sessa. 2006. Nitric oxide synthase generates nitric oxide locally to regulate compartmentalized protein S-nitrosylation and protein trafficking. Proc Natl Acad Sci US A 103 (52):19777-82.

Kansui, Y., C. J. Garland, and K. A. Dora. 2008. Enhanced spontaneous Ca2+ events in endothelial cells reflect signalling through myoendothelial gap junctions in pressurized mesenteric arteries. Cell Calcium 44 (2):135-46.

Kapela, A., A. Bezerianos, and N. M. Tsoukias. 2008. A mathematical model of Ca2+ dynamics in rat mesenteric smooth muscle cell: agonist and NO stimulation. $J$ Theor Biol 253 (2):238-60.

Kapela, A., A. Bezerianos, and N. M. Tsoukias. 2009. A mathematical model of vasoreactivity in rat mesenteric arterioles: I. Myoendothelial communication. Microcirculation 16 (8):694-713.

Kapela, A., S. Nagaraja, and N. M. Tsoukias. 2010. A mathematical model of vasoreactivity in rat mesenteric arterioles. II. Conducted vasoreactivity. Am J Physiol Heart Circ Physiol 298 (1):H52-65.

Kapela, A., and N. M. Tsoukias. 2011. Multiscale FEM modeling of vascular tone: from membrane currents to vessel mechanics. IEEE Trans Biomed Eng 58 (12):3456-9.

Kerr, P. M., R. Tam, K. Ondrusova, R. Mittal, D. Narang, C. H. Tran, D. G. Welsh, and F. Plane. 2012. Endothelial feedback and the myoendothelial projection. Microcirculation 19 (5):416-22.

Ledoux, J., M. S. Taylor, A. D. Bonev, R. M. Hannah, V. Solodushko, B. Shui, Y. Tallini, M. I. Kotlikoff, and M. T. Nelson. 2008. Functional architecture of 
inositol 1,4,5-trisphosphate signaling in restricted spaces of myoendothelial projections. Proc Natl Acad Sci US A 105 (28):9627-32.

Lewis, R. S., and W. M. Deen. 1994. Kinetics of the reaction of nitric oxide with oxygen in aqueous solutions. Chem Res Toxicol 7 (4):568-74.

Nagaraja, S., A. Kapela, C. H. Tran, D. G. Welsh, and N. M. Tsoukias. 2013. Role of microprojections in myoendothelial feedback--a theoretical study. J Physiol 591 (Pt 11):2795-812.

Nagaraja, S., A. Kapela, and N. M. Tsoukias. 2012. Intercellular communication in the vascular wall: a modeling perspective. Microcirculation 19 (5):391-402.

Palmer, R. M., A. G. Ferrige, and S. Moncada. 1987. Nitric oxide release accounts for the biological activity of endothelium-derived relaxing factor. Nature 327 (6122):524-6.

Qian, J., Q. Zhang, J. E. Church, D. W. Stepp, R. D. Rudic, and D. J. Fulton. 2010. Role of local production of endothelium-derived nitric oxide on cGMP signaling and Snitrosylation. Am J Physiol Heart Circ Physiol 298 (1):H112-8.

Rahaman, M. M., and A. C. Straub. 2013. The emerging roles of somatic globins in cardiovascular redox biology and beyond. Redox Biol 1 (1):405-10.

Sandow, S. L., R. E. Haddock, C. E. Hill, P. S. Chadha, P. M. Kerr, D. G. Welsh, and F. Plane. 2009. What's where and why at a vascular myoendothelial microdomain signalling complex. Clin Exp Pharmacol Physiol 36 (1):67-76.

Silva, H. S., A. Kapela, and N. M. Tsoukias. 2007. A mathematical model of plasma membrane electrophysiology and calcium dynamics in vascular endothelial cells. Am J Physiol Cell Physiol 293 (1):C277-93.

Straub, A. C., M. Billaud, S. R. Johnstone, A. K. Best, S. Yemen, S. T. Dwyer, R. LooftWilson, J. J. Lysiak, B. Gaston, L. Palmer, and B. E. Isakson. 2011. Compartmentalized connexin 43 s-nitrosylation/denitrosylation regulates heterocellular communication in the vessel wall. Arterioscler Thromb Vasc Biol 31 (2):399-407.

Straub, A. C., A. W. Lohman, M. Billaud, S. R. Johnstone, S. T. Dwyer, M. Y. Lee, P. S. Bortz, A. K. Best, L. Columbus, B. Gaston, and B. E. Isakson. 2012. Endothelial cell expression of haemoglobin alpha regulates nitric oxide signalling. Nature 491 (7424):473-7.

Tran, C. H., M. S. Taylor, F. Plane, S. Nagaraja, N. M. Tsoukias, V. Solodushko, E. J. Vigmond, T. Furstenhaupt, M. Brigdan, and D. G. Welsh. 2012. Endothelial Ca2+ wavelets and the induction of myoendothelial feedback. Am J Physiol Cell Physiol 302 (8):C1226-42. 
Tsoukias, N. M. 2008. Nitric oxide bioavailability in the microcirculation: insights from mathematical models. Microcirculation 15 (8):813-34.

Tsoukias, N. M., M. Kavdia, and A. S. Popel. 2004. A theoretical model of nitric oxide transport in arterioles: frequency- vs. amplitude-dependent control of cGMP formation. Am J Physiol Heart Circ Physiol 286 (3):H1043-56.

Tsoukias, N. M., and A. S. Popel. 2002. Erythrocyte consumption of nitric oxide in presence and absence of plasma-based hemoglobin. Am J Physiol Heart Circ Physiol 282 (6):H2265-77.

Yashiro, Y., and B. R. Duling. 2000. Integrated $\mathrm{Ca}(2+)$ signaling between smooth muscle and endothelium of resistance vessels. Circ Res 87 (11):1048-54. 
CHAPTER 4-INTRACELLULAR CALCIUM WAVES IN VASCULAR CELLS

This chapter is to be submitted to Biophysical Journal (with modifications) as Parikh, J., A. Kapela and N. M.Tsoukias. "Oscillatory calcium waves in vascular cells: wave velocity dependence on membrane electrophysiology" 


\subsection{Abstract}

Agonist stimulation of both smooth muscle (SMC) and endothelial (EC) cells results in calcium $\left(\mathrm{Ca}^{2+}\right)$ mobilization that is associated with diverse cellular functions. Spatiotemporal $\mathrm{Ca}^{2+}$ events in the form of intracellular $\mathrm{Ca}^{2+}$ waves have been reported in SMCs and ECs of different vascular beds. The underlying mechanisms and physiological roles are under investigation. Here we use detailed mathematical models of $\mathrm{Ca}^{2+}$ dynamics and electrophysiology to investigate the mechanisms that initiate and modulate $\mathrm{Ca}^{2+}$ waves in these two cell types. The models reproduce wave like activity when a gradient in store (i.e. inositol triphosphate, ryanodine) or membrane (i.e. adrenergic, muscarinic) receptor density is assumed. The resulting intracellular waves depend on the intrinsic ability of the cells for store-dependent oscillations. These repetitive/oscillatory waves require a weak propagating signal to synchronize neighboring domains, balancing uneven receptor distribution and/or store dynamics. Small $\mathrm{Ca}^{2+}$ fluxes in the axial direction are adequate to maintain wave activity. Model predicts that the membrane potential $\left(V_{\mathrm{m}}\right)$ can affect wave velocity through modulation of transmembrane $\mathrm{Ca}^{2+}$ influx (i.e. via voltage sensitive $\mathrm{Ca}^{2+}$ channels in SMCs or by altering the electrochemical gradient for $\mathrm{Ca}^{2+}$ influx in ECs). Depolarizing currents in SMCs (i.e. via non-selective cation channels and $\mathrm{Ca}^{2+}$ activated chloride channels) and hyperpolarizing currents in ECs (i.e. small and intermediate conductance $\mathrm{Ca}^{2+}$ activated $\mathrm{K}^{+}$channels) promote an increase in wave velocity and transition to whole-cell oscillations. The model predicts that the frequency and velocity of these oscillatory $\mathrm{Ca}^{2+}$ waves may depend on agonist stimulus strength and thus waves may encode information of physiological importance. 


\subsection{Introduction}

Diverse cellular events like contraction, relaxation, gene expression, cell death and proliferation are associated with cytoplasmic calcium $\left(\mathrm{Ca}^{2+}\right)$ mobilization (Foskett et al. 2007). Transmembrane $\mathrm{Ca}^{2+}$ fluxes, store uptake and release generate local or global $\mathrm{Ca}^{2+}$ events. In the vasculature, spatio-temporal $\mathrm{Ca}^{2+}$ events ranging from localized $\mathrm{Ca}^{2+}$ activity like sparks, puffs, sparklets or pulsars to well organized signaling events like waves or whole cell oscillations have been reported (Amberg and Navedo 2013). Moreover $\mathrm{Ca}^{2+}$ events may also propagate to adjacent cells forming intercellular $\mathrm{Ca}^{2+}$ waves (Halidi et al. 2011; Seppey et al. 2010; Socha et al. 2012). Intracellular $\mathrm{Ca}^{2+}$ waves have been observed in vascular cells with different propagation velocities (Jaffe 2010; Narayanan, Adebiyi, and Jaggar 2012). The physiological relevance of $\mathrm{Ca}^{2+}$ wave activity is still unclear, although, a role in cell proliferation and gene expression (Narayanan, Adebiyi, and Jaggar 2012) or in the regulation of vascular tone(Wang et al. 2010; Amberg and Navedo 2013) has been suggested.

The reported intracellular $\mathrm{Ca}^{2+}$ waves be attributed two different mechanisms based on the experimental technique undertaken to generate them; Stochastic $\mathrm{Ca}^{2+}$ waves and repetitive/oscillatory $\mathrm{Ca}^{2+}$ waves. The first type is often a result of localized $\mathrm{Ca}^{2+}$ release due the stochastic/random opening of store receptors (McCarron et al. 2010; Marchant, Callamaras, and Parker 1999; Halidi et al. 2011). If the localized release is sufficiently high, $\mathrm{Ca}^{2+}$ diffusion away from this point may cause calcium induced calcium release (CICR) resulting in single or frequently appearing $\mathrm{Ca}^{2+}$ wave traversing across the cell. The point of origin of these waves can be random depending on the site of localized increase of $\mathrm{Ca}^{2+}$. In experiments localized increase in $\mathrm{Ca}^{2+}$ through photolysis of caged 
$\mathrm{IP}_{3}$ has often been utilized to examine the underlying mechanism of $\mathrm{Ca}^{2+}$ wave propagation (McCarron et al. 2010) and several mathematical models examining the generation of $\mathrm{Ca}^{2+}$ puffs and their conversion to waves have been presented (Falcke 2003; Falcke, Or-Guil, and Bar 2000; Falcke, Tsimring, and Levine 2000). On the contrary the second type of $\mathrm{Ca}^{2+}$ wave i.e. repetitive/oscillatory $\mathrm{Ca}^{2+}$ waves often appear as a result of constant agonist stimulation of cultured vascular cells/intact vessel to produce a global $\mathrm{Ca}^{2+} / \mathrm{IP}_{3}$ response. Such $\mathrm{Ca}^{2+}$ waves usually have a fixed initiation site and frequency of appearance.

Agonist induced oscillatory $\mathrm{Ca}^{2+}$ waves have been observed in SMCs (Dai et al. 2007; Dai et al. 2006; Lee et al. 2001; McCarron et al. 2010; Peng et al. 2001; Ruehlmann et al. 2000; Syyong et al. 2009) and ECs (Huser and Blatter 1997; Isshiki et al. 1998; Socha et al. 2012; Beliveau, Lapointe, and Guillemette 2011) of different species. Agonist stimulus results in elevated $\mathrm{Ca}^{2+}$ and $\mathrm{IP}_{3}$ levels and may induce stable localized or whole cell $\mathrm{Ca}^{2+}$ oscillations in the cells by bringing the $\mathrm{Ca}^{2+}$ levels within the oscillatory domain (Kapela, Bezerianos, and Tsoukias 2008). $\mathrm{Ca}^{2+}$ oscillations depend on calcium induced calcium release (CICR) through either of $\mathrm{IP}_{3}$ receptor $\left(\mathrm{IP}_{3} \mathrm{R}\right)$ channel (Bai, Edelmann, and Sanderson 2009; Lamont and Wier 2004; McCarron et al. 2010) and/or Ryanodine receptor (RyR) channels (Lagaud et al. 1999; Dai et al. 2006; Balemba et al. 2006). The spatial heterogeneity essential for generation of $\mathrm{Ca}^{2+}$ waves may result for non-uniform distribution of the cellular components. Gradient distribution of both $\mathrm{IP}_{3}$ and RyRs i.e. higher density of these receptors on one side of cell compared to other was shown to be essential for generation and propagation of $\mathrm{Ca}^{2+}$ waves in airways SMC model (Wang et al. 2010). Both $\mathrm{Ca}^{2+}$ and $\mathrm{IP}_{3}$ mobilization was required for $\mathrm{Ca}^{2+}$ wave generation and 
propagation in (McCarron et al. 2010). $V_{\mathrm{m}}$ can modulate $\mathrm{Ca}^{2+}$ levels, directly through activation/inactivation of voltage dependent $\mathrm{Ca}^{2+}$ channels or indirectly by affecting the electrochemical gradient for $\mathrm{Ca}^{2+}$ entry via non-selective cation channels (NSC).

Wide variation in $\mathrm{Ca}^{2+}$ wave velocities have been reported (Jaffe 2010; Narayanan, Adebiyi, and Jaggar 2012). While several experiments and models have been undertaken to understand the origin and mechanism of oscillatory $\mathrm{Ca}^{2+}$ waves the mechanism(s) that modulates wave velocities has not been elucidated. Different cellular components have been suggested to modulate this oscillatory $\mathrm{Ca}^{2+}$ waves and wide variation in velocity (1$126 \mu \mathrm{m} / \mathrm{sec}$ ) and propagation distances (8.8-50 $\mu \mathrm{m}$ ) have also been reported (Jaffe 2010; Narayanan, Adebiyi, and Jaggar 2012). The physiological significance of accelerating/decelerating wave velocity is not yet understood but theoretical consideration suggest that high velocities and transition to whole cell oscillations may increase contractility of vascular SMCs (Wang et al. 2010). Transition from oscillatory calcium waves to whole cell oscillations have also been observed experimentally (Peng et al. 2001) and mathematical models explaining these transitions have also been reported (Jacobsen et al. 2007; Peng et al. 2001; Postnov et al. 2011). Calcium activated chloride channels $\left(\mathrm{Cl}_{\mathrm{Ca}}\right)$ have been shown to be the main determinant for this conversion to whole cells waves.

Several minimal deterministic reaction-diffusion models of $\mathrm{Ca}^{2+}$ waves, describing $\mathrm{Ca}^{2+}$ and $\mathrm{IP}_{3}$ dynamics have been undertaken. They differ in the underlying assumption of the oscillatory mechanism. (For example $\mathrm{Ca}^{2+}$ waves arising from $\mathrm{IP}_{3} \mathrm{R}$ are consider in (Atri et al. 1993; Dupont and Goldbeter 1994; Sneyd et al. 1995), oscillation through combination of $I_{3} R$ and RyR in (Wang et al. 2010). Moreover, the models differ in type 
of stimulus i.e. Ca2+ waves generated from localized $\mathrm{Ca}^{2+}$ increase (Atri et al. 1993; Dupont and Goldbeter 1994) which propagates across the cell or under uniform stimulus throughout the cell (Wang et al. 2010) (e.x. through rise of $\mathrm{IP}_{3}$ levels throughout the cell). Very few models have examined the effect of Vm on generation and modulation of the oscillatory $\mathrm{Ca}^{2+}$ waves. Jacobsen et al.(Jacobsen et al. 2007) incorporates $V_{\mathrm{m}}$ dynamics and transmembrane currents in their cytosolic oscillator model to examine the effect of $\mathrm{Ca}^{2+}$ activated chloride channels $\left(\mathrm{Cl}_{\mathrm{Ca}}\right)$ in tranisition from waves to whole cell oscillatoions, however they lack the presence of NSC channels, RyRs, $\mathrm{Ca}^{2+}$-dependent PLC activity, intracellular $\mathrm{IP}_{3}$ diffusion, or activation of $\mathrm{BK}_{\mathrm{Ca}}$ channels by cGMP. Postnov et al. (Postnov et al. 2011) went with a minimal model to understand the transition to whole cell oscillations. The proposed mathematical models (Jacobsen et al. 2007; Postnov et al. 2011; Wang et al. 2010) provides valuable insights into generation and propagation of the intracellular $\mathrm{Ca}^{2+}$ waves and also transition to whole cell oscillations. These earlier models can be further advanced by incorporation of potentially important components and pathways and improve quantitative descriptions.

In this theoretical study, we develop detailed mathematical models of integrated $\mathrm{Ca}^{2+}$ and $V_{\mathrm{m}}$ dynamics to try and understand the mechanism generating and mediating these oscillatory $\mathrm{Ca}^{2+}$ waves and attempt to unravel the underlying pathways modulating the velocities and frequency of the agonist induced oscillatory intracellular $\mathrm{Ca}^{2+}$ wave in SMCs and ECs. Simulations suggest that complex pathways exist in the generation and propagation of oscillatory $\mathrm{Ca}^{2+}$ wave and different cellular components may play important roles in the two cell types. Moreover the model reveals that several pathways may simultaneously regulate the velocity and frequency of the oscillatory $\mathrm{Ca}^{2+}$ waves and 
the relative importance of the pathways depend on the simulation type and intensity. The model also assists in understanding the effect of non-homogenous spatial distribution of cellular components in both the cell types.

\subsection{Methods}

We have previously developed detailed models of integrated $\mathrm{Ca}^{2+}$ and $V_{\mathrm{m}}$ dynamics in isolated EC and SMC (Kapela, Bezerianos, and Tsoukias 2008; Silva, Kapela, and Tsoukias 2007), based primarily on data from rat mesenteric arteries (RMA). The single cells models were extended to incorporate spatial heterogeneity of cellular components and intracellular gradients for $\mathrm{Ca}^{2+}, \mathrm{IP}_{3}$ and other ions $\left(\mathrm{Cl}^{-}, \mathrm{Na}^{+}\right.$and $\left.\mathrm{K}^{+}\right)$. We developed a FEM model to account for the spatial gradients of cellular components inside the EC and SMC essential for $\mathrm{Ca}^{2+}$ wave generation. While a 3D cylindrical axisymmetric model of EC and SMC was implemented in (Kapela and Tsoukias 2011), here we assumed a simplified geometry with EC and SMC being represented as rectangular structures 100 $\mu \mathrm{m}$ long and $6 \mu \mathrm{m}$ wide. The model implements only half of the EC and SMC. The results in the remaining half are assumed to be symmetrical. Electro-diffusion for the ionic species was taken into account. This allows implementation of voltage and concentration dependent membrane currents as boundary equations. The resulting differential equations describing the intracellular gradients for each ionic species $\mathrm{A}\left(\mathrm{Ca}^{2+}\right.$, $\mathrm{Cl}^{-}, \mathrm{Na}^{+}$and $\mathrm{K}^{+}$) and second messenger $\mathrm{IP}_{3}$ (Kapela, Bezerianos, and Tsoukias 2008) in the two cells SMC/EC (Equation 4.1- 4.4): The transport of $\mathrm{Ca}^{2+}$ across the cell is given by Equation 4.1 


$$
\delta_{\text {buff }, C a} \frac{\partial[C a]_{i}}{\partial t}=\nabla \cdot\left(D_{C a} \nabla[C a]_{i}+z_{C a} F \frac{D_{C a}}{R T}[C a]_{i} \nabla V_{i}\right)+R_{r e l}-R_{\text {uptake }}
$$

\section{Equation 4.1}

where $\delta_{b u f f, C a}$ accounts for rapid buffering in cytosol and store, $R_{r e l}$ defines the release of $\mathrm{Ca}^{2+}$ from store via $\mathrm{IP}_{3} \mathrm{R}$ and $\mathrm{RyR}$ receptors and a leak current through the stores, $R_{\text {uptake }}$ defines the re-sequestration of $\mathrm{Ca}^{2+}$ by the store through uptake by SERCA. The expressions for buffering, release and uptake are same as in our previous models (Kapela, Bezerianos, and Tsoukias 2008; Silva, Kapela, and Tsoukias 2007). $D_{C a}$ is the diffusion coefficient of $\mathrm{Ca}^{2+}$ (TABLE 1). $z_{C a}$ is the valence of $\mathrm{Ca}^{2+}$ ion. $F$ is the Faradays constant. $V_{i}$ denotes intracellular electrical potential. The molar fluxes on the membrane for $\mathrm{Ca}^{2+}$ are calculated using the sum of all $\mathrm{Ca}^{2+}$ membrane currents $\left(\sum_{K} I_{C a, K}\right)$ and implemented as a boundary condition (Equation 4.2)

$$
-n \cdot N_{C a}=\frac{1}{Z_{C a} F} \sum_{K} I_{C a, K}
$$

Equation 4.2

where $\mathrm{n}$ is the normal to the surface, $N_{C a}$ is the flux of $\mathrm{Ca}^{2+}$ across the membrane, $K$ refers to all membrane currents for each ionic species.

The transport of $\mathrm{IP}_{3}$ is assumed to be independent of the membrane potential dynamics and implemented using simple diffusion equation (Equation 4.3) 


$$
\frac{\partial\left[I P_{3}\right]_{i}}{\partial t}=\nabla \cdot\left(D_{I P_{3}} \nabla\left[I P_{3}\right]_{i}\right)-R_{\operatorname{deg} \text { radation }}
$$

Equation 4.3

where $D_{I P_{3}}$ is the diffusion coefficient of $\mathrm{Ca}^{2+}$ (TABLE 1). $R_{\text {degradation }}$ is sink term which includes expression for cytosolic degradation of $\mathrm{IP}_{3}$ (Kapela, Bezerianos, and Tsoukias 2008; Silva, Kapela, and Tsoukias 2007).

The molar flux for $\mathrm{IP}_{3}$ production $\left(J_{P L C, I P_{3}}\right)$ is fulfilled as a boundary condition (Equation 4.4).

$$
-n \cdot N_{I P_{3}}=J_{P L C, I P_{3}}
$$

Equation 4.4

Transport of rest of the ionic species $[S]_{i}$ where $S=\mathrm{Na}^{+}, \mathrm{K}^{+}, \mathrm{Cl}^{-}$is described by Equation 4.5.

$$
\frac{\partial[S]_{i}}{\partial t}=\nabla \cdot\left(D_{S} \nabla[S]_{i}+z_{s} F \frac{D_{s}}{R T}[S]_{i} \nabla V_{i}\right)
$$

Equation 4.5

$D_{S}$ is the diffusion coefficient of ionic species $S, z_{S}$ is the charge number of ionic species $S$.

The molar fluxes on the membrane for ionic species $\mathrm{S}$ are calculated using the sum of all membrane currents $\left(\sum_{K} I_{S, K}\right)$ for each ionic species $\mathrm{S}$ and implemented as a boundary condition (Equation. 6) 


$$
-n \cdot N_{S}=\frac{1}{Z_{S} F} \sum_{K} I_{S, K}
$$

Equation 4.6

The change in membrane potential across the length of cell is given by (Equation 4.7) and is dependent on sum of all membrane currents $(\mathrm{K})$ for each ionic species $\mathrm{A}\left(\sum_{K} I_{A, K}\right)$

$$
-n . F \sum Z_{A}\left(D_{A} \nabla[A]_{i}+Z_{A} F u \frac{D_{A}}{R T}[A]_{i} \nabla V_{i}\right)=\sum_{K} I_{A, K}
$$

\section{Equation 4.7}

where $\mathrm{A}=\mathrm{Ca}^{2+}, \mathrm{Na}^{+}, \mathrm{K}^{+}$and $\mathrm{Cl}^{-}, \mathrm{D}_{\mathrm{A}}$ is the diffusivity of the individual ions (Table 4.1) FEM models for both the cells were developed using the chemical engineering module of the COMSOL multiphysics software. The details of the FEM models are given in supplement.

In order to reduce the computational overheads of a 2-D FEM model and to better understand/estimate the role diffusional fluxes of ions and second messenger, we reduced the 2-D model by neglecting the concentration gradients across the width of the cell and assuming only the axial fluxes, to result in 1-D axial geometry for examining oscillatory $\mathrm{Ca}^{2+}$ waves. Reducing to $1-\mathrm{D}$ resulted in the boundary conditions (membrane fluxes) of the 2-D model to be incorporated into the equation of transport of each species itself. The resulting equations are described in Equation 4.8-4.11 (supplement (section 4.9)).

Intracellular transport for $\mathrm{Ca}^{2+}$ in 1-D is described using Equation 4.8

$$
\delta_{\text {buf }, C a} \frac{\partial[C a]_{i}}{\partial t}=D_{C a} \frac{\partial^{2}[C a]_{i}}{\partial x^{2}}+\frac{\partial}{\partial x}\left(\frac{D_{C a} z_{C a} F[C a]_{i}}{R T} \frac{\partial[V]_{i}}{\partial x}\right)+\frac{I_{C a, \text { rel }}(x)-I_{C a, \text { uptake }}(x)+\sum_{K} I_{C a, K}(x)}{Z_{C a} F}
$$

Equation 4.8 
where $I_{\text {Ca,rel }}(x), I_{\text {Ca,uptake }}(x)$ are the current densities $\left(\frac{\mathrm{pA}}{\mu \mathrm{m}^{3}}\right)$ for $\mathrm{Ca}^{2+}$ release and uptake from the store respectively at any axial point $\mathrm{x}$ across the length of the cell. $\sum_{K} I_{C a, K}(x)$ is the summation of membrane current density $\left(\frac{\mathrm{pA}}{\mu \mathrm{m}^{3}}\right)$ of all the membrane $\mathrm{Ca}^{2+}$ channel currents at any point $\mathrm{x}$ across the length of the cell.

Intracellular transport for $\mathrm{IP}_{3}$ in 1-D is given by Equation 4.9

$$
\frac{\partial\left[I P_{3}\right]_{i}}{\partial t}=D_{I P_{3}} \frac{\partial^{2}\left[I P_{3}\right]_{i}}{\partial x^{2}}-R_{\operatorname{deg} r a d a t i o n}(x)-R_{P L C, I_{3}}(x)
$$

Equation 4.9

where $R_{P L C, I P_{3}}=\frac{r_{h, g}}{\gamma_{G}}\left[P I P_{2}\right], R_{\text {degradation }}=K_{\operatorname{deg} G G}\left[I P_{3}\right]_{i}$ (Kapela, Bezerianos, and Tsoukias 2008)

The transport in 1-D for rest of the ionic species $\mathrm{S}$ is reduced to Equation 4.10

$$
\frac{\partial[S]_{i}}{\partial t}=D_{S} \frac{\partial^{2}[S]_{i}}{\partial x^{2}}+\frac{\partial}{\partial x}\left(\frac{D_{S} Z_{S} F[C a]_{i}}{R T} \frac{\partial[V]_{i}}{\partial x}\right)+\frac{\sum_{K} I_{S, K}(x)}{Z_{S} F}
$$

Equation 4.10

where $\sum_{K} I_{S, K}(x)$ is the summation of membrane current desnities for each ionic species $S\left(\frac{\mathrm{pA}}{\mu \mathrm{m}^{3}}\right)$

Finally the change in membrane potential with respect to time across the length of cell in 1-D is given by Equation 4.11 


$$
C_{m} \frac{\partial V_{i}}{\partial t}=\frac{\partial}{\partial x}\left(F \sum Z_{A}\left(-D_{A} \frac{\partial[A]_{i}}{\partial x}-\frac{Z_{A} F u_{m s}[A]_{i}}{R T} \frac{\partial V_{i}}{\partial x}\right)\right)-\sum_{K} I_{A, K}(x)
$$

Equation 4.11

here $\sum_{K} I_{A, K}(x)$ is the summation of total membrane current density for all ions including second messenger, $\mathrm{A}\left(\frac{\mathrm{pA}}{\mu \mathrm{m}^{3}}\right)$. Discretization of the single cell models of EC and SMC

(Figure 4.1-A) to form a multi-compartmental model as discussed in supplement (section 4.9) was performed to solve for oscillatory $\mathrm{Ca}^{2+}$ wave model. The cellular components and pathways examined for the individual compartments in SMC and EC has been shown in Figure 4.1-B and C.

A two pool model have been suggested for generating histamine induced $\mathrm{Ca}^{2+}$ oscillations in human endothelial cells (Paltauf-Doburzynska et al. 2000). In their study RyR are shown to cause the calcium induced calcium release following opening or $\mathrm{IP}_{3} \mathrm{Rs}$. However recent studies suggest the absence of RyRs in particular EC types but have shown the presence of more than one of subtypes of $\mathrm{IP}_{3} \mathrm{Rs}$ (Ledoux et al. 2008) $\left(\mathrm{IP}_{3} \mathrm{R}-1\right.$, $\mathrm{IP}_{3} \mathrm{R}-2$, and $\left.\mathrm{IP}_{3} \mathrm{R}-3\right)$. Varying proportions of these subtypes depending on cell type and species are observed. To the best of our knowledge no detailed models for $\mathrm{Ca}^{2+}$ oscillation in vascular EC has yet been reported. We formulated a two pool model(Paltauf-Doburzynska et al. 2000; Parthimos, Edwards, and Griffith 1999) to account for more than one these subtypes of $\mathrm{IP}_{3}$ receptor in $\mathrm{EC}$ as shown in Figure 4.1-C and resulted in generate the $\mathrm{Ca}^{2+}$ oscillation which were absent in the original single cell model (Silva, Kapela, and Tsoukias 2007). The first pool maintains the steady state $\mathrm{Ca}^{2+}$ levels with the second pool inducing the oscillations on the top of this steady state level. 
The current formulations (Equation 4.12-4.14) and parameter values describing the second pool in the model (Table 4.1) were chosen from Parthimos et al.(Parthimos,

Edwards, and Griffith 1999) .

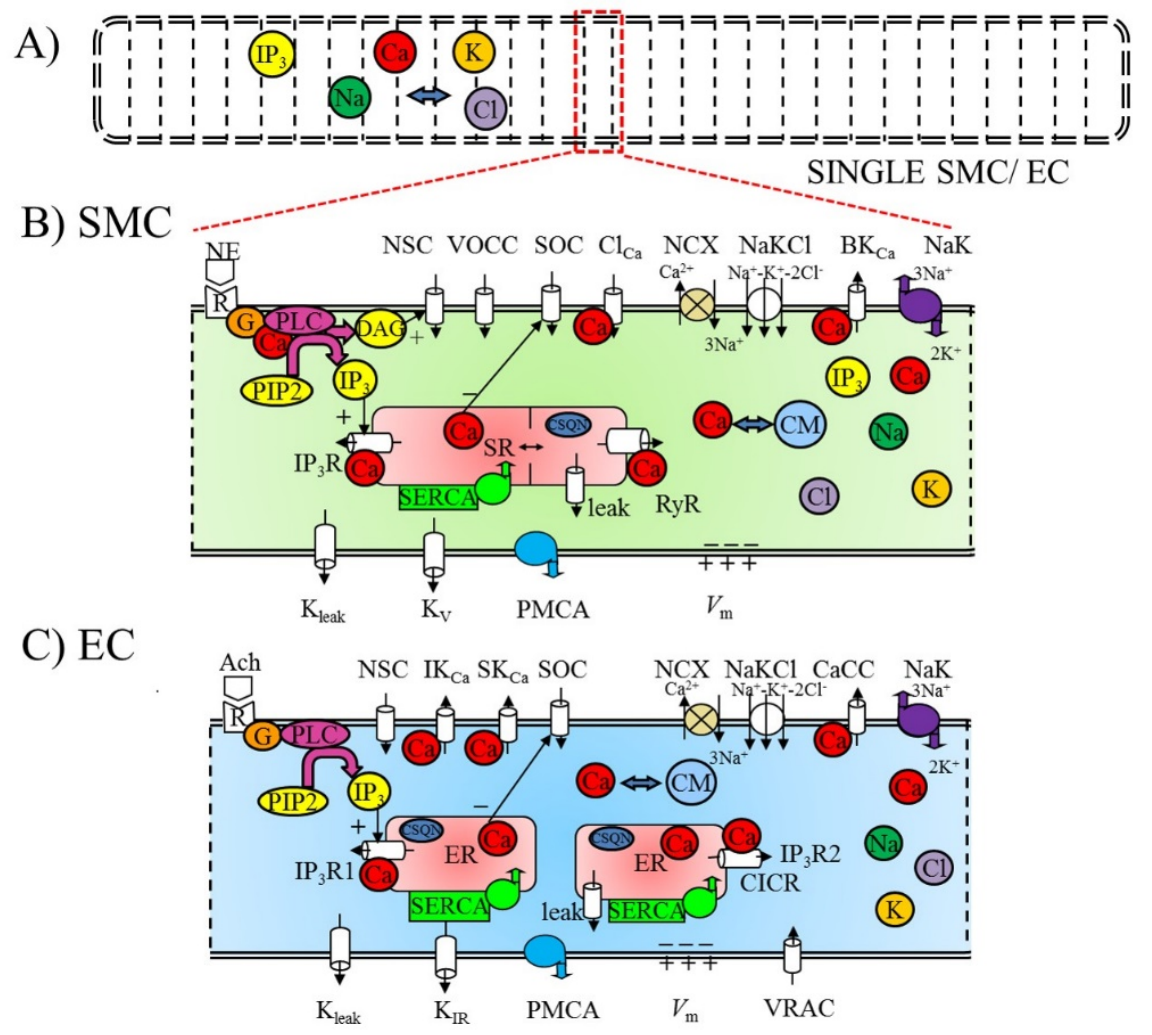

Figure 4.1 (A) Schematic diagram of single cell EC and SMC model divided into compartments (B) Zoomed in view of a single SMC compartment showing all the incorporated components. $\mathrm{BK}_{\mathrm{Ca}}$-largeconductance $\mathrm{Ca}^{2+}$-activated $\mathrm{K}^{+}$channels; SOC - store-operated channel; NSC - nonselective cation channel, $\mathrm{CaCC}$ and $\mathrm{Cl}_{\mathrm{Ca}}-\mathrm{Ca}^{2+}$-activated chloride channel; $\mathrm{NaK}-\mathrm{Na}^{+}-\mathrm{K}^{+}$-ATPase; PMCA - plasma membrane $\mathrm{Ca}^{2+}$-ATPase; $\mathrm{NCX}-\mathrm{Na}^{+} / \mathrm{Ca}^{2+}$ exchanger; $\mathrm{NaKCl}-\mathrm{Na}^{+}-\mathrm{K}^{+}-\mathrm{Cl}^{-}$cotransport; $\mathrm{K}_{\mathrm{v}}-$ voltagedependent $\mathrm{K}^{+}$channel; $\mathrm{K}_{\text {leak }}$ - unspecified $\mathrm{K}^{+}$leak current; VOCC - voltage-operated $\mathrm{Ca}^{2+}$ channels; $\mathrm{SR}$ sarcoplasmic reticulum; $\mathrm{IP}_{3} \mathrm{R}-\mathrm{IP}_{3}$ receptor; $\mathrm{RyR}$ - ryanodine receptor; SERCA - $\mathrm{SR} \mathrm{Ca}^{2+}$-ATPase; CSQN - calsequestrin; CM - calmodulin; R - receptor; G - G-protein; DAG - diacylglycerol; PLC phospholipase C; sGC - soluble guanylate cyclase; cGMP - cyclic guanosine monophosphate. (C) Zoomed in view of a single $\mathrm{EC}$ compartment revealing all the incorporated components. $\mathrm{K}_{\mathrm{ir}}$ - inward rectifier $\mathrm{K}^{+}$ channel; VRAC - volume-regulated anion channel; $\mathrm{SK}_{\mathrm{Ca}}, \mathrm{IK}_{\mathrm{Ca}}$ and $\mathrm{BK}_{\mathrm{Ca}}$ - small-, intermediate-, and largeconductance $\mathrm{Ca}^{2+}$-activated $\mathrm{K}^{+}$channels; SOC - store-operated channel; NSC - nonselective cation channel, $\mathrm{CaCC}$ and $\mathrm{Cl}_{\mathrm{Ca}}-\mathrm{Ca}^{2+}$-activated chloride channel; $\mathrm{NaK}-\mathrm{Na}^{+}-\mathrm{K}^{+}$-ATPase; PMCA - plasma membrane $\mathrm{Ca}^{2+}$-ATPase; $\mathrm{NCX}-\mathrm{Na}^{+} / \mathrm{Ca}^{2+}$ exchanger; $\mathrm{NaKCl}-\mathrm{Na}^{+}-\mathrm{K}^{+}-\mathrm{Cl}^{-}$cotransport; $\mathrm{K}_{\mathrm{v}}-$ voltagedependent $\mathrm{K}^{+}$channel; ; $\mathrm{K}_{\text {leak }}$ - unspecified $\mathrm{K}^{+}$leak current; $\mathrm{ER}$ - endoplasmic reticulum; $\mathrm{IP}_{3} \mathrm{R} 1$ and $\mathrm{IP}_{3} \mathrm{R} 2$ $-\mathrm{IP}_{3}$ receptor isoforms. 
The parameter values were scaled (to convert from fluxes $(\mu \mathrm{M} / \mathrm{s})$ to currents $(\mathrm{pA})$ ) and modified to generate $\mathrm{Ca}^{2+}$ oscillations in the original $\mathrm{EC}$ model. $\mathrm{IP}_{3}$ activation was also introduced in the CICR current (Equation 4.14). Moreover, the maximum current via $\mathrm{IP}_{3} \mathrm{R}$ in the first pool was set to its initial estimate i.e. $\mathrm{I}_{\mathrm{IP3R}}=1170 \mathrm{pA} / \mathrm{mM}$ (Silva, Kapela, and Tsoukias 2007).

$$
\begin{aligned}
& I_{S E R C A 2}=I_{S E R C A \max }\left(\frac{[C a]_{i}^{2}}{[C a]_{i}^{2}+K_{S E R C A 2}{ }^{2}}\right) \\
& \text { Equation } 4.12 \\
& I_{\text {leak } 2}=K_{\text {leak } 2}\left([\mathrm{Ca}]_{I S 2}-[\mathrm{Ca}]_{i}\right) \\
& \text { Equation } 4.13 \\
& I_{\text {CICR } 2}=I_{\text {CICR } \max }\left(\frac{\left[I P_{3}\right]^{3.8}}{\left[I P_{3}\right]^{3.8}+K 3_{\text {CICR } 2} 3.8}\right)\left(\frac{[C a]_{i}{ }^{4}}{[C a]_{i}{ }^{4}+K 1_{\text {CICR2 }}{ }^{4}}\right)\left(\frac{[C a]_{I S 2}{ }^{2}}{[C a]_{\text {IS2 } 2}{ }^{2}+K 2_{\text {CICR2 }}{ }^{2}}\right)
\end{aligned}
$$

Equation 4.14

The $\mathrm{Ca}^{2+}$ balance in the $2^{\text {nd }}$ pool is described by Equation 4.15 .

$$
\frac{d[C a]_{I S 2}}{d t}=\frac{I_{S E R C A 2}-I_{\text {leak } 2}-I_{C I C R 2}}{Z_{C a} F v o l_{I S 2}}
$$

Equation 4.15

The modified equation for cytosolic calcium change due to incorporation of second pool is given in Equation 4.16.

$$
\frac{d[C a]_{i}}{d t}=\frac{I_{\text {Catotm }}+I_{\text {SERCA }, I S}-I_{\text {leak }, I S}-I_{\text {IP }}+I_{\text {SERCA2 }}-I_{\text {leak } 2}-I_{\text {CICR2 } 2}}{Z_{C a} F v o l_{C a}}-\frac{d[C a]_{b}}{d t}
$$

Equation 4.16

All other model components and parameters are the same as in the previous studies, except 1) Random variations simulating biological noise were implemented; 2) 
Introducing non-uniformity in the store and membrane receptor distribution. Randomized variation with an average gradient in the store and membrane receptor distribution was introduced i.e. on an average the receptors were higher on one side of the cell compared to other as shown in the Figure 4.2-A,4.3-A,4.3-B, 4 4-A,4.7-A and 4.7-B. Gear's backward differential formula method for stiff systems in FORTRAN 90 with maximum time step of $4 \mathrm{~ms}$ was used to solve the system of ordinary differential equations describing the model.

Table 4.1 Parameter values considered in the model

\begin{tabular}{|c|c|c|}
\hline Parameter & Description & Model Value \\
\hline \multicolumn{3}{|c|}{ Diffusivities } \\
\hline$D_{C a}$ & $\begin{array}{l}\text { Diffusion coefficient for } \mathrm{Ca}^{2+} \text { (Allbritton, Meyer, } \\
\text { and Stryer 1992) }\end{array}$ & $300 \mu \mathrm{m}^{2} / \mathrm{s}$ \\
\hline$D_{N a}$ & $\begin{array}{l}\text { Diffusion coefficient for } \mathrm{Na}^{+} \text {(Keener and Sneyd } \\
\text { 1998) }\end{array}$ & $505 \mu \mathrm{m}^{2} / \mathrm{s}$ \\
\hline$D_{K}$ & $\begin{array}{l}\text { Diffusion coefficient for } \mathrm{K}^{+} \text {(Keener and Sneyd } \\
1998 \text { ) }\end{array}$ & $744 \mu \mathrm{m}^{2} / \mathrm{s}$ \\
\hline$D_{C l}$ & $\begin{array}{l}\text { Diffusion coefficient for } \mathrm{Cl}^{-} \text {(Keener and Sneyd } \\
\text { 1998) }\end{array}$ & $900 \mu \mathrm{m}^{2} / \mathrm{s}$ \\
\hline$D_{I P_{3}}$ & $\begin{array}{l}\text { Diffusion coefficient for second messenger } \mathrm{IP}_{3} \\
\text { (Keener and Sneyd 1998) }\end{array}$ & $283 \mu \mathrm{m}^{2} / \mathrm{s}$ \\
\hline \multicolumn{3}{|c|}{ EC Store: $2^{\text {nd }}$ pool paramaters (Parthimos, Edwards, and Griffith 1999) } \\
\hline$[\mathrm{Ca}]_{I S 2}$ & $\begin{array}{l}\text { Initial } \mathrm{Ca}^{2+} \text { concentration in the second pool of } \\
\mathrm{IP}_{3} \text { sensitive store. }\end{array}$ & $0.08 \mathrm{mM}$ \\
\hline$I_{\text {SERCAmax }}$ & $\begin{array}{l}\text { Maximum current via SERCA in second pool of } \\
\mathrm{IP}_{3} \text { sensitive store. }\end{array}$ & $54.7 \mathrm{pA}$ \\
\hline$K_{S E R C A 2}$ & $\begin{array}{l}{\left[\mathrm{Ca}^{2+}\right]_{\mathrm{i}} \text { for half activation of SERCA in second }} \\
\text { pool of IP3-sensitive store. }\end{array}$ & $0.4 \mu \mathrm{M}$ \\
\hline$K_{\text {leak } 2}$ & ER leak constant for second pool & $6.7 \mathrm{pA} / \mathrm{mM}$ \\
\hline$I_{C I C R \max }$ & $\begin{array}{l}\text { Maximum current via CICR channel in second } \\
\text { pool of } \mathrm{IP}_{3} \text { sensitive store. }\end{array}$ & $14850 \mathrm{pA}$ \\
\hline$K 1_{C I C R 2}$ & $\begin{array}{l}{\left[\mathrm{Ca}^{2+}\right]_{i} \text { for half activation of CICR in second }} \\
\text { pool of IP3-sensitive store }\end{array}$ & $0.54 \mu \mathrm{M}$ \\
\hline$K 2_{\text {CICR2 }}$ & $\begin{array}{l}{\left[\mathrm{Ca}^{2+}\right]_{\mathrm{IS} 2} \text { for half activation of CICR } \mathrm{Ca}^{2+} \text { efflux }} \\
\text { in second pool of IP3-sensitive store }\end{array}$ & $120 \mu \mathrm{M}$ \\
\hline$K 3_{\text {CICR2 }}$ & $\begin{array}{l}\mathrm{IP}_{3} \text { concentration for half-maximal CICR } \\
\text { activation }\end{array}$ & $0.5 \mu \mathrm{M}$ \\
\hline vol $_{I S 2}$ & Store volume for the second pool. & $0.28 \mathrm{pL}$ \\
\hline
\end{tabular}


Long simulated times were used (up to 1000 s) to eliminate transient effects. $\mathrm{Ca}^{2+}$ events observed over space and time were plotted as a kymograph i.e. line scans where spatial $\mathrm{Ca}^{2+}$ concentrations are depicted color coded at individual times and are stacked on top of each other to form a 2-D image with $\mathrm{x}$-axis representing cell length and the $\mathrm{y}$-axis representing time and color map showing the $\mathrm{Ca}^{2+}$ concentration at any point on the image. The slopes from the kymograph were used to quantify the $\mathrm{Ca}^{2+}$ wave velocities. Spatial heterogeneity of receptors was also introduced in the 2-D FEM models resulting in oscillatory $\mathrm{Ca}^{2+}$ waves (result shown in supplement (section 4.9)) as in the compartmental model; however all of the results here are from the compartmental model.

\subsection{Results -SMC}

\subsubsection{Oscillatory $\mathrm{Ca}^{2+}$ wave generation}

\subsubsection{Store/Membrane receptor distribution}

Step distribution of adrenergic receptors, through increase in density of receptors in small region (Figure.4.2-A yellow section) and reduced density of receptors in rest of the cell (Figure 4.2-A purple section) to maintain the overall receptor density as in the original model without introduction of the heterogeneity, was introduced in the model. Agonist (280-450 nM) induced $\mathrm{Ca}^{2+}$ elevation within the concentration window for $\mathrm{Ca}^{2+}$ oscillations (Kapela, Bezerianos, and Tsoukias 2008) in the presence of the step distribution of adrenergic receptors (Figure 4.2-A green line) resulted in oscillatory $\mathrm{Ca}^{2+}$ waves. An illustration example of the obtained oscillatory waves at $300 \mathrm{nM}$ agonist concentration is plotted as a kymograph in Figure 4.2-B. To evaluate the role of diffusion of $\mathrm{Ca}^{2+}$ and $\mathrm{IP}_{3}$, and electrical coupling in shaping the oscillatory $\mathrm{Ca}^{2+}$ wave, we blocked 
the inter-compartmental currents for all the species individually and under different combinations. Agonist stimulation of the SMC under the same conditions in Figure.4.2B, with block of inter-compartmental $\mathrm{Ca}^{2+} \operatorname{currents}\left(I_{i c, \mathrm{Ca}}=0\right)$ resulted in loss of oscillatory $\mathrm{Ca}^{2+}$ waves with either individual compartments oscillating out of phase and at different intrinsic frequencies or complete deficiency of oscillations in few or all the compartments (Figure 4.2-C). Mean steady state levels of $\mathrm{Ca}^{2+}, \mathrm{IP}_{3}$ and $\mathrm{V}_{\mathrm{m}}$ potential arising across the cell under different scenarios were recorded and the peak to peak amplitude of their oscillation measured and represented by error bars on the top of the steady state value to visualize the change in levels under oscillations from the mean value.

A)
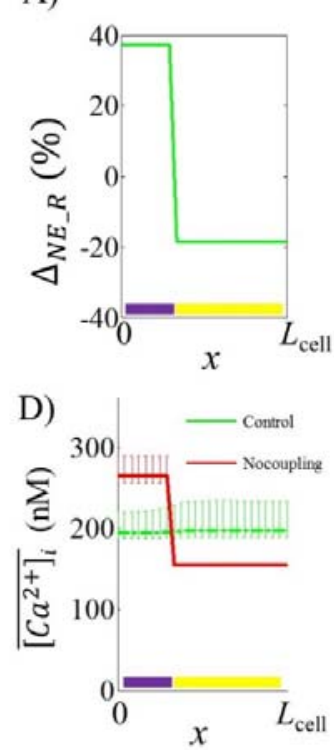

B)

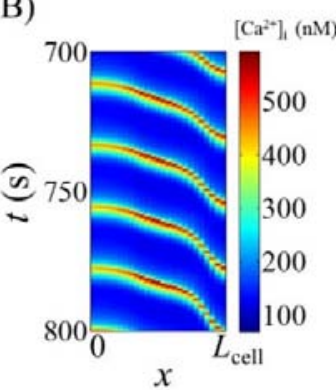

E) 300

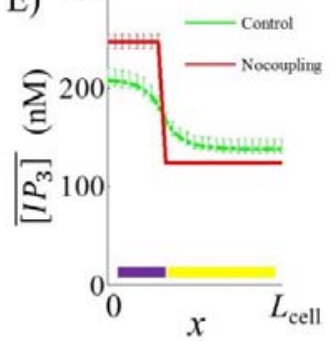

C)
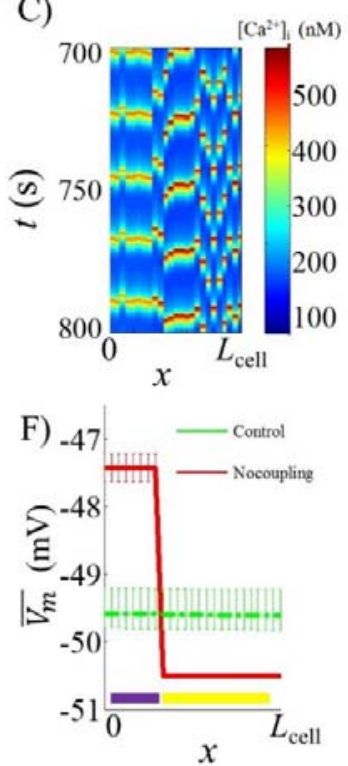

Figure 4.2 (A) Step variation in adrenergic receptors resulting in generation of oscillatory $\mathrm{Ca}^{2+}$ wave in the SMC. (B) $\mathrm{Ca}^{2+}$ concentration amplitude depicted color coded and plotted with time vertically and distance from left to right (Kymograph) arising from step variation of adrenergic receptor distribution, shown in (A). (C) Kymograph of $\mathrm{Ca}^{2+}$ concentration after $\mathrm{Ca}^{2+}$ diffusion block showing the importance of intracellular $\mathrm{Ca}^{2+}$ signaling for maintenance of oscillatory $\mathrm{Ca}^{2+}$ wave (B). Mean $\mathrm{Ca}^{2+}$ concentrations (D), mean $\mathrm{IP}_{3}$ concentrations (E) and mean $V_{\mathrm{m}}$ changes (F) across the cell length arising from adrenergic receptor distribution under control conditions (green line) and block of inter-compartmental currents (red line). The bars represent the amplitude of oscillations in the individual compartments across the cell. 
In the absence of inter-compartmental flux for all species, $\mathrm{Ca}^{2+}$ and $\mathrm{IP}_{3}$ concentrations in the compartments with low density of membrane receptors (Figure 4.2-D and E yellow section of the cell) did not attain sufficient levels (Figure 4.2-D and E red line) to reach the oscillatory window. On the other hand compartments with high density of membrane receptors (Figure 4.2-D and E purple section of the cell) produced $\mathrm{Ca}^{2+}$ and $\mathrm{IP}_{3}$ levels sufficient (Figure 4.2-D and $\mathrm{E}$ red line) for $\mathrm{Ca}^{2+}$ oscillations in those compartments (Figure 4.2-D and E red error bars). Moreover a step change in membrane potential was also observed across the cell (Figure 4.2-F red line) from the block of all intercompartmental fluxes. Such an intervention (uncoupling sections of a cell) is difficult to achieve experimentally but can be easily carried out in the model and will aid us in better understanding the role of these individual species.

Electrical coupling alone (i.e. having the inter-compartmental currents for $\mathrm{Na}^{+}, \mathrm{K}^{+}$and $\mathrm{Cl}^{-}$present) in the absence of inter-compartmental $\mathrm{Ca}^{2+}$ currents resulted in cell becoming iso-potential (uniform mean $V_{\mathrm{m}}$ profile) (Figure 4.2-F green solid line). Oscillatory $\mathrm{Ca}^{2+}$ and $\mathrm{IP}_{3}$ activity was retained in all the individual compartments (by bringing the $\mathrm{Ca}^{2+}$ and $\mathrm{IP}_{3}$ levels in the low receptors density compartments also in the oscillatory window) but no whole cell oscillatory $\mathrm{Ca}^{2+}$ wave was observed (data not shown). Allowing $I_{i c, C a}$ current in addition to electrical coupling synchronized individual compartments to result in formation of whole cell oscillatory waves (Figure 4.2-B). However, it was the oscillatory component of the diffusion $\mathrm{Ca}^{2+}$ current that resulted in synchronization of the individual compartments. The presence/absence of inter-compartmental $\mathrm{IP}_{3}$ currents ( $\left.I_{i c, I P_{3}}\right)$ had no significant effect on the simulations suggesting negligible role for $\mathrm{IP}_{3}$ 
diffusion. The heterogeneity for the wave generation was provided by the $\mathrm{IP}_{3}$ gradient resulting from non-uniform membrane receptor distribution. Figure 4.2-E (green line) shows the gradient in the mean/steady state $\mathrm{IP}_{3}$ concentrations across the cell under control simulation conditions. The steady state $\mathrm{Ca}^{2+}$ levels under control simulation were nearly uniform (Figure 4.2-D green line).

Similar to adrenergic receptors, non-uniform spatial distribution of store (RyR) receptors (Figure 4.3-A and 4.3-B) resulted in oscillatory $\mathrm{Ca}^{2+}$ waves as shown in Figure 4.3-C and 4.3-D. Completely randomized distribution of both membrane and store receptors in the absence of average gradient did not result in generation of an oscillatory $\mathrm{Ca}^{2+}$ wave (result not shown) suggesting the presence of non-uniform spatial distribution of store/membrane receptor with a gradient in receptor density distribution.

A)

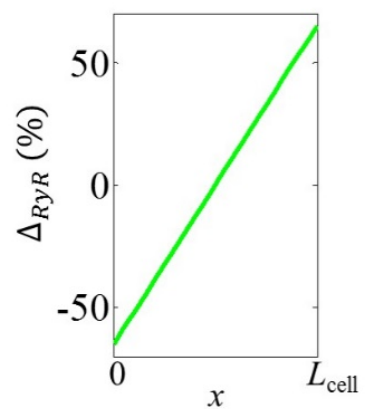

C)

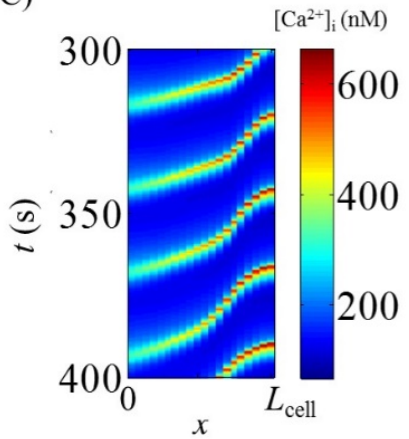

B)

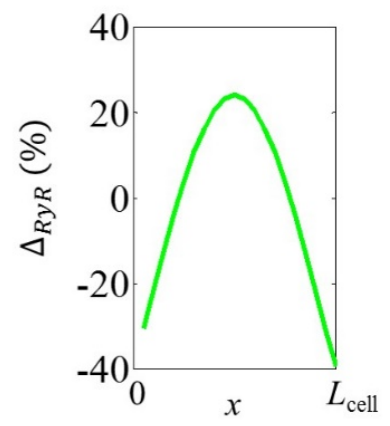

D)

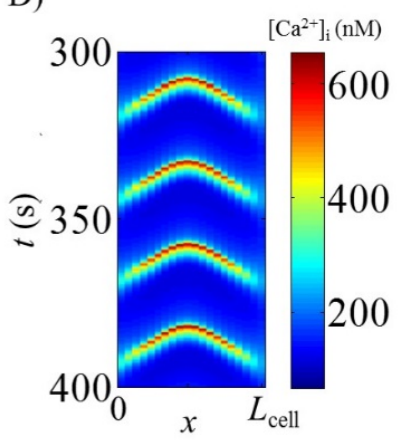

Figure 4.3 Linearized variation in RyR density in (A) and normalized variation in (B) resulted in SMC oscillatory $\mathrm{Ca}^{2+}$ wave's depicted color coded as a kymograph in (C) and (D) respectively. 
However experimental test to validate the presence of such distributions are necessary. The site of initiation of the wave was always the one with the highest density of these receptors. The average slope of distribution of the store and membrane receptors modulated the velocity component of the $\mathrm{Ca}^{2+}$ waves with zero slope (uniform distribution) corresponding to infinite velocities or whole cell oscillations. Increasing the slopes lowered the $\mathrm{Ca}^{2+}$ wave velocity to certain point before which the waves were lost (results not shown).

\subsubsection{Effect of $V_{m}$ dynamics on wave velocity}

Modulation in $V_{\mathrm{m}}$ coupled to $\mathrm{Ca}^{2+}$ oscillations can feedback on the $\mathrm{Ca}^{2+}$ oscillators in individual compartments to modulate $\mathrm{Ca}^{2+}$ wave velocity through $V_{\mathrm{m}}$-dependent $\mathrm{Ca}^{2+}$ channels and alteration of electrochemical gradient for calcium influx. To visualize the influence of $V_{\mathrm{m}}$ dynamics on oscillatory $\mathrm{Ca}^{2+}$ waves we introduced a stimulus current ( $\left.I_{\text {stim }}\right)$ in the model with RyR heterogeneity. Stimulus current forced $V_{\mathrm{m}}$ to follow the

average of calcium levels in all compartments $\left(C a_{o s c}(t)\right)$ with time. The stimulus current forces $V_{\mathrm{m}}$ oscillations $\left(V_{m}(t)\right)$ to be either in phase or anti-phase of $C a_{o s c}(t)$ described by Equation 4.17.

$$
I_{\text {stim }}=K\left( \pm \frac{V_{\max } C a_{o s c}(t)}{1 n M}-\left(V_{m}(t)-\overline{V_{m}}\right)\right)
$$

where

$$
\text { Equation } 4.17
$$

$$
C a_{\text {osc }}(t)=\left(\frac{\sum_{1}^{N} C a_{i}(t)}{N}\right)-\overline{C a_{i}}
$$

Equation 4.18 
is the $\mathrm{AC}$ component of average calcium oscillation in the cell. $V_{m}(t)-\overline{V_{m}}$ is the $\mathrm{AC}$ component of mean $V_{\mathrm{m}}$ oscillation in the cell. $K$ and $V_{\max }$ are the scaling factors that regulate the strength of stimulus and amplitude of $V_{\mathrm{m}}$ oscillation respectively, $N$ is the number of compartments. Both the amplitude of $V_{\mathrm{m}}$ oscillations and its phase with the average $\mathrm{Ca}^{2+}$ concentration $\left(\mathrm{Ca}_{\text {osc }}(t)\right)$ determined the oscillatory $\mathrm{Ca}^{2+}$ wave velocity as shown in Figure 4.4. The wave velocities increased exponentially with the increase in the amplitude of $V_{\mathrm{m}}$ oscillations when $V_{m}(t)$ was made to follow $C a_{o s c}(t)$ in phase $\left(V_{\mathrm{m}}-\mathrm{Ca}^{2+}\right.$ in phase) whereas reduced velocities were observed with increase in amplitude of $V_{\mathrm{m}}$ oscillation when $V_{\mathrm{m}}-\mathrm{Ca}^{2+}$ were anti-phase i.e. increasing the amplitude of $V_{\mathrm{m}}$ oscillations resulted in exponential decrease of wave velocity under this condition.

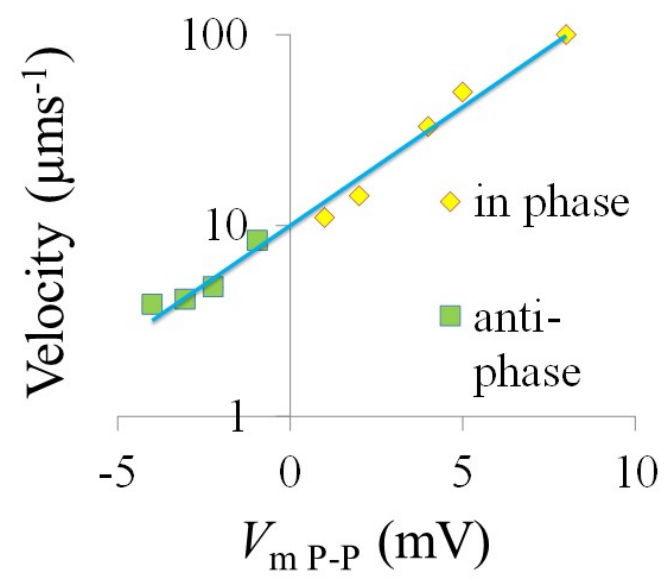

Figure 4.4 Increase in oscillatory $\mathrm{Ca}^{2+}$ wave velocity was observed with increased amplitude of forced $\mathrm{V}_{\mathrm{m}}$ oscillations in phase with the average $\mathrm{Ca}^{2+}$ oscillations while decrease in wave velocities with increased amplitude of forced $\mathrm{V}_{\mathrm{m}}$ oscillations anti-phase to the average $\mathrm{Ca}^{2+}$ oscillations (Negative $V_{\mathrm{m}}$ indicates antiphase with $\mathrm{Ca}^{2+}$ ).

$V_{\mathrm{m}}$ dynamics is modulated by the balance of current through transmembrane channels and pumps. All the model components that influence $V_{\mathrm{m}}$ dynamics were tested to evaluate their role in wave velocity modulation. NE concentration of $280-450 \mathrm{nM}$ under 
step membrane receptor distribution resulted in oscillatory $\mathrm{Ca}^{2+}$ waves with varying velocities. The velocities were calculated from the slope of kymograph and are shown in Figure 4.5-A. Exponentially decreasing velocities were obtained with increase in mean agonist concentration. Clamping of $V_{\mathrm{m}}$ resulted in significant decrease in the wave velocities at low agonist concentration and a relatively smaller change at high agonist levels (Figure 4.5-B). The amplitude of $V_{\mathrm{m}}$ oscillations and its phase with overall intracellular $\mathrm{Ca}^{2+}$ oscillation (the mean of all compartmental $\mathrm{Ca}^{2+}$ concentration at individual time points) in the cell $\left(V_{\mathrm{m}}-\mathrm{Ca}^{2+}\right.$ phase $)$ were recorded and quantified using correlation coefficient.

A)

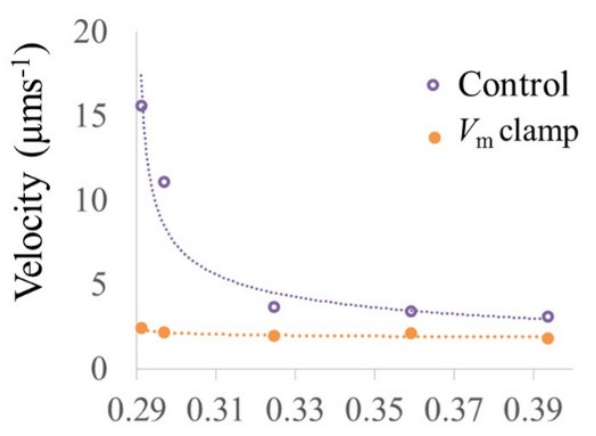

$[\mathrm{NE}](\mu \mathrm{M})$
B)

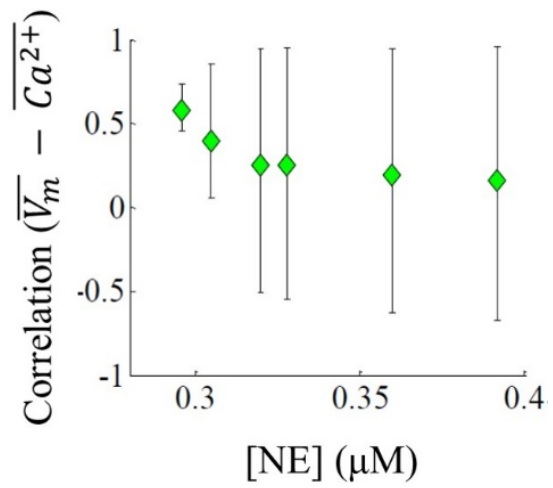

Figure 4.5 (A) Exponential decrease in oscillatory $\mathrm{Ca}^{2+}$ wave velocity with increased agonist $(\mathrm{NE}=0.29-0.4$ $\mu \mathrm{M})$ stimulus in SMC model with NE step variation. (B) Plot of correlation of average $\mathrm{V}_{\mathrm{m}}$ and $\mathrm{Ca}^{2+}$ oscillations against increased NE concentration. Increasing agonist (NE) stimulus resulted in more out of phase $\left(\mathrm{V}_{\mathrm{m}}-\mathrm{Ca}^{2+}\right.$ oscillations). The bars indicate the range of correlation coefficients observed in the individual compartments of the cell and green diamond indicates the mean value of correlation at particular agonist concentration.

The amplitude of $V_{\mathrm{m}}$ oscillations were nearly the same around $2 \mathrm{mV}$ for the range of $\mathrm{NE}$ concentrations. $V_{m}$ and $\mathrm{Ca}^{2+}$ oscillations were in phase (high correlation coefficient) at lower agonist levels. The phase difference started growing with increasing agonist concentrations (reducing correlation coefficient) as shown in Figure 4.5-B. The in-phase 
$V_{\mathrm{m}}-\mathrm{Ca}^{2+}$ at lower agonist concentrations resulted in higher wave velocities compared to out of phase or anti-phase $V_{\mathrm{m}}-\mathrm{Ca}^{2+}$ oscillations at higher agonist concentrations which agrees to prediction by injected stimulus current.

Simulations were performed clamping the individual channels and pumps to their mean steady state currents to analyze the relative importance of each of them in regulation of wave velocities. Clamping NSC channels reduced significantly the amplitude of $V_{\mathrm{m}}$ oscillation from the control (Figure 4.6-A). Simultaneously it resulted in out of phase $V_{\mathrm{m}}-\mathrm{Ca}^{2+}$ oscillations (Figure 4.5-B error bar lower values) resulting in significantly lower wave velocities compared to control at low agonist levels (Figure 4.6$\mathrm{B}$, green triangle). Due to the $\mathrm{Ca}^{2+}$ dependency of the PLC pathway, $\mathrm{Ca}^{2+}$ oscillations generate concomitant oscillations in DAG concentration. Oscillatory DAG in turn modulates NSC channel activation, depolarizes $V_{\mathrm{m}}$, and activates, through electrical coupling, VOCC channels throughout the cell bringing the average $\mathrm{Ca}^{2+}$ oscillations in cell in phase with $V_{\mathrm{m}}$ resulting in increased wave velocities. On the other hand, clamping $\mathrm{BK}_{\mathrm{Ca}}$ channels resulted in significant increase in the amplitude of $V_{\mathrm{m}}$ oscillations (Figure 4.6-A) with higher $V_{\mathrm{m}}-\mathrm{Ca}^{2+}$ correlation (Figure 4.5-B error bar upper values) i.e. in phase $V_{\mathrm{m}}-\mathrm{Ca}^{2+}$ oscillations and consequently significantly higher wave velocities (Figure 4.6-B, purple squares) compared to control. $\mathrm{BK}_{\mathrm{Ca}}$ channels generate hyperpolarizing current in response to $\mathrm{Ca}^{2+}$ elevation. At higher agonist concentrations the $\mathrm{BK}_{\mathrm{Ca}}$ channels are more sensitive to $\mathrm{Ca}^{2+}$ changes compared to NSC in the model and induce $V_{\mathrm{m}}$ hyperpolarization which drive $\mathrm{Ca}^{2+}$ oscillations out of phase with $V_{\mathrm{m}}$. 
The changes in $V_{\mathrm{m}}$ dynamics directly modulate the wave velocities through activation / inactivation of VOCC channels and indirectly by affecting the electrochemical gradient for calcium entry in the cell. Clamping of VOCC channels results in significant reduction in wave velocity at lower range of agonist concentrations as shown in (Figure 4.6-C).

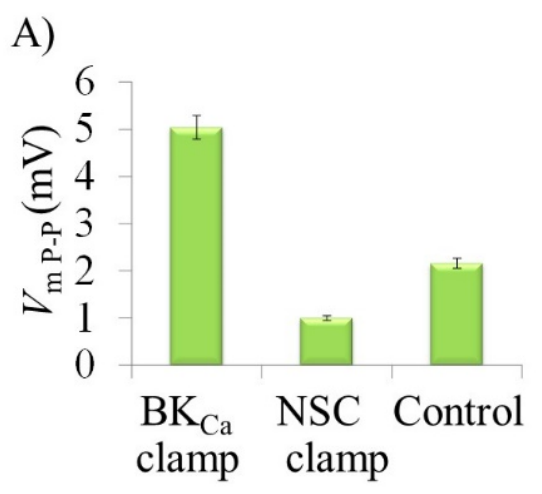

C)

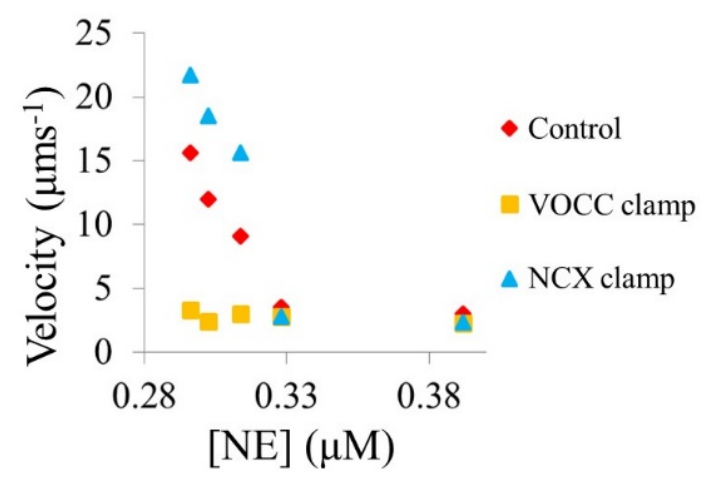

B)

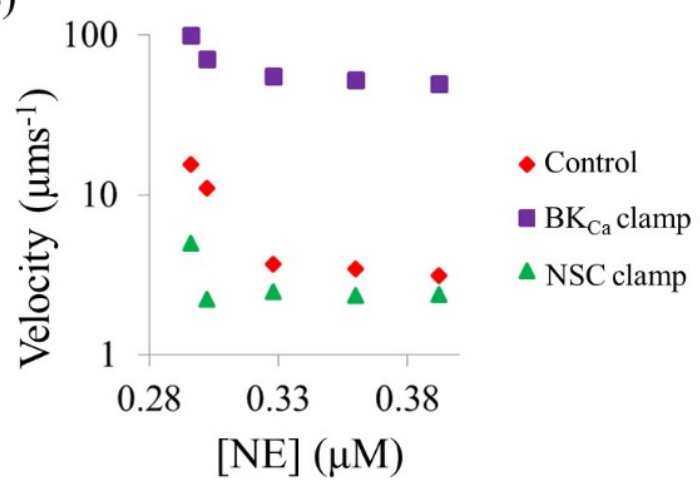

Figure 4.6 In the SMC model with adrenergic receptor step variation (A) clamp of $\mathrm{BK}_{\mathrm{Ca}}$ and NSC channels at different agonist stimulus concentrations $(\mathrm{NE}=0.29-0.4 \mu \mathrm{M})$ resulted in significant increase and decrease respectively in oscillatory $\mathrm{V}_{\mathrm{m}}$ amplitude from the control. Error bars indicate variation in $\mathrm{Vm}(\mathrm{P}-$ P) for varied $\mathrm{NE}$ concentrations $(\mathrm{NE}=0.29-0.4 \mu \mathrm{M})(\mathbf{B})$ The variation in oscillatory $\mathrm{V}_{\mathrm{m}}$ amplitude due to NSC clamp were translated to significant increase in wave velocity due to $\mathrm{BK}_{\mathrm{Ca}}$ clamp and significant decrease in wave velocities under NSC clamp. (C) Clamping VOCC channels resulted in significant decrease in wave velocities at lower range of agonist concentrations but no significant effect at higher agonist concentrations whereas $\mathrm{NCX}\left(\mathrm{Na}^{+}-\mathrm{Ca}^{2+}\right.$ exchanger $)$ resulted in increase in oscillatory wave velocities at lower concentration and a very slight decrease at higher agonist concentrations.

However, at higher concentration of agonist stimulations ( $\mathrm{NE}-0.33-0.39 \mu \mathrm{m}) \mathrm{BK}_{\mathrm{Ca}}$ channels were more dominant resulting in $V_{\mathrm{m}}$ hyperpolarization and no significant effect of VOCC clamp was observed as a result. NCX clamp simulations (Figure 4.6-C) on the 
other hand reveal that at low agonist concentrations it reduces the $\mathrm{Ca}^{2+}$ wave velocities in $V_{\mathrm{m}}$ independent fashion. The flux through NCX depends on sodium concentrations and hence their levels in the cytosol were recorded. In the control simulations $0.5 \mathrm{mM}$ ( $\overline{\left[\mathrm{Na}^{+}\right]_{\mathrm{i}}}=14.70 \mathrm{mM}$ ) amplitude oscillations in $\left[\mathrm{Na}^{+}\right]_{\mathrm{i}}$ were observed. Clamping sodium concentrations in the NCX flux equation to its average value resulted in identical response as NCX current clamp (data not shown) indicating the role of sodium oscillations in generation of the desynchronizing signal through NCX modulation.

\subsection{Results-EC}

\subsubsection{Oscillatory $\mathrm{Ca}^{2+}$ wave generation}

In non-excitable cells (ECs); generation of $\mathrm{Ca}^{2+}$ waves required spatial heterogeneity of membrane / agonist receptors with an overall average gradient, similar to $\mathrm{Ca}^{2+}$ wave production in the SMC model. Agonist stimulation of the EC with muscarinic Ach receptor (Ach_R) step distribution (Figure 4.7-A), through increase in density of receptors in small region (Figure.4.7-A yellow section) and reduced density of receptors in rest of the cell (Figure 4.7-A purple section) to maintain the overall receptor density as in the original model without introduction of the heterogeneity, resulted in generation of oscillatory $\mathrm{Ca}^{2+}$ wave as shown in Figure 4.7-B. Block of inter-compartmental $\mathrm{Ca}^{2+}$ current $\left(I_{i c, C a}\right)$ resulted in loss of oscillatory. $\mathrm{Ca}^{2+}$ wave (Figure 4.7-C) with individual compartments oscillating at random frequencies or complete lack in oscillations. Similar to the SMC model, it was the oscillatory component of $I_{i c, C a}$ current which resulted in synchronization of individual compartments to form the whole cell agonist induced oscillatory waves. Electrical coupling (in the absence of $\mathrm{Ca}^{2+}$ and $\mathrm{IP}_{3}$ inter- 
compartmental currents) resulted in cell becoming iso-potential (Figure 4.7-F green line)

with intrinsic $\mathrm{Ca}^{2+}$ oscillations observed in individual compartments but no whole cell

wave (data not shown).
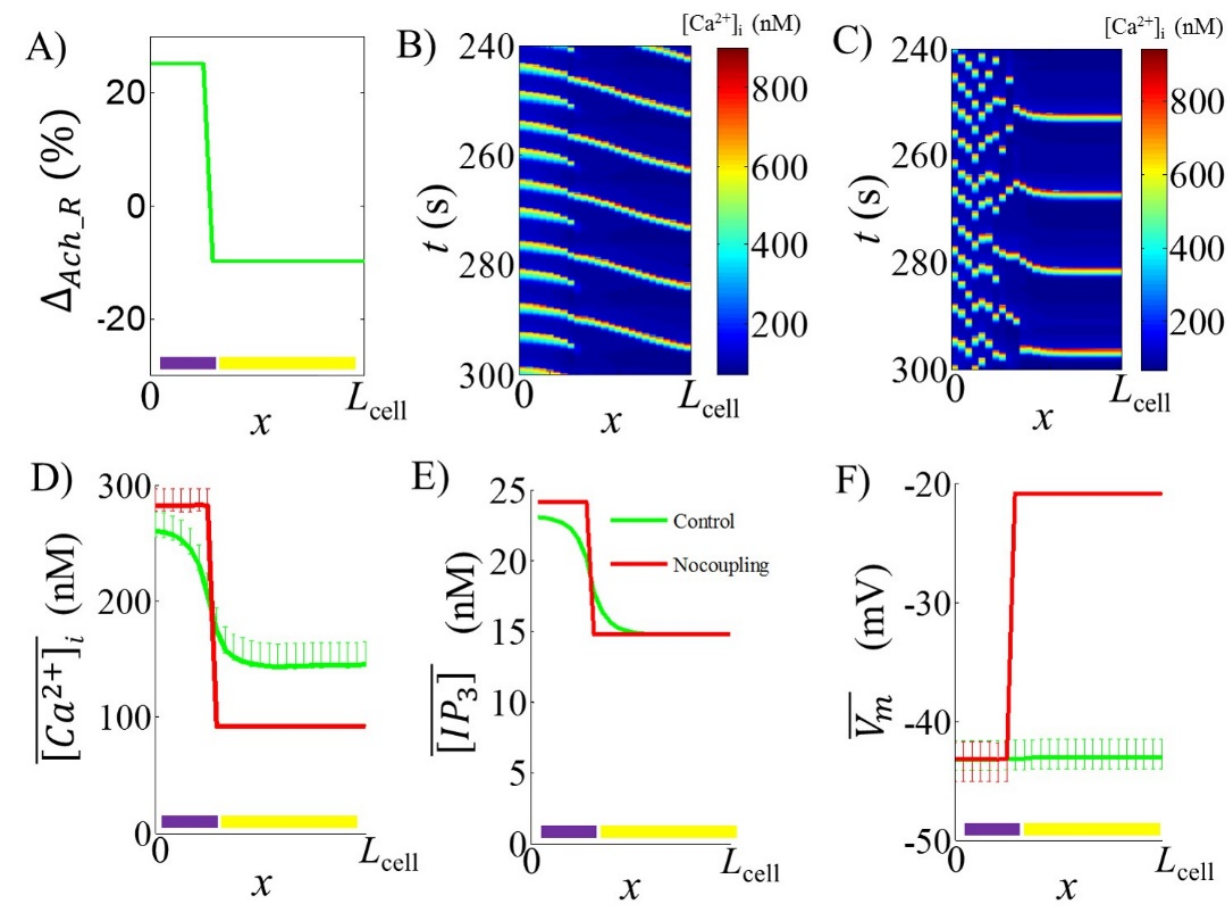

Figure 4.7 (A) Step variation in muscarinic receptors resulting in generation of oscillatory $\mathrm{Ca}^{2+}$ wave in the EC. (B) $\mathrm{Ca}^{2+}$ concentration amplitude depicted color coded and plotted with time vertically and distance from left to right (Kymograph) arising from step variation of muscarinic receptor distribution, shown in (A). (C) Kymograph of $\mathrm{Ca}^{2+}$ concentration after $\mathrm{Ca}^{2+}$ diffusion block showing the importance of intracellular $\mathrm{Ca}^{2+}$ signaling for maintenance of oscillatory $\mathrm{Ca}^{2+}$ wave (B). Mean $\mathrm{Ca}^{2+}$ concentrations (D), mean $\mathrm{IP}_{3}$ concentrations (E) and mean $V_{\mathrm{m}}$ levels $(\mathbf{F})$ across the cell length arising from muscarinic receptor distribution under control conditions (green line) and block of inter-compartmental currents (red line). The bars represent the amplitude of oscillations in the individual compartments across the cell.

In the EC, non-uniform muscarinic receptor density produced both steady state $\mathrm{IP}_{3}$ and $\mathrm{Ca}^{2+}$ concentration gradients (Figure 4.7-D and E) are observed, and provide the necessary spatial heterogeneity for oscillatory $\mathrm{Ca}^{2+}$ wave generation in contrast too only $\mathrm{IP}_{3}$ gradient observed in the SMC model simulations with non-uniform adrenergic receptors. 
Uniform Ach in the presence of gradient in density of IP $_{3}$ Rs (Figure 4.8-A) resulted in oscillatory $\mathrm{Ca}^{2+}$ waves similar to waves obtained under RyR gradients in the SMC.

Oscillatory $\mathrm{Ca}^{2+}$ waves obtained for agonist concentrations of 0.9 and 0.65 (a.u.) are shown in Figure 4.8-B and D respectively. Increase in the frequency of the $\mathrm{Ca}^{2+}$ waves (Figure 4.9-B) was observed at higher agonist concentrations with no significant variation in velocity. $V_{m}$ clamp resulted in reduced wave velocity through modulation of electrochemical gradient for $\mathrm{Ca}^{2+}$ influx.
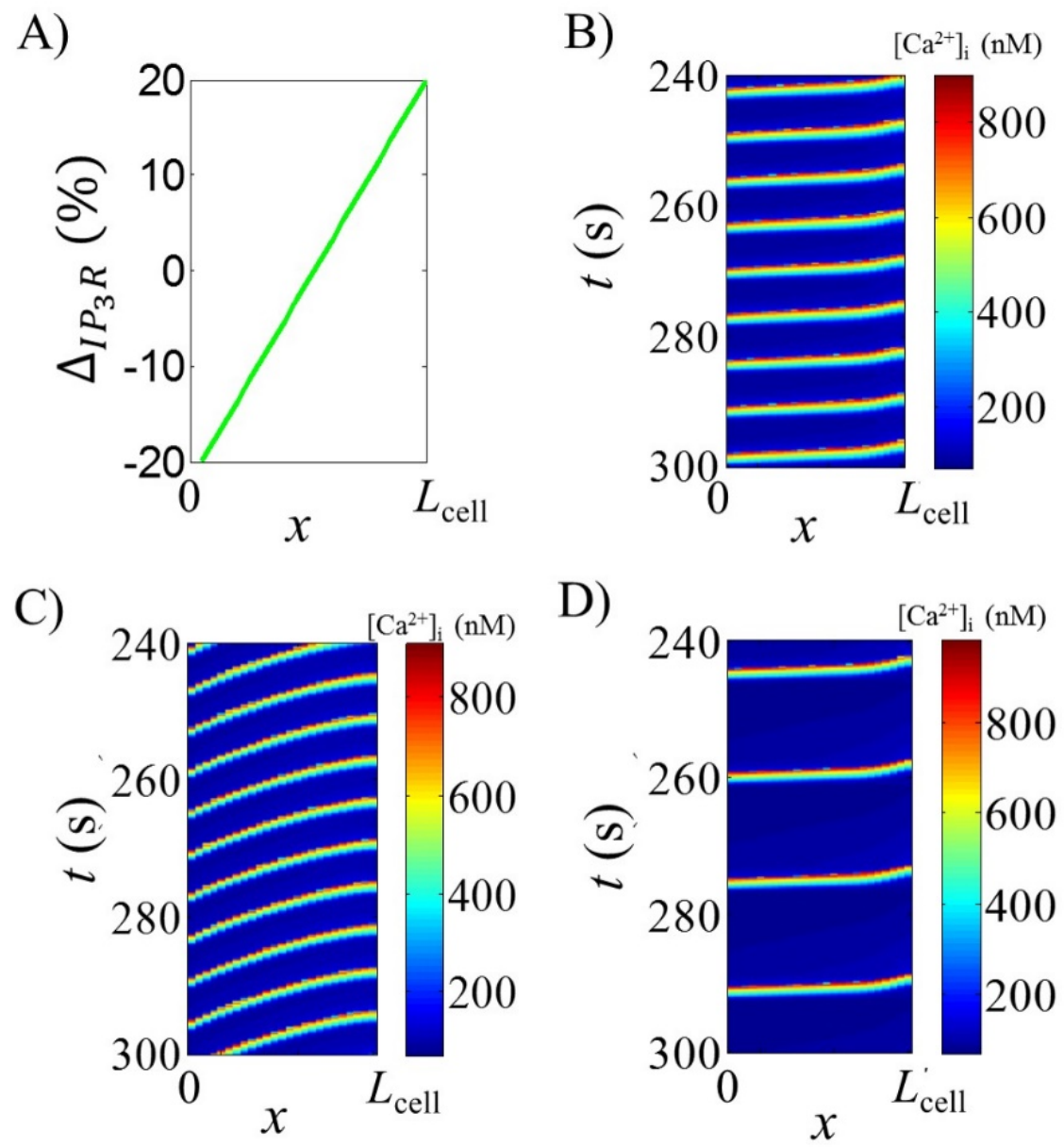

Figure 4.8 (A) Linear variation in $\mathrm{IP}_{3} \mathrm{R}$ density introduced in the model produced oscillatory $\mathrm{Ca}^{2+}$ waves. (B) Kymograph of $\mathrm{Ca}^{2+}$ concentration from stimulus of EC (0.9 a.u. Ach) with linear distribution of $\mathrm{IP}_{3}$ receptors as shown in (A). (C) Kymographs of $\mathrm{Ca}^{2+}$ concentration after oscillatory $\mathrm{V}_{\mathrm{m}}$ clamp with same stimulatory conditions as in (B) reduced the $\mathrm{Ca}^{2+}$ wave velocity due to change in electrochemical gradient from the $\mathrm{V}_{\mathrm{m}}$ clamp. (D) Kymograph of $\mathrm{Ca}^{2+}$ concentration showing the reduction in frequency of oscillatory $\mathrm{Ca}^{2+}$ waves in the EC with reduction in strength of agonist stimulus (0.65 a.u. Ach). 


\subsubsection{Effect of $V_{\mathrm{m}}$ dynamics on wave velocity}

Although not intuitive at first place due to the absence of voltage regulated $\mathrm{Ca}^{2+}$ channels in the EC, our simulations suggests a significant role of $V_{\mathrm{m}}$ dynamics in the regulation of velocity of $\mathrm{EC} \mathrm{Ca}^{2+}$ wave. Simulations with clamped $V_{\mathrm{m}}$ in the $\mathrm{IP}_{3} \mathrm{R}$ gradient model resulted in significant lower wave velocities (Figure 4.8-B, Figure 4.9-A). $V_{\mathrm{m}}$ dynamics in EC are also affected by the relative contribution of currents through individual transmembrane channels and pumps similar to SMC. Agonist (Ach) induced increase in $\mathrm{Ca}^{2+}$ levels in $\mathrm{EC}$ results in activation of $\mathrm{IK}_{\mathrm{Ca}}$ and $\mathrm{SK}_{\mathrm{Ca}}$ channels and hence $V_{\mathrm{m}}$ hyperpolarization. The oscillatory component of $V_{\mathrm{m}}$ dynamics will modulate the electrochemical gradient for $\mathrm{Ca}^{2+}$ influx/efflux via the NSC channels and further the regulation of oscillatory $\mathrm{Ca}^{2+}$ wave velocities.

A)

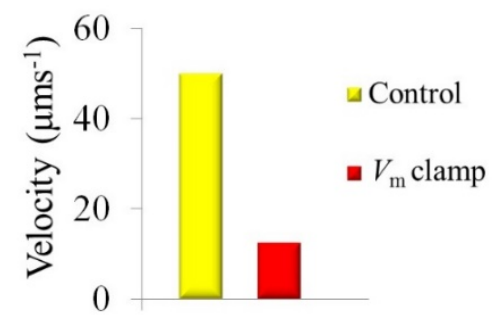

B)

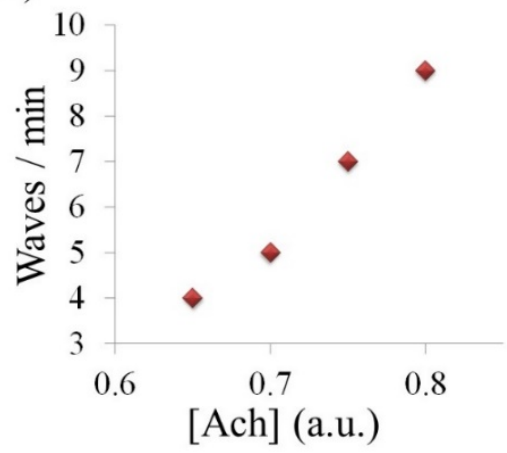

Figure 4.9 stimulation of EC cell with $\mathrm{IP}_{3} \mathrm{R}$ gradient resulted in, (A) Significant reduction in wave velocity (red bar) under $V_{\mathrm{m}}$ clamp condition from control (yellow bar) during. (B) Increase in wave frequency with increase agonist concentration

\subsection{Discussion}

The physiological significance of $\mathrm{Ca}^{2+}$ waves is still unclear however it has been suggested that oscillatory $\mathrm{Ca}^{2+}$ waves cause less contraction than whole-cell $\mathrm{Ca}^{2+}$ oscillations induced by the same agonist concentrations in airway SMC (Wang et al. 
2010). $\mathrm{Ca}^{2+}$ wave blockade in cerebral artery SMC has been shown to reduce the level of myosin regulatory light chain $\left(\mathrm{LC}_{20}\right)$ phosphorylation resulting in dilation (Mufti et al. 2010) however on the contrary it has also been suggested that $\mathrm{Ca}^{2+}$ waves do not contribute significantly to global $\left[\mathrm{Ca}^{2+}\right]_{\mathrm{i}}$ and are therefore not important to force generation in the myogenic response (Jaggar 2001). A possible explanation for these differences is suggested in Cole et al. (Cole and Welsh 2011) which depends on the rate of $\mathrm{LC}_{20}$ dephosphorylation and inter-wave interval. Spatio-temporal $\mathrm{Ca}^{2+}$ wave signaling in the EC can regulate vasomotor tone (Socha et al. 2012) however the exact physiological roles of individual signaling mechanisms still need to be discerned.

\subsubsection{Oscillatory $\mathrm{Ca}^{2+}$ wave generation}

We formulated detailed models for both the EC and SMC to understand the underlying regulatory mechanism for oscillatory $\mathrm{Ca}^{2+}$ wave generation and its modulations. The models predicted the necessity of non-homogeneity of the store and/or membrane receptor distributions for the generation of the oscillatory calcium waves. Specific distributions for these receptors have not yet been reported in literature but their requirement has been suggested in airway smooth muscle cell model (Wang et al. 2010). Higher density of store receptors have been reported near the nucleus in certain cell type's (Vermassen et al. 2003). In the EC layer preparations presence of purinergic/membrane receptors in only $30 \%$ cells have been reported resulting in $\mathrm{Ca}^{2+}$ increase in only one third of the total cells in the absence of gap junctions which increased to $95 \%$ cells in the presence of gap junctions (Kameritsch et al. 2012). The sites with co-localization of Ach receptors and $\mathrm{IP}_{3} \mathrm{R}$ have been suggested to be the site of initiation for the $\mathrm{Ca}^{2+}$ wave propagation in SMC (Olson et al. 2012). Such intra and inter 
cellular variations and presence of microdomains can result in localized regions of cells oscillating at varying frequencies and wave generation through synchronization of these individual oscillators. It can also determine the position of origin of the waves and the length of propagation. In the model the waves always appeared to originate from the site with highest density of the store/membrane receptors (Figure 4.2-A, 4.3-A and B, 4.7-A and 4.8-A). Overall gradient of receptors was necessary to generate oscillatory $\mathrm{Ca}^{2+}$ waves in our model. Completely randomized variation in receptor density resulted in no wave generation. However further validation for the presence of such specific distributions through experimentation is required.

Both $\mathrm{IP}_{3}$ and $\mathrm{Ca}^{2+}$ are shown to play an important role in $\mathrm{Ca}^{2+}$ wave generation and propagation (McCarron et al. 2010). In McCarron et al. elevated IP 3 levels have been suggested to sensitize the $\mathrm{IP}_{3}$ receptors and determines the distance of $\mathrm{Ca}^{2+}$ wave progression. $\mathrm{Ca}^{2+}$ diffusion may induce the propagation through directly acting on store receptors or indirectly through increase in local $\mathrm{IP}_{3}$ levels. Our model suggest that under certain stimulatory conditions electrical coupling in addition to $\mathrm{Ca}^{2+}$ and $\mathrm{IP}_{3}$ might be essential for wave generation (Figure 4.10-A and B). Electrical coupling can drive localized regions of the cell into firing mode by bringing the $\mathrm{Ca}^{2+}$ levels close to oscillatory window. Inter-compartmental oscillatory $\mathrm{Ca}^{2+}$ currents provide synchronizing signals to generate whole cell $\mathrm{Ca}^{2+}$ waves. In addition to sensitization of receptors steady state $\mathrm{Ca}^{2+} / \mathrm{IP}_{3}$ gradient in the model provided the spatial heterogeneity for the oscillatory $\mathrm{Ca}^{2+}$ wave generation. 
A) SMC
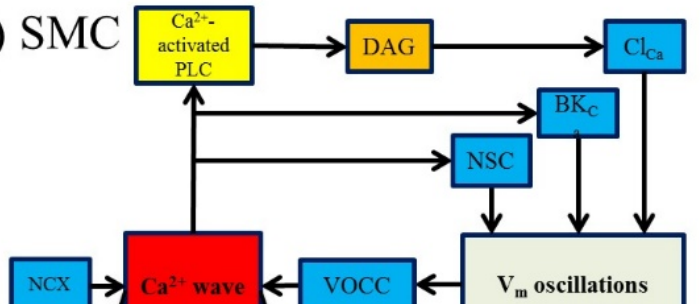

B) $\mathrm{EC}$

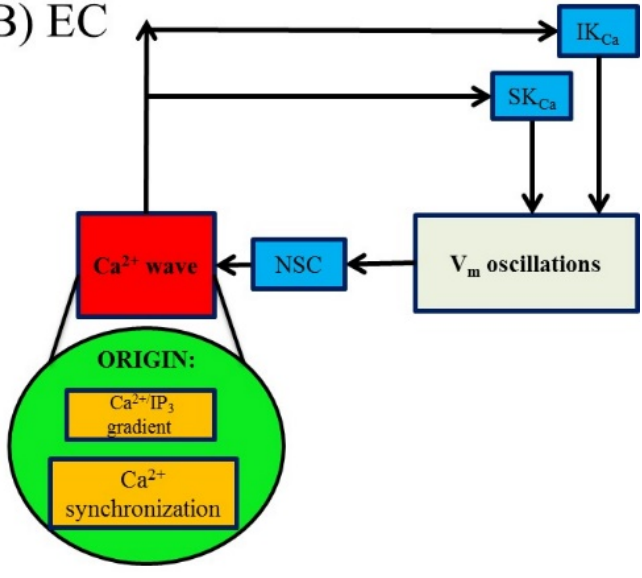

C)

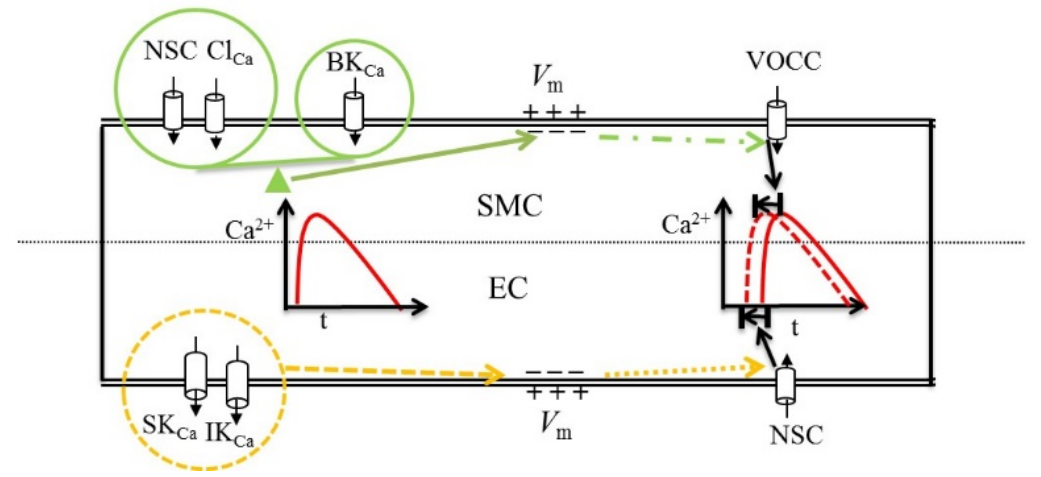

Figure 4.10 Cartoon illustration summarizing the mechanism of generation of oscillatory $\mathrm{Ca}^{2+}$ waves and their modulations in (A) SMC, (B) EC. (C) Phase shift in $\mathrm{Ca}^{2+}$ oscillation's in individual compartments arising from modulation $V_{\mathrm{m}}$ dynamics in the SMC and EC.

\subsubsection{Wave velocities modulation}

A wide range of $\mathrm{Ca}^{2+}$ wave velocities have been reported both in SMC and EC in different experimental condition and cell types (Beliveau, Lapointe, and Guillemette 2011; Huser and Blatter 1997; Jaffe 2010; Narayanan, Adebiyi, and Jaggar 2012). Modulation of wave velocity and frequency with the change in the strength of the agonist stimulus have been shown (Ruehlmann et al. 2000; Socha et al. 2012; Syyong et al. 2009). The underlying mechanisms regulating these changes are unclear yet. In the present study we show that the modulation of the wave velocities may occur through membrane potential $\left(V_{\mathrm{m}}\right)$ dependent pathways. Membrane potential fluctuations regulate 
calcium dynamics directly through voltage regulated L-type calcium channels (VOCC) and indirectly through affecting the electrochemical gradient for calcium influx/efflux in the cell. In endothelial cells no voltage regulated $\mathrm{Ca}^{2+}$ channels has been reported hence direct modulation of $\mathrm{Ca}^{2+}$ in ECs via $V_{\mathrm{m}}$ is not feasible however indirect modulation through changing electrochemical gradient for $\mathrm{Ca}^{2+}$ entry and exit from the cell is possible. Although membrane potential dynamics has been shown to be unimportant for the generation of the $\mathrm{Ca}^{2+}$ wave (Peng et al. 2001) in particular SMCs, it might significantly modulate the already generated waves. In the present model both the amplitude and phase of the membrane potential oscillations with $\mathrm{Ca}^{2+}$ oscillations determines the wave velocity in SMC. Higher amplitude $V_{\mathrm{m}}$ and in-phase $V_{\mathrm{m}}-\mathrm{Ca}^{2+}$ results in whole cells oscillations or higher wave velocities. However out of phase or anti-phase $V_{\mathrm{m}}-\mathrm{Ca}^{2+}$ will result in lower wave velocities. The stimulus strength and relative contributions of depolarizing/hyperpolarizing currents through transmembrane channels and pumps will determine these phase and amplitude of $V_{\mathrm{m}}$ oscillations. In the SMC, $V_{\mathrm{m}}$ oscillations will cause opening/closing of the L-type voltage channels which can speed up or slow down the waves through modulating the $\mathrm{Ca}^{2+}$ entry in the cytosol. VOCC channels blockers have shown to significantly reduce the frequency of $\mathrm{Ca}^{2+}$ waves (Balemba et al. 2006; Dai et al. 2007; Lee et al. 2001) but it does not mean that these channels were directly involved in the frequency modulation as channel block perturbs directly the ionic balance of the cell and the frequency modulation can be indirect consequence of it. Clamping the channel to its average current value gives a better idea of its direct involvement but such experimental intervention is not easy to implement. In the model VOCC clamp resulted in significant decrease in wave velocity at low agonist 
concentrations however it had no impact the frequency of waves. In endothelial cells the voltage regulated $\mathrm{Ca}^{2+}$ channels are absent but $V_{\mathrm{m}}$ oscillations modulates the electrochemical gradient for $\mathrm{Ca}^{2+}$ entry in the cell via NSC channel.

$\mathrm{Cl}_{\mathrm{Ca}}$ channels through depolarization and activation of VOCC have been proposed to bring $\mathrm{Ca}^{2+}$ in phase with $\mathrm{V}_{\mathrm{m}}$ oscillations and conversion from asynchronous waves to whole cell oscillations (Jacobsen et al. 2007). Moreover NSC and $\mathrm{BK}_{\mathrm{Ca}}$ channels have been suggested as important candidates for vasomotion (Kapela, Parikh, and Tsoukias 2012). DAG-activated TRPC-like nonselective cation channels are expressed in RMA SMCs and mediate depolarizing $\mathrm{Na}^{+}$influx during $\alpha_{1}$-adrenergic stimulation (Hill et al. 2006). Block of NSC channels using a non-specific blocker resulted in loss of repetitive wave like $\mathrm{Ca}^{2+}$ oscillations in intact rabbit inferior vena cava (Dai et al. 2007) suggesting its role in maintaining oscillations. Our model predicts NSC channels to be good candidate for modulating wave velocities assuming the DAG oscillations. In the model oscillatory DAG was the result of PLC $\mathrm{Ca}^{2+}$ dependency. Concomitant oscillations in DAG, $\mathrm{IP}_{3}$ and $\mathrm{Ca}^{2+}$ induced by $\mathrm{NE}$ were reported in Chinese hamster ovary cell culture (Bartlett et al. 2005). Recently a robust sensor which can detect DAG and $\mathrm{Ca}^{2+}$ oscillations simultaneously have also been reported (Tewson et al. 2012). Agonist stimulation in the model results in activation of NSC channels resulting in highly correlated average $V_{\mathrm{m}}-\mathrm{Ca}^{2+}$ oscillations and hence higher wave velocities. However, the $\mathrm{BK}_{\mathrm{Ca}}$ channels counteract the NSC channels and depending on the agonist concentration and dominance of these channels the amplitude and phase of $V_{\mathrm{m}}$ oscillations with respect to $\mathrm{Ca}^{2+}$ oscillations is maintained and significant variation in wave velocities could be observed. A summary figure highlighting the chief components 
involved in generation and modulation of oscillatory $\mathrm{Ca}^{2+}$ waves in both $\mathrm{EC}$ and SMC are shown in Figure 4.10-A and B. Figure 4.10-C illustrates the role of $V_{\mathrm{m}}$ dynamics in both excitable and non-excitable cells in modulation of oscillatory $\mathrm{Ca}^{2+}$ velocities and exhibits the main difference between the two cell types for cytosolic $\mathrm{Ca}^{2+}$ level modulation. In excitable cells depolarization of $V_{\mathrm{m}}(\mathrm{SMC})$ will result in increase in $\mathrm{Ca}^{2+}$ levels via entry through voltage regulated $\mathrm{Ca}^{2+}$ channels whereas in non-excitable cells it is the hyperpolarization of $V_{\mathrm{m}}$ that will result in increase in $\mathrm{Ca}^{2+}$ levels through modulation of electrochemical gradient for more $\mathrm{Ca}^{2+}$ influx.

NCX blockers have been reported to abolish repetitive calcium waves and significantly reduce tonic contraction through $\mathrm{Ca}^{2+}$ entry block in SMC (Lee et al. 2001). Block of any channel can significantly modulate the system over all and the resultant change cannot be attributed to that particular channel. In the SMC model NCX clamp produced a desynchronizing signal due to $\left[\mathrm{Na}^{+}\right]_{\mathrm{i}}$ oscillations resulting in reduction of wave velocities at lower agonist concentrations through a $V_{\mathrm{m}}$ independent pathway. At higher agonist concentrations $\mathrm{BK}_{\mathrm{Ca}}$ being more dominant results in no significant effect of NCX.

\subsection{Model limitations}

Due to lack of relevant experimental data and computational limitation several necessary simplifying assumptions were carried out in the model. The underlying mechanism for $\mathrm{Ca}^{2+}$ oscillation in EC and SMC have not been resolved definitively with lack of any models for $\mathrm{Ca}^{2+}$ oscillations in $\mathrm{EC}$. Both $\mathrm{IP}_{3}$ and RyR individually and together have been suggested to be the main components for $\mathrm{Ca}^{2+}$ oscillations. In this study regular $\mathrm{Ca}^{2+}$ oscillations are generated by RyRs and slow refilling in SR for SMC 
as in (Koenigsberger et al. 2004) and with a help of a novel two pool model with oscillations generated by $\mathrm{IP}_{3} \mathrm{Rs}$ and slow refilling in SR for EC as in (PaltaufDoburzynska et al. 2000). We account for non-homogenous distributions of RyRs and $\mathrm{IP}_{3}$ Rs and membrane receptors however their specific distributions have not been reported experimentally hence the choice of these distributions were take on arbitrarily. Compartmentalization of certain channels for generation of $\mathrm{Ca}^{2+}$ sparks, puffs, sparklets have been reported in the literature but not incorporated here which may have a significant effect on model predictions. Moreover the current model is deterministic however the stochastic opening of individual channels have been reported and suggested to play an important role however the current model does not account for it.

\subsection{Conclusion}

The model predicts the presence of non -homogenous distribution of store/membrane receptors for the generation of oscillatory $\mathrm{Ca}^{2+}$ waves in both $\mathrm{EC}$ and SMC. It suggests the requirement of steady state $\mathrm{IP}_{3 /} \mathrm{Ca}^{2+}$ gradient and oscillatory $\mathrm{Ca}^{2+}$ diffusion for origin and propagation of oscillatory $\mathrm{Ca}^{2+}$ waves. Moreover it explains the role of electrical coupling $/ V_{\mathrm{m}}$ dynamics in the generation and modulation of $\mathrm{Ca}^{2+}$ waves. Stimulatory conditions and relative channel contributions will determine the speed and frequency of the oscillatory $\mathrm{Ca}^{2+}$ waves. The model also reveals the presence of several physiologically feasible pathways for modulation of wave velocities. This may explain inconsistent experimental results for generation of oscillatory $\mathrm{Ca}^{2+}$ wave and its regulation. 


\subsection{Supplement}

\subsubsection{Compartmental model for oscillatory $\mathrm{Ca}^{2+}$ wave}

The single cell models of EC and SMC were discretized into $\mathrm{N}=200$ compartments with each compartment incorporating the cellular components as in the original models. All the fluxes in the individual compartments were scaled by $\mathrm{N}$ (total number of compartments) and the compartments were coupled by the electro-diffusion equations (Nagaraja, Kapela, and Tsoukias 2012) as described in Equation 4.19

$$
\mathrm{I}_{\mathrm{ic}, \mathrm{S}}=P_{i c, S} z_{S} F\left(\Delta[S]_{i c}+\frac{z_{S} F}{R T}[\bar{S}]_{i c} \Delta V_{i c}\right)
$$

Equation 4.19

where $z_{S}, F R$ and $T$ are the valence of ion $S$, Faraday's constant, gas constant and temperature, respectively, $I_{i c, S}$ is the inter-compartmental flux of the species $\mathrm{S}, \Delta[S]_{i c}=$ $[S]^{n}-[S]^{m}$, and $[\bar{S}]_{\text {ic }}=\left([S]^{n}-[S]^{m}\right) / 2$ is the average concentration across the compartments $m$ and n. $\Delta[V]_{i c}=[V]^{n}-[V]^{m}$ is the change in membrane potential across the compartments $\mathrm{m}$ and $\mathrm{n}, P_{i c, S}$ is the inter-compartmental permeability to $\mathrm{S}$. The permeability's for each of the ions $\left(\mathrm{Ca}^{2+}, \mathrm{Cl}^{-}, \mathrm{Na}^{+}\right.$and $\left.\mathrm{K}^{+}\right)$and second messenger $\left(\mathrm{IP}_{3}\right)$ were calculated by:

$$
P_{i c, S}=\frac{D_{S} A}{L / N}
$$

Equation 4.20

where $A, L, N$ are cross sectional area of cell, length of cell and number of compartments in the cell respectively. $D_{S}$ is the intra-cellular diffusion coefficient's for species $\mathrm{S}$ where $\mathrm{S}$ is $\mathrm{Ca}^{2+}, \mathrm{K}^{+}, \mathrm{Na}^{+}, \mathrm{Cl}^{-}$and $\mathrm{IP}_{3}$ and their respective values are 
300(Allbritton, Meyer, and Stryer 1992), 744(Allbritton, Meyer, and Stryer 1992), 505(Keener and Sneyd 1998), 900(Keener and Sneyd 1998) and 283(Keener and Sneyd 1998).

Oscillatory $\mathrm{Ca}^{2+}$ wave obtained under control conditions in SMC with $\mathrm{N}=200$ is shown in Figure 4.11-A. However $\mathrm{N}=25$ resulted in similar results to discretization into 200 compartments and hence all the further simulations were carried using $\mathrm{N}=25$ to reduce computational overheads.

\subsubsection{Finite element model (FEM) for oscillatory $\mathrm{Ca}^{2+}$ wave}

FEM models provide Continuous 2-D spatial resolution within cytosol and microdomains. They will enable us to study localized events such as $\mathrm{Ca}^{2+}$ sparks and puffs or intra- and inter-cellular $\mathrm{Ca}^{2+}$ waves, which are now recognized as important aspects of $\mathrm{Ca}^{2+}$ signaling. We have implemented EC and SMC models on 2-D rectangular domains and cylindrical domain considering axial symmetry using COMSOL Multiphysics software (Kapela and Tsoukias 2011).

A)

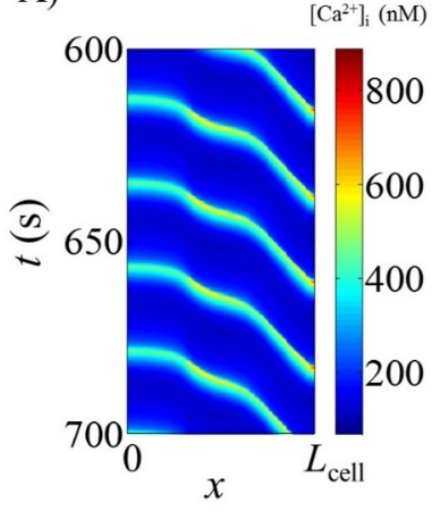

B)

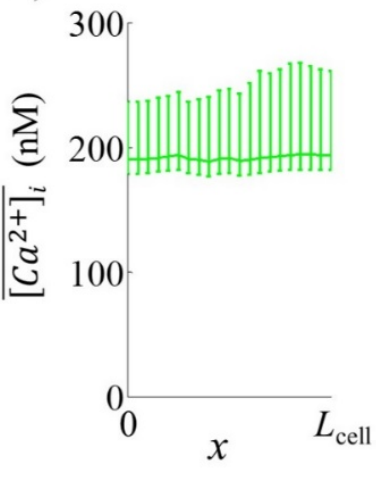

C)

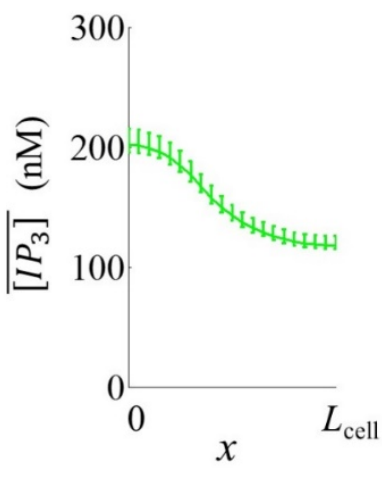

Figure 4.11 (A)Agonist (300 nM) induced oscillatory Ca2+ wave in the SMC under step distribution of adrenergic receptors, obtained using discretized compartmental model ( $\mathrm{N}=200$ compartments) Mean steady state $\mathrm{Ca}^{2+}$ profiles $(\mathbf{B})$ and $\mathrm{IP}_{3}$ profile in $(\mathbf{C})$ across the cell in 2-D FEM SMC model under agonist (300 $\mathrm{nM})$. Bars indicated the amplitude of oscillations 
Electrodiffusion equations with the electroneutrality condition were used to calculate intracellular and extracellular concentrations of ionic species $S=\mathrm{Ca}^{2+}, \mathrm{Na}^{+}, \mathrm{K}^{+}, \mathrm{Cl}^{-}$. The voltage and concentration dependent membrane channel currents were implemented as boundary conditions. The details of the model is described in Kapela et al. 2011 (Kapela and Tsoukias 2011). The models were modified to introduce spatial distribution of membrane/store receptors similar to the compartmental model. The oscillatory $\mathrm{Ca}^{2+}$ wave obtained from the FEM model were similar to compartmental model. The mean profiles of $\mathrm{Ca}^{2+}$ and $\mathrm{IP}_{3}$ obtained from the FEM model are as shown in Figure 4.11-B and C. They are similar to the mean profiles obtained from the compartmental model (Figure 4.2)

\subsection{References}

Allbritton, N. L., T. Meyer, and L. Stryer. 1992. Range of messenger action of calcium ion and inositol 1,4,5-trisphosphate. Science 258 (5089):1812-5.

Amberg, G. C., and M. F. Navedo. 2013. Calcium dynamics in vascular smooth muscle. Microcirculation 20 (4):281-9.

Atri, A., J. Amundson, D. Clapham, and J. Sneyd. 1993. A single-pool model for intracellular calcium oscillations and waves in the Xenopus laevis oocyte. Biophys $J 65$ (4):1727-39.

Bai, Y., M. Edelmann, and M. J. Sanderson. 2009. The contribution of inositol 1,4,5trisphosphate and ryanodine receptors to agonist-induced $\mathrm{Ca}(2+)$ signaling of airway smooth muscle cells. Am J Physiol Lung Cell Mol Physiol 297 (2):L34761.

Balemba, O. B., T. J. Heppner, A. D. Bonev, M. T. Nelson, and G. M. Mawe. 2006. Calcium waves in intact guinea pig gallbladder smooth muscle cells. Am J Physiol Gastrointest Liver Physiol 291 (4):G717-27.

Bartlett, P. J., K. W. Young, S. R. Nahorski, and R. A. Challiss. 2005. Single cell analysis and temporal profiling of agonist-mediated inositol 1,4,5-trisphosphate, $\mathrm{Ca} 2+$, diacylglycerol, and protein kinase $\mathrm{C}$ signaling using fluorescent biosensors. $J$ Biol Chem 280 (23):21837-46. 
Beliveau, E., F. Lapointe, and G. Guillemette. 2011. The activation state of the inositol 1,4,5-trisphosphate receptor regulates the velocity of intracellular $\mathrm{Ca} 2+$ waves in bovine aortic endothelial cells. J Cell Biochem 112 (12):3722-31.

Cole, W. C., and D. G. Welsh. 2011. Role of myosin light chain kinase and myosin light chain phosphatase in the resistance arterial myogenic response to intravascular pressure. Arch Biochem Biophys 510 (2):160-73.

Dai, J., C. H. Lee, D. Poburko, T. Szado, K. H. Kuo, and C. van Breemen. 2007. Endothelin-1-mediated wave-like [Ca2+]i oscillations in intact rabbit inferior vena cava. J Vasc Res 44 (6):495-503.

Dai, J. M., K. H. Kuo, J. M. Leo, C. van Breemen, and C. H. Lee. 2006. Mechanism of $\mathrm{ACh}$-induced asynchronous calcium waves and tonic contraction in porcine tracheal muscle bundle. Am J Physiol Lung Cell Mol Physiol 290 (3):L459-69.

Dupont, G., and A. Goldbeter. 1994. Properties of intracellular Ca2+ waves generated by a model based on $\mathrm{Ca}(2+)$-induced Ca2+ release. Biophys $J 67$ (6):2191-204.

Falcke, M. 2003. On the role of stochastic channel behavior in intracellular $\mathrm{Ca} 2+$ dynamics. Biophys $J 84$ (1):42-56.

Falcke, M., M. Or-Guil, and M. Bar. 2000. Dispersion gap and localized spiral waves in a model for intracellular Ca2+ dynamics. Phys Rev Lett 84 (20):4753-6.

Falcke, M., L. Tsimring, and H. Levine. 2000. Stochastic spreading of intracellular $\mathrm{Ca}(2+)$ release. Phys Rev E Stat Phys Plasmas Fluids Relat Interdiscip Topics 62 (2 Pt B):2636-43.

Foskett, J. K., C. White, K. H. Cheung, and D. O. Mak. 2007. Inositol trisphosphate receptor Ca2+ release channels. Physiol Rev 87 (2):593-658.

Halidi, N., F. X. Boittin, J. L. Beny, and J. J. Meister. 2011. Propagation of fast and slow intercellular $\mathrm{Ca}(2+)$ waves in primary cultured arterial smooth muscle cells. Cell Calcium 50 (5):459-67.

Hill, A. J., J. M. Hinton, H. Cheng, Z. Gao, D. O. Bates, J. C. Hancox, P. D. Langton, and A. F. James. 2006. A TRPC-like non-selective cation current activated by alpha 1adrenoceptors in rat mesenteric artery smooth muscle cells. Cell Calcium 40 (1):29-40.

Huser, J., and L. A. Blatter. 1997. Elementary events of agonist-induced Ca2+ release in vascular endothelial cells. Am J Physiol 273 (5 Pt 1):C1775-82.

Isshiki, M., J. Ando, R. Korenaga, H. Kogo, T. Fujimoto, T. Fujita, and A. Kamiya. 1998. Endothelial $\mathrm{Ca} 2+$ waves preferentially originate at specific loci in caveolin-rich cell edges. Proc Natl Acad Sci US A 95 (9):5009-14. 
Jacobsen, J. C., C. Aalkjaer, H. Nilsson, V. V. Matchkov, J. Freiberg, and N. H. HolsteinRathlou. 2007. Activation of a cGMP-sensitive calcium-dependent chloride channel may cause transition from calcium waves to whole cell oscillations in smooth muscle cells. Am J Physiol Heart Circ Physiol 293 (1):H215-28.

Jaffe, L. F. 2010. Fast calcium waves. Cell Calcium 48 (2-3):102-13.

Jaggar, J. H. 2001. Intravascular pressure regulates local and global $\mathrm{Ca}(2+)$ signaling in cerebral artery smooth muscle cells. Am J Physiol Cell Physiol 281 (2):C439-48.

Kameritsch, P., K. Pogoda, A. Ritter, S. Munzing, and U. Pohl. 2012. Gap junctional communication controls the overall endothelial calcium response to vasoactive agonists. Cardiovasc Res 93 (3):508-15.

Kapela, A., A. Bezerianos, and N. M. Tsoukias. 2008. A mathematical model of Ca2+ dynamics in rat mesenteric smooth muscle cell: agonist and NO stimulation. $J$ Theor Biol 253 (2):238-60.

Kapela, A., J. Parikh, and N. M. Tsoukias. 2012. Multiple factors influence calcium synchronization in arterial vasomotion. Biophys $J 102$ (2):211-20.

Kapela, A., and N. M. Tsoukias. 2011. Multiscale FEM modeling of vascular tone: from membrane currents to vessel mechanics. IEEE Trans Biomed Eng 58 (12):3456-9.

Keener, James P., and James Sneyd. 1998. Mathematical physiology, Interdisciplinary applied mathematics. New York: Springer.

Koenigsberger, M., R. Sauser, M. Lamboley, J. L. Beny, and J. J. Meister. 2004. Ca2+ dynamics in a population of smooth muscle cells: modeling the recruitment and synchronization. Biophys $J 87$ (1):92-104.

Lagaud, G. J., V. Randriamboavonjy, G. Roul, J. C. Stoclet, and R. Andriantsitohaina. 1999. Mechanism of $\mathrm{Ca} 2+$ release and entry during contraction elicited by norepinephrine in rat resistance arteries. Am J Physiol 276 (1 Pt 2):H300-8.

Lamont, C., and W. G. Wier. 2004. Different roles of ryanodine receptors and inositol $(1,4,5)$-trisphosphate receptors in adrenergically stimulated contractions of small arteries. Am J Physiol Heart Circ Physiol 287 (2):H617-25.

Ledoux, J., M. S. Taylor, A. D. Bonev, R. M. Hannah, V. Solodushko, B. Shui, Y. Tallini, M. I. Kotlikoff, and M. T. Nelson. 2008. Functional architecture of inositol 1,4,5-trisphosphate signaling in restricted spaces of myoendothelial projections. Proc Natl Acad Sci U S A 105 (28):9627-32.

Lee, C. H., D. Poburko, P. Sahota, J. Sandhu, D. O. Ruehlmann, and C. van Breemen. 2001. The mechanism of phenylephrine-mediated $[\mathrm{Ca}(2+)](\mathrm{i})$ oscillations 
underlying tonic contraction in the rabbit inferior vena cava. $J$ Physiol $534(\mathrm{Pt}$ 3):641-50.

Marchant, J., N. Callamaras, and I. Parker. 1999. Initiation of IP(3)-mediated $\mathrm{Ca}(2+)$ waves in Xenopus oocytes. EMBO J 18 (19):5285-99.

McCarron, J. G., S. Chalmers, D. MacMillan, and M. L. Olson. 2010. Agonist-evoked $\mathrm{Ca}(2+)$ wave progression requires $\mathrm{Ca}(2+)$ and IP(3). J Cell Physiol 224 (2):33444.

Mufti, R. E., S. E. Brett, C. H. Tran, R. Abd El-Rahman, Y. Anfinogenova, A. El-Yazbi, W. C. Cole, P. P. Jones, S. R. Chen, and D. G. Welsh. 2010. Intravascular pressure augments cerebral arterial constriction by inducing voltage-insensitive Ca2+ waves. $J$ Physiol 588 (Pt 20):3983-4005.

Nagaraja, S., A. Kapela, and N. M. Tsoukias. 2012. Intercellular communication in the vascular wall: a modeling perspective. Microcirculation 19 (5):391-402.

Narayanan, D., A. Adebiyi, and J. H. Jaggar. 2012. Inositol trisphosphate receptors in smooth muscle cells. Am J Physiol Heart Circ Physiol 302 (11):H2190-210.

Olson, M. L., M. E. Sandison, S. Chalmers, and J. G. McCarron. 2012. Microdomains of muscarinic acetylcholine and Ins $(1,4,5) \mathrm{P} 3$ receptors create 'Ins $(1,4,5) \mathrm{P} 3$ junctions' and sites of $\mathrm{Ca} 2+$ wave initiation in smooth muscle. J Cell Sci 125 (Pt 22):531528.

Paltauf-Doburzynska, J., M. Frieden, M. Spitaler, and W. F. Graier. 2000. Histamineinduced $\mathrm{Ca} 2+$ oscillations in a human endothelial cell line depend on transmembrane ion flux, ryanodine receptors and endoplasmic reticulum $\mathrm{Ca} 2+-$ ATPase. J Physiol 524 Pt 3:701-13.

Parthimos, D., D. H. Edwards, and T. M. Griffith. 1999. Minimal model of arterial chaos generated by coupled intracellular and membrane $\mathrm{Ca} 2+$ oscillators. Am J Physiol 277 (3 Pt 2):H1119-44.

Peng, H., V. Matchkov, A. Ivarsen, C. Aalkjaer, and H. Nilsson. 2001. Hypothesis for the initiation of vasomotion. Circ Res 88 (8):810-5.

Postnov, D. E., J. C. Jacobsen, N. H. Holstein-Rathlou, and O. V. Sosnovtseva. 2011. Functional modeling of the shift in cellular calcium dynamics at the onset of synchronization in smooth muscle cells. Bull Math Biol 73 (10):2507-25.

Ruehlmann, D. O., C. H. Lee, D. Poburko, and C. van Breemen. 2000. Asynchronous $\mathrm{Ca}(2+)$ waves in intact venous smooth muscle. Circ Res 86 (4):E72-9. 
Seppey, D., R. Sauser, M. Koenigsberger, J. L. Beny, and J. J. Meister. 2010. Intercellular calcium waves are associated with the propagation of vasomotion along arterial strips. Am J Physiol Heart Circ Physiol 298 (2):H488-96.

Silva, H. S., A. Kapela, and N. M. Tsoukias. 2007. A mathematical model of plasma membrane electrophysiology and calcium dynamics in vascular endothelial cells. Am J Physiol Cell Physiol 293 (1):C277-93.

Sneyd, J., B. T. Wetton, A. C. Charles, and M. J. Sanderson. 1995. Intercellular calcium waves mediated by diffusion of inositol trisphosphate: a two-dimensional model. Am J Physiol 268 (6 Pt 1):C1537-45.

Socha, M. J., T. L. Domeier, E. J. Behringer, and S. S. Segal. 2012. Coordination of intercellular ca $(2+)$ signaling in endothelial cell tubes of mouse resistance arteries. Microcirculation 19 (8):757-70.

Syyong, H. T., H. H. Yang, G. Trinh, C. Cheung, K. H. Kuo, and C. van Breemen. 2009. Mechanism of asynchronous $\mathrm{Ca}(2+)$ waves underlying agonist-induced contraction in the rat basilar artery. Br J Pharmacol 156 (4):587-600.

Tewson, P., M. Westenberg, Y. Zhao, R. E. Campbell, A. M. Quinn, and T. E. Hughes. 2012. Simultaneous detection of $\mathrm{Ca} 2+$ and diacylglycerol signaling in living cells. PLoS One 7 (8):e42791.

Vermassen, E., K. Van Acker, W. G. Annaert, B. Himpens, G. Callewaert, L. Missiaen, H. De Smedt, and J. B. Parys. 2003. Microtubule-dependent redistribution of the type-1 inositol 1,4,5-trisphosphate receptor in A7r5 smooth muscle cells. $J$ Cell Sci 116 (Pt 7):1269-77.

Wang, I. Y., Y. Bai, M. J. Sanderson, and J. Sneyd. 2010. A mathematical analysis of agonist- and $\mathrm{KCl}$-induced $\mathrm{Ca}(2+)$ oscillations in mouse airway smooth muscle cells. Biophys $J 98$ (7):1170-81. 


\section{CHAPTER 5-SYNCHRONIZATION OF CALCIUM OSCILLATIONS IN}

VASCULAR SMOOTH MUSCLE CELLS

This chapter is published in Biophysical Journal (with slight modifications) as A.

Kapela , Parikh, J,and N. M.Tsoukias. "Multiple Factors Influence Calcium Synchronization in Arterial Vasomotion" 


\subsection{Abstract}

The intercellular synchronization of spontaneous calcium $\left(\mathrm{Ca}^{2+}\right)$ oscillations in individual smooth muscle cells (SMC) is a prerequisite for vasomotion. A detailed mathematical model of $\mathrm{Ca}^{2+}$ dynamics in rat mesenteric arteries shows that a number of synchronizing and desynchronizing pathways may be involved. In particular, $\mathrm{Ca}^{2+}$ dependent phospholipase $\mathrm{C}$, the intercellular diffusion of inositol trisphosphate $\left(\mathrm{IP}_{3}\right)$ (and to a lesser extend of $\left.\mathrm{Ca}^{2+}\right), \mathrm{IP}_{3}$ receptors, DAG-activated nonselective cation channels, and $\mathrm{Ca}^{2+}$-activated chloride channels can contribute to synchronization, while largeconductance $\mathrm{Ca}^{2+}$-activated potassium channels have a desynchronizing effect.

Depending on the contractile state and agonist concentrations, different pathways become predominant, and can be revealed by carefully inhibiting the oscillatory component of their total activity. The phase shift between the $\mathrm{Ca}^{2+}$ and membrane potential oscillations can change, thus electrical coupling through gap junctions can mediate either synchronization or desynchronization. The effect of the endothelium is highly variable because it can simultaneously enhance the intercellular coupling and affect multiple SMC components. The model outlines a system of increased complexity and proposes potential synchronization mechanisms that need to be experimentally tested.

\subsection{Introduction}

Vessels from many vascular beds and species exhibit rhythmic oscillations in vessel diameter called vasomotion. These oscillations occur both in vitro and in vivo, independently from the heartbeat, respiration and sympathetic stimulation (Duling et al. 1981; Nilsson and Aalkjaer 2003). The ubiquity of vasomotion suggests an important role in the microcirculation, but further investigation is required to clarify its physiological 
relevance. Vasomotion results from coordinated contractile activity of many smooth muscle cells (SMCs), but the cellular mechanisms leading to the underlying SMC calcium $\left(\mathrm{Ca}^{2+}\right)$ oscillations and to synchronization are not fully understood. Intercellular coordination is of importance in other physiological systems, as well. Insights acquired from the investigation of vascular smooth muscle may reveal underlying mechanisms in other systems and particularly in tissues that share common characteristics with vascular SM (i.e. airway, gastrointestinal or lymphatic SM) (Berridge 2008; Imtiaz, von der Weid, and van Helden 2009).

In this study, we focus on vasomotion in rat small mesenteric arteries (RMA), which has been studied extensively at both experimental and theoretical levels. Differences in the underlying mechanisms may exist between vascular beds and even within a bed with vessels of different size. In RMA vasomotion, $\mathrm{Ca}^{2+}$ oscillates uniformly within individual SMCs, rather than in the form of intracellular waves (Peng et al. 2001; Mauban and Wier 2004). The regular whole-cell $\mathrm{Ca}^{2+}$ oscillations are generated spontaneously by the majority of SMCs, and only a small remaining fraction of SMCs exhibits $\mathrm{Ca}^{2+}$ oscillations as a result of the coupling with other cells. Under control conditions, the selfsustained regular $\mathrm{Ca}^{2+}$ oscillations originate from the internal stores with ryanodine (RyRs) or inositol trisphosphate $\left(\mathrm{IP}_{3} \mathrm{Rs}\right)$ receptors (Aalkjaer and Nilsson 2005; Haddock and Hill 2005).

The natural frequencies of $\mathrm{Ca}^{2+}$ oscillators (frequencies of isolated SMCs) are relatively steady under a given set of conditions but they differ between SMCs even within the same vascular segment due to the biological variability. The biological noise adds to the phase fluctuations between SMCs, and some cellular mechanisms may 
actively promote desynchronization. Therefore, sustained vasomotion requires synchronizing mechanisms that will override these desynchronizing effects and phase lock $\mathrm{Ca}^{2+}$ oscillations in a population of SMCs. Experimental studies have demonstrated that gap junction un-couplers abolish vasomotion (Matchkov et al. 2004; Aalkjaer and Nilsson 2005). Since gap junctions are nonselective channels permeable to ions and small molecules, they can mediate ionic coupling and transfer of signaling molecules. In general, a synchronizing signal can be very weak and produce no significant forced oscillations. It may only affect the phase rather than the amplitude of oscillations (weakly-coupled oscillators) (Pikovsky, Rosenblum, and Kurths 2001). Because a synchronizing signal can be superimposed on a larger sustained signal, its experimental inhibition is often difficult without affecting the whole system. For example, $\mathrm{Ca}^{2+}-$ activated chloride channels $\left(\mathrm{Cl}_{\mathrm{Ca}}\right)$ activity may be responsible for synchronization (Peng et al. 2001). However, it is the pulsatile increases in channel activity that cause synchronization and not the sustained current through this channel. $\mathrm{A} \mathrm{Cl}_{\mathrm{Ca}}$ channel blocker can inhibit the current and thus block the synchronizing signal but it may also affect membrane potential $\left(V_{\mathrm{m}}\right)$ levels and the intrinsic SMC oscillations. Thus, a more appropriate test will be to clamp the current to its average value, but such experimental intervention is not easy to implement. Furthermore, it is apparent that there can be several synchronizing mechanisms interacting in complex ways (Aalkjaer and Nilsson 2005). These and other factors make difficult to elucidate and control vasomotion experimentally. In this respect, computational models can provide a significant assistance to experimental studies. 
Based on a series of mathematical models of RMA vasomotion, Koenigsberger and coworkers concluded that synchronization is mediated by direct $\mathrm{Ca}^{2+}$ coupling between SMCs (Koenigsberger et al. 2004; Koenigsberger et al. 2005, 2006; Koenigsberger, Sauser, and Meister 2005). In the absence of $\mathrm{Ca}^{2+}$ coupling, identical SMCs were desynchronized by $\mathrm{Ca}^{2+}$-activated large conductance $\mathrm{K}^{+}$channels $\left(\mathrm{BK}_{\mathrm{Ca}}\right)$ and Gaussian noise in membrane conductances. Possible synchronizing effects of oscillatory $\mathrm{IP}_{3}$, generated by $\mathrm{Ca}^{2+}$-dependent phospholipase C (PLC)- $\delta$, and of stress-activated channels were suggested but predicted to be negligible. The controversial role of endothelial cells (ECs) and wall stress in vasomotion was ascribed mostly to their modulatory effect on the average SMC Ca ${ }^{2+}$ levels, leading to transitions into or out of the $\mathrm{Ca}^{2+}$ window for selfsustained $\mathrm{SMC} \mathrm{Ca}{ }^{2+}$ oscillations. In this scenario, the endothelium and wall stress do not affect the synchronization mechanism, and loss of vasomotion is caused by transition of SMCs from regular $\mathrm{Ca}^{2+}$ oscillations to irregular noise-induced $\mathrm{Ca}^{2+}$ flashes. On the other hand, Jacobsen et al. used theoretical modeling and experimental data to show that cGMP induces vasomotion in RMA through activation of cGMP-dependent $\mathrm{Cl}_{\mathrm{Ca}}$ channels (Jacobsen et al. 2007, 2007; Jacobsen et al. 2008). In a model with a heterogeneous population of SMCs, $\mathrm{Cl}_{\mathrm{Ca}}$ channels, activated by cGMP, depolarize $V_{\mathrm{m}}$ in phase with $\mathrm{Ca}^{2+}$ transients, and gap junctions transmit the depolarization to neighboring cells where voltage-operated $\mathrm{Ca}^{2+}$ channels (VOCC) coordinate $\mathrm{Ca}^{2+}$ oscillations. Increasing electrical coupling between SMCs enhanced synchronization, whereas intercellular $\mathrm{Ca}^{2+}$ diffusion was not important in this study. In this scheme, endothelium can promote synchronization through the nitric oxide (NO)-stimulated cGMP production and by reducing effective electrical resistance between SMCs. Other theoretical studies have 
focused on vasomotion in vessels other than RMA (Gonzalez-Fernandez and Ermentrout 1994; Parthimos, Edwards, and Griffith 1999, 2003; Parthimos et al. 2007). These studies are based on single cell models and examine the mechanism and modulation of oscillations but not their synchronization.

The theoretical studies by Koenigsberger et al. and Jacobsen et al. proposed novel descriptions of population dynamics in the vascular wall and provided valuable insights into possible synchronization mechanisms. These earlier studies can be further advanced by integrating potentially important components and pathways and improve quantitative descriptions. In (Koenigsberger et al. 2004; Koenigsberger et al. 2005, 2006; Koenigsberger, Sauser, and Meister 2005), for example, the models do not account for nonselective cation (NSC) and $\mathrm{Cl}_{\mathrm{Ca}}$ channels activity or cGMP effects, and significant intercellular permeabilities for $\mathrm{Ca}^{2+}$ are assumed. In (Jacobsen et al. 2007, 2007; Jacobsen et al. 2008), the models do not incorporate NSC channels, RyRs, $\mathrm{Ca}^{2+}$ dependent PLC activity, intercellular $\mathrm{IP}_{3}$ diffusion, or activation of $\mathrm{BK}_{\mathrm{Ca}}$ channels by cGMP. The effect of endothelium-derived factors or vasomotion in the absence of cGMP is not examined. Interestingly, $\mathrm{Ca}^{2+}$ transients are associated with $V_{\mathrm{m}}$ hyperpolarizations in the model of one group and with depolarizations in the model of the other group, and intercellular $\mathrm{Ca}^{2+}$ fluxes are significant in one study but not in the other. Thus, two fundamentally different models of synchronization in the same vascular bed have been proposed.

In this chapter, the previous hypotheses about the synchronization of self-sustained $\mathrm{Ca}^{2+}$ oscillators in RMA is tried to be reconciled and attempt is made to identify new candidate pathways for synchronization. Simulations suggest a complex and variable 
process that depends not only on vessel type but also on experimental conditions and stimuli. Several synchronizing/desynchronizing pathways appear to be involved simultaneously, and their relative magnitudes determine the initiation/termination of vasomotion.

\subsection{Methods}

We have previously developed detailed models of integrated $\mathrm{Ca}^{2+}$ and $V_{\mathrm{m}}$ dynamics in isolated EC and SMC, based primarily on data from RMA (Silva, Kapela, and Tsoukias 2007; Kapela, Bezerianos, and Tsoukias 2008). The isolated models were also combined into a two-cell (EC-SMC) and multicellular models to investigate myoendothelial communication and conducted vasoreactivity (Kapela, Bezerianos, and Tsoukias 2009; Kapela, Nagaraja, and Tsoukias 2010). To study the phenomenon of vasomotion, a multicellular model was implemented, similar to (Kapela, Nagaraja, and Tsoukias 2010). The individual SMCs and ECs are modeled as single compartments (lumped models) and SMCs can generate spontaneously self-sustained $\mathrm{Ca}^{2+}$ oscillations as a result of the nonlinear dynamics of the intracellular stores (Haddock and Hill 2005). Figure 5.1 shows a schematic diagram of the model. The reader is referred to our earlier publications for a more detailed description of the cellular models and cell coupling mechanisms (Silva, Kapela, and Tsoukias 2007; Kapela, Bezerianos, and Tsoukias 2008, 2009; Kapela, Nagaraja, and Tsoukias 2010). The model equations are summarized in the Appendix (Chapter 7) (Pg-190-205).

All cellular components and parameter values (including homocellular and heterocellular couplings) are the same as in our previous studies. The maximum SERCA rate, $\bar{I}_{\text {SERCA }}=20 \mathrm{pA}$, and the sarcoplasmic reticulum (SR) leak parameter, $R_{\text {leak }}=5.3 \times 10^{-}$ 
${ }^{5}$, allowed for oscillations generated at intermediate agonist stimulations by $\mathrm{Ca}^{2+}$-induced $\mathrm{Ca}^{2+}$ release via RyRs and slow refilling of SR (section 7.2.2 and Figure 5.5-A) (Kapela, Bezerianos, and Tsoukias 2008).

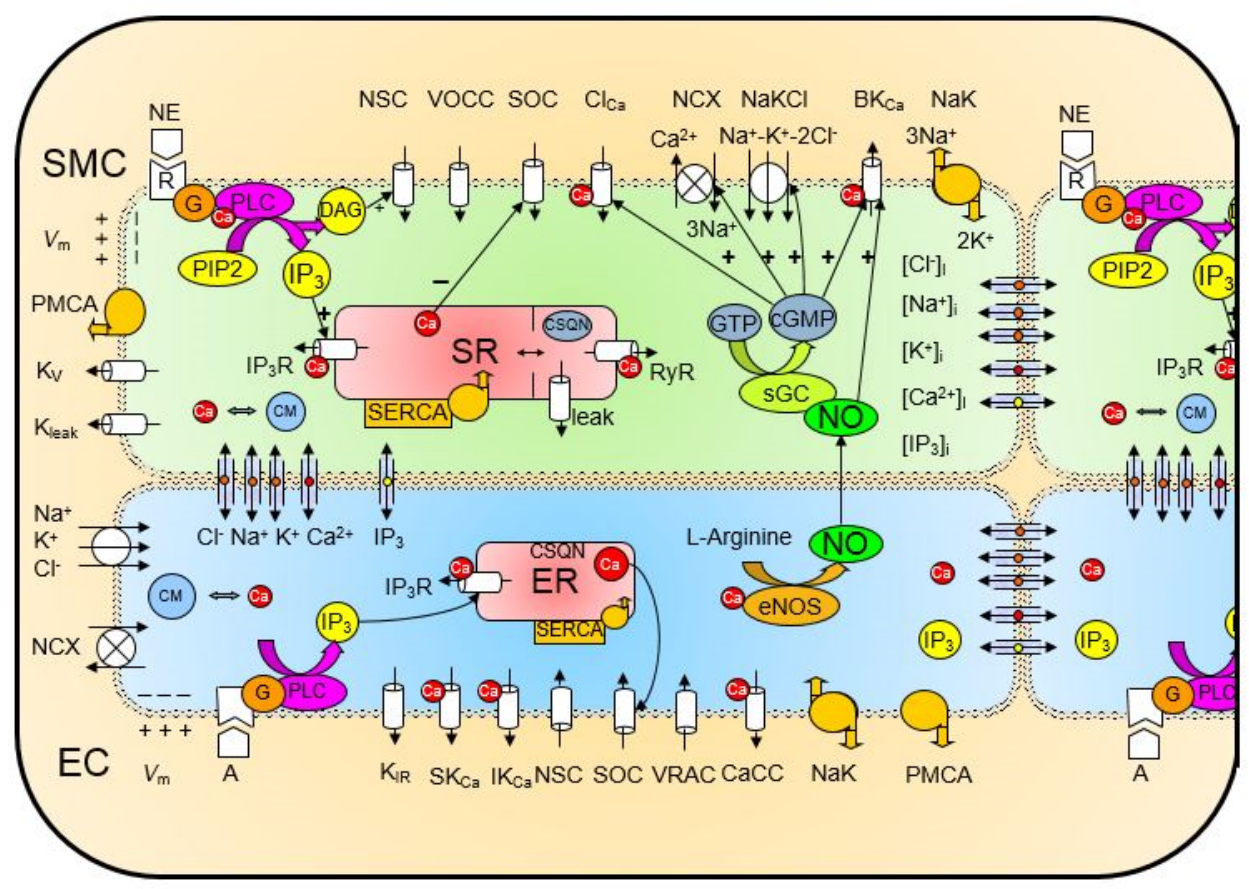

Figure 5.1 Schematic diagram of the ECs and SMCs coupled by nonselective homocellular and heterocellular gap junctions permeable to $\mathrm{Ca}^{2+}, \mathrm{Na}^{+}, \mathrm{K}^{+}$, and $\mathrm{Cl}^{-}$ions, and $\mathrm{IP}_{3} . \mathrm{K}_{\text {ir }}$ - inward rectifier $\mathrm{K}^{+}$ channel; VRAC - volume-regulated anion channel; $\mathrm{SK}_{\mathrm{Ca}}, \mathrm{IK}_{\mathrm{Ca}}$ and $\mathrm{BK}_{\mathrm{Ca}}$ - small-, intermediate-, and largeconductance $\mathrm{Ca}^{2+}$-activated $\mathrm{K}^{+}$channels; SOC - store-operated channel; NSC - nonselective cation channel, $\mathrm{CaCC}$ and $\mathrm{Cl}_{\mathrm{Ca}}-\mathrm{Ca}^{2+}$-activated chloride channel; $\mathrm{NaK}-\mathrm{Na}^{+}-\mathrm{K}^{+}$-ATPase; PMCA - plasma membrane $\mathrm{Ca}^{2+}$-ATPase; $\mathrm{NCX}-\mathrm{Na}^{+} / \mathrm{Ca}^{2+}$ exchanger; $\mathrm{NaKCl}-\mathrm{Na}^{+}-\mathrm{K}^{+}-\mathrm{Cl}^{-}$cotransport; $\mathrm{K}_{\mathrm{v}}-$ voltagedependent $\mathrm{K}^{+}$channel; $\mathrm{K}_{\text {leak }}$ - unspecified $\mathrm{K}^{+}$leak current; VOCC - voltage-operated $\mathrm{Ca}^{2+}$ channels; $\mathrm{SR} / \mathrm{ER}$ - sarco/endoplasmic reticulum; $\mathrm{IP}_{3} \mathrm{R}-\mathrm{IP}_{3}$ receptor; RyR - ryanodine receptor; SERCA - SR/ER $\mathrm{Ca}^{2+}$-ATPase; CSQN - calsequestrin; CM - calmodulin; R - receptor; G - G-protein; DAG diacylglycerol; PLC - phospholipase C; sGC - soluble guanylate cyclase; cGMP - cyclic guanosine monophosphate.

$\mathrm{IP}_{3} \mathrm{Rs}$ are neither necessary nor sufficient for self-sustained oscillations in our model.

To account for the natural variability between SMCs, selected cell parameters (e.g., $R_{\text {leak }}$

or $\bar{I}_{\text {SERCA }}$ ) were multiplied by the perturbation coefficient $\delta$ : 


$$
\delta(1: N)=\delta_{\max }([\operatorname{rand}(1: \mathrm{N})-\min (\operatorname{rand}(1: \mathrm{N}))] /[\max (\operatorname{rand}(1: \mathrm{N}))-\min (\operatorname{rand}(1: \mathrm{N}))])
$$

\section{Equation 5.1}

where $N$ is the total number of SMCs, $\delta_{\max }-$ maximum perturbation (control $\delta_{\max }=1.2$ ), rand $(1: N)$ - vector of pseudo-random values drawn from the unit interval. The resulting oscillations in individual SMCs have natural frequencies within a predetermined physiological range (Figure 5.5-B) (Jacobsen et al. 2007; Jacobsen et al. 2008).

Simulations were repeated with different sets of perturbations and the average behavior of the model was considered. Random variations simulating time-dependent biological noise were also implemented similar to (Koenigsberger et al. 2004). Simulations with either intercellular variability or time-dependent noise show similar desynchronizing effects. Thus, only simulations with intercellular variations are presented. Models with different number of cells (2-100 SMCs and ECs), SMC layers (15), and arrangement (serial or cylindrical) were implemented and displayed similar synchronization properties. To reduce computational time, the control model is composed of five SMCs connected in series and overlapping five identical ECs arranged perpendicular to the SMCs, so that each SMC connects to five ECs.

Endothelial gap junctions are more prevalent than smooth muscle and myoendothelial gap junctions. This translates into a low EC-EC resistance $\left(R_{\mathrm{gj}}^{\mathrm{EC}-\mathrm{EC}}=3.3 \mathrm{M} \Omega\right)$, an intermediate SMC-SMC resistance $\left(R_{\mathrm{gj}}^{\mathrm{SMC}-\mathrm{SMC}}=87.4 \mathrm{M} \Omega\right)$, and a high EC-SMC resistance per SMC $\left(R_{\mathrm{gj}}^{\mathrm{EC}-\mathrm{SMC}}=900 \mathrm{M} \Omega\right)($ Kapela, Nagaraja, and Tsoukias 2010). The permeability of the gap junctions to $\mathrm{K}^{+}, \mathrm{Cl}^{-}, \mathrm{Na}^{+}$and $\mathrm{Ca}^{2+}$ ions was calculated according to section 7.1.1 from the corresponding intercellular resistance, as described previously 
(Kapela, Bezerianos, and Tsoukias 2009). This yields $P_{\mathrm{S}}^{\mathrm{EC}-\mathrm{EC}}=0.384 \mathrm{pL} / \mathrm{s}, P_{\mathrm{S}}^{\mathrm{SMC}-\mathrm{SMC}}=$ $0.0145 \mathrm{pL} / \mathrm{s}$, and $P_{\mathrm{S}}^{\mathrm{EC}-\mathrm{SMC}}=0.0014 \mathrm{pL} / \mathrm{s}$, where $\mathrm{S}=\mathrm{K}^{+}, \mathrm{Cl}^{-}, \mathrm{Na}^{+}$and $\mathrm{Ca}^{2+}$. $\mathrm{A}$ myoendothelial $\mathrm{IP}_{3}$ permeability of $0.05 \mathrm{~s}^{-1}$ (i.e., $P_{\mathrm{IP} 3}^{\mathrm{EC}-\mathrm{SMC}}=0.05 \mathrm{pL} / \mathrm{s}$ ) per cell was assumed in agreement with (Koenigsberger et al. 2005), and the corresponding SMC-to$\mathrm{SMC} \mathrm{IP}_{3}$ permeability was adjusted based on the gap junction resistance ratio to 0.53 $\mathrm{pL} / \mathrm{s}$. This value has not been experimentally derived and represents a rough approximation. Furthermore $\mathrm{IP}_{3}$ flux is assumed proportional to the chemical and not the electrochemical gradient between two cells (section 7.1.2). The uncertainty in $\mathrm{IP}_{3}$ permeability, particularly, can have a significant impact on model predictions and thus a wide range of values was examined.

To better monitor transitions in and out of synchronization, a mean correlation coefficient of $\mathrm{Ca}^{2+}$ oscillations was calculated from all SMC-SMC combinations at each time step within $300 \mathrm{~s}$ time window:

$$
\overline{\operatorname{corr}}(t)=\frac{2}{N(N-1)} \sum_{n>m} \operatorname{corr}\left(\mathrm{Ca}^{n}(t: t+w), \mathrm{Ca}^{m}(t: t+w)\right) \quad n, m=1 \ldots N
$$

\section{Equation 5.2}

where $t$ - simulation time, $w=100 \mathrm{~s}-$ time window, $\mathrm{Ca}^{n}(t: t+w)-\mathrm{Ca}^{2+}$ signal from time $t$ to $t+w$ in $n$-th SMC, corr - the correlation coefficient. For a single SMC-SMC pair, the correlation coefficient ranges from -1 to 1 , where $-1,0$, and 1 correspond to complete anti-phase, uncorrelated, and in-phase oscillations, respectively. For a large population, the mean correlation varies from 0 to 1 . The cells are considered synchronized when the mean correlation is close to 1 and remains stable over a significant number of oscillation periods. 
The system of ordinary differential equations describing the model (11 ODEs per EC and 26 ODEs per SMC) was solved numerically in FORTRAN 90 using the Gear's backward differential formula method for stiff systems with maximum time step of $4 \mathrm{~ms}$. Long simulated times were used (up to $80 \mathrm{~min}$ ) to eliminate transient and resetting effects (Figure 5.5-D), and thus, considerable computation times were required for models with many cells. The computations were significantly slower in the presence of time dependent noise.

\subsection{Results}

\subsection{1 $\mathrm{IP}_{3}$ and $\mathrm{Ca}^{2+}$ Coupling}

In the presence of the endothelial layer, synchronized and unsynchronized oscillations in the SMCs were observed, depending on the level of smooth muscle and endothelial stimulation. For example, $0.8 \mu \mathrm{M}$ norepinephrine (NE) and 1 a.u. acetylcholine (Ach) gave rise to synchronized $\mathrm{Ca}^{2+}$ oscillations (Figure 5.2-A and Figure 5.5-A). Under the specific conditions in Figure 5.2-A, synchronization was lost by blocking $\mathrm{IP}_{3}$ diffusion between SMCs $\left(P_{\mathrm{IP} 3}^{\mathrm{SMC}-\mathrm{SMC}}=0\right.$ in section 7.1.2). Similar behavior was observed in a larger ensemble of SMCs and ECs (Figure 5.7). In the presence of $\mathrm{IP}_{3}$ diffusion removal of electrical coupling $\left(P_{\mathrm{S}}^{\mathrm{SMC}-\mathrm{SMC}}=0 ; \mathrm{S}=\mathrm{Ca}^{2+}, \mathrm{Na}^{+}, \mathrm{K}^{+}, \mathrm{Cl}^{-}\right.$in section 7.1.1) had no effect (data not shown). However, when both the ionic and $\mathrm{IP}_{3}$ couplings are present, clamping NSC channels to the mean value of their DAG-induced fluctuating activity (section 7.2.1.5) causes loss in synchronization (DAG clamp; Figure 5.2-B) (see also NSC channel section below). 

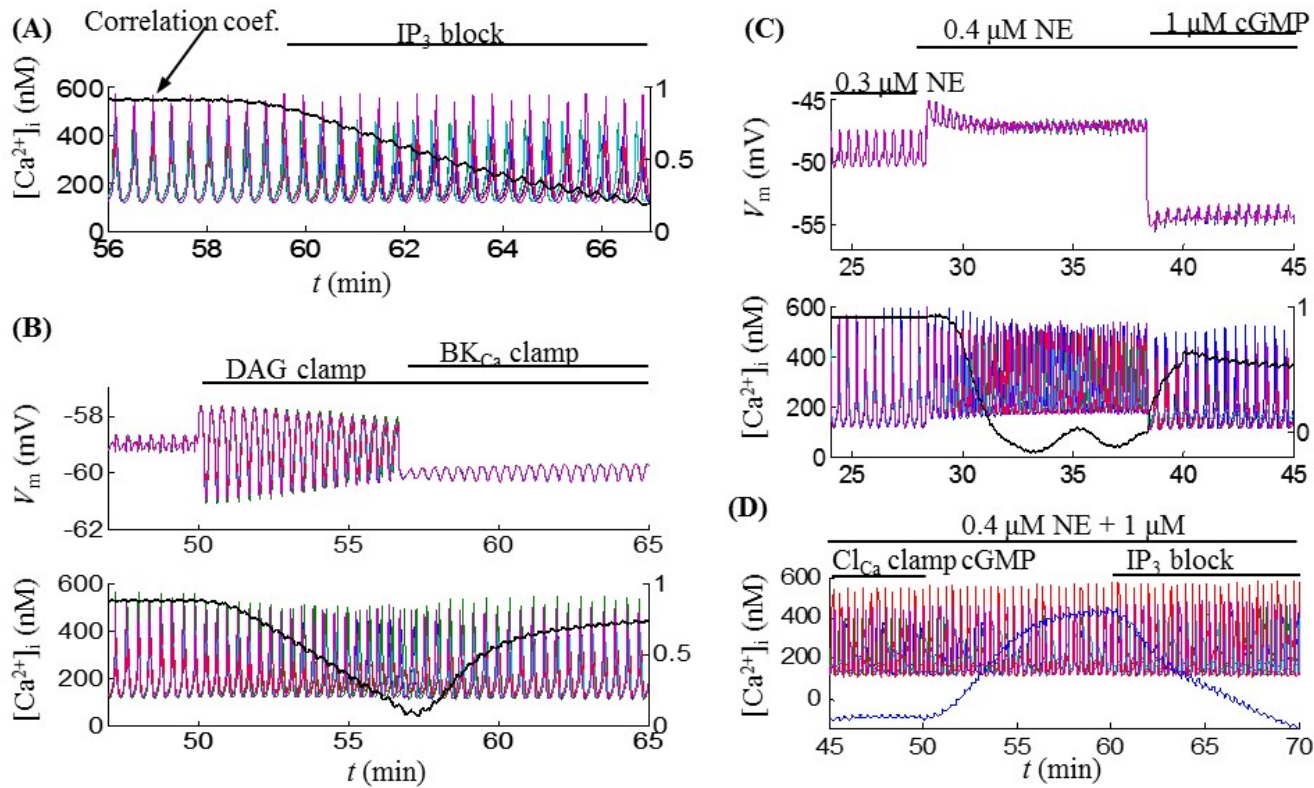

Figure 5.2 (A) A population of five SMCs and five ECs is stimulated by NE $(0.8 \mu \mathrm{M})$ and Ach ( 1 a.u. $)$. $\mathrm{Ca}^{2+}$ oscillations in the SMCs are shown. Inhibition of intercellular $\mathrm{IP}_{3}$ diffusion desynchronized $\mathrm{SMC} \mathrm{Ca}{ }^{2+}$ oscillations. A correlation coefficient (solid line) quantifies level of synchronization with total synchronization having a value of 1 . (B) Same conditions as in (A). $V_{\mathrm{m}}(T o p)$ and $\mathrm{Ca}^{2+}$ oscillations in SMCs (Bottom) are shown. Clamping DAG concentration to its mean value leads to desynchronization and demonstrates the importance of NSC channels. The synchronization was restored by clamping $\mathrm{BK}_{\mathrm{Ca}}$ activation with mean cytosolic $\mathrm{Ca}^{2+}$. (C) In a population of five SMCs (ECs absent), NE $(0.3 \mu \mathrm{M})$ induced synchronized $V_{\mathrm{m}}$ (Top) and $\mathrm{Ca}^{2+}$ oscillations (Bottom). Increasing $\mathrm{NE}$ concentration $\left(\begin{array}{lll}0.4 \mu \mathrm{M}) & \mathrm{C}\end{array}\right.$ desynchronized SMCs, while exogeneous cGMP hyperpolarizes and resynchronized $\mathrm{Ca}^{2+}$ oscillations through its action on $\mathrm{Cl}_{\mathrm{Ca}}$ channels. (D) Same conditions as in $(\mathrm{C})$. $\mathrm{Cl}_{\mathrm{Ca}}$ channels are clamped with mean cytosolic $\mathrm{Ca}^{2+}$. Removal of clamping restores synchronization which is lost after blocking intercellular $\mathrm{IP}_{3}$ diffusion between SMCs.

Hence, the $\mathrm{IP}_{3}$ coupling was a necessary but not the only factor contributing to synchronization under these conditions. The $\mathrm{IP}_{3}$-mediated synchronization occurs through the following series of events: $\mathrm{Ca}^{2+}$ oscillations in one cell generate $\mathrm{IP}_{3}$ oscillations in the cell -as a result of the $\mathrm{Ca}^{2+}$ dependence of the PLC activity $\left(r_{\mathrm{h}, \mathrm{G}}\right.$ in section 7.2.3) - and by diffusion through gap junctions in neighboring cells. This modulates the $\mathrm{IP}_{3} \mathrm{Rs}$ (section 7.2.2), cytosolic $\mathrm{Ca}^{2+}$ levels and accelerates or delays CICR through RyR in the neighboring cell (i.e., $\mathrm{Ca}^{2+} \rightarrow \mathrm{PLC} \rightarrow \mathrm{IP}_{3} \rightarrow \mathrm{GJ} \rightarrow \mathrm{IP}_{3} \mathrm{R} \rightarrow \mathrm{Ca}^{2+}$ ). Through a parametric analysis we determined the minimum value of $\mathrm{IP}_{3}$ permeability ( 
$P_{\mathrm{IP} 3}^{\mathrm{SMC}-\mathrm{SMC}}=0.058 \mathrm{pL} / \mathrm{s}$ ) for intercellular $\mathrm{IP}_{3}$ diffusion to be able to synchronize the cells under these conditions. In the absence of ECs, strong $\mathrm{IP}_{3}$-mediated synchronization was possible if the permeability of gap junctions to $\mathrm{IP}_{3}$ was increased three fold from its control value (i.e., $\left.P_{\mathrm{IP} 3}^{\mathrm{SMC}-\mathrm{SMC}}=1.59 \mathrm{pL} / \mathrm{s}\right)\left(\right.$ Results not shown). Direct $\mathrm{Ca}^{2+}$ diffusion between SMCs did not play a significant role. However, $\mathrm{Ca}^{2+}$ coupling alone could provide good synchronization if $\mathrm{Ca}^{2+}$ permeability was increased a hundred-fold from its control value $\left(P_{\mathrm{Ca}}^{\mathrm{SMC}-\mathrm{SMC}}=1.45 \mathrm{pL} / \mathrm{s}\right)$. A change in the gap junctional resistance from 84 $\mathrm{M} \Omega$ to less than $1 \mathrm{M} \Omega$ between SMCs is needed to justify such value.

\subsubsection{Electrical Coupling}

Electrical coupling can have a synchronizing or desynchronizing effect. The net effect of intercellular current flux on synchronization depends on a number of parameters such as the level of agonist stimulation and can be altered by channel inhibition/activation. This model behavior is attributed to the $\mathrm{Ca}^{2+}$ dependency of three channels, the $\mathrm{Cl}_{\mathrm{Ca}}$, the NSC, and the $\mathrm{BK}_{\mathrm{Ca}}$. Current spread through gap junctions will synchronize/desynchronize depending on the balance between the synchronizing potential of these three currents. [Note: not simply the balance of magnitude of these currents].

\subsubsection{NSC Channels}

Due to the $\mathrm{Ca}^{2+}$ dependency of the PLC pathway (section 7.2.3), $\mathrm{Ca}^{2+}$ oscillations generate concomitant oscillations in DAG concentration. In simulations with or without ECs, elimination of DAG oscillations by clamping DAG to its average concentration can yield loss of synchronization. For example, clamping DAG terminated synchronization in 
a population of SMCs and ECs stimulated by $0.8 \mu \mathrm{M}$ NE and 1 a.u. Ach (Figure 5.2-B). The termination was consistent in simulations $(n=10)$ with sets of SMCs of different frequency distributions. The NSC clamping also desynchronized SMCs under the conditions of Figure 5.2-C and 5.3-A, but not of Figure 5.3-C. The coordination is restored by concurrent clamping of $\mathrm{BK}_{\mathrm{Ca}}$ channels to their activity at mean $\mathrm{Ca}^{2+}$ levels. The results suggest that NSC channels contribute to synchronization by counteracting $\mathrm{BK}_{\mathrm{Ca}}$ channels. The NSC-mediated synchronization involves oscillatory DAG, generated by the $\mathrm{Ca}^{2+}$ dependency of PLC, which in turn modulates NSC channel activation. Every time that $\mathrm{Ca}^{2+}$ increases in one cell, NSC activates, $V_{\mathrm{m}}$ depolarizes, and this activates, through electrical coupling, VOCC in neighboring cells to increase their cytosolic $\mathrm{Ca}^{2+}$ (i.e., $\mathrm{Ca}^{2+} \rightarrow \mathrm{PLC} \rightarrow \mathrm{DAG} \rightarrow \mathrm{NSC} \rightarrow \mathrm{GJ} \rightarrow \mathrm{VOCC} \rightarrow \mathrm{Ca}^{2+}$ ).

\subsubsection{2 $\mathrm{Cl}_{\mathrm{Ca}}$ Channels}

Figure 5.2-C depicts $\mathrm{Ca}^{2+}$ oscillations in a population of SMCs, in the absence of ECs, at two different NE concentrations and after addition of cGMP. Synchronized oscillations appear at $0.3 \mu \mathrm{M}$ NE and unsynchronized at $0.4 \mu \mathrm{M}$ NE. The higher NE produces stronger depolarization of SMCs mainly through the NSC channel but causes desynchronization through the activity of $\mathrm{BK}_{\mathrm{Ca}}$ channels (see $\mathrm{BK}_{\mathrm{Ca}}$ section below). Addition of cGMP in the absence of ECs activates depolarizing $\mathrm{Cl}_{\mathrm{Ca}}$ channels but also hyperpolarizing $\mathrm{BK}_{\mathrm{Ca}}$. In simulations presented in Figure 5.2-C, $1 \mu \mathrm{M}$ cGMP repolarized SMCs by increasing $\mathrm{BK}_{\mathrm{Ca}}$ conductance more than $\mathrm{Cl}_{\mathrm{Ca}}$ conductance. This is consistent with the relaxing effect of cGMP and experimental data showing a two-fold increase in whole cell conductance after cGMP administration to isolated SMC (probably due to activation of $\mathrm{K}^{+}$channels) (Rahman et al. 2005). cGMP, however, synchronized $\mathrm{Ca}^{2+}$ 
oscillations through the activity of $\mathrm{Cl}_{\mathrm{Ca}}$ channels (Figure 5.2-C). Thus, the channel responsible for transition in/out of synchronization is not necessarily the same as the channel mostly affecting mean membrane potential.

The importance of $\mathrm{Cl}_{\mathrm{Ca}}$ channels was confirmed by loss of coordination after clamping $\mathrm{Cl}_{\mathrm{Ca}}$ activity to its value at mean cytosolic $\mathrm{Ca}^{2+}$ levels during the oscillations (Figure 5.2D). cGMP-induced synchronization was lost after blocking the SMC-SMC IP 3 coupling under the conditions of Figure 5.2-D. If the cGMP dependence of $\mathrm{BK}_{\mathrm{Ca}}$ was removed, 1 $\mu \mathrm{M}$ cGMP depolarized (rather than hyperpolarized) $V_{\mathrm{m}}$ and did not synchronize the cells (data not shown). However, the synchronization was restored if cGMP was accompanied with repolarization of SMCs by 1.3 -fold increase of the maximum NaK pump rate (section 7.2.1.10), similarly to (Jacobsen et al. 2007) (data not shown). In this case, electrical coupling alone was sufficient for synchronization, indicating that the depolarizing $\mathrm{Cl}_{\mathrm{Ca}}$ and $\mathrm{NSC}$ channels were dominant over $\mathrm{BK}_{\mathrm{Ca}}$ channels. Thus, $\mathrm{Cl}_{\mathrm{Ca}}$ channels were necessary but not always sufficient for cGMP-dependent synchronization and the ability of cGMP to synchronize a population of SMCs may depend on $V_{\mathrm{m}}$.

The ability of exogenous cGMP to synchronize EC denuded vessels may not be always indicative for the role of $\mathrm{Cl}_{\mathrm{Ca}}$ in intact vessel synchronization. In the Supplement (section 5.7), we show representative simulations after $\mathrm{Cl}_{\mathrm{Ca}}$ clamping or blocking, when the EC layer is present. For moderate levels of Ach and NE stimulation (i.e. Ach=1 au; $\mathrm{NE}=0.8 \mathrm{uM}$ ), clamping $\mathrm{Cl}_{\mathrm{Ca}}$ did not elicit desynchronization (Figure 5.6-A). Blocking $\mathrm{Cl}_{\mathrm{Ca}}$ under the same conditions was effective in terminating synchronization in many simulations with different sets of SMCs (Figure 5.6-B). Terminations of synchronization 
with $\mathrm{Cl}_{\mathrm{Ca}}$ block when $\mathrm{Cl}_{\mathrm{Ca}}$ clamp has no effect, is attributed to changes in $V_{\mathrm{m}}$ and suggests that synchronization was not due to $\mathrm{Cl}_{\mathrm{Ca}}$ current under this particular conditions.

\subsubsection{BK $K_{\text {Ca }}$ channels}

$\mathrm{BK}_{\mathrm{Ca}}$ channels generate hyperpolarizing current in response to $\mathrm{Ca}^{2+}$ elevation, and thus have a desynchronizing effect. The importance of this effect depends on model configuration and experimental conditions. In Figure 5.2-C (absence of ECs) higher concentration of NE produces stronger depolarization of SMCs by further activation of NSC channels, but also sensitizes $V_{\mathrm{m}}$-dependent $\mathrm{BK}_{\mathrm{Ca}}$ channels to $\mathrm{Ca}^{2+}$. The sensitivity of $\mathrm{BK}_{\mathrm{Ca}}$ conductance to $\mathrm{Ca}^{2+}$ becomes more significant than the sensitivity of $\mathrm{NSC}$ and $\mathrm{Cl}_{\mathrm{Ca}}$ to $\mathrm{Ca}^{2+}$. As a result $V_{\mathrm{m}}$ repolarizes (instead of depolarizing) with $\mathrm{Ca}^{2+}$ transients and synchronization is lost (Figure 5.2-C). Application of cGMP counteracts $\mathrm{BK}_{\mathrm{Ca}}$ activity by increasing the synchronizing effect of $\mathrm{Cl}_{\mathrm{Ca}}$ (Figure 5.2-C). The significant desynchronizing role of the $\mathrm{BK}_{\mathrm{Ca}}$ predicted by the model depends on the assumed $\mathrm{Ca}^{2+}$ sensitivity of the $\mathrm{BK}_{\mathrm{Ca}}$ current (Benham et al. 1986), and in some tissues $\mathrm{BK}_{\mathrm{Ca}}$ activity may be less sensitive to $\mathrm{Ca}^{2+}$ than assumed in this study.

\subsubsection{Role of EDHF}

Figure 5.3-A.investigates the effect of endothelium-derived hyperpolarizing factor (EDHF) on synchronization. The SM layer was prestimulated with NE and the ECs were stimulated with Ach at appropriate levels to induce synchronized oscillations. Blockade of the $\mathrm{SK}_{\mathrm{Ca}}$ and $\mathrm{IK}_{\mathrm{Ca}}$ channels in the ECs (section 7.3.1.2) (i.e., blockade of EDHF response) has a desynchronizing effect under these conditions. This is attributed to the resulting depolarization and an increased role of $\mathrm{BK}_{\mathrm{Ca}}$ channels as described above. Thus, simulations show that EDHF can affect cell synchronization although the effect is 
indirect, through the modulation of $V_{\mathrm{m}}$ and the alteration of $\mathrm{BK}_{\mathrm{Ca}}$ channel contribution.

Experimental studies in RMA have demonstrated that the inhibition of $\mathrm{SK}_{\mathrm{Ca}}$ and $\mathrm{IK}_{\mathrm{Ca}}$ channels terminated vasomotion by desynchronizing $\mathrm{Ca}^{2+}$ oscillations (Mauban and Wier 2004). However, Seppey et al. suggested that the EDHF effect is only to induce transition from more contracted state to less contracted state, and depending on NE concentration this may induce or stop vasomotion (Seppey et al. 2008).
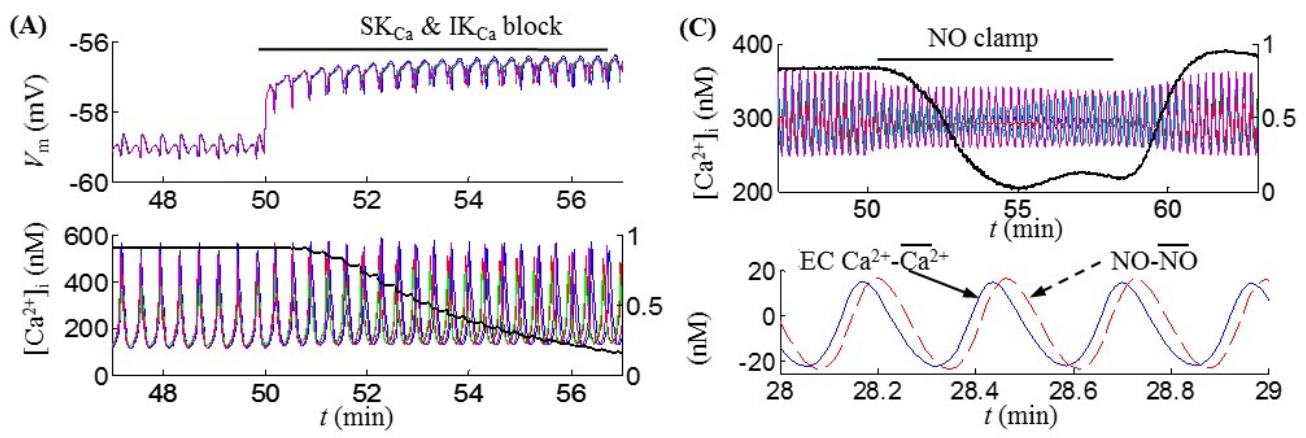

(B)
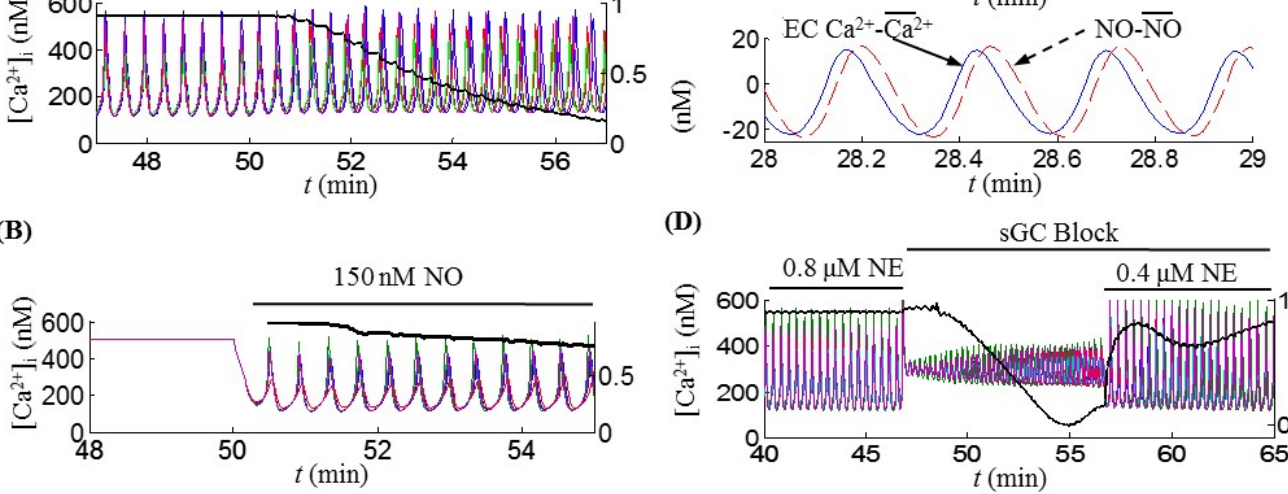

(D)

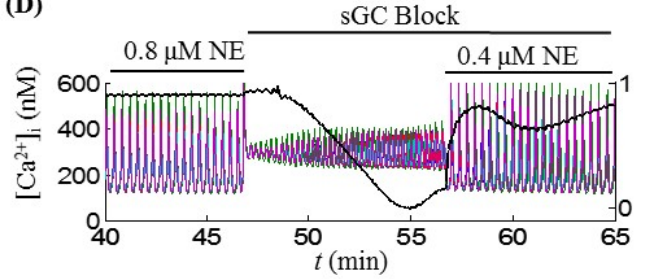

Figure 5.3 (A) Model simulations in five SMCs and five ECs stimulated with NE $(1 \mu \mathrm{M})$ and Ach $(0.7$ a.u.). Inhibition of endothelial $\mathrm{SK}_{\mathrm{Ca}}$ and $\mathrm{IK}_{\mathrm{Ca}}$ channels depolarizes $V_{\mathrm{m}}(T o p)$ and desynchronizes $\mathrm{Ca}^{2+}$ oscillations (Bottom). (B) In a population of five SMCs prestimulated with NE $(1 \mu \mathrm{M})$ (ECs absent), exogenous NO $(150 \mathrm{nM})$ reduces mean $\mathrm{SM} \mathrm{Ca}^{2+}$ from non-oscillatory to oscillatory domain and this yields synchronized oscillations. (C) Top: Five ECs and five SMCs are stimulated with Ach (0.6 a.u.) and NE (1.8 $\mu \mathrm{M}) . \mathrm{Ca}^{2+}$ oscillations in SMCs are shown. Clamping the endothelium-derived NO to its average concentration reduced $\mathrm{Ca}^{2+}$ oscillations and abolished synchronization. Bottom: Oscillatory components of $\mathrm{EC} \mathrm{Ca}^{2+}$ (solid line) and endothelium-derived NO (dashed line) show phase shift. (D) In the presence of Ach (1 a.u.) and endothelial NO release, inhibition of cGMP production by block of SM sGC reduced amplitude and synchronization of $\mathrm{Ca}^{2+}$ oscillations through depolarization and $\mathrm{BK}_{\mathrm{Ca}}$ channels. The effect of sGC block could be compensated by NE adjustment from $0.8 \mu \mathrm{M}$ to $0.4 \mu \mathrm{M}$.

\subsubsection{Role of NO}

The net result of $\mathrm{NO}$ on synchronization is difficult to predict due to multiple targets of $\mathrm{NO} / \mathrm{cGMP}$ pathway and their differential effects on $\mathrm{Ca}^{2+}$ dynamics. In simulations, addition of exogenous NO $(0.15-0.4 \mu \mathrm{M})$ in the presence of $\mathrm{NE}(0.8-1.2 \mu \mathrm{M})$ repolarized 
SMCs to their oscillatory domain and gave rise to synchronized oscillations (Figure 5.3-B and Figure 5.5-A). This is in agreement with experimental data showing vasomotion induced by NO in endothelium denuded RMA (Seppey et al. 2008). Some combinations of $\mathrm{NO}$ and $\mathrm{NE}$ induced uncorrelated oscillations, predicting a concentration-dependent NO effect.

Endogenous, endothelium-derived NO (section 7.1.3) (i.e., endothelium-derived relaxing factor (EDRF)) may have different effect on vasomotion than similar levels of exogenous NO. EDRF can oscillate with $\mathrm{SM} \mathrm{Ca}^{2+}$ as a result of myoendothelial communication, and can affect synchronization dynamics in parallel to EDHF. Indeed, at certain combinations of NE and Ach (for example 1.8 $\mu \mathrm{M} \mathrm{NE}$ and 0.6 a.u. Ach), clamping NO to its time-averaged concentration terminated synchronization (Figure 5.3C). Diffusion of $\mathrm{IP}_{3}$ from SMCs to ECs through myoendothelial gap junctions generated $\mathrm{EC} \mathrm{Ca}^{2+}$ and NO oscillations (Figure 5.3-C). Corresponding $\mathrm{Ca}^{2+}$ and NO oscillations in SMC may have a significant phase-shift due to delays in $\mathrm{IP}_{3}$ diffusion, $\mathrm{NO}$ production, and $\mathrm{NO}$ diffusion. The out-of-phase $\mathrm{NO}$ acting on $\mathrm{BK}_{\mathrm{Ca}}$ can then inhibit the desynchronizing effect of $\mathrm{BK}_{\mathrm{Ca}}$.

At some concentrations of $\mathrm{NE}$ and Ach, synchronized $\mathrm{Ca}^{2+}$ oscillations were possible with complete EDRF inhibition (data not shown). Thus, the model predicts possibility of vasomotion with EDRF present or blocked. Similarly in RMA, inhibition of NO by LNAME did not prevent $\mathrm{Ca}^{2+}$ synchronization (Sell, Boldt, and Markwardt 2002), although it reduced significantly the amplitude of vasomotion (Mauban and Wier 2004; Rahman et al. 2005). Total inhibition of EDRF (i.e., inhibition of NO production) is different than blockade of sGC/cGMP pathway since $\mathrm{NO}$ can also activate $\mathrm{BK}_{\mathrm{Ca}}$ directly. 
Inhibition of sGC/cGMP pathway in the model $\left(V_{\mathrm{cGMP}, \max }=0\right.$ in section 7.2.4) reduced the amplitude and synchronization of $\mathrm{Ca}^{2+}$ oscillations, although adjustment of $\mathrm{NE}$ concentration could compensate for the changes and restored the coordination (Figure 5.3-D). In RMA, ODQ (sGC inhibitor) did not eliminate vasomotion but had modulatory role (Mauban and Wier 2004).

\subsection{Discussion}

The study shows how synchronization in a population of SMCs can be achieved/lost as a result of the competition between synchronizing and desynchronizing factors. Previously recognized pathways are evaluated in a more complex cellular model system and under different stimulatory conditions. The study also suggests two alternative pathways for synchronization. Intercellular $\mathrm{IP}_{3}$ diffusion and current flux generated through NSC channels have the potential to affect synchronization. Thus, there can be several synchronizing mechanisms that work individually, in synergy or redundancy, depending on stimulatory conditions. The model simulations depict a system of increased complexity, where the relative contribution of different pathways is very sensitive to experimental conditions.

The synchronizing pathways share certain common features and can be grouped into diffusion-mediated and electrically-mediated (Figure 5.4). Synchronization mediated by small species requires: a) $\mathrm{Ca}^{2+}$-induced oscillations in the concentration of the mediator, if the mediator is not $\mathrm{Ca}^{2+}, \mathrm{b}$ ) sufficient intercellular flux and c) modulation by the mediator of a component participating in the oscillatory cycle in the neighboring cell. Diffusion of the mediator over long distances or the ability to generate propagating waves is not required. 


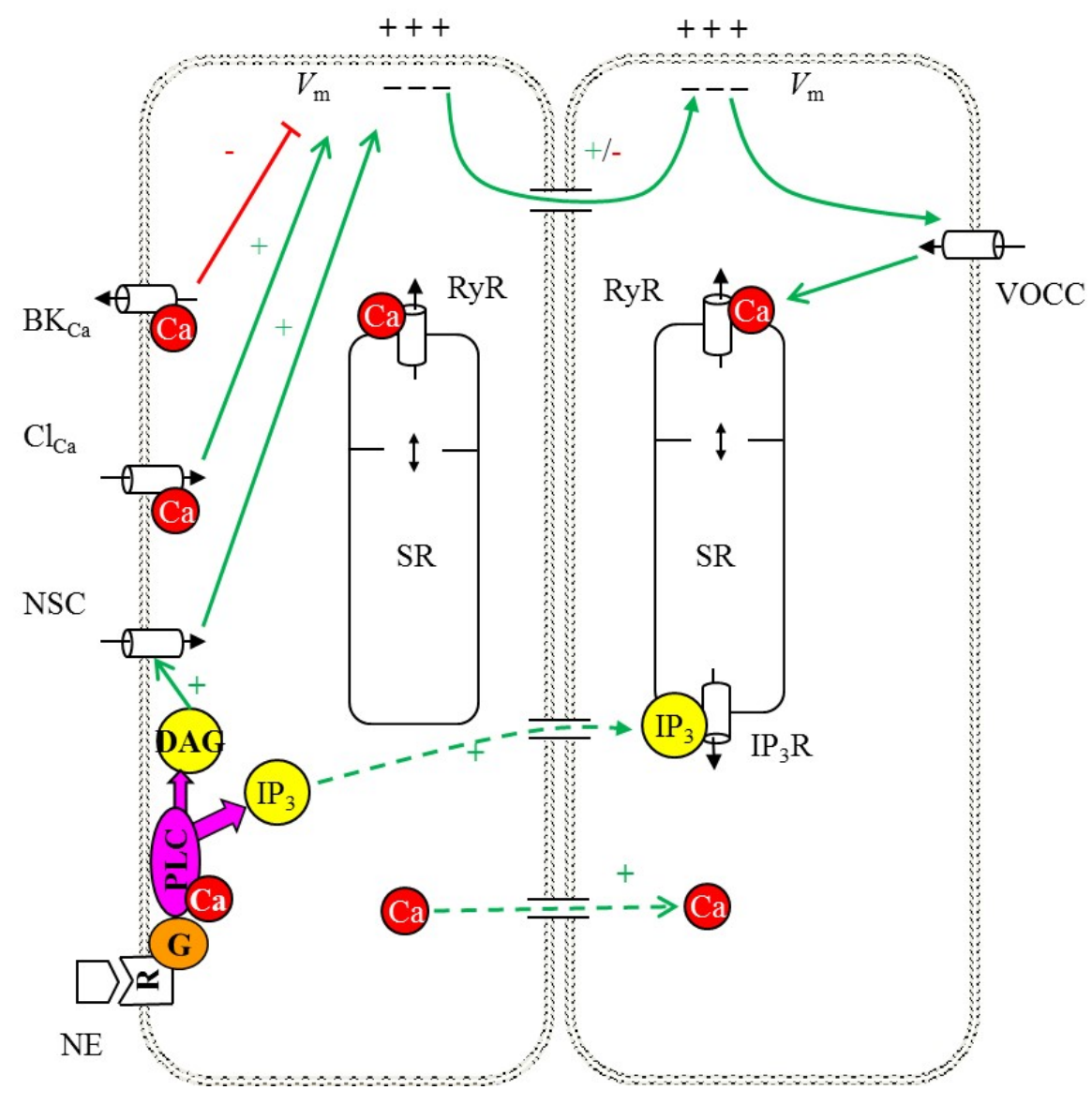

Figure 5.4 Schematic diagram of signaling pathways that may be involved in $\mathrm{Ca}^{2+}$ synchronization, as predicted by the model. $\mathrm{Ca}^{2+}$ increase in one SMC (left) can activate directly $\mathrm{Cl}_{\mathrm{Ca}}$ and $\mathrm{BK}_{\mathrm{Ca}}$ channels, and indirectly NSC channels through $\mathrm{Ca}^{2+}$-sensitive PLC. Variations in channels' activity generate $V_{\mathrm{m}}$ oscillations, which spread through gap junctions to neighboring SMCs (right) and modulate VOCC channels and RyRs. Oscillatory $\mathrm{IP}_{3}$, generated by $\mathrm{Ca}^{2+}$-dependent PLC, can diffuse to other cells, and may also have a synchronizing effect by acting on $\mathrm{IP}_{3}$ receptors. $\mathrm{Ca}^{2+}$ elevation in one cell may increase $\mathrm{Ca}^{2+}$ in neighboring cells by direct diffusion of $\mathrm{Ca}^{2+}$ through the gap junctions if SMC-to-SMC gap junctions have sufficient permeability.

Through synchronization of immediate neighbors, a large population can be

synchronized. For example, oscillatory $\mathrm{IP}_{3}$ can be induced by various isoforms of $\mathrm{Ca}^{2+}-$

dependent PLC, or even by $V_{\mathrm{m}}$ modulation of $\mathrm{IP}_{3}$ release (Imtiaz, von der Weid, and van

Helden 2009). $\mathrm{IP}_{3}$ can diffuse through gap junctions and modulate RyR or $\mathrm{IP}_{3} \mathrm{Rs}$. In

current-mediated synchronization, oscillatory $V_{\mathrm{m}}$ needs to be generated through

membrane channels that depend directly or indirectly on $\mathrm{Ca}^{2+}$. Current can spread 
relatively fast and over long distances and can modulate a population of cells through its action on VOCCs. A prerequisite for achieving synchronization is that the net effect of depolarizing and hyperpolarizing currents yields $V_{\mathrm{m}}$ oscillations in phase with the $\mathrm{Ca}^{2+}$ oscillations. Oscillatory $V_{\mathrm{m}}$ can be generated by $\mathrm{Ca}^{2+}$ dependent channels such as the $\mathrm{NSC}$, the $\mathrm{BK}_{\mathrm{Ca}}$ and the $\mathrm{Cl}_{\mathrm{Ca}}$.

The model suggests that coordinating signals are likely to be weak and superimposed on larger constant components. Synchronization is determined not by the relative magnitude of competing pathways but by their sensitivity to $\mathrm{Ca}^{2+}$ fluctuations. Thus, the component (i.e., an ion channel) that is affected the most by a system perturbation (i.e., agonist stimulation or channel block) may not necessarily be the component that allows synchronization. For example, VOCC block can prevent vasomotion by changing ionic balances and the ability of a cell for self-sustained oscillations and not necessarily by a direct effect on the synchronizing pathway. PLC inhibition is similarly inadequate to experimentally probe the role of $\mathrm{IP}_{3}$ in synchronization, because it prevents NE-induced signaling (Lamboley et al. 2005). A molecular $\mathrm{IP}_{3}$ buffer that does not change the steady state component of free $\mathrm{IP}_{3}$ would be more appropriate (Politi et al. 2006). [IP 3 buffering that attenuates $\mathrm{IP}_{3}$ oscillations can approximate selective block of intercellular $\mathrm{IP}_{3}$ diffusion]. An appropriate experimental intervention to probe "current-mediated synchronization" would be to clamp $V_{\mathrm{m}}$ to mean oscillatory value along the entire vessel segment. Clamping of $V_{\mathrm{m}}$ would effectively block electrical coupling. However, such interventions are difficult to perform experimentally. For these reasons experimental determination of the pathways contributing to synchronization can be very challenging. An ideal intervention should target a specific component or species and prevent its 
oscillation without modifying mean activity and self-sustained oscillations. Moreover, combinations of different targets should be used to elucidate potential synergies or redundancies under various stimuli. Such scenarios can be easily examined in silico. Through our simulations we evaluated the synchronizing potential of three major mechanisms.

\subsubsection{Diffusion mediated synchronization}

\subsubsection{Synchronization mediated by $\mathrm{Ca}^{2+}$ diffusion}

$\mathrm{Ca}^{2+}$ diffusion through nonselective SMC-SMC gap junctions is the most basic and direct pathway to provide coordination of the intracellular oscillators in neighboring cells. In a series of models for vasomotion in RMA, intercellular $\mathrm{Ca}^{2+}$ flux was the only significant mechanism for cell synchronization (Koenigsberger et al. 2004; Koenigsberger et al. 2005, 2006; Koenigsberger, Sauser, and Meister 2005). Other studies, however, have argued against $\mathrm{Ca}^{2+}$ diffusion as a synchronizing signal in RMA. Sell et al. estimated the $\mathrm{Ca}^{2+}$ propagation velocity in single SMC to be of $25 \mu \mathrm{m} / \mathrm{s}$ and concluded that it is too slow for intercellular $\mathrm{Ca}^{2+}$ synchronization (Sell, Boldt, and Markwardt 2002). Jacobsen and coworkers have also argued that the diffusion of $\mathrm{Ca}^{2+}$ (as well as of other second messengers) is too slow and that the intercellular $\mathrm{Ca}^{2+}$ flux is too small (Jacobsen et al. 2007). In their model, $\mathrm{Ca}^{2+}$ fluxes had no effect on wave pattern at the control gap junction permeability $P=5 \times 10^{-8} \mathrm{~m} / \mathrm{s}$ as well as for values up to $500 \times 10^{-8}$ $\mathrm{m} / \mathrm{s}$, beyond which cells behaved effectively like single cell.

Unfortunately, the intercellular permeability for $\mathrm{Ca}^{2+}$ has not been experimentally derived and previous theoretical studies have used values that differ by two to three orders of magnitude. In our models, we described $\mathrm{Ca}^{2+}$ fluxes based on electrochemical 
gradient and $\mathrm{Ca}^{2+}$ permeability is estimated from the intercellular gap junctional resistance $\left(R_{\mathrm{gg}}\right) \cdot R_{\mathrm{gj}}$ is a parameter that has been previously estimated from electrical and observational data. $\mathrm{Ca}^{2+}$ permeabilities values utilized in this study are in agreement with the permeabilities used by Jacobsen and coworkers (Jacobsen et al. 2007). Thus, it is not surprising that a similar conclusion was reached regarding the role of $\mathrm{Ca}^{2+}$ diffusion in cell synchronization. On the other hand, $P_{\mathrm{Ca}}$ value is uncharacteristically high in the studies that suggest an important role for $\mathrm{Ca}^{2+}$ diffusion (Koenigsberger et al. 2004). It can be argued that in lumped cell models, lacking spatial resolution intracellularly, an "effective" $\mathrm{Ca}^{2+}$ permeability larger than actual gap junction permeability may account for regenerative mechanisms in $\mathrm{Ca}^{2+}$ spread. However, the SMC model of Jacobsen et al. accounts for intracellular spatial resolution and CICR waves, and still intercellular $\mathrm{Ca}^{2+}$ diffusion was not important unless nonphysiological coupling was assumed (i.e., $R_{\mathrm{gj}}$ below $1 \mathrm{M} \Omega$ ). Better experimental data is required for a more conclusive determination.

\subsubsection{Synchronization mediated by $I P_{3}$ diffusion}

Model simulations show that intercellular $\mathrm{IP}_{3}$ diffusion can contribute to intercellular synchronization. If the $\mathrm{IP}_{3}$ coupling is sufficient to coordinate immediate neighbors, it can also synchronize a larger population (Figure 5.7). In the model, a contributing role for $\mathrm{IP}_{3}$ could be seen for $P_{\mathrm{IP} 3}^{\mathrm{SMC}-\mathrm{SMC}}$ values as low as $0.058 \mathrm{pL} / \mathrm{s}$. This value is below our control permeability value $(0.53 \mathrm{pL} / \mathrm{s})$. For $R_{\mathrm{gj}}^{\mathrm{SMC}-\mathrm{SMC}}=87.4 \mathrm{M} \Omega$, we estimate an ionic permeability of $P_{\mathrm{S}}^{\mathrm{SMC}-\mathrm{SMC}}=0.015 \mathrm{pL} / \mathrm{s}$ (section 7.1.1) and comparable permeabilities for ions and $\mathrm{IP}_{3}$ are possible. Thus, $P_{\mathrm{IP} 3}^{\mathrm{SMC}-\mathrm{SMC}}$ in the range of $0.01-0.5 \mathrm{pL} / \mathrm{s}$ is expected. We conclude that the contribution of $\mathrm{IP}_{3}$ in cell synchronization is conditional to a sufficient 
$\mathrm{IP}_{3}$ permeability and adequate cell coupling in the $\mathrm{SMC}$ layer. $\mathrm{IP}_{3}$ oscillations associated with $\mathrm{Ca}^{2+}$ oscillations is a necessary condition for $\mathrm{IP}_{3}$-mediated synchronization. $\mathrm{Ca}^{2+}$ oscillations may be accompanied by $\mathrm{IP}_{3}$ oscillations in various cell types, but further evidence is required to confirm such dependence in the vasculature (Politi et al. 2006; Bartlett et al. 2005). In RMA, IP 3 oscillations can be generated by $\alpha_{1}$-adrenoreceptors and $\mathrm{Ca}^{2}$-dependent PLC activity (Clarke et al. 2008). [Agonist-independent isoforms of PLC that was not explicitly described in the model can also contribute to $\mathrm{IP}_{3}$ oscillations in RMA SMCs (Lamboley et al. 2005; LaBelle and Polyak 1996)]. In the model of Koenigsberger et al. (Koenigsberger et al. 2004), the amplitude of $\mathrm{IP}_{3}$ oscillations was predicted to be too small to coordinate $\mathrm{Ca}^{2+}$ oscillations. Although there are no direct measurements, indirect experimental evidence suggests that $\mathrm{IP}_{3}$ may oscillate significantly in RMA SMCs during vasomotion. $\mathrm{EC} \mathrm{Ca}^{2+}$ has been observed to oscillate in phase with $\mathrm{SM} \mathrm{Ca}^{2+}$, and the more likely explanation for this behavior is the diffusion of oscillatory $\mathrm{IP}_{3}$ from SM to the endothelium (Schuster et al. 2001; Lamboley et al. 2005). [Movement of $\mathrm{IP}_{3}$, rather than $\mathrm{Ca}^{2+}$ between cells seems to play a more significant role in $\mathrm{Ca}^{2+}$ wave propagation and $\mathrm{IP}_{3}$ diffuses faster than $\mathrm{Ca}^{2+}$. Consistently with these data, our model predicts that $\mathrm{IP}_{3}$ has the potential to play an important role in synchronization. In some $\mathrm{SMCs}, \mathrm{IP}_{3}$ synthesis may be $V_{\mathrm{m}}$-dependent (Itoh et al. 1992; Imtiaz, Smith, and van Helden 2002). This provides an alternative mechanism for $\mathrm{IP}_{3}$ oscillations and thus, could allow for a contribution of $\mathrm{IP}_{3}$ in synchronization independently of the $\mathrm{Ca}^{2+}$-dependent PLC pathway kinetics. 


\subsubsection{Electrically mediated synchronization}

\subsubsection{Synchronization mediated by electrical coupling}

Ionic coupling through gap junctions transmits $V_{\mathrm{m}}$ changes from one cell to another. This, however, can have a synchronizing effect only if $\mathrm{Ca}^{2+}$ elevation generates adequate and in-phase $V_{\mathrm{m}}$ depolarization. The transmitted depolarization will then activate VOCCs and increase $\mathrm{Ca}^{2+}$ in neighboring cells. $\mathrm{Cl}_{\mathrm{Ca}}$ channels have been proposed to mediate the necessary $\mathrm{Ca}^{2+}$ and $V_{\mathrm{m}}$ coupling in vasomotion and electric current was suggested as the only signal fast enough (i.e., $>1 \mathrm{~cm} / \mathrm{s}$ ) to synchronize several millimeters long vessels (Nilsson and Aalkjaer 2003; Matchkov, Aalkjaer, and Nilsson 2004). Nonetheless, analysis of experimental data and model results indicate that additional mechanisms may be contributing. In vasomotion, SMC $V_{\mathrm{m}}$ oscillations are typically in phase with $\mathrm{Ca}^{2+}$ and proceed oscillations in SMC tension (Aalkjaer and Nilsson 2005). However, $V_{\mathrm{m}}$ fluctuations are not always synchronous to diameter changes (Schuster et al. 2004), and a theoretical analysis even predicts $V_{\mathrm{m}}$ oscillations in antiphase with $\mathrm{Ca}^{2+}$ oscillations (Koenigsberger et al. 2004). Furthermore, in $\mathrm{RMA} \mathrm{Ca}^{2+}$ oscillations are normally generated by the SR and $V_{\mathrm{m}}$ oscillations are secondary to the $\mathrm{Ca}^{2+}$ oscillations. Thus, $V_{\mathrm{m}}$ may have an arbitrary phase shift relative to $\mathrm{Ca}^{2+}$, depending on the type and number of $\mathrm{Ca}^{2+}$ modulated channels. This suggests that electrical coupling may not always have a synchronizing effect.

DAG-activated TRPC-like nonselective cation channels are expressed in RMA SMCs and mediate depolarizing $\mathrm{Na}^{+}$influx during $\alpha_{1}$-adrenergic stimulation (Hill et al. 2006). Our model predicts that NSC channels are good candidates for generating synchronizing $V_{\mathrm{m}}$ changes, assuming that the $\mathrm{Ca}^{2+}$ dependence of PLC leads to oscillatory DAG. Indeed, 
synchronous oscillations of DAG, $\mathrm{IP}_{3}$ and $\mathrm{Ca}^{2+}$ induced by NE were measured in Chinese hamster ovary cell culture (Bartlett et al. 2005). Unlike some vessels with spontaneous vasomotion, RMAs require depolarization that opens VOCCs and maintains mean $\mathrm{Ca}^{2+}$ levels within the oscillatory window (Schuster et al. 2004). In the model, NE opens NSC channels, reduces significantly membrane resistance and depolarizes SMCs in agreement with experiments (Sell, Boldt, and Markwardt 2002). Thus, the agonist-activated NSC channels have a major effect on the electrical responses that has not been examined in previous models (Koenigsberger et al. 2004; Jacobsen et al. 2007). Mechanosensitive NSC channels modulated by wall stress were predicted to have only minor synchronizing effect (Koenigsberger et al. 2006). The effectiveness of $\mathrm{NSC}$ and $\mathrm{Cl}_{\mathrm{Ca}}$ channels to synchronize depends on the amplitude of induced $V_{\mathrm{m}}$ oscillations, (i.e., the $\mathrm{Ca}^{2+}$ dependent variation of conductance has to be significant compared to total membrane resistance). Under the conditions of Figure 5.2-B, where EC and SMC layers are moderately stimulated by Ach and NE, the fluctuations in $\mathrm{NSC}$ and $\mathrm{Cl}^{-}$conductances are similar, but the predicted oscillatory NSC and $\mathrm{Cl}^{-}$currents differ significantly (i.e. $15 \mathrm{pA}$ and $2.5 \mathrm{pA}$, respectively). This is attributed to a larger electrochemical gradient for $\mathrm{Na}^{+}$ ions. Consequently, clamping NSC channels resulted in large amplitude antiphase $V_{\mathrm{m}}$ oscillations and desynchronization (Figure 5.2-B), while clamping $\mathrm{Cl}^{-}$currents had no significant effect under these conditions.

Experimental evidence supports a role for $\mathrm{Cl}^{-}$current in vasomotion. Exogenous cGMP administration in endothelium-denuded vessels restored vasomotion, suggesting a role for cGMP dependent $\mathrm{Cl}^{-}$channels (Peng et al. 2001). In intact vessels, $\mathrm{Cl}_{\mathrm{Ca}}$ blockade has not consistently abolished vasomotion (i.e. in intact small RMA, vasomotion was 
inhibited by $\mathrm{Cl}^{-}$substitution, but not by DIDS and $\mathrm{Zn}^{2+}$ (blockers of cGMP-dependent $\mathrm{Cl}_{\mathrm{Ca}}$ channels) (Boedtkjer et al. 2008)). In a recent study however, transfecting rat mesenteric small arteries in vivo with siRNA specifically targeting bestrophin-3, inhibits cGMP dependent $\mathrm{Cl}_{\mathrm{Ca}}$ current and abolishes vasomotion in isolated arteries (Broegger et al. 2011). Thus, although $\mathrm{Cl}^{-}$current is the only synchronizing mechanism that has been experimentally tested, the conditions under which $\mathrm{Cl}^{-}$channels induce vasomotion, particularly in the presence of endothelium, is a topic of continuing investigation. Our simulations suggest that exogenous cGMP administration can enhance the potential for synchronization by activation of $\mathrm{Cl}_{\mathrm{Ca}}$ and this might be the predominant synchronizing mechanism under some conditions (Figures 5.2-C and D). When endothelium derived factors, however, lead to endogenous cGMP production, the cGMP increase is not necessarily as effective as in EC denuded vessels (Figure 5.6-A). According to the model, this is attributed to other $\mathrm{EC}$-induced pathways that interfere with $\mathrm{Cl}_{\mathrm{Ca}}$ mediated synchronization (i.e. the parallel activation of $\mathrm{BK}_{\mathrm{Ca}}$ by $\mathrm{NO}$ ) and to a different $\mathrm{SM} V_{\mathrm{m}}$ that alters sensitivity of currents to $\mathrm{Ca}^{2+}$ changes. Simulations also show that $\mathrm{Cl}_{\mathrm{Ca}}$ blockade can inhibit synchronization (Figure 5.6-B) when $\mathrm{Cl}_{\mathrm{Ca}}$ clamping has no effect (Figure 5.6A). Thus, under some conditions, blocking $\mathrm{Cl}_{\mathrm{Ca}}$ may be able to inhibit vasomotion even when $\mathrm{Cl}^{-}$current is not the synchronizing signal. Model simulations do not prove a limited role for $\mathrm{Cl}_{\mathrm{Ca}}$ in synchronization in intact vessels. They show however, that under some conditions $\mathrm{Cl}_{\mathrm{Ca}}$ may not be the predominant synchronizing pathway even when exogenous cGMP administration or $\mathrm{Cl}_{\mathrm{Ca}}$ blockade can affect vasomotion. 
In the model, a synchronizing mechanism has to overcome cellular heterogeneity and the effect of $\mathrm{BK}_{\mathrm{Ca}}$ current which tends to counteract NSC and $\mathrm{Cl}_{\mathrm{Ca}}$ channels. This prediction is consistent with the findings that blockade of $\mathrm{BK}_{\mathrm{Ca}}$ with $\mathrm{IbTx}$ or $\mathrm{Kv}$ with 4AP did not eliminate vasomotion, and blockade of $\mathrm{K}^{+}$channels with TEA in some vessel types promotes vasomotion (Mauban and Wier 2004; Aalkjaer and Nilsson 2005). TEA was suggested to enhance vasomotion by increasing membrane resistance and/or gap junction formation. In a model with identical self-oscillatory SMCs in which $\mathrm{BK}_{\mathrm{Ca}}$ currents are dominant, electrical coupling tends to produce clusters of synchronous oscillations. The clusters may consist of adjacent as well as distant cells, and there can be many configurations depending on initial perturbation of the system. Figure 5.8 shows cluster formation in a population of eighty SMCs arranged into a cylinder. Such clustering can arise whenever $\mathrm{Ca}^{2+}$ increase in one cell tends to reduce $\mathrm{Ca}^{2+}$ in other cells. In this particular case, $\mathrm{Ca}^{2+}$ elevation activates $\mathrm{BK}_{\mathrm{Ca}}$ channels and repolarizes $V_{\mathrm{m}}$, which in turn closes VOCC in neighboring cells due to electrical coupling. For non-identical cells or in the presence of noise, the formation of clusters decreases as the heterogeneity increases, until no patterns are formed (Koenigsberger, Sauser, and Meister 2005). The desynchronizing effect of $\mathrm{BK}_{\mathrm{Ca}}$ in the model is significant and $\mathrm{BK}_{\mathrm{Ca}}$ clamp increased synchronization under most stimulatory conditions examined.

\subsubsection{Role of endothelium}

The effect of endothelium on vasomotion remains controversial and both synchronizing and desynchronizing effects of endothelium have been reported even in the same vessel types, including in RMA (Mauban and Wier 2004; Peng et al. 2001; Rahman et al. 2005; Sell, Boldt, and Markwardt 2002; Lamboley et al. 2003; Aalkjaer and Nilsson 
2005). Some of these discrepancies can be explained by the modulatory role of ECs on mean $\mathrm{SM} \mathrm{Ca}^{2+}$ and transitions between oscillatory and nonoscillatory domains, similar to the effect of pressure (Mauban and Wier 2004; Koenigsberger et al. 2005, 2006). A synchronizing effect of the endothelium through some unidentified mechanism was also suggested by Rahman et al. (Rahman et al. 2005) based on the finding that cGMP produced only partial synchronization in endothelium denuded RMA, while intact endothelium allowed full synchronization even with L-NAME and ODQ.

According to our model, the inconsistent role of endothelial layer may also result from multiple effects of endothelium-derived factors on the synchronization mechanisms and their sensitivity to experimental conditions. Endothelium has relatively low electrical resistance and thus can provide global coupling (mean field) for SMCs through myoendothelial gap junctions. However, this can enhance or attenuate synchronization depending on the phase between SMC $V_{\mathrm{m}}$ and $\mathrm{Ca}^{2+}$ as shown and discussed above. Inhibition of $\mathrm{EDHF}\left(\mathrm{IK}_{\mathrm{Ca}}\right.$ and $\mathrm{SK}_{\mathrm{Ca}}$ channels) can desynchronize $\mathrm{Ca}^{2+}$ oscillations by depolarizing $\mathrm{SM} V_{\mathrm{m}}$ and activating $\mathrm{BK}_{\mathrm{Ca}}$ channels (Figure 5.3-A). NO can potentially induce vasomotion by bringing $\mathrm{Ca}^{2+}$ levels into the oscillatory window (Figure 5.3-B). However, the synchronizing effect of NO is complex and difficult to predict. NO can activate directly or through sGC/cGMP depolarizing and hyperpolarizing currents that can affect synchronization (Figure 5.3-D). NO can potentially have steady-state and oscillatory components. The model predicts that an oscillatory component of NO can influence synchronization by its effect on $\mathrm{BK}_{\mathrm{Ca}}$ (Figure 5.3-C). The effect depends on the phase shift between $\mathrm{SM} \mathrm{Ca}^{2+}$ and NO. 


\subsection{Model limitations}

The model contains a number of simplifying assumptions and estimates for several parameter values which limit model's predictive ability. Some of these assumptions can significantly affect model predictions. The primary purpose of the presented simulations is to demonstrate the feasibility of a synchronizing pathway and less emphasis should be placed on the parameter values and conditions where vasomotion occurs (i.e. agonist concentrations etc). Specific limitations of the model are discussed in the Supplement section (Section 5.8).

\subsection{Conclusions}

The model predicts several physiologically feasible pathways for synchronization of $\mathrm{SMCs}$ in vasomotion. These include current generated through $\mathrm{Cl}_{\mathrm{Ca}}$ and $\mathrm{NSC}$ channels, or pathways that involve the intercellular diffusion of $\mathrm{IP}_{3}$ and perhaps of $\mathrm{Ca}^{2+}$ (Figure 5.4). Their relative importance is sensitive to many parameters and stimulatory conditions. Competing pathways exist and synchronization is determined by the relative strength of each pathway (i.e., the pathway's sensitivity to $\mathrm{Ca}^{2+}$ fluctuations). This may explain controversies in experimental and theoretical results about vasomotion. Despite the increased complexity of the model and the utilization of a large amount of electrophysiological data, many aspects of $\mathrm{Ca}^{2+}$ signaling remain unclear and have not been incorporated. The further development of models and experiments, in parallel, will enable us to elucidate the mechanisms underlying the phenomenon of vasomotion. 


\subsection{Supplement}

\subsubsection{Oscillations in SMCs}

Figure 5.5-A shows the oscillatory domain for an isolated SMC (solid line). The model generates self-sustained $\mathrm{Ca}^{2+}$ oscillations whenever the cytosolic $\mathrm{SMC} \mathrm{Ca}^{2+}$ level is in the range of $160-290 \mathrm{nM}$. The corresponding norepinephrine (NE) concentrations to achieve such levels depend on the presence and stimulation state of the EC as the endothelium modulates SMC $\mathrm{Ca}^{2+}$ levels. Thus, a stimulated endothelium yields a significantly larger NE concentration window for oscillations (dotted line). In a multicellular vessel, the synchronization domain will be a subset of the oscillatory domain because unsynchronized oscillations may occur.

(A)

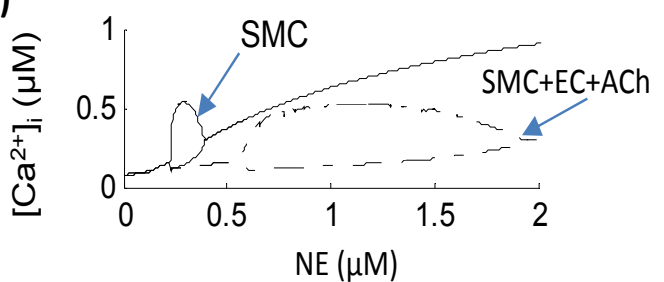

(C)

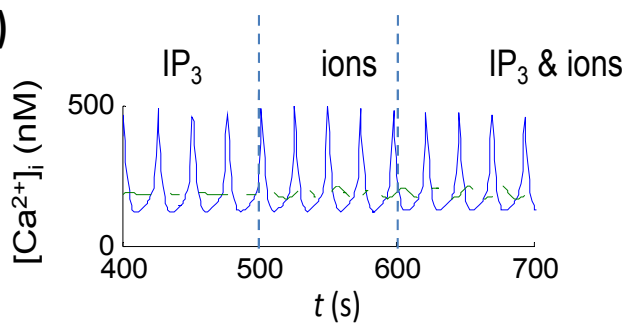

(B) 0 - isolated, $x$ - coupled, ${ }^{*}$ - synchronized cells

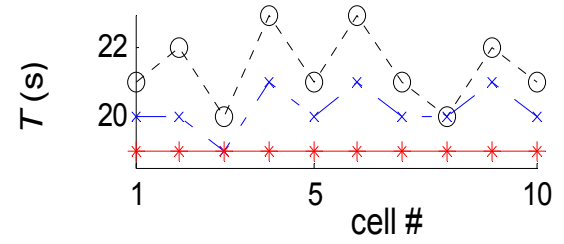

(D)

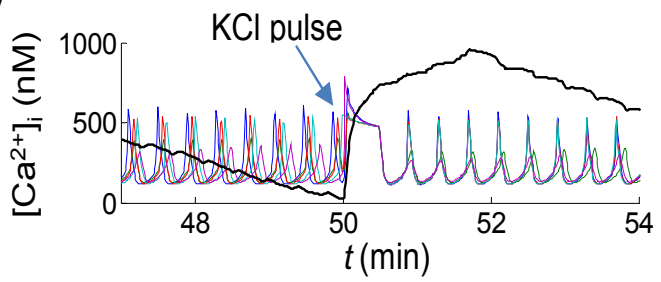

Figure 5.5 (A) Bifurcation diagrams of $\mathrm{Ca}^{2+}$ versus NE for single SMC model without EC (solid line) and with EC stimulated by Ach (dashed-dotted line). (B) Representative distribution of oscillation periods in a model with 10 oscillatory SMCs before coupling (o), after coupling without synchronization $(\times)$ and with synchronization $(*)$. (C) Forced $\mathrm{Ca}^{2+}$ oscillations in non-oscillatory SMC induced by $\mathrm{IP}_{3}$ and electrical coupling with an oscillatory SMC. (D) Unsynchronized $\mathrm{Ca}^{2+}$ oscillations induced by $0.3 \mu \mathrm{M}$ NE were synchronized transiently by a short $10 \mathrm{~ms}$ pulse of high extracellular $\mathrm{K}^{+}(35 \mathrm{mM})$ resetting simultaneously all SMC oscillators. 
Figure 5.5-B shows representative distribution of oscillation periods in a multicellular model comprised of 10 oscillatory SMCs before and after intercellular coupling. A distribution in the natural frequencies of $\mathrm{Ca}^{2+}$ oscillations accounts for biological variability and was achieved as described in the methods section. The ability of cells to synchronize decreased with increasing dispersion in their natural frequencies. In our simulations, the ratio of minimum to maximum period was maintained bellow 0.9 in all simulations. Frequency differences of this magnitude have been observed experimentally in two oscillatory regions during beating vasomotion (26). When cells are coupled, oscillatory periods are modified (blue line) and under some conditions synchronization may occur and cells assume a common, often reduced, period of $\mathrm{Ca}^{2+}$ oscillations (red line).

Figure 5.5-C examines the strength of coupling (i.e., the amplitude of $\mathrm{Ca}^{2+}$ oscillations in one cell due to the $\mathrm{Ca}^{2+}$ oscillations in another cell). A non-oscillatory SMC was created (i.e. by appropriate selection of store parameters) and was coupled to an oscillatory SMC. The non-oscillatory SMC exhibited only small forced $\mathrm{Ca}^{2+}$ oscillations (dotted line), consistently with the observation that in the artery wall $\mathrm{Ca}^{2+}$ transients in one SMC do not typically produce $\mathrm{Ca}^{2+}$ transients in a neighboring cell (26). In the oscillatory cell, $\mathrm{Ca}^{2+}$ generates $\mathrm{IP}_{3}$ and DAG oscillations through $\mathrm{Ca}^{2+}$ feedback on the PLC, and $V_{\mathrm{m}}$ oscillations through DAG-dependent NSC channels and $\mathrm{Ca}^{2+}$-dependent $\mathrm{BK}_{\mathrm{Ca}}$ and $\mathrm{Cl}_{\mathrm{Ca}}$ channels. The intercellular $\mathrm{IP}_{3}$ coupling gives rise to small $\mathrm{IP}_{3}$ oscillations in the non-oscillatory cell, opening of $\mathrm{IP}_{3} \mathrm{Rs}$ and periodic $\mathrm{Ca}^{2+}$ release. Electrical coupling imposes $V_{\mathrm{m}}$ oscillations in the secondary cell and modules activity of VOCC. The net 
effect of these mechanisms is not significant enough to induce forced oscillations in the second cell.

A rapid addition of an agonist, like $\mathrm{NE}, \mathrm{K}^{+}$or $\mathrm{NO}$, can cause simultaneous depolarization/repolarization of the whole population of SMCs, and thus may initiate a transient $\mathrm{Ca}^{2+}$ synchronization. The effect of a short pulse of extracellular $\mathrm{K}^{+}(35 \mathrm{mM}$ $\mathrm{KCl}$ ) applied uniformly to several SMCs is shown in Figure 5.5-D. Unsynchronized oscillations were transiently synchronized by the short pulse, consistently with experiments (5). The synchronization was caused by phase resetting of the $\mathrm{Ca}^{2+}$ oscillators. To ensure that $\mathrm{Ca}^{2+}$ coordination is mediated by intercellular communication (and is not merely a result of resetting), sufficiently long times must be considered in simulations and experiments.

\subsubsection{Role of CICa when ECs are present}

In the presence of endothelium and moderate levels of Ach and NE stimulations (i.e., Ach $=1$ a.u.; $\mathrm{NE}=0.8 \mu \mathrm{M}$ ), clamping $\mathrm{Cl}_{\mathrm{Ca}}$ channels did not elicit desynchronization (Figure 5.6-A). To better compare model predictions with experimental data, we also performed simulations with total block of $\mathrm{Cl}_{\mathrm{Ca}}$ currents. Under the same stimulatory conditions, total inhibition of $\mathrm{Cl}_{\mathrm{Ca}}$ channels desynchronized a population of $\mathrm{SMCs}$ (Figure 5.6-B). The result was not consistent in all of the examined SMC sets with random oscillatory frequencies. 

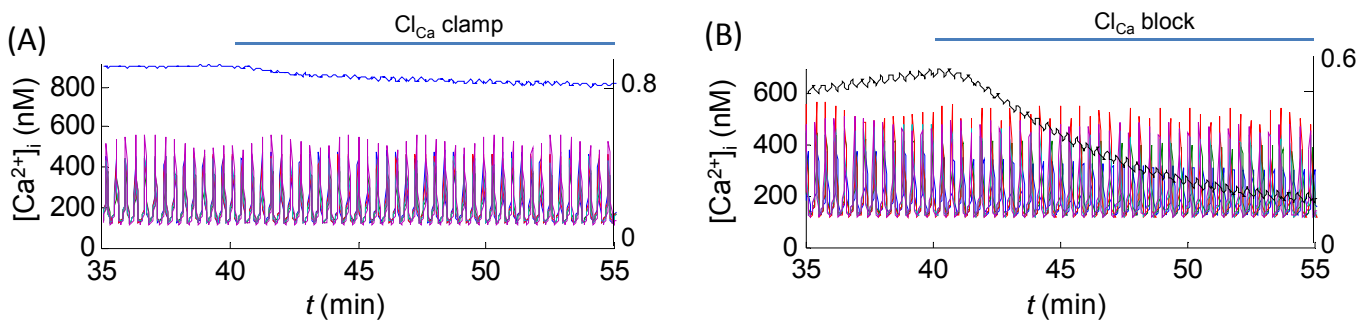

Figure 5.6 A population of five SMCs and five ECs is stimulated by NE $(0.8 \mu \mathrm{M})$ and Ach (1 a.u.). $\mathrm{Ca}^{2+}$ oscillations in the SMCs are shown. Clamping $\mathrm{Cl}_{\mathrm{Ca}}$ channels did not elicit desynchronization (A), but total block of $\mathrm{Cl}_{\mathrm{Ca}}$ current can lead to loss of synchronization (B).

\subsubsection{Simulations with large ensembles}

Representative simulations with large number of cells arranged into a cylinder (vessel wall) are shown in Figures 5.7 and 5.8. Each SMC within the cylinder is coupled to its four neighbors through $R_{\mathrm{gj}}=87.4 \mathrm{M} \Omega$. In Figure 5.7, SMCs are coupled to ECs arranged perpendicularly to SMCs through the total myoendothelial $R_{\mathrm{gj}}=900 \mathrm{M} \Omega$ per SMC.

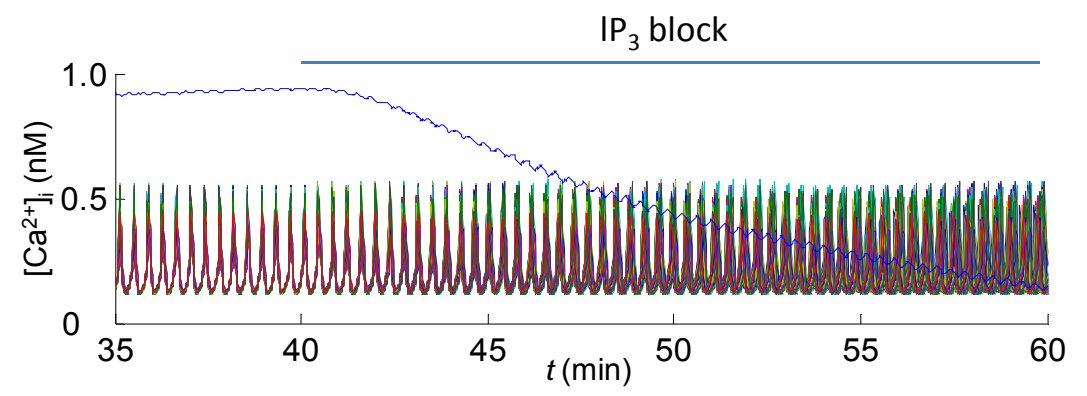

Figure 5.7 Simulations in a population of 80 SMCs and 80 underlying ECs arranged into a cylinder. In the presence of $0.8 \mathrm{NE}$ and 1 a.u. Ach, inhibition of intercellular $\mathrm{IP}_{3}$ diffusion desynchronized the oscillations, similarly to results with five SMCs from Figure 5.2-A.

\subsubsection{Model Limitations}

In this study regular $\mathrm{Ca}^{2+}$ oscillations are generated by RyRs and slow refilling of SR as in (Koenigsberger et al. 2004). However, the exact mechanism and even the deterministic nature of the oscillations has not been definitively resolved, and oscillators based on $\mathrm{IP}_{3}$ Rs have also been used in other models (Jacobsen et al. 2007). 

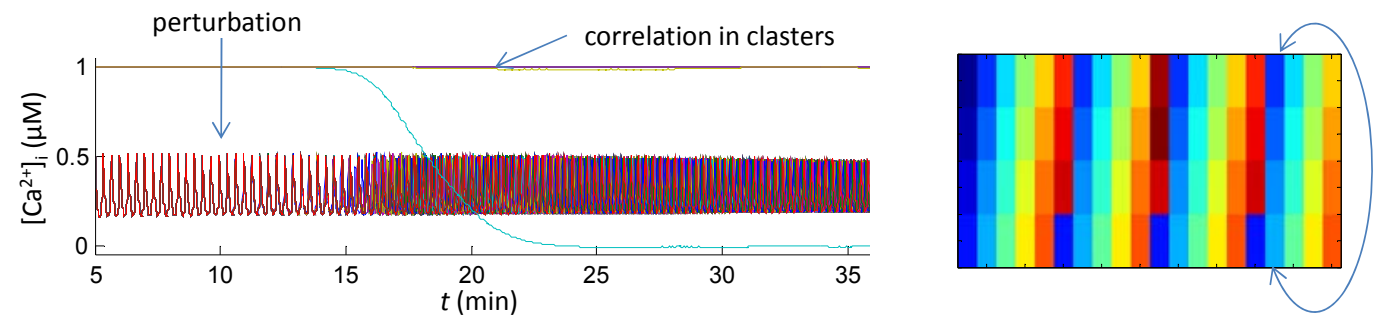

Figure 5.8 Cluster formation in a population of 80 identical SMCs arranged into a cylinder. When stimulated by $0.4 \mu \mathrm{M}$ NE from the same initial conditions, the cells produce in-phase oscillations (left). A short local perturbation of the system (10\% NE increase for 1 minute at cell \#1) destabilizes the oscillations and initiates formation of clusters (right). Cells with the same color are in-phase. The curve indicates circular boundary conditions.

Some studies suggested that the natural frequencies of SMCs within a vascular segment are very similar and only noise contributes significant variability in the system, whereas others reported that the frequencies of individual SMCs may differ significantly (Koenigsberger et al. 2004; Jacobsen et al. 2007; Jacobsen et al. 2008). Furthermore, the period of vasomotion in RMA varies from few seconds (Peng et al. 2001; Matchkov et al. 2004; Boedtkjer et al. 2008) to almost half a minute (Mauban and Wier 2004; Sell, Boldt, and Markwardt 2002; Seppey et al. 2008, 2010). In this study, oscillatory frequency was predicted based on previously determined kinetic constants and parameter values, and it falls within the range of previously reported values. Significant differences in frequency can potentially affect the synchronization properties of the system, thus, future studies need to examine the effect of oscillation frequency on synchronization.

Our lumped model does not account for heterogeneous intracellular distribution of RyRs and $\mathrm{IP}_{3} \mathrm{Rs}$ and their compartmentalization with membrane channels. All cellular components experience the same cytosolic $\mathrm{Ca}^{2+}$ concentration, disregarding possible differential activation of $\mathrm{PLC}, \mathrm{Cl}_{\mathrm{Ca}}$ and $\mathrm{BK}_{\mathrm{Ca}}$. Although vasomotion is usually associated with global (whole cell) $\mathrm{Ca}^{2+}$ oscillations, intracellular $\mathrm{Ca}^{2+}$ waves may play a role in 
initiation and maintenance of vasomotion (Jacobsen et al. 2007). A limitation of lumped models is that they do not account for intracellular diffusion of species $\left(\mathrm{Ca}^{2+}\right.$ and $\left.\mathrm{IP}_{3}\right)$ and may not accurately reproduce inter-cellular wave (Seppey et al. 2010). Continuum, spatially-distributed cellular models are needed to capture some of these phenomena.

Mechanical feedback on SM and endothelium may also play a role, but predictions require integration of this model with biomechanical models. NE can initiate vasomotion in both endothelium-denuded and intact RMA. In the model synchronized oscillations in the presence of EC required background Ach stimulation. The prestimulation with Ach compensated for changes in resting $V_{\mathrm{m}}$ caused by EC coupling, and may account for background $\mathrm{IP}_{3}$ levels and resting EC activity under experimental conditions.

\subsection{References}

Aalkjaer, C., and H. Nilsson. 2005. Vasomotion: cellular background for the oscillator and for the synchronization of smooth muscle cells. Br J Pharmacol 144 (5):60516.

Bartlett, P. J., K. W. Young, S. R. Nahorski, and R. A. Challiss. 2005. Single cell analysis and temporal profiling of agonist-mediated inositol 1,4,5-trisphosphate, $\mathrm{Ca} 2+$, diacylglycerol, and protein kinase $\mathrm{C}$ signaling using fluorescent biosensors. $J$ Biol Chem 280 (23):21837-46.

Benham, C. D., T. B. Bolton, R. J. Lang, and T. Takewaki. 1986. Calcium-activated potassium channels in single smooth muscle cells of rabbit jejunum and guineapig mesenteric artery. J Physiol 371:45-67.

Berridge, M. J. 2008. Smooth muscle cell calcium activation mechanisms. J Physiol 586 (Pt 21):5047-61.

Boedtkjer, D. M., V. V. Matchkov, E. Boedtkjer, H. Nilsson, and C. Aalkjaer. 2008. Vasomotion has chloride-dependency in rat mesenteric small arteries. Pflugers Arch 457 (2):389-404.

Broegger, T., J. C. Jacobsen, V. Secher Dam, D. M. Boedtkjer, H. Kold-Petersen, F. S. Pedersen, C. Aalkjaer, and V. V. Matchkov. 2011. Bestrophin is important for the rhythmic but not the tonic contraction in rat mesenteric small arteries. Cardiovasc Res 91 (4):685-93. 
Clarke, C. J., S. Forman, J. Pritchett, V. Ohanian, and J. Ohanian. 2008. Phospholipase C-deltal modulates sustained contraction of rat mesenteric small arteries in response to noradrenaline, but not endothelin-1. Am J Physiol Heart Circ Physiol 295 (2):H826-34.

Duling, B. R., R. W. Gore, R. G. Dacey, Jr., and D. N. Damon. 1981. Methods for isolation, cannulation, and in vitro study of single microvessels. Am J Physiol 241 (1):H108-116.

Gonzalez-Fernandez, J. M., and B. Ermentrout. 1994. On the origin and dynamics of the vasomotion of small arteries. Math Biosci 119 (2):127-67.

Haddock, R. E., and C. E. Hill. 2005. Rhythmicity in arterial smooth muscle. J Physiol 566 (Pt 3):645-56.

Hill, A. J., J. M. Hinton, H. Cheng, Z. Gao, D. O. Bates, J. C. Hancox, P. D. Langton, and A. F. James. 2006. A TRPC-like non-selective cation current activated by alpha 1adrenoceptors in rat mesenteric artery smooth muscle cells. Cell Calcium 40 (1):29-40.

Imtiaz, M. S., D. W. Smith, and D. F. van Helden. 2002. A theoretical model of slow wave regulation using voltage-dependent synthesis of inositol 1,4,5-trisphosphate. Biophys J 83 (4):1877-90.

Imtiaz, M. S., P. Y. von der Weid, and D. F. van Helden. 2009. Synchronization of Ca2+ oscillations: a coupled oscillator-based mechanism in smooth muscle. Febs J 277 (2):278-85.

Itoh, T., N. Seki, S. Suzuki, S. Ito, J. Kajikuri, and H. Kuriyama. 1992. Membrane hyperpolarization inhibits agonist-induced synthesis of inositol 1,4,5trisphosphate in rabbit mesenteric artery. J Physiol 451:307-28.

Jacobsen, J. C., C. Aalkjaer, V. V. Matchkov, H. Nilsson, J. J. Freiberg, and N. H. Holstein-Rathlou. 2008. Heterogeneity and weak coupling may explain the synchronization characteristics of cells in the arterial wall. Philos Transact A Math Phys Eng Sci 366 (1880):3483-502.

Jacobsen, J. C., C. Aalkjaer, H. Nilsson, V. V. Matchkov, J. Freiberg, and N. H. HolsteinRathlou. 2007. Activation of a cGMP-sensitive calcium-dependent chloride channel may cause transition from calcium waves to whole cell oscillations in smooth muscle cells. Am J Physiol Heart Circ Physiol 293 (1):H215-28.

Jacobsen, J. C., C. Aalkjaer, H. Nilsson, V. V. Matchkov, J. Freiberg, and N. H. HolsteinRathlou. 2007. A model of smooth muscle cell synchronization in the arterial wall. Am J Physiol Heart Circ Physiol 293 (1):H229-37. 
Kapela, A., A. Bezerianos, and N. M. Tsoukias. 2008. A mathematical model of Ca2+ dynamics in rat mesenteric smooth muscle cell: agonist and NO stimulation. $J$ Theor Biol 253 (2):238-60.

Kapela, A., A. Bezerianos, and N. M. Tsoukias. 2009. A mathematical model of vasoreactivity in rat mesenteric arterioles: I. Myoendothelial communication. Microcirculation 16 (8):694-713.

Kapela, A., S. Nagaraja, and N. M. Tsoukias. 2010. A mathematical model of vasoreactivity in rat mesenteric arterioles. II. Conducted vasoreactivity. Am J Physiol Heart Circ Physiol 298 (1):H52-65.

Koenigsberger, M., R. Sauser, J. L. Beny, and J. J. Meister. 2005. Role of the endothelium on arterial vasomotion. Biophys $J 88$ (6):3845-54.

Koenigsberger, M., R. Sauser, J. L. Beny, and J. J. Meister. 2006. Effects of arterial wall stress on vasomotion. Biophys $J 91$ (5):1663-74.

Koenigsberger, M., R. Sauser, M. Lamboley, J. L. Beny, and J. J. Meister. 2004. Ca2+ dynamics in a population of smooth muscle cells: modeling the recruitment and synchronization. Biophys $J 87$ (1):92-104.

Koenigsberger, M., R. Sauser, and J. J. Meister. 2005. Emergent properties of electrically coupled smooth muscle cells. Bull Math Biol 67 (6):1253-72.

LaBelle, E. F., and F. Polyak. 1996. Phospholipase C beta 2 in vascular smooth muscle. $J$ Cell Physiol 169 (2):358-63.

Lamboley, M., P. Pittet, M. Koenigsberger, R. Sauser, J. L. Beny, and J. J. Meister. 2005. Evidence for signaling via gap junctions from smooth muscle to endothelial cells in rat mesenteric arteries: possible implication of a second messenger. Cell Calcium 37 (4):311-20.

Lamboley, M., A. Schuster, J. L. Beny, and J. J. Meister. 2003. Recruitment of smooth muscle cells and arterial vasomotion. Am J Physiol Heart Circ Physiol 285 (2):H562-9.

Matchkov, V. V., C. Aalkjaer, and H. Nilsson. 2004. A cyclic GMP-dependent calciumactivated chloride current in smooth-muscle cells from rat mesenteric resistance arteries. J Gen Physiol 123 (2):121-34.

Matchkov, V. V., A. Rahman, H. Peng, H. Nilsson, and C. Aalkjaer. 2004. Junctional and nonjunctional effects of heptanol and glycyrrhetinic acid derivates in rat mesenteric small arteries. Br J Pharmacol 142 (6):961-72. 
Mauban, J. R., and W. G. Wier. 2004. Essential role of EDHF in the initiation and maintenance of adrenergic vasomotion in rat mesenteric arteries. Am J Physiol Heart Circ Physiol 287 (2):H608-16.

Nilsson, H., and C. Aalkjaer. 2003. Vasomotion: mechanisms and physiological importance. Mol Interv 3 (2):79-89, 51.

Parthimos, D., D. H. Edwards, and T. M. Griffith. 1999. Minimal model of arterial chaos generated by coupled intracellular and membrane Ca2+ oscillators. Am J Physiol 277 (3 Pt 2):H1119-44.

Parthimos, D., D. H. Edwards, and T. M. Griffith. 2003. Shil'nikov homoclinic chaos is intimately related to type-III intermittency in isolated rabbit arteries: role of nitric oxide. Phys Rev E Stat Nonlin Soft Matter Phys 67 (5 Pt 1):051922.

Parthimos, D., R. E. Haddock, C. E. Hill, and T. M. Griffith. 2007. Dynamics of a threevariable nonlinear model of vasomotion: comparison of theory and experiment. Biophys J 93 (5):1534-56.

Peng, H., V. Matchkov, A. Ivarsen, C. Aalkjaer, and H. Nilsson. 2001. Hypothesis for the initiation of vasomotion. Circ Res 88 (8):810-5.

Pikovsky, Arkady, Michael Rosenblum, and J. Kurths. 2001. Synchronization : a universal concept in nonlinear sciences, The Cambridge nonlinear science series ; 12. Cambridge: Cambridge University Press.

Politi, A., L. D. Gaspers, A. P. Thomas, and T. Hofer. 2006. Models of IP3 and Ca2+ oscillations: frequency encoding and identification of underlying feedbacks. Biophys $J 90$ (9):3120-33.

Rahman, A., V. Matchkov, H. Nilsson, and C. Aalkjaer. 2005. Effects of cGMP on coordination of vascular smooth muscle cells of rat mesenteric small arteries. $J$ Vasc Res 42 (4):301-11.

Schuster, A., M. Lamboley, C. Grange, H. Oishi, J. L. Beny, N. Stergiopulos, and J. J. Meister. 2004. Calcium dynamics and vasomotion in rat mesenteric arteries. $J$ Cardiovasc Pharmacol 43 (4):539-48.

Schuster, A., H. Oishi, J. L. Beny, N. Stergiopulos, and J. J. Meister. 2001. Simultaneous arterial calcium dynamics and diameter measurements: application to myoendothelial communication. Am J Physiol Heart Circ Physiol 280 (3):H108896.

Sell, M., W. Boldt, and F. Markwardt. 2002. Desynchronising effect of the endothelium on intracellular $\mathrm{Ca} 2+$ concentration dynamics in vascular smooth muscle cells of rat mesenteric arteries. Cell Calcium 32 (3):105-20. 
Seppey, D., R. Sauser, M. Koenigsberger, J. L. Beny, and J. J. Meister. 2008. Does the endothelium abolish or promote arterial vasomotion in rat mesenteric arteries? Explanations for the seemingly contradictory effects. J Vasc Res 45 (5):416-26.

Seppey, D., R. Sauser, M. Koenigsberger, J. L. Beny, and J. J. Meister. 2010. Intercellular calcium waves are associated with the propagation of vasomotion along arterial strips. Am J Physiol Heart Circ Physiol 298 (2):H488-96.

Silva, H. S., A. Kapela, and N. M. Tsoukias. 2007. A mathematical model of plasma membrane electrophysiology and calcium dynamics in vascular endothelial cells. Am J Physiol Cell Physiol 293 (1):C277-93. 
CHAPTER 6-SUMMARY OF THE DISSERTATION 
$\mathrm{Ca}^{2+}$ mobilization in vascular cells (SMCs and ECs) range from locally confined subcellular events to global whole-cell $\mathrm{Ca}^{2+}$ increases. Moreover, $\mathrm{Ca}^{2+}$ increases in individual cells may spread across the adjacent vascular cells and are regulated by a network of intracellular signaling pathways and complex inter-cellular interactions. Intracellular $\mathrm{Ca}^{2+}$ concentrations in the SMCs determine the vessel tone through modulation of the contractile apparatus (global $\mathrm{Ca}^{2+}$ rise in the SMCs produces vessel constriction). Under certain conditions, transient oscillatory $\mathrm{Ca}^{2+}$ changes in the SMCs can synchronize along the vessel segment to result in spontaneous oscillations in vessel diameter (vasomotion). On the other hand, ECs influence vessel tone regulation through production of $\mathrm{Ca}^{2+}$ dependent vasoactive substances such as $\mathrm{NO}$ and endothelial derived hyperpolarizing factors (EDHF). Myoendothelial projections (i.e. extension of EC which reach towards the SMC side and allow communication between the EC and SMC) are reported to retain enriched expression of a variety of channels, proteins, receptors and molecules. In the presence of the enriched expressions of cellular components, localized sub-cellular $\mathrm{Ca}^{2+}$ signaling in these spatially restricted sites is suggested to be critical in the regulation of vessel tone.

\subsection{Theoretical Models}

In an attempt to unravel, quantify and reconcile certain aspects of spatiotemporal $\mathrm{Ca}^{2+}$ signaling and its regulation of vessel tone, we developed detailed mathematical models of individual cells (EC and SMC), coupled cells (EC-SMC) and a multi-cellular vessel segment (layer of ECs coupled to one or more layer of SMCs). Lumped/compartmental models (i.e. models which assume uniform and well mixed distribution of cellular components in individual cells), which lack spatial resolution, were utilized for the 
examination of synchronization of global whole cell $\mathrm{Ca}^{2+}$ and $V_{\mathrm{m}}$ oscillations. Continuum models using finite element techniques (which are computationally expensive) were developed to incorporate spatial heterogeneity of cellular components and examine localized sub-cellular $\mathrm{Ca}^{2+}$ events. The total whole cell currents through individual channels and receptors were modeled using deterministic descriptions, whereas opening of a single/cluster of these channels was simulated using stochastic MARKOV chains. Theoretical investigations using the developed multi-scale models can assist in the experimental analysis, and provide quantification and elucidation of the complex regulatory mechanism underlying the spatiotemporal $\mathrm{Ca}^{2+}$ patterns observed in the vascular cells. This will lead to a better understanding of the mechanisms that regulate vascular resistance, blood flow and pressure in health and hypertension. Identifying critical signaling pathways that regulate vessel tone will provide targeted therapeutic strategies for diseases such as hypertension.

\subsection{Outcomes}

\subsubsection{EC TRPV4 mediated subcellular $\mathrm{Ca}^{2+}$ increase}

Endothelium derived hyperpolarization $(\mathrm{EDH})$ is a major mechanism for the regulation of vascular diameter in small-resistance vessels. Localized $\mathrm{Ca}^{2+}$ increase via activation of as few as three TRPV4 channels per EC have been suggested to induce maximal vessel dilations (through the generation of EDH). Random/stochastic openings of a TRPV4 channel cluster, implemented using stochastic Markov chains, resulted in a localized quantal $\mathrm{Ca}^{2+}$ increase (in a region $7-46 \mu \mathrm{m}^{2}$ in the vicinity of the cluster location site), consistent with experimental data. The model quantifies a 4-16 $\mu \mathrm{M}$ localized $\mathrm{Ca}^{2+}$ increase at the site of the channel cluster that spreads $2-7 \mu \mathrm{m}$ (radial 
distances) along the EC. The model predicts that a localized $\mathrm{Ca}^{2+}$ increase via a cluster of TRPV4 channels can result in 4-7 mV EC hyperpolarization in the model when 25-30 $\mathrm{IK}_{\mathrm{Ca}}$ channels are placed in close proximity of the TRPV4 channel cluster. The endothelial hyperpolarization can spread to adjacent SMCs to result in vessel dilation. Moreover, the model predicted a 2-4 fold increase in the $\mathrm{Ca}^{2+}$ spread arising from bursting activity and cooperative gating kinetics of TRPV4s. However, the model suggests that the amplified $\mathrm{Ca}^{2+}$ response from the burst and cooperative gating kinetics might be counterproductive and can lead to diminished hyperpolarization (if most of the $\mathrm{K}_{\mathrm{Ca}}$ channels are present adjacent to the TRPV4 cluster). The model predicts that amplification of EDH signaling by cooperativity in the TRPV4 cluster is more likely to occur if the $\mathrm{K}_{\mathrm{Ca}}$ channels are not located immediately adjacent to the TRPV4 channels, but at distances that can reach 10 microns away. Further experimentation needs to be carried out to examine the exact distribution of these channels in the vascular cells.

\subsubsection{NO feedback response}

$\mathrm{NO}$ is a potent vasodilator. Cytosolic $\mathrm{Ca}^{2+}$ levels in ECs regulate NO production. NO is significantly scavenged by Hemoglobin $(\mathrm{Hb})$ in the red blood cells (RBCs). SMC stimulation has been suggested to cause an $\mathrm{IP}_{3}$-mediated $\mathrm{Ca}^{2+}$ transient in the MPs with limited global spread in the bulk EC. Continuum model of coupled EC and SMC (ECMP-SMC), incorporated with NO-cGMP pathway, suggested the necessity of spatially localized eNOS in the MP for significant NO production during SMC stimulation. This was a consequence of limited bulk $\mathrm{EC} \mathrm{Ca}^{2+}$ increases during SMC stimulations. In vitro experimental studies (in the absence of blood) have shown presence of $\mathrm{Hb} \alpha$ (a scavenger of $\mathrm{NO}$ ) in the $\mathrm{EC}$ and suggested its role as switch regulating $\mathrm{NO}$ signaling. In the absence 
of RBCs, $0.05-0.1 \mu \mathrm{M} \mathrm{Hb} \alpha$ localized in the MP resulted in diminished NO feedback response, consistent with the experimental reports. However, the simulations suggested that $\mathrm{RBC}$ perfusion will decrease the ability of $\mathrm{EC} \mathrm{Hb} \alpha$ to modulate NO levels, and high $\mu \mathrm{M}$ (around $0.5-1 \mu \mathrm{M})$ levels of EC Hb $\alpha$ (10-100 fold higher than observed eNOS concentration) were necessary for a significant modulation of SMC NO availability. Further experimentation quantifying the $\mathrm{EC} \mathrm{Hb} \alpha$ concentration will aid in better understanding the role of spatially localized EC Hba in regulation of the NO mediated vasodilatory feedback response.

\subsubsection{Oscillatory intracellular $\mathrm{Ca}^{2+}$ waves in $\mathrm{EC}$ and SMC}

In addition to the subcellular localized $\mathrm{Ca}^{2+}$ events, agonist stimulated repetitive intracellular $\mathrm{Ca}^{2+}$ wave like activity in the EC and SMC is consistently observed in the experiments and are suggested to play a role in regulation of vascular tone. Moreover, a wide range in velocities $(1-100 \mu \mathrm{m} / \mathrm{s})$ for the intracellular $\mathrm{Ca}^{2+}$ waves have been reported. Continuum 1-D and 2-D models of the EC and SMC were developed and oscillatory wave like activity was reproduced, if a gradient in store (i.e. inositol triphosphate, ryanodine) or membrane (i.e. adrenergic, muscarinic) receptor density was assumed. Diffusion of $\mathrm{Ca}^{2+}$ and/or $\mathrm{IP}_{3}$ provided a weak propagating signal to synchronize neighboring domains, to result in the appearance of the propagating $\mathrm{Ca}^{2+}$ waves. Model predicts that $V_{\mathrm{m}}$ can affect wave velocity through modulation of transmembrane calcium influx and by altering the electrochemical gradient for calcium influx.

\subsection{4 $\mathrm{Ca}^{2+}$ synchronization in arterial vasomotion}

Rhythmic oscillations in vessel diameter (vasomotion) is observed in variety of vascular beds and suggested to arise from intercellular $\mathrm{Ca}^{2+}$ synchronization in individual 
SMCs, and can be influenced by the endothelium. The phenomenon of vasomotion is thought to improve tissue oxygenation and enhance blood flow. A multi-cellular model of coupled SMCs with ECs to form a vessel segment with individual SMCs oscillating at varied intrinsic frequencies was developed and the synchronization mechanism were examined. Model simulations predicts several physiologically feasible pathways for synchronization of $\mathrm{Ca}^{2+}$ oscillations. These include depolarizing current generated through $\mathrm{Cl}_{\mathrm{Ca}}$ and NSC channels, or pathways that involve the intercellular diffusion of $\mathrm{IP}_{3}$ and perhaps of $\mathrm{Ca}^{2+}$.

$\mathrm{Ca}^{2+}$ events in the vascular cells occur at varied spatial and temporal scales. Isolated or repetitive $\mathrm{Ca}^{2+}$ transients lasting for few milliseconds to $\mathrm{Ca}^{2+}$ events with half-life in the order of seconds are observed occurring randomly or in persistent regions of the vascular cells. The $\mathrm{Ca}^{2+}$ events are either confined to small regions or appear to propagate across or in between cells. The developed models capture such events and provide insights for their physiological relevance. Further research is required to elucidate and better comprehend the complex spatio-temporal nature of $\mathrm{Ca}^{2+}$ signaling. This will allow us gaining a deeper understanding of vascular tone regulation in health and disease. 
CHAPTER 7-APPENDIX 


\subsection{Model Equations}

\subsubsection{Intercellular coupling}

7.1.1.1 Ionic coupling

$$
\begin{gathered}
I_{\mathrm{gj}}=\sum_{\mathrm{S}} I_{\mathrm{gj}, \mathrm{S}} \\
I_{\mathrm{gj}, \mathrm{S}}=P_{\mathrm{S}} z_{\mathrm{S}}^{2} \frac{V_{\mathrm{gj}} F^{2}}{R T} \frac{[\mathrm{S}]_{\mathrm{i}}^{n}-[\mathrm{S}]_{\mathrm{i}}^{m} \exp \left(-z_{\mathrm{S}} V_{\mathrm{gj}} F / R T\right)}{1-\exp \left(-z_{\mathrm{S}} V_{\mathrm{gj}} F / R T\right)} \\
P_{\mathrm{S}}=\frac{R T}{F^{2} R_{\mathrm{gj}} \sum_{\mathrm{S}}\left(z_{\mathrm{S}}^{2}[\mathrm{~S}]_{\mathrm{i}}\right)} \\
\mathrm{S}=\mathrm{Ca}^{2+}, \mathrm{K}^{+}, \mathrm{Na}^{+}, \mathrm{Cl}^{-} ; V_{\mathrm{gj}}=V_{\mathrm{m}}^{n}-V_{\mathrm{m}}^{m}, n, m-\text { cell index; } \\
R_{\mathrm{gj}}-\text { gap junction resistance. }
\end{gathered}
$$

$\mathrm{z}_{\mathrm{K}}=\mathrm{z}_{\mathrm{Na}}=1 ; \mathrm{z}_{\mathrm{Ca}}=2 ; \mathrm{z}_{\mathrm{Cl}}=-1 ; \mathrm{N}_{\mathrm{Av}}=6.022 \times 10^{23} ; R=8314 \mathrm{~mJ} / \mathrm{mol} \cdot \mathrm{K} ; F=96485 \mathrm{C} / \mathrm{mol} ;$ $T=293.0 \mathrm{~K} ;[\mathrm{Ca}]_{\mathrm{o}}=2.0 \mathrm{mM} ;[\mathrm{Na}]_{\mathrm{o}}=140.0 \mathrm{mM} ;[\mathrm{Cl}]_{\mathrm{o}}=129.0 \mathrm{mM} ;[\mathrm{K}]_{\mathrm{o}}=5.0 \mathrm{mM}$.

\subsubsection{IP 3 coupling}

$$
J_{\mathrm{IP} 3}=P_{\mathrm{IP} 3}\left(\left[\mathrm{IP}_{3}\right]^{n}-\left[\mathrm{IP}_{3}\right]^{m}\right)
$$

7.1.1.3 NO coupling

$$
\begin{gathered}
\bar{Q}_{N O, s s}^{E C}=\frac{Q_{N O, s S}^{E C}}{Q_{N O, \text { max }}^{E C}}=\frac{\left(\left[\mathrm{Ca}^{2+}\right]_{\mathrm{i}}^{\mathrm{EC}}\right)^{n}}{\left(\left[\mathrm{Ca}^{2+}\right]_{\mathrm{i}}^{\mathrm{EC}}\right)^{n}+\left(K_{\mathrm{m}, \mathrm{Ca}}^{\mathrm{NO}}\right)^{n}} \\
\frac{d \bar{Q}_{N O}^{E C}}{d t}=\frac{\bar{Q}_{N O, s s}^{E C}-\bar{Q}_{N O}^{E C}}{\tau_{\mathrm{eNOS}}} \\
{[\mathrm{NO}]^{\mathrm{SM}}=[\mathrm{NO}]_{\max } \bar{Q}_{N O}^{E C}} \\
\tau_{\mathrm{eNOS}}=2 \mathrm{~s} ; K_{\mathrm{m}, \mathrm{Ca}}^{\mathrm{NO}}=300 \mathrm{nM} ; n=4.2 ;[\mathrm{NO}]_{\max }=380 \mathrm{nM} .
\end{gathered}
$$




\subsubsection{Smooth muscle cells}

\subsubsection{Membrane electrophysiology}

$$
\begin{gathered}
\frac{\mathrm{d} V_{\mathrm{m}}}{\mathrm{d} t}=\frac{-\left(I_{\mathrm{VOCC}}+I_{\mathrm{Kv}}+I_{\mathrm{BKCa}}+I_{\mathrm{Kleak}}+I_{\mathrm{NSC}}+I_{\mathrm{SOC}}+I_{\mathrm{CICa}}+I_{\mathrm{PMCA}}+I_{\mathrm{NaK}}+I_{\mathrm{NCX}}+\sum I_{\mathrm{gj}}\right)+I_{\mathrm{stim}}}{\mathrm{C}_{\mathrm{m}}} \\
\mathrm{C}_{\mathrm{m}}=25 \mathrm{pF} .
\end{gathered}
$$

7.1.2.1.1 L-type voltage-operated $\mathrm{Ca}^{2+}$ channels

$$
\begin{gathered}
I_{\mathrm{VOCC}}=\mathrm{A}_{\mathrm{m}} \mathrm{P}_{\mathrm{VOCC}} d_{\mathrm{L}} f_{\mathrm{L}} V_{\mathrm{m}} \frac{\left(\mathrm{z}_{\mathrm{Ca}} \mathrm{F}\right)^{2}}{\mathrm{RT}} \frac{[\mathrm{Ca}]_{\mathrm{o}}-[\mathrm{Ca}]_{\mathrm{i}} \exp \left(\frac{V_{\mathrm{m}} \mathrm{z}_{\mathrm{CF}} \mathrm{F}}{\mathrm{RT}}\right)}{1-\exp \left(\frac{V_{\mathrm{m}} \mathrm{z}_{\mathrm{Ca}} \mathrm{F}}{\mathrm{RT}}\right)} \\
\frac{\mathrm{d} d_{\mathrm{L}}}{\mathrm{d} t}=\frac{\bar{d}_{\mathrm{L}}-d_{\mathrm{L}}}{\tau_{d \mathrm{~L}}} ; \bar{d}_{\mathrm{L}}=\left(1+\exp \left(-\frac{V_{\mathrm{m}}}{8.3 \mathrm{mV}}\right)\right)^{-1} ; \tau_{d \mathrm{~L}}=2.5 \exp \left(-\left(\frac{V_{\mathrm{m}}+40 \mathrm{mV}}{30 \mathrm{mV}}\right)^{2}\right)+1.15[\mathrm{~ms}] \\
\frac{\mathrm{d} f_{\mathrm{L}}}{\mathrm{d} t}=\frac{\bar{f}_{\mathrm{L}}-f_{\mathrm{L}}}{\tau_{f \mathrm{~L}}} ; \bar{f}_{\mathrm{L}}=\left(1+\exp \left(\frac{V_{\mathrm{m}}+42.0 \mathrm{mV}}{9.1 \mathrm{mV}}\right)\right)^{-1} ; \tau_{f \mathrm{~L}}=65 \exp \left(-\left(\frac{V_{\mathrm{m}}+35 \mathrm{mV}}{25 \mathrm{mV}}\right)^{2}\right)+45[\mathrm{~ms}] \\
\mathrm{P}_{\mathrm{VOCC}}=1.88 \times 10^{-5} \mathrm{~cm} / \mathrm{s} ; \mathrm{A}_{\mathrm{m}}=\mathrm{C}_{\mathrm{m}}\left(10^{-6} \mathrm{~cm}^{2} / \mathrm{pF}\right) .
\end{gathered}
$$

7.1.2.1.2 Large conductance $\mathrm{Ca}^{2+}$-activated $\mathrm{K}^{+}$channels

$$
\begin{gathered}
I_{\mathrm{BKCa}}=\mathrm{A}_{\mathrm{m}} \mathrm{N}_{\mathrm{BKCa}} P_{\mathrm{KCa}} i_{\mathrm{KCa}} \\
P_{\mathrm{KCa}}=0.17 p_{\mathrm{f}}+0.83 p_{\mathrm{s}} ; \frac{\mathrm{d} p_{\mathrm{f}}}{\mathrm{d} t}=\frac{\bar{p}_{\mathrm{o}}-p_{\mathrm{f}}}{\tau_{p \mathrm{f}}} ; \frac{\mathrm{d} p_{\mathrm{s}}}{\mathrm{d} t}=\frac{\bar{p}_{\mathrm{o}}-p_{\mathrm{s}}}{\tau_{p \mathrm{~s}}} \\
\bar{p}_{\mathrm{o}}=\left(1+\exp \left(-\frac{V_{\mathrm{m}}-V_{1 / 2 \mathrm{KCa}}}{18.25 \mathrm{mV}}\right)\right)^{-1} \\
V_{1 / 2 \mathrm{KCa}}=(-41.7 \mathrm{mV}) \log _{10}\left([\mathrm{Ca}]_{\mathrm{i}} /(1 \mathrm{mM})\right)-128.2 \mathrm{mV}-\mathrm{dV}_{1 / 2 \mathrm{KCaNO}} R_{\mathrm{NO}}-\mathrm{dV}_{1 / 2 \mathrm{KCaCGMP}} R_{\mathrm{cGMP}} \\
R_{\mathrm{NO}}=\frac{[\mathrm{NO}]}{[\mathrm{NO}]+200 \mathrm{nM}} ; R_{\mathrm{cGMP}}=\frac{[\mathrm{cGMP}]^{2}}{[\mathrm{cGMP}]^{2}+(1.5 \mu \mathrm{M})^{2}}
\end{gathered}
$$




$$
i_{\mathrm{KCa}}=\mathrm{P}_{\mathrm{BKCa}} V_{\mathrm{m}} \frac{\mathrm{F}^{2}}{\mathrm{RT}} \frac{[\mathrm{K}]_{\mathrm{o}}-[\mathrm{K}]_{\mathrm{i}} \exp \left(\frac{V_{\mathrm{m}} \mathrm{F}}{\mathrm{RT}}\right)}{1-\exp \left(\frac{V_{\mathrm{m}} \mathrm{F}}{\mathrm{RT}}\right)}
$$

$\mathrm{P}_{\mathrm{BKCa}}=3.9 \times 10^{-13} \mathrm{~cm}^{3} / \mathrm{s} ; \mathrm{dV}_{1 / 2 \mathrm{KCaNO}}=46.3 \mathrm{mV} ; \mathrm{N}_{\mathrm{BKCa}}=6.6 \times 10^{6} \mathrm{~cm}^{-2} ; \tau_{p \mathrm{f}}=0.84 \mathrm{~ms} ; \tau_{p \mathrm{~s}}$

$$
\begin{gathered}
=35.9 \mathrm{~ms} \\
\mathrm{dV}_{1 / 2 \mathrm{KCacGMP}}=76 \mathrm{mV}
\end{gathered}
$$

7.1.2.1.3 Voltage-dependent $\mathrm{K}^{+}$channels

$$
\begin{gathered}
I_{\mathrm{Kv}}=\mathrm{g}_{\mathrm{Kv}} p_{\mathrm{K}}\left(0.45 q_{1}+0.55 q_{2}\right)\left(V_{\mathrm{m}}-E_{\mathrm{K}}\right) \\
\bar{p}_{\mathrm{K}}=\frac{1}{1+\exp \left(-\frac{V_{\mathrm{m}}+11.0 \mathrm{mV}}{15.0 \mathrm{mV}}\right)} ; \tau_{p \mathrm{~K}}=61.5 \exp \left(-0.027 V_{\mathrm{m}}\right)[\mathrm{ms}] ; \frac{\mathrm{d} p_{\mathrm{K}}}{\mathrm{d} t}=\frac{\bar{p}_{\mathrm{K}}-p_{\mathrm{K}}}{\tau_{p \mathrm{~K}}} \\
\bar{q}=\frac{1}{1+\exp \left(\frac{V_{\mathrm{m}}+40.0 \mathrm{mV}}{14.0 \mathrm{mV}}\right)} ; \frac{\mathrm{d} q_{1}}{\mathrm{~d} t}=\frac{\bar{q}-q_{1}}{\tau_{q 1}} ; \frac{\mathrm{d} q_{2}}{\mathrm{~d} t}=\frac{\bar{q}-q_{2}}{\tau_{q 2}} \\
\mathrm{~g}_{\mathrm{Kv}}=1.35 \mathrm{nS} ; \tau_{q 1}=371 \mathrm{~ms} ; \tau_{q 2}=2884 \mathrm{~ms} .
\end{gathered}
$$

7.1.2.1.4 Unspecified $\mathrm{K}^{+}$leak channels

$$
\begin{gathered}
I_{\text {Kleak }}=\mathrm{g}_{\text {Kleak }}\left(V_{\mathrm{m}}-E_{\mathrm{K}}\right) \\
\mathrm{g}_{\text {Kleak }}=0.067 \mathrm{nS} .
\end{gathered}
$$

7.1.2.1.5 Nonselective cation channels

$$
I_{\mathrm{NSC}}=I_{\mathrm{NaNSC}}+I_{\mathrm{KNSC}}+I_{\mathrm{CaNSC}}
$$




$$
\begin{gathered}
I_{\mathrm{NaNSC}}=\mathrm{A}_{\mathrm{m}}\left(\frac{[\mathrm{DAG}]}{[\mathrm{DAG}]+\mathrm{K}_{\mathrm{NSC}}}+\mathrm{d}_{\mathrm{NSCmin}}\right) P_{\mathrm{ONSC}} \mathrm{P}_{\mathrm{NaNSC}} V_{\mathrm{m}} \frac{\mathrm{F}^{2}}{\mathrm{RT}} \frac{[\mathrm{Na}]_{\mathrm{o}}-[\mathrm{Na}]_{\mathrm{i}} \exp \left(\frac{V_{\mathrm{m}} \mathrm{F}}{\mathrm{RT}}\right)}{1-\exp \left(\frac{V_{\mathrm{m}} \mathrm{F}}{\mathrm{RT}}\right)} \\
I_{\mathrm{KNSC}}=\mathrm{A}_{\mathrm{m}}\left(\frac{[\mathrm{DAG}]}{[\mathrm{DAG}]+\mathrm{K}_{\mathrm{NSC}}}+\mathrm{d}_{\mathrm{NSCmin}}\right) P_{\mathrm{oNSC}} \mathrm{P}_{\mathrm{KNSC}} V_{\mathrm{m}} \frac{\mathrm{F}^{2}}{\mathrm{RT}} \frac{[\mathrm{K}]_{\mathrm{o}}-[\mathrm{K}]_{\mathrm{i}} \exp \left(\frac{V_{\mathrm{m}} \mathrm{F}}{\mathrm{RT}}\right)}{1-\exp \left(\frac{V_{\mathrm{m}} \mathrm{F}}{\mathrm{RT}}\right)} \\
I_{\mathrm{CaNSC}}=\mathrm{A}_{\mathrm{m}} \mathrm{d}_{\mathrm{NSCmin}} P_{\mathrm{oNSC}} \mathrm{P}_{\mathrm{CaNSC}} V_{\mathrm{m}} \frac{\left(\mathrm{z}_{\mathrm{Ca}} \mathrm{F}\right)^{2}}{\mathrm{RT}} \frac{[\mathrm{Ca}]_{\mathrm{o}}-[\mathrm{Ca}]_{\mathrm{i}} \exp \left(\frac{\mathrm{z}_{\mathrm{Ca}} V_{\mathrm{m}} \mathrm{F}}{\mathrm{RT}}\right)}{1-\exp \left(\frac{\mathrm{z}_{\mathrm{Ca}} V_{\mathrm{m}} \mathrm{F}}{\mathrm{RT}}\right)} \\
P_{\mathrm{oNSC}}=0.43+\frac{0.57}{1+\exp \left(-\frac{V_{\mathrm{m}}-47.12 \mathrm{mV}}{24.24 \mathrm{mV}}\right)}
\end{gathered}
$$

$\mathrm{K}_{\mathrm{NSC}}=3 \mu \mathrm{M} ; \mathrm{P}_{\mathrm{NaNSC}}=5.11 \times 10^{-7} \mathrm{~cm} / \mathrm{s} ; \mathrm{P}_{\mathrm{KNSC}}=1.06 \times \mathrm{P}_{\mathrm{NaNSC}} ; \mathrm{P}_{\mathrm{CaNSC}}=4.54 \times \mathrm{P}_{\mathrm{NaNSC}} ;$

$$
\mathrm{d}_{\mathrm{NSCmin}}=0.0244
$$

7.1.2.1.6 Store-operated nonselective cation channels

$$
\begin{gathered}
I_{\mathrm{SOC}}=I_{\mathrm{SOCCa}}+I_{\mathrm{SOCNa}} \\
I_{\mathrm{SOCCa}}=\mathrm{g}_{\mathrm{SOCCa}} P_{\mathrm{SOC}}\left(V_{\mathrm{m}}-E_{\mathrm{Ca}}\right) ; I_{\mathrm{SOCNa}}=\mathrm{g}_{\mathrm{SOCNa}} P_{\mathrm{SOC}}\left(V_{\mathrm{m}}-E_{\mathrm{Na}}\right) \\
P_{\mathrm{SOC}}=\left(1+\frac{[\mathrm{Ca}]_{\mathrm{u}}}{100 \mathrm{nM}}\right)^{-1} \\
\mathrm{~g}_{\mathrm{SOCCa}}=0.0083 \mathrm{nM} ; \mathrm{g}_{\mathrm{SOCNa}}=0.0575 \mathrm{nM} .
\end{gathered}
$$

7.1.2.1.7 Calcium-activated chloride channels

$$
I_{\mathrm{ClCa}}=\mathrm{C}_{\mathrm{m}} \mathrm{g}_{\mathrm{ClCa}} P_{\mathrm{Cl}}\left(V_{\mathrm{m}}-E_{\mathrm{Cl}}\right)
$$




$$
\begin{gathered}
P_{\mathrm{Cl}}=0.0132 \frac{\left([\mathrm{Ca}]_{\mathrm{i}}\right)^{\mathrm{n}_{\mathrm{ClCa}}}}{\left([\mathrm{Ca}]_{\mathrm{i}}\right)^{\mathrm{n}_{\mathrm{ClCa}}}+\left(\mathrm{K}_{\mathrm{ClCa}}\right)^{\mathrm{n}_{\mathrm{ClCa}}}}+\alpha_{\mathrm{Cl}} \frac{\left([\mathrm{Ca}]_{\mathrm{i}}\right)^{\mathrm{n}_{\mathrm{ClCa}}}}{\left([\mathrm{Ca}]_{\mathrm{i}}\right)^{\mathrm{n}_{\mathrm{ClCa}}}+\left(\mathrm{K}_{\mathrm{ClCa}, \mathrm{CGMP}}\right)^{\mathrm{n}_{\mathrm{ClCa}}}} \\
\alpha_{\mathrm{Cl}}=\frac{([\mathrm{cGMP}])^{\mathrm{n}_{\mathrm{ClcGMP}}}}{([\mathrm{cGMP}])^{\mathrm{n}_{\mathrm{ClCGMP}}}+\left(\mathrm{K}_{\mathrm{ClcGMP}}\right)^{\mathrm{n}_{\mathrm{ClcGMP}}}} \mathrm{K}_{\mathrm{ClCa}, \mathrm{cGMP}}=\left(1-0.9 \alpha_{\mathrm{Cl}}\right) \cdot 400 \mathrm{nM} \\
\mathrm{g}_{\mathrm{ClCa}}=0.23 \mathrm{nS} / \mathrm{pF} ; \mathrm{n}_{\mathrm{ClCa}}=2 ; \mathrm{K}_{\mathrm{ClCa}}=365 \mathrm{nM} ; \mathrm{n}_{\mathrm{ClcGMP}}=3.3 ; \mathrm{K}_{\mathrm{ClcGMP}}=6.4 \mu \mathrm{M} .
\end{gathered}
$$

7.1.2.1.8 Plasma membrane $\mathrm{Ca}^{2+}$ pump

$$
\begin{gathered}
I_{\mathrm{PMCA}}=\overline{\mathrm{I}}_{\mathrm{PMCA}} \frac{[\mathrm{Ca}]_{\mathrm{i}}}{[\mathrm{Ca}]_{\mathrm{i}}+\mathrm{K}_{\mathrm{m}, \mathrm{PMCA}}} \\
\overline{\mathrm{I}}_{\mathrm{PMCA}}=5.37 \mathrm{pA} ; \mathrm{K}_{\mathrm{m}, \mathrm{PMCA}}=170 \mathrm{nM} .
\end{gathered}
$$

7.1.2.1.9 Plasma membrane $\mathrm{Na}^{+}-\mathrm{Ca}^{2+}$ exchange

$$
\begin{gathered}
I_{\mathrm{NCX}}=\mathrm{g}_{\mathrm{NCX}} R_{\mathrm{NCX}, \mathrm{cGMP}} \frac{[\mathrm{Na}]_{\mathrm{i}}^{3}[\mathrm{Ca}]_{\mathrm{o}} \Phi_{\mathrm{F}}-[\mathrm{Na}]_{\mathrm{o}}^{3}[\mathrm{Ca}]_{\mathrm{i}} \Phi_{\mathrm{R}}}{(1 \mathrm{mM})^{4}+\mathrm{d}_{\mathrm{NCX}}\left([\mathrm{Na}]_{\mathrm{o}}^{3}[\mathrm{Ca}]_{\mathrm{i}}+[\mathrm{Na}]_{\mathrm{i}}^{3}[\mathrm{Ca}]_{\mathrm{o}}\right)} \\
R_{\mathrm{NCX}, \mathrm{cGMP}}=1+0.55 \frac{[\mathrm{cGMP}]}{[\mathrm{cGMP}]+45 \mu \mathrm{M}} \\
\Phi_{\mathrm{F}}=\exp \left(\frac{\gamma V_{\mathrm{m}} \mathrm{F}}{\mathrm{RT}}\right) ; \Phi_{\mathrm{R}}=\exp \left(\frac{(\gamma-1) V_{\mathrm{m}} \mathrm{F}}{\mathrm{RT}}\right) ; \gamma=0.45 ; \mathrm{d}_{\mathrm{NCX}}=0.0003 ; \mathrm{g}_{\mathrm{NCX}}=4.87 \times 10^{-4} \mathrm{pA}
\end{gathered}
$$

\subsection{Sodium-potassium pump}

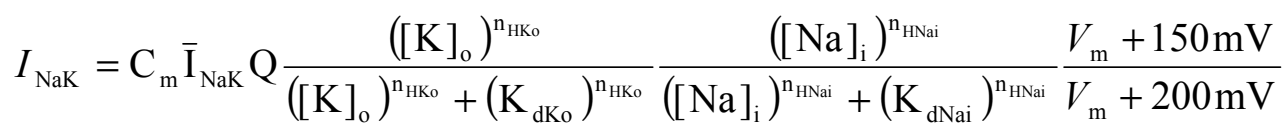

$$
\begin{aligned}
& \mathrm{Q}=\mathrm{Q}_{10} \frac{\mathrm{T}-309.15 \mathrm{~K}}{10} ; \mathrm{Q}_{10}=1.87 \\
& \overline{\mathrm{I}}_{\mathrm{NaK}}=2.31 \mathrm{pA} / \mathrm{pF} ; \mathrm{K}_{\mathrm{dKo}}=1.6 \mathrm{mM} ; \mathrm{K}_{\mathrm{dNai}}=22 \mathrm{mM} ; \mathrm{n}_{\mathrm{HKo}}=1.1 ; \mathrm{n}_{\mathrm{HNai}}=1.7 \text {. }
\end{aligned}
$$

\subsection{Sodium-potassium-chloride cotransport}

$$
I_{\mathrm{NaKCl}}^{\mathrm{Cl}}=-R_{\mathrm{NaKCl,cGMP}} z_{\mathrm{Cl}} \mathrm{A}_{\mathrm{m}} \mathrm{L}_{\mathrm{NaKCl}} \mathrm{RFT} \log _{\mathrm{e}}\left(\frac{[\mathrm{Na}]_{\mathrm{o}}}{[\mathrm{Na}]_{\mathrm{i}}} \frac{[\mathrm{K}]_{\mathrm{o}}}{[\mathrm{K}]_{\mathrm{i}}}\left(\frac{[\mathrm{Cl}]_{\mathrm{o}}}{[\mathrm{Cl}]_{\mathrm{i}}}\right)^{2}\right)
$$




$$
\begin{gathered}
R_{\mathrm{NaKCl}, \mathrm{GGMP}}=1+3.5 \frac{[\mathrm{cGMP}]}{[\mathrm{cGMP}]+6.4 \mu \mathrm{M}} \\
I_{\mathrm{NaKCl}}^{\mathrm{Na}}=I_{\mathrm{NaKCl}}^{\mathrm{K}}=-0.5 I_{\mathrm{NaKCl}}^{\mathrm{Cl}} \\
\mathrm{L}_{\mathrm{NaKCl}}=1.79 \times 10^{-11}(\mathrm{mmol})^{2} /\left(\mathrm{J} \cdot \mathrm{s} \cdot \mathrm{cm}^{2}\right) .
\end{gathered}
$$

Reversal potentials

$E_{\mathrm{A}}=\frac{\mathrm{RT}}{\mathrm{z}_{\mathrm{A}} \mathrm{F}} \ln \left(\frac{[\mathrm{A}]_{\mathrm{o}}}{[\mathrm{A}]_{\mathrm{i}}}\right)$, where A denotes $\mathrm{K}, \mathrm{Na}, \mathrm{Ca}$ or $\mathrm{Cl}$.

\subsubsection{Sarcoplasmic reticulum}

$$
\begin{aligned}
& I_{\text {SERCA }}=\bar{I}_{\text {SERCA }} \frac{[\mathrm{Ca}]_{\mathrm{i}}}{[\mathrm{Ca}]_{\mathrm{i}}+\mathrm{K}_{\mathrm{m}, \text { up }}} \\
& I_{\mathrm{tr}}=\left([\mathrm{Ca}]_{\mathrm{u}}-[\mathrm{Ca}]_{\mathrm{r}}\right) \mathrm{z}_{\mathrm{Ca}} \mathrm{Fvol}_{\mathrm{u}} / \tau_{\mathrm{tr}} \\
& I_{\text {rel }}=\left(R_{10}^{2}+\mathrm{R}_{\text {leak }}\right)\left([\mathrm{Ca}]_{\mathrm{r}}-[\mathrm{Ca}]_{\mathrm{i}}\right) \mathrm{z}_{\mathrm{Ca}} \mathrm{Fvol}_{\mathrm{r}} / \tau_{\text {rel }} \\
& \frac{\mathrm{d}[\mathrm{Ca}]_{\mathrm{u}}}{\mathrm{d} t}=\frac{I_{\mathrm{SERCA}}-I_{\mathrm{tr}}-I_{\mathrm{IP} 3}}{\mathrm{z}_{\mathrm{Ca}} \mathrm{Fvol}_{\mathrm{u}}} \\
& \frac{\mathrm{d}[\mathrm{Ca}]_{\mathrm{r}}}{\mathrm{d} t}=\frac{I_{\mathrm{tr}}-I_{\mathrm{rel}}}{\mathrm{z}_{\mathrm{Ca}} \mathrm{Fvol}_{\mathrm{r}}}\left(1+\frac{[\overline{\mathrm{CSQN}}] \mathrm{K}_{\mathrm{CSQN}}}{\left(\mathrm{K}_{\mathrm{CSQN}}+[\mathrm{Ca}]_{\mathrm{r}}\right)^{2}}\right)^{-1} \\
& \tau_{\mathrm{tr}}=1000 \mathrm{~ms} ; \tau_{\text {rel }}=0.033 \mathrm{~ms} \\
& \mathrm{~K}_{\mathrm{m}, \mathrm{up}}=1 \mu \mathrm{M} ; \bar{I}_{\mathrm{SERCA}}=20.04 \mathrm{pA} ; R_{\text {leak }}=5.35 \times 10^{-5} ; \mathrm{K}_{\mathrm{CSQN}}=0.8 \mathrm{mM} ;[\overline{\mathrm{CSQN}}]=15 \\
& \mathrm{mM} ; \operatorname{vol}_{\mathrm{u}}=0.07 \mathrm{pL} ; \\
& \mathrm{vol}_{\mathrm{r}}=0.007 \mathrm{pL} .
\end{aligned}
$$


7.1.2.2.1 Ryanodine receptor

$$
\begin{gathered}
R_{00}=1-R_{01}-R_{10}-R_{11} \\
\frac{\mathrm{d} R_{10}}{\mathrm{~d} t}=\mathrm{K}_{\mathrm{r} 1}[\mathrm{Ca}]_{\mathrm{i}}^{2} R_{00}-\left(\mathrm{K}_{-\mathrm{r} 1}+\mathrm{K}_{\mathrm{r} 2}[\mathrm{Ca}]_{\mathrm{i}}\right) R_{10}+\mathrm{K}_{-\mathrm{r} 2} R_{11} \\
\frac{\mathrm{d} R_{11}}{\mathrm{~d} t}=\mathrm{K}_{\mathrm{r} 2}[\mathrm{Ca}]_{\mathrm{i}} R_{10}-\left(\mathrm{K}_{-\mathrm{r} 1}+\mathrm{K}_{-\mathrm{r} 2}\right) R_{11}+\mathrm{K}_{\mathrm{r} 1}[\mathrm{Ca}]_{\mathrm{i}}^{2} R_{01} \\
\frac{\mathrm{d} R_{01}}{\mathrm{~d} t}=\mathrm{K}_{\mathrm{r} 2}[\mathrm{Ca}]_{\mathrm{i}} R_{00}+\mathrm{K}_{-\mathrm{r} 1} R_{11}-\left(\mathrm{K}_{-\mathrm{r} 2}+\mathrm{K}_{\mathrm{r} 1}[\mathrm{Ca}]_{\mathrm{i}}^{2}\right) R_{01} \\
\mathrm{~K}_{\mathrm{r} 1}=2500 \mathrm{mM}^{-2} \mathrm{~ms}^{-1} ; \mathrm{K}_{\mathrm{r} 2}=1.05 \mathrm{mM}^{-1} \mathrm{~ms}^{-1} ; \mathrm{K}_{-\mathrm{r} 1}=0.0076 \mathrm{~ms}^{-1} ; \mathrm{K}_{-\mathrm{r} 2}=0.084 \mathrm{~ms}^{-1}
\end{gathered}
$$

\subsubsection{2 $\mathrm{IP}_{3}$ receptor}

$$
\begin{aligned}
& I_{\mathrm{IP} 3}=\overline{\mathrm{I}}_{\mathrm{IP3}} \mathrm{z}_{\mathrm{Ca}} \mathrm{vol}_{\mathrm{Ca}} \mathrm{F}\left(\frac{\left[\mathrm{IP}_{3}\right]}{\left[\mathrm{IP}_{3}\right]+\mathrm{K}_{\mathrm{IP} 3}} \frac{[\mathrm{Ca}]_{\mathrm{i}}}{[\mathrm{Ca}]_{\mathrm{i}}+\mathrm{K}_{\mathrm{ac}, \mathrm{IP} 3}} h_{\mathrm{IP} 3}\right)^{3}\left([\mathrm{Ca}]_{\mathrm{u}}-[\mathrm{Ca}]_{\mathrm{i}}\right) \\
& \frac{\mathrm{d} h_{\mathrm{IP} 3}}{\mathrm{~d} t}=\mathrm{k}_{\mathrm{on}, \mathrm{IP} 3}\left(\mathrm{~K}_{\mathrm{inh}, \mathrm{IP} 3}-\left([\mathrm{Ca}]_{\mathrm{i}}+\mathrm{K}_{\mathrm{inh}, \mathrm{IP} 3}\right) h_{\mathrm{IP} 3}\right) \\
& \overline{\mathrm{I}}_{\mathrm{IP3}}=2880 \times 10^{-6} \mathrm{~ms}^{-1} ; \mathrm{K}_{\mathrm{act}, \mathrm{IP} 3}=170 \mathrm{nM} ; \mathrm{K}_{\mathrm{inh}, \mathrm{IP3}}=100 \mathrm{nM} ; \mathrm{K}_{\mathrm{IP} 3}=120 \mathrm{nM} ; \mathrm{k}_{\mathrm{on}, \mathrm{IP} 3}=1.4 \\
& \mathrm{mM}^{-1} \mathrm{~ms}^{-1} \text {. }
\end{aligned}
$$

\subsubsection{3 $\alpha_{1}$-adrenoceptor activation and $I P_{3}$ and DAG formation}

$$
\begin{gathered}
\frac{\mathrm{d}\left[\mathrm{R}_{\mathrm{G}}^{\mathrm{S}}\right]}{\mathrm{d} t}=\mathrm{k}_{\mathrm{r}, \mathrm{G}} \xi_{\mathrm{G}}\left[\mathrm{R}_{\mathrm{T}, \mathrm{G}}\right]-\left(\mathrm{k}_{\mathrm{r}, \mathrm{G}}+\frac{\mathrm{k}_{\mathrm{p}, \mathrm{G}}[\mathrm{NE}]}{\mathrm{K}_{\mathrm{l}, \mathrm{G}}+[\mathrm{NE}]}\right)\left[\mathrm{R}_{\mathrm{G}}^{\mathrm{S}}\right]-\mathrm{k}_{\mathrm{r}, \mathrm{G}}\left[\mathrm{R}_{\mathrm{P}, \mathrm{G}}^{\mathrm{S}}\right] \\
\frac{\mathrm{d}\left[\mathrm{R}_{\mathrm{P}, \mathrm{G}}^{\mathrm{S}}\right]}{\mathrm{d} t}=[\mathrm{NE}]\left(\frac{\mathrm{k}_{\mathrm{p}, \mathrm{G}}\left[\mathrm{R}_{\mathrm{G}}^{\mathrm{S}}\right]}{\mathrm{K}_{1, \mathrm{G}}+[\mathrm{NE}]}-\frac{\mathrm{k}_{\mathrm{e}, \mathrm{G}}\left[\mathrm{R}_{\mathrm{P}, \mathrm{G}}^{\mathrm{S}}\right]}{\mathrm{K}_{2, \mathrm{G}}+[\mathrm{NE}]}\right) \\
\rho_{\mathrm{r}, \mathrm{G}}=\frac{[\mathrm{NE}]\left[\mathrm{R}_{\mathrm{G}}^{\mathrm{S}}\right]}{\xi_{\mathrm{G}}\left[\mathrm{R}_{\mathrm{T}, \mathrm{G}}\right]\left(\mathrm{K}_{\mathrm{l}, \mathrm{G}}+[\mathrm{NE}]\right)}
\end{gathered}
$$




$$
\begin{aligned}
& \frac{\mathrm{d}[\mathrm{G}]}{\mathrm{d} t}=\mathrm{k}_{\mathrm{a}, \mathrm{G}}\left(\delta_{\mathrm{G}}+\rho_{\mathrm{r}, \mathrm{G}}\right)\left(\left[\mathrm{G}_{\mathrm{T}, \mathrm{G}}\right]-[\mathrm{G}]\right)-\mathrm{k}_{\mathrm{d}, \mathrm{G}}[\mathrm{G}] \\
& r_{\mathrm{h}, \mathrm{G}}=\alpha_{\mathrm{G}} \frac{[\mathrm{Ca}]_{\mathrm{i}}}{[\mathrm{Ca}]_{\mathrm{i}}+\mathrm{K}_{\mathrm{c}, \mathrm{G}}}[\mathrm{G}] \\
& \frac{\mathrm{d}\left[\mathrm{IP}_{3}\right]}{\mathrm{d} t}=\frac{r_{\mathrm{h}, \mathrm{G}}}{\gamma_{\mathrm{G}}}\left[\mathrm{PIP}_{2}\right]-\mathrm{k}_{\mathrm{deg}, \mathrm{G}}\left[\mathrm{IP}_{3}\right]+\frac{\sum J_{\mathrm{IP} 3}}{\mathrm{vol}_{\mathrm{i}}} \\
& \frac{\mathrm{d}[\mathrm{DAG}]}{\mathrm{d} t}=\frac{r_{\mathrm{h}, \mathrm{G}}}{\gamma_{\mathrm{G}}}\left[\mathrm{PIP} \mathrm{P}_{2}\right]-\mathrm{k}_{\mathrm{deg}, \mathrm{G}}[\mathrm{DAG}] \\
& \frac{\mathrm{d}\left[\mathrm{PIP}_{2}\right]}{\mathrm{d} t}=-\left(r_{\mathrm{h}, \mathrm{G}}+\mathrm{r}_{\mathrm{r}, \mathrm{G}}\right)\left[\mathrm{PIP}_{2}\right]-\mathrm{r}_{\mathrm{r}, \mathrm{G}} \gamma_{\mathrm{G}}\left[\mathrm{IP}_{3}\right]+\mathrm{r}_{\mathrm{r}, \mathrm{G}}\left[\mathrm{PIP}_{2, \mathrm{~T}}\right] \\
& {\left[\mathrm{R}_{\mathrm{T}, \mathrm{G}}\right]=2 \times 10^{4} ; \mathrm{K}_{1, \mathrm{G}}=k_{1}^{-} / k_{1}^{+}=0.01 \mathrm{mM} ; \mathrm{K}_{2, \mathrm{G}}=k_{2}^{-} / k_{2}^{+}=0.2 \mathrm{mM} ; \mathrm{k}_{\mathrm{r}, \mathrm{G}}=1.75 \times 10^{-7} \mathrm{~ms}^{-1}} \\
& \mathrm{k}_{\mathrm{e}, \mathrm{G}}=6 \times 10^{-6} \mathrm{~ms}^{-1} ; \xi_{\mathrm{G}}=0.85 ;\left[\mathrm{G}_{\mathrm{T}, \mathrm{G}}\right]=10^{5} ; \mathrm{k}_{\mathrm{deg}, \mathrm{G}}=1.25 \times 10^{-3} \mathrm{~ms}^{-1} ; \mathrm{k}_{\mathrm{a}, \mathrm{G}}=0.17 \times 10^{-3} \mathrm{~ms}^{-1} \\
& \left.\mathrm{k}_{\mathrm{d}, \mathrm{G}}=1.5 \times 10^{-3} \mathrm{~ms}^{-1} ; \mathrm{PIP}_{2, \mathrm{~T}}\right]=5 \times 10^{7} ; \mathrm{r}_{\mathrm{r}, \mathrm{G}}=0.015 \times 10^{-3} \mathrm{~ms}^{-1} ; \mathrm{K}_{\mathrm{c}, \mathrm{G}}=0.4 \times 10^{-3} \mathrm{mM} \\
& \alpha_{\mathrm{G}}=2.781 \times 10^{-8} \mathrm{~ms}^{-1} ; \gamma_{\mathrm{G}}=10^{-15} \mathrm{~N}_{\mathrm{AV}} \operatorname{vol}_{\mathrm{i}} ; \mathrm{k}_{\mathrm{p}, \mathrm{G}}=0 \text {. }
\end{aligned}
$$

\subsubsection{4 sGC activation and cGMP formation}

$$
\begin{aligned}
& \bar{V}_{\mathrm{cGMP}}=\mathrm{V}_{\mathrm{cGMP}, \max } \frac{\mathrm{B} 5_{\mathrm{sGC}}[\mathrm{NO}]+[\mathrm{NO}]^{2}}{\mathrm{~A} 0_{\mathrm{sGC}}+\mathrm{A} 1_{\mathrm{sGC}}[\mathrm{NO}]+[\mathrm{NO}]^{2}} \\
& \tau_{\mathrm{sGC}}=\left\{\begin{array}{lc}
\tau_{\mathrm{sGC}}^{\mathrm{a}} & \text { if }\left(\bar{V}_{\mathrm{cGMP}}-V_{\mathrm{cGMP}}\right) \geq 0 \\
\tau_{\mathrm{sGC}}^{\mathrm{d}} & \text { otherwise }
\end{array}\right. \\
& \frac{\mathrm{d} V_{\mathrm{cGMP}}}{\mathrm{d} t}=\frac{\bar{V}_{\mathrm{cGMP}}-V_{\mathrm{cGMP}}}{\tau_{\mathrm{sGC}}} \\
& \frac{\mathrm{d}[\mathrm{cGMP}]}{\mathrm{d} t}=V_{\mathrm{cGMP}}-\mathrm{k}_{\mathrm{pde}, \mathrm{cGMP}} \frac{[\mathrm{cGMP}]^{2}}{[\mathrm{cGMP}]+\mathrm{K}_{\mathrm{m}, \mathrm{pde}}} \\
& \mathrm{k}_{1, \mathrm{sGC}}=2 \times 10^{3} \mathrm{mM}^{-1} \mathrm{~ms}^{-1} ; \mathrm{k}_{-1, \mathrm{sGC}}=15 \times 10^{-3} \mathrm{~ms}^{-1} ; \mathrm{k}_{2, \mathrm{sGC}}=0.64 \times 10^{-5} \mathrm{~ms}^{-1} \\
& \mathrm{k}_{-2, \mathrm{SGC}}=0.1 \times 10^{-6} \mathrm{~ms}^{-1} ; \mathrm{k}_{3, \mathrm{SGC}}=4.2 \mathrm{mM}^{-1} \mathrm{~ms}^{-1} ; \mathrm{k}_{\mathrm{D}, \mathrm{SGC}}=0.4 \times 10^{-3} \mathrm{~ms}^{-1} ; \mathrm{k}_{\mathrm{D} \tau, \mathrm{SGC}}=0.1 \times 10^{-3} \mathrm{~ms}^{-1} \\
& \mathrm{~B} 5_{\mathrm{sGC}}=\mathrm{k}_{2, \mathrm{sGC}} / \mathrm{k}_{3, \mathrm{sGC}}
\end{aligned}
$$




$$
\begin{gathered}
\mathrm{A} 0_{\mathrm{sGC}}=\left(\left(\mathrm{k}_{-1, \mathrm{sGC}}+\mathrm{k}_{2, \mathrm{sGC}}\right) \mathrm{k}_{\mathrm{D}, \mathrm{sGC}}+\mathrm{k}_{-1, \mathrm{sGC}} \mathrm{k}_{-2, \mathrm{sGC}}\right) /\left(\mathrm{k}_{1, \mathrm{sGC}} \mathrm{k}_{3, \mathrm{sGC}}\right) \\
\mathrm{A}_{\mathrm{sGC}}=\left(\left(\mathrm{k}_{1, \mathrm{sGC}}+\mathrm{k}_{3, \mathrm{sGC}}\right) \mathrm{k}_{\mathrm{D}, \mathrm{sGC}}+\left(\mathrm{k}_{2, \mathrm{sGC}}+\mathrm{k}_{-2, \mathrm{sGC}}\right) \mathrm{k}_{1, \mathrm{sGC}}\right) /\left(\mathrm{k}_{1, \mathrm{sGC}} \mathrm{k}_{3, \mathrm{sGC}}\right) \\
\mathrm{k}_{\mathrm{pde}, \mathrm{cGMP}}=0.0695 \times 10^{-3} \mathrm{~ms}^{-1} ; \tau_{\mathrm{sGC}}^{\mathrm{a}}=0.23 \mathrm{~s} ; \tau_{\mathrm{sGC}}^{\mathrm{d}}=10 \mathrm{~s} ; \mathrm{V}_{\mathrm{cGMP}, \max }=1.26 \times 10^{-7} \mathrm{mM} / \mathrm{ms} ; \\
\mathrm{K}_{\mathrm{m}, \mathrm{pde}}=10^{-3} \mathrm{mM}
\end{gathered}
$$

\subsubsection{Ionic balances}

$$
\begin{gathered}
I_{\mathrm{Catotm}}=I_{\mathrm{SOCCa}}+I_{\mathrm{VOCC}}-2 I_{\mathrm{NCX}}+I_{\mathrm{PMCA}}+I_{\mathrm{CaNSC}} \\
\frac{\mathrm{d}[\mathrm{Ca}]_{\mathrm{i}}}{\mathrm{d} t}=-\frac{I_{\mathrm{Catotm}}+I_{\mathrm{SERCA}}-I_{\mathrm{rel}}-I_{\mathrm{IP} 3}+\sum I_{\mathrm{gj}, \mathrm{Ca}}}{\mathrm{z}_{\mathrm{Ca}} \mathrm{Fvol}_{\mathrm{Ca}}}\left(1+\frac{\left[\overline{\mathrm{S}}_{\mathrm{CM}}\right] \mathrm{K}_{\mathrm{d}}}{\left(\mathrm{K}_{\mathrm{d}}+[\mathrm{Ca}]_{\mathrm{i}}\right)^{2}}+\frac{\left[\overline{\mathrm{B}}_{\mathrm{F}}\right] \mathrm{K}_{\mathrm{dB}}}{\left(\mathrm{K}_{\mathrm{dB}}+[\mathrm{Ca}]_{\mathrm{i}}\right)^{2}}\right)^{-1} \\
I_{\mathrm{Natotm}}=I_{\mathrm{NaKCl}}^{\mathrm{Na}}+I_{\mathrm{SOCNa}}+3 I_{\mathrm{NaK}}+3 I_{\mathrm{NCX}}+I_{\mathrm{NaNSC}} ; \frac{\mathrm{d}[\mathrm{Na}]_{\mathrm{i}}}{\mathrm{d} t}=-\frac{I_{\mathrm{Natotm}}+\sum I_{\mathrm{gj}, \mathrm{Na}}}{\mathrm{Fvol}_{\mathrm{i}}} \\
I_{\mathrm{Ktotm}}=I_{\mathrm{NaKCl}}^{\mathrm{K}}+I_{\mathrm{Kv}}+I_{\mathrm{BKCa}}+I_{\mathrm{KNSC}}+I_{\mathrm{Kleak}}-2 I_{\mathrm{NaK}} ; \frac{\mathrm{d}[\mathrm{K}]_{\mathrm{i}}}{\mathrm{d} t}=-\frac{I_{\mathrm{Ktotm}}+\sum I_{\mathrm{gj}, \mathrm{K}}}{\mathrm{Fvol}_{\mathrm{i}}} \\
I_{\mathrm{Cltotm}}=I_{\mathrm{NaKCl}}^{\mathrm{Cl}}+I_{\mathrm{ClCa}} ; \frac{\mathrm{d}[\mathrm{Cl}]_{\mathrm{i}}}{\mathrm{d} t}=-\frac{I_{\mathrm{Cltotm}}+\sum I_{\mathrm{gj}, \mathrm{Cl}}}{\mathrm{z}_{\mathrm{Cl}} \mathrm{Fvol}_{\mathrm{i}}}
\end{gathered}
$$$$
\left[\overline{\mathrm{S}}_{\mathrm{CM}}\right]=0.1 \mathrm{mM} ;\left[\overline{\mathrm{B}}_{\mathrm{F}}\right]=0.1 \mathrm{mM} ; \mathrm{K}_{\mathrm{d}}=260 \mathrm{nM} ; \mathrm{K}_{\mathrm{dB}}=530 \mathrm{nM} ; \mathrm{vol}_{\mathrm{i}}=1.0 \mathrm{pL} ; \operatorname{vol}_{\mathrm{Ca}}=0.7 \mathrm{pL} .
$$

\subsubsection{Initial conditions}

$V_{\mathrm{m}}=-52.7 \mathrm{mV} ;[\mathrm{Ca}]_{\mathrm{i}}=96 \mathrm{nM} ;[\mathrm{Ca}]_{\mathrm{u}}=0.77 \mathrm{mM} ;[\mathrm{Ca}]_{\mathrm{r}}=0.73 \mathrm{mM} ;[\mathrm{Na}]_{\mathrm{i}}=9.4 \mathrm{mM} ;[\mathrm{K}]_{\mathrm{i}}$

$$
=121 \mathrm{mM} ;[\mathrm{Cl}]_{\mathrm{i}}=42.0 \mathrm{mM} ;\left[\mathrm{IP}_{3}\right]=[\mathrm{DAG}]=[\mathrm{cGMP}]=0 ; V_{\mathrm{cGMP}}=0
$$

$I_{\text {VOCC }}: d_{\mathrm{L}}=1 /\left(1+\exp \left(-V_{\mathrm{m}} / 8.3 \mathrm{mV}\right)\right) ; f_{\mathrm{L}}=1 /\left(1+\exp \left(\left(V_{\mathrm{m}}+42.0 \mathrm{mV}\right) / 9.1 \mathrm{mV}\right)\right)$

$$
\begin{gathered}
I_{\mathrm{BKCa}}: p_{\mathrm{f}}=p_{\mathrm{s}}=1 /\left(1+\exp \left(-\left(V_{\mathrm{m}}-\mathrm{V}_{1 / 2 \mathrm{KCa}}\right) / 18.25 \mathrm{mV}\right)\right) \\
I_{\mathrm{Kv}}: p_{\mathrm{K}}=1 /\left(1+\exp \left(-\left(V_{\mathrm{m}}+11\right) / 15\right)\right) ; q_{1}=q_{2}=1.0 /\left(1+\exp \left(\left(V_{\mathrm{m}}+40\right) / 14\right)\right) \\
\mathrm{RyR}: \mathrm{R}_{01}=0.0012 ; \mathrm{R}_{10}=0.003 ; \mathrm{R}_{11}=3.62 \times 10^{-6} \\
\mathrm{IP}_{3} \mathrm{R}: h_{\mathrm{IP} 3}=\mathrm{K}_{\text {inh,IP3}} /\left([\mathrm{Ca}]_{\mathrm{i}}+\mathrm{K}_{\text {inh, IP3 }}\right)
\end{gathered}
$$


$\alpha 1$-adrenoceptor: $\left[\mathrm{R}_{\mathrm{G}}^{\mathrm{S}}\right]=\left[\mathrm{R}_{\mathrm{T}, \mathrm{G}}\right] \cdot \xi_{\mathrm{G}} ;\left[\mathrm{R}_{\mathrm{P}, \mathrm{G}}^{\mathrm{S}}\right]=0 ;\left[\mathrm{PIP}_{2}\right]=\left[\mathrm{PIP}_{2, \mathrm{~T}}\right]-\left(1+\mathrm{k}_{\mathrm{deg}, \mathrm{G}} / \mathrm{r}_{\mathrm{r}, \mathrm{G}}\right) \gamma_{\mathrm{G}}\left[\mathrm{IP}_{3}\right]$; $r_{\mathrm{h}, \mathrm{G}}=\mathrm{k}_{\mathrm{deg}, \mathrm{G}} \cdot \gamma_{\mathrm{G}}\left[\mathrm{IP}_{3}\right] /\left[\mathrm{PIP} \mathrm{P}_{2}\right] ; \mathrm{G}=r_{\mathrm{h}, \mathrm{G}}\left(\mathrm{K}_{\mathrm{c}, \mathrm{G}}+[\mathrm{Ca}]_{\mathrm{i}}\right) /\left(\alpha_{\mathrm{G}}[\mathrm{Ca}]_{\mathrm{i}}\right) ; \delta_{\mathrm{G}}=\mathrm{k}_{\mathrm{d}, \mathrm{G}} \cdot \mathrm{G} /\left(\mathrm{k}_{\mathrm{a}, \mathrm{G}}\left(\left[\mathrm{G}_{\mathrm{T}, \mathrm{G}}\right]-\right.\right.$ G))

\subsubsection{Endothelial cells}

\subsubsection{Membrane electrophysiology}

$$
\begin{gathered}
\frac{\mathrm{d} V_{\mathrm{m}}}{\mathrm{d} t}=-\frac{1}{\mathrm{C}_{\mathrm{m}}}\left(I_{\mathrm{SOC}}+I_{\mathrm{NSC}}+I_{\mathrm{VRAC}}+I_{\mathrm{CaCC}}+I_{\mathrm{K}_{\mathrm{ir}}}+I_{\mathrm{IK}_{\mathrm{Ca}}}+I_{\mathrm{SK}_{\mathrm{Ca}}}+I_{\mathrm{NaK}}+I_{\mathrm{NCX}}+I_{\mathrm{PMCA}}+\sum I_{\mathrm{gj}}-I_{\mathrm{stim}}\right) \\
\mathrm{C}_{\mathrm{m}}=14 \mathrm{pF} .
\end{gathered}
$$

7.1.3.1.1 Inward rectifier potassium channel

$$
\begin{gathered}
I_{\text {Kir }}=\frac{G_{\text {Kirmax }}\left(V_{\mathrm{m}}-E_{\mathrm{K}}\right)}{1+e^{\frac{\Delta V-\Delta V_{\text {Kirh }}}{v_{\text {Kir }}}}} \\
G_{\text {Kir,max }}=\mathrm{G}_{\text {Kir }}\left(\left[\mathrm{K}^{+}\right]_{0}\right)^{\mathrm{n}_{\text {Kir }}} \\
\Delta V=V_{\mathrm{m}}-E_{\mathrm{K}} \\
\Delta \mathrm{V}_{\text {Kir,h }}=39.42 \mathrm{mV} ; v_{\mathrm{Kir}}=7.084 \mathrm{mV} ; \mathrm{G}_{\mathrm{Kir}}=0.1423 \mathrm{nS} / \mathrm{mM}^{0.5} ; \mathrm{n}_{\text {Kir }}=0.5 .
\end{gathered}
$$

7.1.3.1.2 Calcium-activated potassium channels

$$
\begin{aligned}
& I_{\mathrm{X}}=\mathrm{G}_{\mathrm{X}} P_{\mathrm{O}, \mathrm{X}}\left(V_{\mathrm{m}}-E_{\mathrm{K}}\right) \\
& P_{\mathrm{O}, \mathrm{X}}=\frac{\left[\mathrm{Ca}^{2+}\right]_{\mathrm{i}}^{\mathrm{n}_{\mathrm{X}}}}{\left[\mathrm{Ca}^{2+}\right]_{\mathrm{i}}^{\mathrm{n}_{\mathrm{X}}}+\mathrm{K}_{\mathrm{X}, \mathrm{Cai}}^{\mathrm{n}_{\mathrm{X}}}} \\
& \text { where } \mathrm{X} \text { denotes } \mathrm{SK}_{\mathrm{Ca}} \text { or } \mathrm{IK}_{\mathrm{Ca}} .
\end{aligned}
$$

$\mathrm{G}_{\mathrm{SKCa}}=0.62 \mathrm{nS} ; \mathrm{G}_{\mathrm{IKCa}}=1.72 \mathrm{nS} ; \mathrm{n}_{\mathrm{SKCa}}=5 \mathrm{n}_{\mathrm{IKCa}}=4 ; \mathrm{K}_{\mathrm{SKCa}, \mathrm{Cai}}=237 \mathrm{nM} ; \mathrm{K}_{\mathrm{IKCa}, \mathrm{Cai}}=740$ $\mathrm{nM}$. 
7.1.3.1.3 Calcium-activated chloride channel

$$
\begin{gathered}
I_{\mathrm{CaCC}}=\mathrm{G}_{\mathrm{CaCC}} P_{\mathrm{O}, \mathrm{CaCC}} \frac{1}{1+\left(\frac{\mathrm{K}_{\mathrm{CaCCCai}}}{\left[\mathrm{Ca}^{2+}\right]_{\mathrm{i}}}\right)^{\mathrm{n}_{\mathrm{CaCC}}}}\left(V_{\mathrm{m}}-E_{\mathrm{Cl}}\right) \\
\frac{\mathrm{d} P_{\mathrm{O}, \mathrm{CaCC}}}{\mathrm{d} t}=\frac{P_{\mathrm{O}, \mathrm{CaCCSS}}-P_{\mathrm{O}, \mathrm{CaCC}}}{\tau_{\mathrm{CaCC}}} \\
P_{\mathrm{O}, \mathrm{CaCC}, \mathrm{ss}}=\frac{1}{1+\mathrm{e}^{-\left(V_{\mathrm{m}}-\mathrm{V}_{\mathrm{CaCC}, \mathrm{h}}\right) / v_{\mathrm{CaCC}}}} \\
\tau_{\mathrm{CaCC}}=386.2 e^{-\left(\frac{V_{\mathrm{m}}-19.9 \mathrm{mV} \mathrm{mV}}{88.9 \mathrm{mV}}\right)^{2}} \quad[\mathrm{~ms}]
\end{gathered}
$$

$\mathrm{V}_{\mathrm{CaCC}, \mathrm{h}}=662 \mathrm{mV} ; v_{\mathrm{CaCC}}=132 \mathrm{mV} ; \mathrm{G}_{\mathrm{CaCC}}=37.38 \mathrm{nS} ; \mathrm{n}_{\mathrm{CaCC}}=1.89 ; \mathrm{K}_{\mathrm{CaCC}, \mathrm{Cai}}=287 \mathrm{nM}$.

7.1.3.1.4 Volume-regulated anion channel

$$
\begin{gathered}
I_{\text {VRAC }}=\mathrm{G}_{\text {VRAC }}\left(V_{\mathrm{m}}-E_{\mathrm{Cl}}\right) \\
\mathrm{G}_{\text {VRAC }}=0.381 \mathrm{nS}
\end{gathered}
$$

\subsection{Store-operated cation channel}

$$
\begin{aligned}
& I_{\mathrm{SOC}, \mathrm{Na}}=P_{\mathrm{SOC}, \mathrm{Na}} \mathrm{A}_{\mathrm{m}} \frac{\mathrm{F}^{2}}{\mathrm{RT}} V_{\mathrm{m}} \frac{\left[\mathrm{Na}^{+}\right]_{\mathrm{i}}-\left[\mathrm{Na}^{+}\right]_{0} \mathrm{e}^{\frac{-V_{\mathrm{m}} \mathrm{F}}{\mathrm{RT}}}}{1-\mathrm{e}^{\frac{-V_{\mathrm{m}} \mathrm{F}}{\mathrm{RT}}}} \\
& I_{\text {SOC,Ca }}=\mathrm{P}_{\mathrm{SOC}, \mathrm{Ca}} \mathrm{A}_{\mathrm{m}} \frac{\mathrm{z}_{\mathrm{Ca}}^{2} \mathrm{~F}^{2}}{\mathrm{RT}} V_{\mathrm{m}} \frac{\left[\mathrm{Ca}^{2+}\right]_{\mathrm{i}}-\left[\mathrm{Ca}^{2+}\right]_{\mathrm{o}} \mathrm{e}^{\frac{-\mathrm{zC}_{\mathrm{C}} \gamma_{\mathrm{m}} \mathrm{F}}{\mathrm{RT}}}}{1-\mathrm{e}^{\frac{-\mathrm{z}_{\mathrm{a}} \gamma_{\mathrm{m}} \mathrm{F}}{\mathrm{RT}}}} \\
& I_{\mathrm{SOC}}=P_{\mathrm{O}, \mathrm{SOC}}\left(I_{\mathrm{SOCNa}}+I_{\mathrm{SOC}, \mathrm{Ca}}\right) \\
& P_{\mathrm{SOC}, \mathrm{Na}}=\frac{\mathrm{P}_{\mathrm{SOCNa}, \max }}{1+\left(\frac{\left[\mathrm{Ca}^{2+}\right]_{0}}{\mathrm{~K}_{\mathrm{SOC}, \mathrm{Cao}}}\right)^{\mathrm{n}_{\mathrm{SOC}, \mathrm{Na}}}}
\end{aligned}
$$




$$
P_{O, S O C}=\frac{0.25}{1+\left(\frac{\left[\mathrm{Ca}^{2+}\right]_{\mathrm{IS}}}{\mathrm{K}_{\mathrm{SOC}, \mathrm{CaIS}}}\right)^{n_{S O C}}}+0.083
$$

$$
\begin{gathered}
P_{\mathrm{SOCNa}, \max }=3.95 \times 10^{-7} \mathrm{~cm} / \mathrm{s} ; \mathrm{P}_{\mathrm{SOC}, \mathrm{Ca}}=1.15 \times 10^{-7} \mathrm{~cm} / \mathrm{s} ; \mathrm{n}_{\mathrm{SOC}, \mathrm{Na}}=0.622 ; \mathrm{n}_{\mathrm{SOC}}=3.2 ; \\
\mathrm{K}_{\mathrm{SOC}, \mathrm{CaIS}}=0.47 \mathrm{mM} ; \mathrm{K}_{\mathrm{SOC}, \mathrm{Cao}}=2.0 \times 10^{-4} \mathrm{mM} ; \mathrm{A}_{\mathrm{m}}=14 \times 10^{-6} \mathrm{~cm}^{2}
\end{gathered}
$$

\subsection{Nonselective cation channel}

$$
\begin{aligned}
& I_{\mathrm{NSC}, \mathrm{Na}}=P_{\mathrm{NSC}, \mathrm{Na}} \mathrm{A}_{\mathrm{m}} \frac{\mathrm{F}^{2}}{\mathrm{RT}} V_{\mathrm{m}} \frac{\left[\mathrm{Na}^{+}\right]_{\mathrm{i}}-\left[\mathrm{Na}^{+}\right]_{\mathrm{o}} \mathrm{e}^{\frac{-\mathrm{I}_{\mathrm{m}} \mathrm{F}}{\mathrm{RT}}}}{1-\mathrm{e}^{\frac{-V_{\mathrm{m}} \mathrm{F}}{\mathrm{RT}}}} \\
& I_{\mathrm{NSC}, \mathrm{K}}=\mathrm{P}_{\mathrm{NSC}, \mathrm{K}} \mathrm{A}_{\mathrm{m}} \frac{\mathrm{F}^{2}}{\mathrm{RT}} V_{\mathrm{m}} \frac{\left[\mathrm{K}^{+}\right]_{\mathrm{i}}-\left[\mathrm{K}^{+}\right]_{\mathrm{o}} \mathrm{e}^{\frac{-V_{\mathrm{m}} \mathrm{F}}{\mathrm{RT}}}}{1-\mathrm{e}^{\frac{-V_{\mathrm{m}} \mathrm{F}}{\mathrm{RT}}}} \\
& I_{\mathrm{NSC}, \mathrm{Ca}}=\mathrm{P}_{\mathrm{NSC}, \mathrm{Ca}} \mathrm{A}_{\mathrm{m}} \frac{\mathrm{z}_{\mathrm{Ca}}^{2} \mathrm{~F}^{2}}{\mathrm{RT}} V_{\mathrm{m}} \frac{\left[\mathrm{Ca}^{2+}\right]_{\mathrm{i}}-\left[\mathrm{Ca}^{2+}\right]_{\mathrm{o}} \mathrm{e}^{\frac{-2 \mathrm{ca}_{\mathrm{a}} \mathrm{T}_{\mathrm{m}}^{\mathrm{F}}}{\mathrm{RT}}}}{1-\mathrm{e}^{\frac{-\mathrm{z}_{\mathrm{Ca}} J_{\mathrm{m}}^{\mathrm{F}}}{\mathrm{RT}}}} \\
& I_{\mathrm{NSC}}=I_{\mathrm{NSCNa}}+I_{\mathrm{NSCK}}+I_{\mathrm{NSCCa}} \\
& P_{\mathrm{NSC}, \mathrm{Na}}=\frac{\mathrm{P}_{\mathrm{NSC}, \mathrm{Namax}}}{1+\left(\frac{\left[\mathrm{Ca}^{2+}\right]_{\mathrm{o}}}{\mathrm{K}_{\mathrm{NSC}_{\mathrm{C}} \mathrm{o}}}\right)^{\mathrm{n}_{\mathrm{NSC}}}}
\end{aligned}
$$

$\mathrm{P}_{\mathrm{NSC}, \mathrm{Namax}}=5.34 \times 10^{-8} \mathrm{~cm} / \mathrm{s} ; \mathrm{P}_{\mathrm{NSC}, \mathrm{K}}=0.49 \times 10^{-7} \mathrm{~cm} / \mathrm{s} ; \mathrm{P}_{\mathrm{NSC}, \mathrm{Ca}}=2.4 \times 10^{-8} \mathrm{~cm} / \mathrm{s}$

\subsection{Sodium-calcium exchanger}

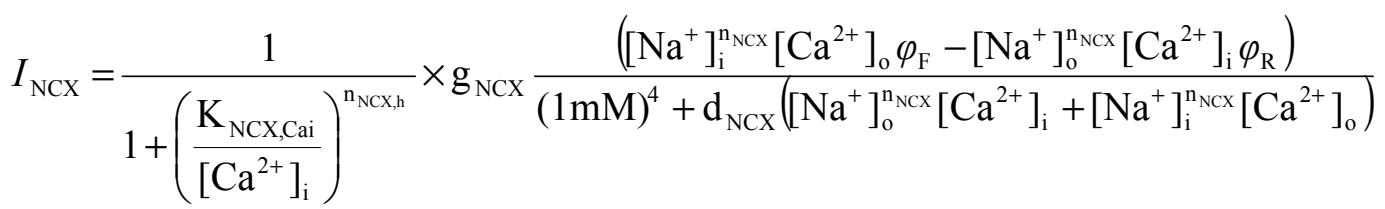

$$
\begin{aligned}
& \varphi_{\mathrm{F}}=\mathrm{e}^{\gamma V_{\mathrm{m}}\left(\mathrm{n}_{\mathrm{NCX}}-2\right) z_{\mathrm{NCX}} \mathrm{F} / \mathrm{RT}} \\
& \varphi_{\mathrm{R}}=\mathrm{e}^{(\gamma-1) V_{\mathrm{m}}\left(\mathrm{n}_{\mathrm{NCX}}-2\right) z_{\mathrm{NCX}} \mathrm{F} / \mathrm{RT}}
\end{aligned}
$$




$$
\begin{gathered}
\mathrm{g}_{\mathrm{NCX}}=1.99 \mathrm{pA} ; \mathrm{n}_{\mathrm{NCX}, \mathrm{h}}=1.50 ; \mathrm{n}_{\mathrm{NCX}}=3 ; \gamma=0.4834 ; \mathrm{d}_{\mathrm{NCX}}=3.04 \times 10^{-4} ; \mathrm{z}_{\mathrm{NCX}}=1 ; \\
\mathrm{K}_{\mathrm{NCX}, \text { Cai }}=0.502 \mathrm{mM} .
\end{gathered}
$$

\subsection{Sodium-potassium $\left(\mathrm{Na}^{+} / \mathrm{K}^{+}\right)$ATPase}

$$
\begin{gathered}
I_{\mathrm{NaK}}=\overline{\mathrm{I}}_{\mathrm{NaK}} \frac{\left[\mathrm{K}^{+}\right]_{\mathrm{o}}}{\left[\mathrm{K}^{+}\right]_{\mathrm{o}}+\mathrm{K}_{\mathrm{NaK}, \mathrm{Ko}}} \times \frac{\left[\mathrm{Na}^{+}\right]_{\mathrm{i}}^{1.5}}{\left[\mathrm{Na}^{+}\right]_{\mathrm{i}}^{1.5}+\mathrm{K}_{\mathrm{NaK}, \mathrm{Nai}}^{1.5}} \times \frac{V_{\mathrm{m}}+135.1 \mathrm{mV}}{V_{\mathrm{m}}+300 \mathrm{mV}} \\
\overline{\mathrm{I}}_{\mathrm{NaK}}=20.18 \mathrm{pA} ; \mathrm{K}_{\mathrm{NaK}, \mathrm{Ko}}=1.32 \mathrm{mM} ; \mathrm{K}_{\mathrm{NaK}, \mathrm{Nai}}=14.52 \mathrm{mM} .
\end{gathered}
$$

\subsection{Sodium-potassium-chloride $\left(\mathrm{Na}^{+} / \mathrm{K}^{+} / 2 \mathrm{Cl}^{-}\right)$cotransport}

$$
\begin{aligned}
I_{\mathrm{NaKCl}_{-} \mathrm{Cl}} & =-L_{\mathrm{NaKCl}} A_{\mathrm{m}} R T F z_{\mathrm{Cl}} \ln \left[\frac{\left[\mathrm{Na}^{+}\right]_{\mathrm{o}}}{\left[\mathrm{Na}^{+}\right]_{\mathrm{i}}} \frac{\left[\mathrm{K}^{+}\right]_{\mathrm{o}}}{\left[\mathrm{K}^{+}\right]_{\mathrm{i}}}\left(\frac{\left[\mathrm{Cl}^{-}\right]_{\mathrm{o}}}{\left[\mathrm{Cl}^{-}\right]_{\mathrm{i}}}\right)^{2}\right] \\
I_{\mathrm{NaKCl} \_\mathrm{K}} & =I_{\mathrm{NaKCl} \_\mathrm{Na}}=-0.5 I_{\mathrm{NaKCl} \_\mathrm{Cl}} \\
\mathrm{L}_{\mathrm{NaKCl}}= & =3.2 \times 10^{-9}(\mathrm{mmol})^{2} \mathrm{~J}^{-1} \mathrm{~s}^{-1} \mathrm{~cm}^{-2} .
\end{aligned}
$$

7.1.3.1.10 Plasma membrane calcium ATPase

$$
\begin{gathered}
I_{\mathrm{PMCA}}=\overline{\mathrm{I}}_{\mathrm{PMCA}} \frac{\left[\mathrm{Ca}^{2+}\right]_{\mathrm{i}}^{\mathrm{n}_{\mathrm{PMCA}}}}{\left[\mathrm{Ca}^{2+}\right]_{\mathrm{i}}^{\mathrm{n}_{\mathrm{P} C C A}}+\mathrm{K}_{\text {PMCA,Cai }}^{\mathrm{n}_{\mathrm{PMCA}}}} \\
\overline{\mathrm{I}}_{\mathrm{PMCA}}=2.67 \mathrm{pA} ; \mathrm{K}_{\mathrm{PMCA}, \mathrm{Cai}}=0.260 \times 10^{-3} \mathrm{mM} ; \mathrm{n}_{\mathrm{PMCA}}=1.4 .
\end{gathered}
$$

Nernst potential

$$
E_{A}=\frac{R T}{z_{\mathrm{A}} F} \ln \left(\frac{[\mathrm{A}]_{\mathrm{o}}}{[\mathrm{A}]_{\mathrm{i}}}\right) \text { where } \mathrm{A} \text { denotes } \mathrm{K}^{+}, \mathrm{Na}^{+}, \mathrm{Ca}^{2+} \text { or } \mathrm{Cl}^{-} \text {. }
$$

\subsubsection{Fluid compartment model}

\subsubsection{1 $\mathrm{IP}_{3}$ receptor}

$$
I_{\mathrm{IP} 3 \mathrm{R}}=\overline{\mathrm{I}}_{\mathrm{IP} 3 \mathrm{R}} \frac{\left[\mathrm{IP}_{3}\right]^{3.8}}{\left[\mathrm{IP}_{3}\right]^{3.8}+\mathrm{K}_{\mathrm{m}, \mathrm{IP} 3}^{3.8}} P_{\mathrm{i}, \mathrm{IP} 3 \mathrm{R}}\left(\left[\mathrm{Ca}^{2+}\right]_{\mathrm{IS}}-\left[\mathrm{Ca}^{2+}\right]_{\mathrm{i}}\right)
$$




$$
\begin{gathered}
P_{\mathrm{i}, \mathrm{IP} 3 \mathrm{R}}=\frac{\mathrm{K}_{\mathrm{i}, \text { Cai }}^{3.8}}{\left[\mathrm{Ca}^{2+}\right]_{i}^{3.8}+\mathrm{K}_{\mathrm{i}, \text { Cai }}^{3.8}} \\
\overline{\mathrm{I}}_{\mathrm{IP} 3 \mathrm{R}}=4.67 \times 10^{6} \mathrm{pA} / \mathrm{mM} ; \mathrm{K}_{\mathrm{m}, \mathrm{IP} 3}=1.6 \times 10^{-3} \mathrm{mM} ; \mathrm{K}_{\mathrm{i}, \mathrm{Cai}}=10^{-3} \mathrm{mM} .
\end{gathered}
$$

7.1.3.2.2 Endoplasmic reticulum calcium ATPase and ER leak

$$
\begin{gathered}
I_{\text {SERCA }, \text { IS }}=\overline{\mathrm{I}}_{\text {SERCA,IS }}\left(\frac{\left[\mathrm{Ca}^{2+}\right]_{\mathrm{i}}}{\left[\mathrm{Ca}^{2+}\right]_{\mathrm{i}}+\mathrm{K}_{\mathrm{SERCA}, \mathrm{IS}}}\right)^{2} \\
I_{\text {leak }, \text { IS }}=\mathrm{k}_{\text {leak }, \text { IS }}\left(\left[\mathrm{Ca}^{2+}\right]_{\mathrm{IS}}-\left[\mathrm{Ca}^{2+}\right]_{\mathrm{i}}\right)^{2} \\
\overline{\mathrm{I}}_{\mathrm{SERCA}, \text { IS }}=0.88 \mathrm{pA} ; \mathrm{K}_{\mathrm{SERCA}, \mathrm{IS}}=0.15 \times 10^{-3} \mathrm{mM} ; \mathrm{k}_{\text {leak }, \mathrm{IS}}=0.0176 \mathrm{pA} /(\mathrm{mM})^{2} .
\end{gathered}
$$

\subsubsection{Intracellular ionic and material balances}

$$
\begin{aligned}
& I_{\mathrm{Catotm}}=I_{\mathrm{SOC}, \mathrm{Ca}}-2 I_{\mathrm{NCX}}+I_{\mathrm{PMCA}}+I_{\mathrm{NSC}, \mathrm{Ca}} \\
& \frac{\mathrm{d}\left[\mathrm{Ca}^{2+}\right]_{\mathrm{i}}}{\mathrm{d} t}=-\frac{I_{\text {Catotm }}+I_{\text {SERCA }, \text { IS }}-I_{\text {leak }, \text { IS }}-I_{\text {IP3R }}+\sum I_{\mathrm{g}, \mathrm{Ca}}}{\mathrm{z}_{\mathrm{Ca}} \mathrm{Fvol}_{\mathrm{Ca}}}-\frac{\mathrm{d}\left[\mathrm{Ca}^{2+}\right]_{\mathrm{B}}}{\mathrm{d} t} \\
& \frac{\mathrm{d}\left[\mathrm{Ca}^{2+}\right]_{\mathrm{B}}}{\mathrm{d} t}=\mathrm{kB}_{\text {on }}\left[\mathrm{Ca}^{2+}\right]_{\mathrm{i}}\left(\mathrm{B}_{\mathrm{T}}-\left[\mathrm{Ca}^{2+}\right]_{\mathrm{B}}\right)-\mathrm{kB}_{\text {off }}\left[\mathrm{Ca}^{2+}\right]_{\mathrm{B}} \\
& \frac{\mathrm{d}\left[\mathrm{Ca}^{2+}\right]_{\mathrm{IS}}}{\mathrm{d} t}=-\frac{I_{\mathrm{IP} 3 \mathrm{R}}-I_{\mathrm{SERCA}, \mathrm{IS}}+I_{\text {leak }, \mathrm{IS}}}{\mathrm{z}_{\mathrm{Ca}} \mathrm{FvOl}_{\mathrm{IS}}}\left[1+\frac{\overline{\mathrm{CSQN}} \cdot \mathrm{K}_{\mathrm{CSQN}}}{\left(\mathrm{K}_{\mathrm{CSQN}}+\left[\mathrm{Ca}^{2+}\right]_{\mathrm{IS}}\right)^{2}}\right]^{-1} \\
& \frac{\mathrm{d}\left[\mathrm{Na}^{+}\right]_{\mathrm{i}}}{\mathrm{d} t}=-\frac{I_{\mathrm{SOC}, \mathrm{Na}}+3 I_{\mathrm{NCX}}+I_{\mathrm{NSC}, \mathrm{Na}}+3 I_{\mathrm{NaK}}+I_{\mathrm{NaKCl} \_\mathrm{Na}}+\sum I_{\mathrm{gj}, \mathrm{Na}}}{\mathrm{z}_{\mathrm{Na}} \mathrm{Fvol}_{\mathrm{i}}} \\
& \frac{\mathrm{d}\left[\mathrm{K}^{+}\right]_{\mathrm{i}}}{\mathrm{d} t}=-\frac{I_{\mathrm{SK}_{\mathrm{Ca}}}+I_{\mathrm{IK}_{\mathrm{Ca}}}+I_{\mathrm{Kir}}+I_{\mathrm{NSC}, \mathrm{K}}-2 I_{\mathrm{NaK}}+I_{\mathrm{NaKCl} \_\mathrm{K}}+\sum I_{\mathrm{gj}, \mathrm{K}}}{\mathrm{Z}_{\mathrm{K}} \mathrm{Fvol}_{\mathrm{i}}} \\
& \frac{\mathrm{d}\left[\mathrm{C}^{\digamma}\right]_{\mathrm{i}}}{\mathrm{d} t}=-\frac{I_{\mathrm{NaKCl} \_\mathrm{Cl}}+I_{\mathrm{VRAC}}+I_{\mathrm{CaCC}}+\sum I_{\mathrm{g}, \mathrm{Cl}}}{\mathrm{z}_{\mathrm{Cl}} \mathrm{Fvol}} \\
& \frac{\mathrm{d}\left[\mathrm{IP}_{3}\right]}{\mathrm{d} t}=Q_{\mathrm{GIP} 3}-\mathrm{k}_{\mathrm{DIP} 3}\left[\mathrm{IP}_{3}\right]+\frac{\sum J_{\mathrm{IP} 3}}{\mathrm{vol}_{\mathrm{i}}} \\
& \frac{\mathrm{d} Q_{\mathrm{GIP} 3}}{\mathrm{~d} t}=\frac{\mathrm{Q}_{\mathrm{GIP} 3 \$ \mathrm{~S}}-Q_{\mathrm{GIP} 3}}{\tau_{\mathrm{IP}}}
\end{aligned}
$$




$$
\begin{gathered}
\mathrm{kB}_{\text {on }}=100(\mathrm{mM})^{-1 .}(\mathrm{ms})^{-1} ; \mathrm{kB}_{\text {off }}=0.300(\mathrm{~ms})^{-1} ; \mathrm{B}_{\mathrm{T}}=0.120 \mathrm{mM} ; \overline{\mathrm{CSQN}}=15 \mathrm{mM} ; \mathrm{K}_{\mathrm{CSQN}} \\
=0.8 \mathrm{mM} ; \mathrm{Q}_{\mathrm{GIP} 3, \mathrm{SS}}=5.5 \times 10^{-8} \mathrm{mM} / \mathrm{ms} ; \mathrm{k}_{\mathrm{DIP} 3}=2.0 \times 10^{-3}(\mathrm{~ms})^{-1} ; \tau_{I P_{3}}=5 \mathrm{~s} ; \mathrm{vol}_{\mathrm{i}}=1.173 \mathrm{pL} \\
\operatorname{vol}_{\mathrm{Ca}}=0.912 \mathrm{pL} ; \mathrm{vol}_{\mathrm{IS}}=0.335 \mathrm{pL} .
\end{gathered}
$$

\subsubsection{Initial conditions}

$$
\begin{gathered}
V_{\mathrm{m}}=-49.8 \mathrm{mV} ;\left[\mathrm{Ca}^{2+}\right]_{\mathrm{i}}=131 \mathrm{nM} ;\left[\mathrm{Ca}^{2+}\right]_{\mathrm{IS}}=3.3 \mathrm{mM} ;\left[\mathrm{Na}^{+}\right]_{\mathrm{i}}=18.7 \mathrm{mM} ;\left[\mathrm{K}^{+}\right]_{\mathrm{i}}=116 \\
\mathrm{mM} ;\left[\mathrm{Cl}^{-}\right]_{\mathrm{i}}=46.3 \mathrm{mM} ; \\
P_{\mathrm{O}, \mathrm{CaCC}}=\frac{1}{1+\mathrm{e}^{-\left(V_{\mathrm{m}}-\mathrm{V}_{\mathrm{CaCC}, \mathrm{h}}\right) / v_{\mathrm{CaCC}}}} \\
{\left[\mathrm{Ca}^{2+}\right]_{\mathrm{b}}=\mathrm{kB}_{\mathrm{on}}\left[\mathrm{Ca}^{2+}\right]_{\mathrm{i}} /\left(\mathrm{kB}_{\mathrm{on}}\left[\mathrm{Ca}^{2+}\right]_{\mathrm{i}}+\mathrm{kB}_{\mathrm{off}}\right) \mathrm{B}_{\mathrm{T}} .}
\end{gathered}
$$

\subsection{Model Innovations}

We developed detailed mathematical models describing $\mathrm{Ca}^{2+}$ and $\mathrm{V}_{\mathrm{m}}$ dynamics in vascular SMCs and ECs based on data mostly from rat mesenteric arterioles (RMAs). The models incorporated the most significant membrane currents identified in the particular cell types. Moreover, the models took into account effect of microdomains sturctures (such as microprojections), the uneven distribution of membrane and store components, preferential localization of cellular structures in the microdomains (such high density distribution of $\mathrm{IK}_{\mathrm{Ca}}$, TRPV4s, $\mathrm{IP}_{3} \mathrm{Rs}$ in the miroprojections) and the resulting spatial concentration gradients for ionic species and second messengers. An integrative model approach undertaken here accounts for the complex interactions at subcellular levels to reprodue macroscale responses. Similarly to other modeling approaches, our methodology uses typical Hodgkin-Huxley formalism at the base of the cellular models. The methodology presented here has several novel features including: a) Models that can examine of $\mathrm{Ca}^{2+}$ signaling spanning from subcllular to inter-cellular $\mathrm{Ca}^{2+}$ events; b) cell- 
coupling formulations to monitor ionic and second messengers exchange and the weak intracellular currents; c) integration of signaling pathways (such as NO signaling from EC to SMC); d) Models incoporating accurate microdomain geometries from electron microsocpy images.

\subsection{Model Validation and Sensitivity Analysis}

The models integrate membrane electrophysiology with dynamics for the major ionic species $\left(\mathrm{K}^{+}, \mathrm{Na}^{+} \mathrm{Cl}^{-}\right)$and second messengers $\left(\mathrm{Ca}^{2+}, \mathrm{IP}_{3}, \mathrm{NO}\right)$. We incorporated major components of membrane electrophysiology in typical resistance arterioles. Models include kinetic descriptions for channels, pumps, membrane and store receptors such as non-selective cation (NSC), transient receptor vanilloid 4 (TRPV4), store-operated cation (SOC), small $\left(\mathrm{SK}_{\mathrm{Ca}}\right)$, intermediate $\left(\mathrm{IK}_{\mathrm{Ca}}\right)$ and large $\left(\mathrm{BK}_{\mathrm{Ca}}\right)$ conductance calciumactivated $\mathrm{K}^{+}$, inward-rectifier $\mathrm{K}^{+}(\mathrm{KIR})$, volume-regulated anion (VRAC) and calciumactivated $\mathrm{Cl}^{-}(\mathrm{ClCa})$ channels, $\mathrm{Na}^{+}-\mathrm{Ca}^{2+}$ exchanger (NCX) and $\mathrm{Na}^{+} / \mathrm{K}^{+}$-ATPase pump (NaK), $\mathrm{IP}_{3}\left(\mathrm{IP}_{3} \mathrm{R}\right)$ and ryanodine (RyR) receptors, SERCA and PMCA pumps. The EC model incorporates a $\mathrm{Ca}^{2+}$-dependent $\mathrm{NO}$ release in response to agonist (i.e.

Acetylcholine; ACh) stimulation. Compartmental EC model contains 12 Ordinary Differential Equations (ODEs) and approximately 60 parameters. In the SMC model, a description for agonist activation of $\alpha 1$-adrenoceptor leading to $\mathrm{IP}_{3}$ and $\mathrm{DAG}$ formation is incorporated to simulate the effect of (Norepinephrine; NE). The vasodilatory action of $\mathrm{NO}$ is simulated through an effect on four main targets (i.e. $\mathrm{BK}_{\mathrm{Ca}}, \mathrm{NCX}, \mathrm{Cl}_{\mathrm{Ca}}, \mathrm{NaKCl}$ cotransporter). The model contains 24 ODEs and approximately 100 parameters, the majority of which are estimated from independent experimental studies. Uncertainty in parameter values exists and this poses a significant limitation for the model. Sensitivity 
analysis using the method of Latin Hypercube Sampling is utilized to address this limitation. Most importantly, only a relatively small number of unknown parameters is optimized to fit a variety of integrated responses. Each cellular model is then compared with documented features of cell behavior for validation (i.e. resting concentrations, resting $\mathrm{V}_{\mathrm{m}}, \mathrm{Ca}^{2+}$ and $\mathrm{V}_{\mathrm{m}}$ transients in response to agonist stimulation etc). 
VITA

\section{JAIMIT PARIKH}

Born, Ahmedabad, India

2004-2008

B.E., Biomedical Engineering

L.D. College of Engineering

Ahmedabad, India

2009-2015 Doctoral Candidate

Department of Biomedical Engineering

Florida International University

Miami, Florida, USA

\section{PUBLICATIONS AND PRESENTATIONS}

J.Parikh, A.Kapela and N.M.Tsoukias (2015). "Stochastic model of a TRPV4 Calcium sparklet: Effect of bursting and cooperativity on endothelial-derived hyperpolarization" (In press), Biophys J.

A.Kapela, J.Parikh and N.M.Tsoukias. (2012). "Multiple factors influence calcium synchronization in arterial vasomotion.” Biophys J 102(2): Pg. 211-220.

A.Kapela, S.Nagraja, J.Parikh and N.M.Tsoukias. (2011). "Modeling $\mathrm{Ca}^{2+}$ signaling in the micro-circulation: intercellular communication and vasoreactivity." Crit Rev Biomed Eng 39(5): Pg. 435-460.

J.Parikh, A.Kapela and N.M.Tsoukias. "Localized endothelial nitric oxide-mediated smooth muscle cell hyperpolarization; regulation by endothelial Hemoglobin $\alpha$ and erythrocyte's." (Manuscript under final modifications)

J.Parikh, A.Kapela and N.M.Tsoukias. "Oscillatory calcium waves in vascular cells: wave velocity dependence on membrane electrophysiology." (Manuscript under preparation)

J.Parikh, A.Kapela and N.M.Tsoukias. "Regulation of vascular tone via localized calcium signaling in myoendothelial projections", Pg.1, BMES, October 22-25, San Antonio, Texas, 2014.

J.Parikh, A.Kapela and N.M.Tsoukias. "Calcium oscillations and waves in vascular cells: Mechanisms of initiation and modulation", Pg. 1, BMES, October 22-25, San Antonio, Texas, 2014.

J.Parikh, A.Kapela and N.M.Tsoukias. "Finite element modeling of vasoreactivity using COMSOL Multiphysics® Software”,Pg. 1-3, COMSOL CONFERENCE, October 8-10, Boston, MA, 2014 
J.Parikh, A.Kapela and N.M.Tsoukias. "Localized calcium events and endothelium derived signals in the modulation of vessel tone", Pg.1, ISRA, September 7-11, Banff, Ab, Canada 2014.

J.Parikh, A.Kapela and N.M.Tsoukias. "Endothelial hemoglobin-alpha modulating NO mediated feedback response during smooth muscle stimulation", Pg.1, ISRA, September 7-11, Banff, Ab, Canada 2014.

J.Parikh, A.Kapela and N.M.Tsoukias. "Multi-scale modeling of microvascular reactivity”, Pg.1-2, ICCABS, June 3, Session 6A, Miami, Fl, 2014.

J.Parikh, A. Kapela and N.M. Tsoukias. "Modeling localized calcium signals in vascular cells", Pg.1, NAVBO, October 22, Boston, MA, 2013.

N.M.Tsoukias, A. Kapela and J. Parikh. "Mechanisms regulating localized and global Ca2+ responses in vascular cells", Pg.1, 37th IUPS, July 22, Birmingham, UK, 2013.

A.Kapela, J.Parikh and N.M.Tsoukias. "Computational modeling of vascular reactivity", Pg.1, SBEC, May 5, 158, Miami, FL, 2013.

J.Parikh, A.Kapela and N.M.Tsoukias. "Role of localized calcium events in the regulation of vascular tone: A theoretical investigation", Pg.1, FASEB J, April 20, 27:678.9, Boston, MA, 2013

A.Kapela, J.Parikh and N.M.Tsoukias. "Multiscale computational models of microvascular reactivity from ion channels to intercellular signaling", Pg.1, FASEB J, April 21, 27:901.8, Boston, MA, 2013.

J.Parikh, A.Kapela and N.M. Tsoukias. "Mathematical modeling of intracellular calcium waves: Role of intracellular diffusion and heterogeneity",Pg.1, BMES, October 24 - 27, Atlanta, GA, 2012.

J. Parikh, S. Nagraja, A. Kapela and N.M.Tsoukias. "Role of Intra- and Inter- cellular ionic and second messenger fluxes: A theoretical investigation", Pg.1, 62nd BMS, July 4 - 6, London, UK, 2012.

A.Kapela, J.Parikh, S.Nagraja and N.M.Tsoukias. "Theoretical modeling of Intra- and Inter- cellular spatiotemporal calcium patterns in vascular endothelial and smooth muscle cells”, Pg.1, BMES, October 13, PS-Thurs-A-47, Hartford, CT, 2011.

J. Parikh, A. Kapela, and N.M. Tsoukias. "Role of electrical current and diffusion of second messen-gers in $\mathrm{Ca} 2+$ synchronization during vasomotion in microcirculation: Theoretical models", Pg.1, FASEB J, March 17, 25:816.14, Washington DC, 2011. 